\title{
Diffraction of Metastable Rare-Gas Atoms from Nanostructured Transmission Gratings
}

\author{
Dissertation \\ zur Erlangung des Doktorgrades \\ der Mathematisch-Naturwissenschaftlichen Fakultäten \\ der Georg-August-Universität zu Göttingen
}

vorgelegt von

Christian Peter Walter

aus Neumarkt

Göttingen 2002 
D 7

Referent: Herr Prof. Dr. G. C. Hegerfeldt

Korreferent: Herr Prof. Dr. K. Schönhammer

Tag der mündlichen Prüfung: 27. November 2002 


\section{Contents}

1. Introduction 1

2 Scattering Theory 4

2.1 Experiment . . . . . . . . . . . . . . . . . . . . . . . . 4

2.2 Stationary Scattering Theory . . . . . . . . . . . . . . 5

2.3 Grating Potential . . . . . . . . . . . . . . . . . . . . . . 9

2.4 Diffraction from the Repulsive Potential . . . . . . . . . . . . . . 12

2.5 Eikonal Approximation . . . . . . . . . . . . . . . . . . . . . . 14

2.6 Fresnel Corrections . . . . . . . . . . . . . . . . . . . . . . . . 21

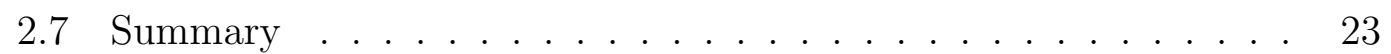

3 van der Waals Potentials 24

3.1 The Dispersion Force . . . . . . . . . . . . . . . . . . . . . . . . . 26

32 Atom-Solid lnteraction . . . . . . . . . . . . . . . . . 31

3.3 Atomic Polarizability . . . . . . . . . . . . . . . . . . . . . . . . . 33

3.4 Response of the Solid . . . . . . . . . . . . . . . . . . . 36

B.5 Discussion . . . . . . . . . . . . . . . . . . . . . . . . . . . . . . . 39

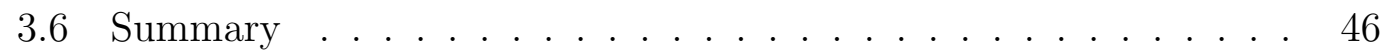

4 Inclusion of Atom-Surface Potentials 48

4.1 Phase Shift . . . . . . . . . . . . . . . . . . . . . . . 48

4.2 Inclined 1 ncidence . . . . . . . . . . . . . . . . 60

$4.2 .1 \quad$ Bar Potential for Rotated Grating . . . . . . . . . . . . . . 60

4.2 .2 Slit Amplitude for Rotated Grating . . . . . . . . . . . . . 63

4.3 Surface Roughness $\ldots \ldots \ldots 7$ 
4.4 Retardation . . . . . . . . . . . . . . . . . . . . . 74

4.5 Adsorbate . . . . . . . . . . . . . . . . . . . . . . . . . . . 79

4.6 Summary $\ldots \ldots \ldots \ldots$. . . . . . . . . . . . . . . . . . . . . . . . . 82

5 Diffraction of Metastable Atoms 85

5.1 The Transmission Grating . . . . . . . . . . . . . . . . . . . . 87

5.1 .1 Period . . . . . . . . . . . . . . . . . . . 88

5.1 .2 Shape of the Grating Bars . . . . . . . . . . . . . . . . . 89

5.1 .3 Slit Width . . . . . . . . . . . . . . . . . . . . . . . . . . 94

5.2 Repulsive Potential . . . . . . . . . . . . . . . . . . . . . . . 109

5.3 Error Analysis . . . . . . . . . . . . . . . . . . . . . . . . . . . . . 118

5.4 Diffraction Experiment with Metastable Atoms . . . . . . . . . . 123

5.4 .1 Beam . . . . . . . . . . . . . . . . . . 123

5.4 .2 Collimation and Diffraction . . . . . . . . . . . . . 124

5.4 .3 Error Sources in the Apparatus . . . . . . . . . . . . . . . 129

5.4 .4 Dispersion Interaction Strength . . . . . . . . . . . . . . . 133

5.5 Summary $\ldots \ldots \ldots \ldots \ldots$. . . . . . . . . . . . . . . 140

6 Summary and Outlook 142

6.1 Summary . . . . . . . . . . . . . . . . . . . . . . . . . . . 142

6.2 Outloot . . . . . . . . . . . . . . . . . . . . . . 144

\begin{tabular}{lr}
\hline A Infinite Half-Space & 146
\end{tabular}

B Grating Bar Potential 148

$\begin{array}{ll}\text { C Retarded Bar Potential } & 150\end{array}$ 


\section{Chapter 1}

\section{Introduction}

In a recent issue the International Herald Tribune dedicated a front page article to the world-wide growing industrial fabrication of products which are structured on the scale of nanometers, for example, computer chips, nano-machinery for medical applications, materials designed for special (aeronautical, automotive) applications, molecular engineering, biotechnology, and so forth [T]. The author points to the technological and sociological importance and potential dangers of this new development taking place only a few decades after microelectronics have found their way into everyday life. As a crucial issue he observes that by going down to such minute scales the -in his view- obscure laws of quantum mechanics are about to play an important role in the realm of industrial engineering which so far has been comfortably ruled by Newton's and Maxwell's intuitive theories. So it seems, with no irony intended, that a century after Max Planck introduced the quantization of action the public finally gets in touch with quantum mechanics. In a way, one might think this right on schedule considering that today global positioning devices are being sold in food discounter stores to large groups of non-professional users. Not all of the buyers are likely to know that the global positioning system can only work with due account of Albert Einstein's special theory of relativity which like Planck's discovery is a child of the early 20th century.

An example of modern nanotechnology are the high-quality silicon nitride transmission gratings made by T. Savas and co-workers at the Massachusetts Institute of Technology (MIT, USA) [2] with a period of $100 \mathrm{~nm}$ and a depth of about the same size. The product is continuously being improved to meet industrial demand for applications such as optical elements in X-ray satellites. Typical wavelengths of X-rays are roughly equal to the de Broglie wavelength of atoms in conventional molecular beams so the transmission gratings have been found suitable for diffraction experiments with atoms which since 1990 has led to the construction of interferometers for atoms by various groups [3, 4]. Other spectacular achievements in this field have been made by demonstrating that large molecules 
like fullerenes [5] and also metastable atoms [6] can be diffracted from nanostructured transmission gratings. Particularly fruitful applications have been realized during the last years by the Toennies group at the Max-Planck-Institut für Strömungforschung, Göttingen, who used the diffraction of a molecular helium beam from a transmission grating to separate He clusters according to their size [7], or focused an atomic beam by means of a nanostructured Fresnel zone plate [8]. With theoretical support from the Hegerfeldt group at the Institut für theoretische Physik der Universität Göttingen the size of the large and fragile helium dimer has been accurately measured for the first time [9] with further implications for other molecules [10, II], and the strength of the van der Waals interaction between rare-gas atoms and the grating material has been determined by diffracting atomic beams from transmission gratings [12]. The latter method bears a high potential of accuracy as the van der Waals interaction strength directly influences the intensity of the principal diffraction order peaks which can be measured very precisely due to the high flux of particles through the grating. The influence of the van der Waals force is also very pronounced because all the narrow grating slits offer a large interacting surface to the passing atoms, which is a definite improvement with respect to similar experiments using a single edge to deflect the atomic beam [13]. Furthermore, the small width of the grating slits allows for definite statements on the influence of retardation effects which greatly facilitates the classification of the measured interaction strength which otherwise has to be interpreted in terms of an atom-surface potential that lies somewhere in between the retarded and the non-retarded limits.

As the measured interaction strengths of rare-gas atoms with the silicon nitride grating material have not yet been quantitatively compared with theoretical values a central aim of this work is to provide these theoretical values and make such a comparison. It will turn out that there are significant discrepancies between the theoretical and the measured values so that a careful review and substantial improvements of the method are presented in this work which finally yield an explanation of the observed deviations. Another systematic discrepancy between theoretical and measured diffraction intensities that has long obscured an adequate interpretation of the measurements is clarified in this work to the benefit of current and future projects that are based on the atom diffraction technique. The comprehensive insight into the method is then used to determine for the first time accurate values of the van der Waals interaction strength of metastable $\mathrm{He}^{*}$ and $\mathrm{Ne}^{*}$ atoms from a new series of experiments. These atoms are of great practical relevance as they are frequently used for diffraction or interferometric experiments [14, 15, 16] some of which are quite similar to those discussed in this work. Especially slow metastable beams have been employed in sophisticated applications like atom holography [17, 18, 1.9] or the recent spectacular realization of Bose-Einstein condensates of metastable $\mathrm{He}^{*}$ [20, 20]. A further step in this direction is to conduct Bose-Einstein condensates in waveg- 
uides or study the collective effects upon interaction with surfaces [22, [23]. An obvious prerequisite for these endeavours is the knowledge of the interaction of single metastable atoms with a solid surface which this work aims to provide both from the theoretical and the experimental side. Additionally, this work is devoted to looking for extensions to the versatility of the accurate atom-optical technique, for example, to answering the question if it is possible to measure the entire atom-surface potential rather than only the long-ranged attractive part. Another important extension is to look for ways to measure the interaction of an atomic beam with surface materials different from the silicon nitride of which the gratings are made at present.

To address the issues raised above this work is organized as follows. Chapter 2 explains the scattering theoretical foundations of atom diffraction from transmission gratings according to the works of G. C. Hegerfeldt and T. Köhler [24, 25, 26]. Use is made of certain principles of scattering and diffraction theory which can be found in textbooks [27, 28, 29, 30, 31, 32, 331, 34]. As a new aspect, the relevance of Fresnel corrections to the previously applied Fraunhofer diffraction limit is investigated for typical experiments, and the consequences of the due inclusion of the Fresnel corrections are worked out.

The following chapter 3 is dedicated to the theory of van der Waals (vdW) forces a complete picture of which is given with the help of literary sources on the quantum electrodynamical origin of the vdW forces, the relevance of atomic and solid state properties, and adequate ways to evaluate the interaction strength of arbitrary materials from optical data. The theoretical values of the interaction strengths of ground-state rare-gas atoms with silicon nitride are then calculated and compared to extant measurements [1:2].

Chapter 4 demonstrates the application of the scattering theoretical results of chapter 2 to the experiments as carried out by the Toennies group. A refined theoretical treatment is shown to open the way to new applications for this type of experiment. The new aspects also help to deliver a comprehensive discussion of the experiments and their interpretation which stands at the beginning of chapter 5. Two important systematical corrections to the technique are motivated as a result of the discussion, furthermore, a new method is presented that allows to determine the total atom surface potential which consists of a short-range repulsive and a long-range attractive part. Finally, the vdW interaction strengths between metastable atoms and the silicon nitride grating material are determined from measured diffraction intensities. In order to increase the significance of the results, various methods of measurement and analysis are compared with each other and with the theoretical values calculated in chapter 3. A detailed treatment of the errors aims to provide a reliable base for current and future experiments. In a final chapter the summaries that conclude each chapter are reviewed and analyzed in view of future projects which are presented in an outlook at the end of this work. 


\section{Chapter 2}

\section{Scattering Theory}

\section{$2.1 \quad$ Experiment}

The following theoretical description is aimed at an experimental situation which is schematically drawn in Fig. 2.1. There is a high-pressure source from which a

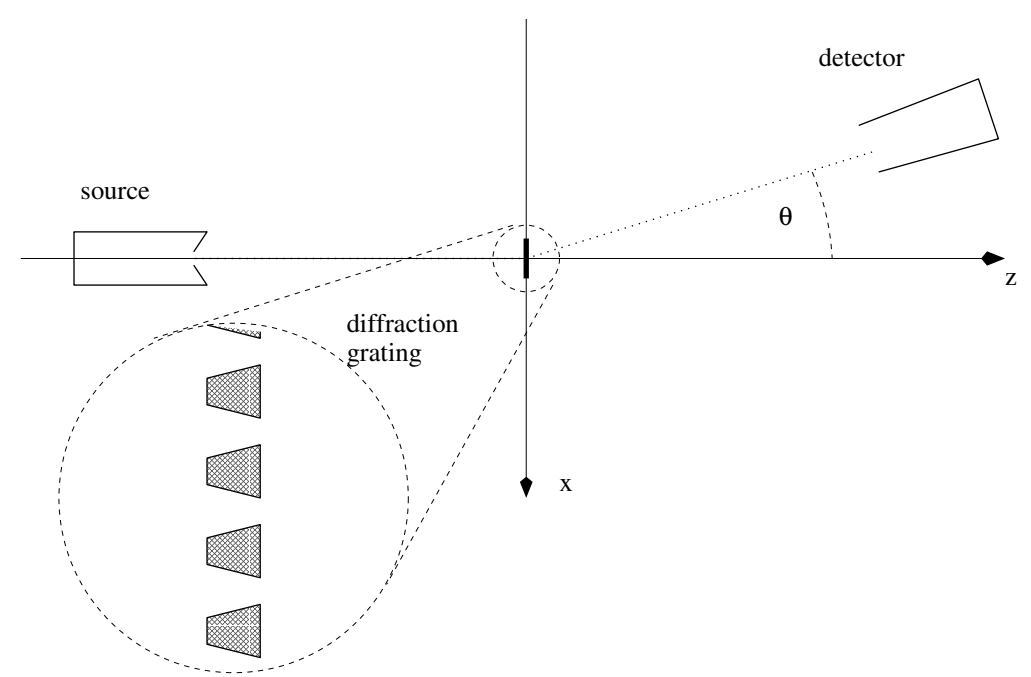

Figure 2.1: Schematic top-view on a typical experiment to be described. The atom source and the detector are each about $1 \mathrm{~m}$ away from the $100 \mathrm{~nm}$-period transmission grating which is illuminated over a width of $10 \mu \mathrm{m}$. The depth of the grating is approximately $100 \mathrm{~nm}$.

spray cloud of independently propagating rare-gas atoms expands into the vacuum inside the apparatus at a typical velocity of $1000 \mathrm{~m} / \mathrm{s}$. By means of collimation slits a $5 \mathrm{~mm}$ high and $10 \mu \mathrm{m}$ wide beam is selected in the forward $z$ direction. 
About $1 \mathrm{~m}$ downstream from the source, at $z=0$ the collimated beam illuminates approximately 100 slits of a $100 \mathrm{~nm}$-period transmission grating that has been designed to maintain its regular structure over the total width and also the total height of the beam [2]. Especially the far-reaching translational invariance of the grating along the (vertical) $y$ direction allows the set-up to be described in a two-dimensional formalism. Approximately $1 \mathrm{~m}$ behind the grating a detecting device records the number of atoms that are scattered at angles $|\vartheta|<10 \mathrm{mrad}$.

The forces between the atoms and the grating are essentially the strong Pauli repulsion of the outer electrons of the atom and the surface at distances of a few Bohr radii, and the van der Waals attraction due to mutually induced charge fluctuations, which extends to distances of a few nanometers. Both forces tend to zero faster than $1 / l^{2}$ for large atom-surface separations $l$, therefore the total scattering potential is short-ranged and can be assumed to vanish near the source and near the detector.

In this work, the interaction of the atomic beam with the transmission grating is assumed to be essentially due to elastic scattering processes. It turns out that for each particle the possibility of directly hitting the surface of the grating bars does not significantly alter the possibility of being transmitted and diffracted by the grating. The possibility of a particle exciting a lattice vibration inside the amorphous silicon nitride compound of the grating bars while passing them is considered negligible.

After all, the scattering potential bears no explicit time dependence so that the theoretical foundations can be conveniently set out in the framework of stationary scattering theory [27, 28, 2.9] .

\subsection{Stationary Scattering Theory}

For an atom of mass $m$, let $|\Psi(t)\rangle$ be the general solution of the scattering problem as described in the previous section, in a sense that it fulfils the timedependent Schrödinger equation

$$
\mathrm{i} \hbar|\dot{\Psi}(t)\rangle=H|\Psi(t)\rangle .
$$

The Hamiltonian $H$ of the system is given by

$$
H=\frac{P^{2}}{2 m}+V(\mathbf{X})=: H_{0}+V(\mathbf{X}),
$$

where $\mathbf{P}$ and $\mathbf{X}$ are the momentum and position operators, respectively. An atom just leaving the source does not feel the short-ranged scattering potential $V$ and is represented by a freely propagating wave packet

$$
\langle\mathbf{r} \mid \Psi(t)\rangle \equiv \Psi(\mathbf{r}, t) \stackrel{z \ll 0}{\longrightarrow} \frac{1}{2 \pi} \int \mathrm{d}^{2} k^{\prime} \breve{\psi}\left(\mathbf{k}^{\prime}\right) \mathrm{e}^{\mathrm{i}\left[\mathbf{k}^{\prime} \mathbf{r}-\omega\left(k^{\prime}\right) t\right]},
$$


with the dispersion relation

$$
\omega\left(k^{\prime}\right)=\frac{\hbar\left(k^{\prime}\right)^{2}}{2 m} .
$$

The symbols $\stackrel{z \ll 0}{\longrightarrow}, z \approx 0$, and $\stackrel{0 \ll z}{\longrightarrow}$ indicate locations near the source, the transmission grating, and the detector, respectively. In this chapter, all coordinate space-related mathematical objects are by default two-dimensional according to Fig. 2.1. In particular, bold type letters denote two-dimensional vectors, e.g. $\mathbf{r} \equiv(x, z)^{\mathrm{T}}$, where the superscript appears to indicate that $\mathbf{r}$ is a column vector. A tilde distinguishes any reference to three dimensions, e.g. $\tilde{\mathbf{r}} \equiv(x, y, z)^{\mathrm{T}}$, or $\tilde{\psi}: \mathbb{R}^{3} \rightarrow \mathbb{C}, \tilde{\mathbf{r}} \mapsto \tilde{\psi}(\tilde{\mathbf{r}})$.

In the present type of experiment the atomic wave packets spend about a millisecond travelling from the source to the detector. Within this time-span their broadening can be neglected and the atoms are thought of as wave packets of constant shape moving along at the group velocity $v=\left.\frac{\mathrm{d} \omega\left(k^{\prime}\right)}{\mathrm{d} k^{\prime}}\right|_{k^{\prime}=k}$ taken at the center $\mathbf{k}$ of the wave packet in momentum space. The well-known concept of the scattering of wave packets (see, e.g. [28]) can thus be applied to the problem. In view of the applications to be made later in this text it is sufficient to neglect even the width of the wave packet in momentum space, which means that all measured quantities can be interpreted in terms of a single sharp velocity $v$ for each particle. The distribution of particle velocities in the real experiment is taken account of by simply summing up the theoretical diffracted intensities obtained for the single particle velocities. This is possible because the particles in the beam propagate independently and thus exhibit no effects of coherence.

The concept of single velocities just suits the approach of stationary scattering theory whose central advantage lies in the fact that the scattered intensity in an experiment can be predicted by merely solving the time-independent Schrödinger equation

$$
H\left|\psi_{\mathbf{k}}\right\rangle=E\left|\psi_{\mathbf{k}}\right\rangle,
$$

for an energy eigenstate $\left|\psi_{\mathbf{k}}\right\rangle$, that is subject to the asymptotic condition

$$
\left\langle\mathbf{r} \mid \psi_{\mathbf{k}}\right\rangle \equiv \psi_{\mathbf{k}}(\mathbf{r}) \stackrel{z \ll 0}{\longrightarrow}\langle\mathbf{r} \mid \mathbf{k}\rangle \equiv \frac{1}{2 \pi} \mathrm{e}^{\mathrm{ikr}} .
$$

The energy $E$ associated with the energy eigenstate $\left|\psi_{\mathbf{k}}\right\rangle$ in Eq. (2.5) is determined in the region near the source where the scattering potential $V$ vanishes so that the full Hamiltonian $H$ can be replaced by the free Hamiltonian $H_{0}$, which thus represents the kinetic energy of the atom just leaving the source. In coordinate space representation one obtains for $H_{0}$ acting on the asymptotic state of Eq. (2.6)

$$
H_{0} \frac{1}{2 \pi} \mathrm{e}^{\mathrm{i} \mathbf{k r}}=\frac{(\hbar / \mathrm{i} \boldsymbol{\nabla})^{2}}{2 m} \frac{1}{2 \pi} \mathrm{e}^{\mathrm{i} \mathbf{k r}}=\frac{\hbar^{2} k^{2}}{2 m} \frac{1}{2 \pi} \mathrm{e}^{\mathrm{i} \mathbf{k r}}=E \frac{1}{2 \pi} \mathrm{e}^{\mathrm{i} \mathbf{k r}} .
$$


Rewriting and re-arranging Eq. (2.5) with this result leads to

$$
\left(\Delta+k^{2}-U(\mathbf{r})\right) \psi_{\mathbf{k}}(\mathbf{r})=0
$$

where for convenience the reduced potential $U:=2 m V / \hbar^{2}$ is introduced, which has the physical dimension of $\mathrm{m}^{-2}$.

Formally, the root of the inhomogeneous Eq. (2.8) is given by

$$
\psi_{\mathbf{k}}(\mathbf{r})=\frac{1}{2 \pi} \mathrm{e}^{\mathrm{ikr}}+\int \mathrm{d}^{2} r^{\prime} G\left(\mathbf{r}, \mathbf{r}^{\prime}\right) \psi_{\mathbf{k}}\left(\mathbf{r}^{\prime}\right) U\left(\mathbf{r}^{\prime}\right)
$$

The Green function $G\left(\mathbf{r}, \mathbf{r}^{\prime}\right)$, whose $k$ dependence is suppressed in the notation, is subject to

$$
\left(\Delta_{\mathbf{r}}+k^{2}\right) G\left(\mathbf{r}, \mathbf{r}^{\prime}\right)=\delta^{(2)}\left(\mathbf{r}-\mathbf{r}^{\prime}\right) .
$$

A closer look at the analytical properties of $G\left(\mathbf{r}, \mathbf{r}^{\prime}\right)$ reveals that in accordance with the incident wave function Eq. (2.6) it must be chosen such as to make $\psi_{\mathbf{k}}(\mathbf{r}) \mathrm{e}^{-\mathrm{i} \frac{E t}{\hbar}}, 0 \ll z$ a wavefunction going out from rather than running towards the scattering center [28, 32]. This property is indicated formally by the superscripts $\psi_{\mathbf{k}}^{(+)}(\mathbf{r})$ and $G^{(+)}\left(\mathbf{r}, \mathbf{r}^{\prime}\right)$.

In three-dimensional space one obtains for the Green function $\tilde{G}^{(+)}\left(\tilde{\mathbf{r}}, \tilde{\mathbf{r}}^{\prime}\right)$, corresponding to the three-dimensional outgoing solution $\tilde{\psi}_{\mathbf{k}}^{(+)}(\mathbf{r})$ of Eq. (2.9), the well-known result

$$
\tilde{G}^{(+)}\left(\tilde{\mathbf{r}}, \tilde{\mathbf{r}}^{\prime}\right)=-\frac{1}{4 \pi} \frac{\mathrm{e}^{\mathrm{i} k\left|\tilde{\mathbf{r}}-\tilde{\mathbf{r}}^{\prime}\right|}}{\left|\tilde{\mathbf{r}}-\tilde{\mathbf{r}}^{\prime}\right|},
$$

whereas in two dimensions the suitable Green function $G^{(+)}\left(\mathbf{r}, \mathbf{r}^{\prime}\right)$ is found according to Rubinowicz [33] by integrating the right-hand-side of Eq. (2.11) along the $y^{\prime}$ axis. With the substitutions $\tilde{\mathbf{r}}-\tilde{\mathbf{r}}^{\prime}=: \mathbf{R}=:\left(0, y-y^{\prime}, 0\right)+\varrho$ one has

$$
\tilde{G}^{(+)}\left(\mathbf{r}, \mathbf{r}^{\prime}\right)=-\frac{1}{4 \pi} \int_{-\infty}^{+\infty} \mathrm{d} y^{\prime} \frac{\mathrm{e}^{\mathrm{i} k R}}{R}
$$

This integral can be related to Sommerfeld's representation of the Hankel function $H_{0}^{(1)}$ of the first kind and order zero [35]

$$
H_{0}^{(1)}(z)=\int_{-\eta+\mathrm{i} \infty}^{\eta-\mathrm{i} \infty} \mathrm{d} t \mathrm{e}^{i z \cos t}, \quad 0 \leq \eta \leq \pi .
$$

After introducing to Eq. (2.12) the variable $t \in \mathbb{C}$ by

$$
y-y^{\prime}=:-\mathrm{i} \varrho \sin t, \quad \mathrm{~d} y^{\prime}=\mathrm{i} \varrho \cos t \mathrm{~d} t \Rightarrow R^{2}=\varrho^{2} \cos ^{2} t
$$


there follows as a result for the two-dimensional Green function

$$
G^{(+)}\left(\mathbf{r}, \mathbf{r}^{\prime}\right)=-\frac{\mathrm{i}}{4} H_{0}^{(1)}(k \varrho)
$$

Rewriting Eq. (2.9) with the additional information about the outgoing wavefunction leads to the two-dimensional Lippmann-Schwinger equation

$$
\psi_{\mathbf{k}}^{(+)}(\mathbf{r})=\frac{1}{2 \pi} \mathrm{e}^{\mathrm{i} \mathbf{k r}}+\int \mathrm{d}^{2} r^{\prime} G^{(+)}\left(\mathbf{r}, \mathbf{r}^{\prime}\right) \psi_{\mathbf{k}}^{(+)}\left(\mathbf{r}^{\prime}\right) U\left(\mathbf{r}^{\prime}\right)
$$

Far behind the grating, in the experiment near the detector, Eq. (2.16) can be expanded with respect to small $r^{\prime}$. The Hankel function from Eq. (2.15) is thereby replaced by its asymptotic behaviour [34]

$$
H_{0}^{(1)}(k \varrho) \stackrel{0 \ll k \varrho}{\longrightarrow} \sqrt{\frac{2}{\pi k \varrho}} \mathrm{e}^{\mathrm{i} k \varrho-\mathrm{i} \pi / 4}\left(1+\mathcal{O}\left(\frac{1}{k \varrho}\right)\right) .
$$

To first order in $r^{\prime}$, this yields

$$
\psi_{\mathbf{k}}^{(+)}(\mathbf{r}) \stackrel{0 \ll z}{\longrightarrow} \frac{1}{2 \pi} \mathrm{e}^{\mathrm{i} \mathbf{k r}}-\mathrm{i} \frac{\mathrm{e}^{i k r}}{\sqrt{r}} \frac{\sqrt{\lambda} \mathrm{e}^{-\frac{\mathrm{i} \pi}{4}}}{4 \pi} \int \mathrm{d}^{2} r^{\prime} \mathrm{e}^{-\mathrm{i} k \frac{\mathbf{r} \mathbf{r}^{\prime}}{r}} \psi_{\mathbf{k}}^{(+)}\left(\mathbf{r}^{\prime}\right) U\left(\mathbf{r}^{\prime}\right)
$$

with the de Broglie wavelength $\lambda:=\frac{2 \pi}{k}$. One recognizes in the first term the incoming plane wave, and in the second a circular wave going out from the origin of the coordinate frame where the scattering potential is located. The vector $k \frac{\mathbf{r}}{r}=: \mathbf{K}$ reflects the fact that the outgoing circular wave looks like a plane wave $\mathrm{e}^{\mathrm{i} \mathbf{K r}}$ as it reaches the far-away position $\mathbf{r}$ of the detector.

The two-dimensional scattering amplitude is defined by

$$
f(\mathbf{r}):=-\mathrm{i} \frac{\sqrt{\lambda} \mathrm{e}^{-\frac{\mathrm{i} \pi}{4}}}{4 \pi} \int \mathrm{d}^{2} r^{\prime} \mathrm{e}^{-\mathrm{i} \mathbf{K} \mathbf{r}^{\prime}} \psi_{\mathbf{k}}^{(+)}\left(\mathbf{r}^{\prime}\right) U\left(\mathbf{r}^{\prime}\right)
$$

Hereby, Eq. (2.18) becomes

$$
\psi_{\mathbf{k}}^{(+)}(\mathbf{r}) \stackrel{0 \ll z}{\longrightarrow} \frac{1}{2 \pi} \mathrm{e}^{\mathrm{ikr}}+\frac{\mathrm{e}^{i k r}}{\sqrt{r}} f(\mathbf{r}) .
$$

This result can be compared with the experimental count rate as a function of the detector position $\mathbf{r}$ which is often stated in terms of the scattering angle $\vartheta$ with

$$
\mathbf{r} \equiv(r \sin \vartheta, r \cos \vartheta)^{\mathrm{T}} .
$$

The theoretical equivalent of the experimental count rate is the probability $I_{\Omega}(\mathbf{r}) \equiv$ $I_{\Omega}(\vartheta)$ of an atom entering the detector aperture at $\mathbf{r}$ which subtends an angle $\Omega$ with respect to the scattering centre. $I_{\Omega}(\vartheta)$ is given by the probability flux 
through the aperture, which is just the integral of the well-known probability flux density over the angle $\Omega$. One has

$$
I_{\Omega}(\vartheta)=\frac{\hbar}{2 m \mathrm{i}} \int_{\vartheta-\frac{\Omega}{2}}^{\vartheta+\frac{\Omega}{2}} \mathrm{~d} \vartheta^{\prime} r\left(\left(\psi_{\mathbf{k}}^{(+)}\right)^{*}\left(\mathbf{r}^{\prime}\right) \frac{\partial}{\partial r} \psi_{\mathbf{k}}^{(+)}\left(\mathbf{r}^{\prime}\right)-\psi_{\mathbf{k}}^{(+)}\left(\mathbf{r}^{\prime}\right) \frac{\partial}{\partial r}\left(\psi_{\mathbf{k}}^{(+)}\right)^{*}\left(\mathbf{r}^{\prime}\right)\right),
$$

where a star has been used to denote the complex conjugate, and with $\mathbf{r}^{\prime} \equiv$ $\left(r \sin \vartheta^{\prime}, r \cos \vartheta^{\prime}\right)^{\mathrm{T}}$. Summing up the steps made so far, the important theoretical result is $I_{\Omega}(\mathbf{r})$ from Eq. (2.22) because it can be compared to experimental count rates of atoms scattered towards the detector position $\mathbf{r}$. In order to calculate $I_{\Omega}(\mathbf{r})$ from Eq. (2.22) the wavefunction $\psi_{\mathbf{k}}^{(+)}(\mathbf{r})$ is required for which, according to Eq. (2.20), one needs to know the scattering amplitude $f(\mathbf{r})$.

\subsection{Grating Potential}

To determine the scattering amplitude $f$ from Eq. (2.19) it is useful to discuss the structure of the scattering potential $U$, because then an appropriate approximation can be chosen that simplifies the remaining steps. The atom-surface potential consists of a strongly repulsive core (Pauli) and an attractive part (van der Waals) the latter of which will be shown in chapter 3 to be essentially given by $-\frac{C_{3}}{l^{3}}$, with the interaction strength $C_{3}>0$ and where $l$ stands for the distance between the atom and the surface. For the repulsive part there is a number of models available in the literature [36, 37] which are usually preferred to one another for reasons of mathematical convenience. The present problem is most simply described by a Lennard-Jones-type 9-3 potential

$$
V(l)=\frac{C_{9}}{l^{9}}-\frac{C_{3}}{l^{3}},
$$

with $C_{9}>0$. To give a quantitative example the approximate potential for ground-state helium atoms and a dielectric silicon nitride surface is plotted in Fig. 2.2. For systematic reasons, the scattering potential $U$ can be divided up into the comparatively weak attractive interaction $U^{\text {att }}$ and the very strong repulsive part $U^{\text {rep }}$.

To be definite, consider the closed line around the trapezoid-shaped crosssection of each bar in the $x-z$ plane (see the inset of Fig. 2.1) where the attractive and the repulsive part of the potential just cancel, like at $l_{0}$ in Fig. 2.2. If $\mathcal{A} \subset \mathbb{R}^{2}$ is the region within the circumferences so defined then one may write approximately $U=U^{\text {att }}+U^{\text {rep }}$ and

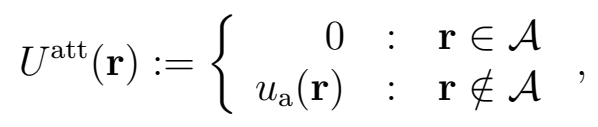




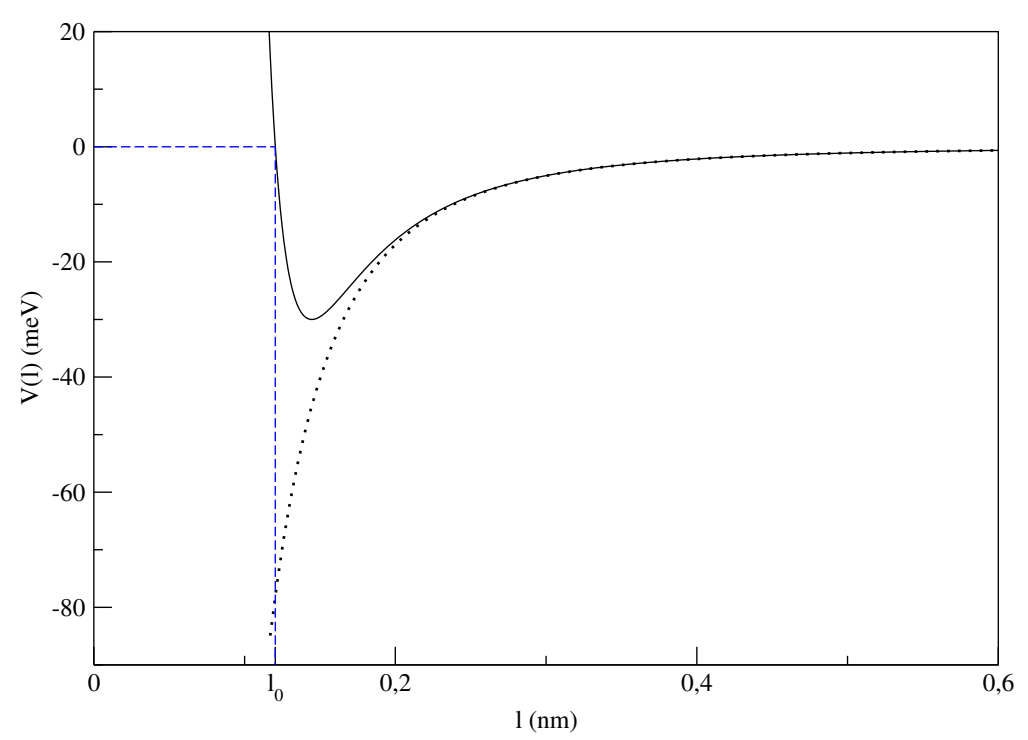

Figure 2.2: Realistically scaled sketch of the potential $V(l)$ (solid line) between a helium atom at a distance $l$ from a dielectric silicon nitride surface. At $l_{0}$ the repulsive and the attractive terms cancel (dashed lines). The attractive part alone is shown as a dotted line. The potential can be approximated by the sum of a repulsive square potential barrier for distances smaller than $l_{0}$ and the attractive part alone for distances greater than $l_{0}$.

where $u_{\mathrm{a}}(\mathbf{r})$ is an attractive potential and

$$
U^{\mathrm{rep}}(\mathbf{r}):=\left\{\begin{array}{rll}
u_{0} & : & \mathbf{r} \in \mathcal{A} \\
0 & : & \mathbf{r} \notin \mathcal{A}
\end{array},\right.
$$

with $u_{0} \gg E>0$.

From the range of the forces involved it is known that the potential of each grating bar practically vanishes on the sites of the neighbouring bars, hence the total grating potential $U$ can be written as a sum of single bar potentials

$$
U=\sum_{j} U_{j}
$$

all of which are, in the same sense as indicated in Eq. (2.24) and Eq. (2.25), the sum of an attractive and a repulsive part

$$
U_{j}=U_{j}^{\mathrm{att}}+U_{j}^{\mathrm{rep}}
$$

In absence of the attractive interaction $U^{\text {att }}$ the situation would be in complete analogy with the diffraction of light by a transmission grating with reflecting bar 
surfaces. For small incident wavelengths and correspondingly small diffraction angles and detector positions far behind the grating it is of minor importance if the bar surfaces are reflecting or absorbing. It has been shown by T. Köhler [24] that within the stated limits for a purely repulsive potential the reflected amplitude is negligible, which means that seen from far away only the silhouette of the grating contributes to the diffraction image. Therefore the case without any attractive potential is analogous to the diffraction of light from a thin transmission grating.

For atoms whose de Broglie wavelength $\lambda$ is small compared to the range $a$ of the bar potentials and if both $\lambda$ and $a$ are smaller than the slit width $s$ of the grating the probability of atoms being multiply scattered from several grating bars becomes negligibly small. A rigorous proof of this fact is based on the application of Watson's theorem [27, 28] for the scattering amplitude arising from a potential which is the sum of single potentials, like the grating bars in the present case [24]. As an important result it turns out that the scattering amplitude $f$ of the whole grating is well approximated by the sum of scattering amplitudes $f_{j}$ arising from the single bars, hence one has

$$
f=\sum_{j} f_{j}
$$

A similar treatment can be applied to the single bar potential which is the sum of an attractive and a repulsive part. In the limit of small de Broglie wavelengths it can be deduced from Watson's theorem that also for the single grating bar potential the total scattering amplitude is well approximated by the sum of scattering amplitudes $f_{j}^{\text {att }}$ and $f_{j}^{\text {rep }}$ arising from the respective attractive and repulsive parts alone [24]. Hereby the scattering amplitude of the attractive potential is calculated with respect to an incident wave that has been diffracted by the repulsive part of the potential, a common procedure which is known as the distorted wave Born approximation $[27,28]$. Bearing this in mind one may write

$$
f=\sum_{j} f_{j}=\sum_{j}\left(f_{j}^{\text {att }}+f_{j}^{\text {rep }}\right)
$$

As the scattering from the repulsive part of the grating potential can be reduced to the simple analogy with the diffraction of light it is possible to calculate the detection probability by an easy scheme that nevertheless fully includes the concept of the distorded wave Born approximation. This scheme is formally accomplished by replacing in equation Eq. (2.20) the general scattering amplitude $f(\mathbf{r})$ by the more detailed expression Eq. (2.29) which yields

$$
\psi_{\mathbf{k}}^{(+)}(\mathbf{r}) \stackrel{0 \ll z}{\longrightarrow} \frac{1}{2 \pi} \mathrm{e}^{\mathrm{ikr}}+\frac{\mathrm{e}^{i k r}}{\sqrt{r}} \sum_{j}\left(f_{j}^{\mathrm{att}}(\mathbf{r})+f_{j}^{\mathrm{rep}}(\mathbf{r})\right) .
$$


After identifying the wavefunction $\psi_{\text {rep }}^{(+)}(\mathbf{r})$ arising from the scattering by the repulsive potential alone one has

$$
\psi_{\mathbf{k}}^{(+)}(\mathbf{r}) \stackrel{0 \ll z}{\longrightarrow} \psi_{\text {rep }}^{(+)}(\mathbf{r})+\frac{\mathrm{e}^{i k r}}{\sqrt{r}} \sum_{j} f_{j}^{\text {att }}(\mathbf{r}) .
$$

The advantages of this procedure are that $\psi_{\text {rep }}^{(+)}(\mathbf{r})$ can be easily calculated with a method known from optics [32] which is based on the application of Green's theorem, to be evaluated here in two dimensions. Furthermore, the result to be obtained contains two terms that cancel exactly which yields an expression for the detection probability that can be generalized straightforwardly to include Fresnel diffraction effects. As a first step, the wavefunction $\psi_{\text {rep }}^{(+)}(\mathbf{r})$ that describes the diffraction of atoms from the repulsive core of the grating bar potentials is calculated in the following section.

\subsection{Diffraction from the Repulsive Potential}

As explained above, the influence of the repulsive potential on the diffraction of atoms from a transmission grating can be reduced to the simple case that the transmission grating is represented by a plane screen perforated by a periodic array of slits. The wavefunction $\psi_{\mathrm{rep}}^{(+)}(\mathbf{r})$ at a (detector) position $\mathbf{r}$ behind the grating is subject to the Helmholtz equation

$$
\left(\Delta+k^{2}\right) \psi_{\text {rep }}^{(+)}(\mathbf{r})=0 .
$$

This is just the formerly stated Eq. (2.8), for the given case of the grating potential $U$ being zero everywhere except right on the grating bars. Drawing a small circle $\partial C$ around the position $\mathbf{r}$ of the detector one may define a region $A \subset \mathbb{R}^{2}$ as the whole region behind the grating except for the area $C$ of the small circle.

Fig. 2.4 illustrates how the concept of this region $A$ is realized by mounting a semicircle on the back side of the screen whose perforation constitutes the transmission grating. Letting the radius of this semicircle tend to infinity accomplishes the region $A$. The fact that the incident wave (from the left, in the figure) is totally blocked by the screen outside the perforated stretch is in agreement with the experimental situation where a collimated beam is used that vanishes outside a certain illuminated spot on the grating.

Using Eq. (2.32) and the definition Eq. (2.10) of the appropriate two-dimensional Green function one notes that inside $A$ the equation

$$
\int_{A} \mathrm{~d}^{2} \mathbf{r}^{\prime}\left(G\left(\mathbf{r}, \mathbf{r}^{\prime}\right) \Delta \psi_{\text {rep }}^{(+)}\left(\mathbf{r}^{\prime}\right)-\psi_{\text {rep }}^{(+)}\left(\mathbf{r}^{\prime}\right) \Delta G\left(\mathbf{r}, \mathbf{r}^{\prime}\right)\right)=0
$$




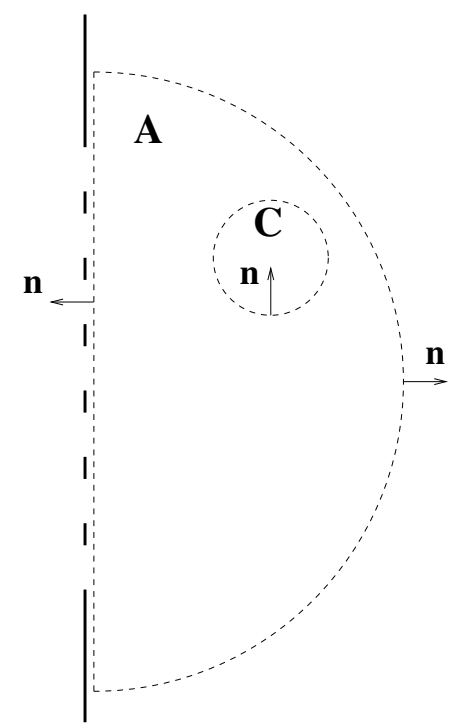

Figure 2.3: The area $A$ is bounded by the rear side of the perforated screen that represents the transmission grating, and by a semicircle mounted thereon. Note that $A$ excludes the circle $C$ around the detector position behind the grating. The incident atoms arrive at the screen from the left. The normal vectors on the boundary $\partial A$ are defined to point out of the region $A$.

holds. On applying Green's theorem in two dimensions this area integral can be converted into a line integral along the boundaries $\partial A$ of the region $A$ which are the infinite semicircle and the small circle around the detector position $\mathbf{r}$. One has

$$
0=\int_{\partial A} \mathrm{~d} s\left(\mathbf{r}^{\prime}\right)\left(G\left(\mathbf{r}, \mathbf{r}^{\prime}\right) \frac{\partial}{\partial \mathbf{n}} \psi_{\text {rep }}^{(+)}\left(\mathbf{r}^{\prime}\right)-\psi_{\text {rep }}^{(+)}\left(\mathbf{r}^{\prime}\right) \frac{\partial}{\partial \mathbf{n}} G\left(\mathbf{r}, \mathbf{r}^{\prime}\right)\right),
$$

where the normal vector $\mathbf{n}$ points out of the region $A$ as indicated in Fig. 2.4. On the infinite semicircle, the integrand vanishes except on the openings of the screen which can be seen as follows. For large $r$ the Green function, which is a Hankel function as introduced in Eq. (2.15) and Eq. (2.17), is proportional to $\frac{1}{\sqrt{r}}$ and so is the wavefunction $\psi_{\text {rep }}^{(+)}(\mathbf{r})$ because from Huygens' principle it is expected to be a cylindrical wave with the stated $r$ dependence. Each term of the integrand in Eq. (2.34) is therefore, with inclusion of the spatial derivative, proportional to $\frac{1}{r^{2}}$. On the arc of the semicircle which is linear in $r$ this leads to a vanishing like $\frac{1}{r}$ of the respective contributions to the line integral as the arc tends to infinite radii. The wavefunction $\psi_{\text {rep }}^{(+)}(\mathbf{r})$ also vanishes on $\partial A$ immediately behind the grating bars which obstruct the plane wave that falls on the grating from the left.

The contribution of the small circle around the detector position $\mathbf{r}$ to the line integral Eq. (2.34) is evaluated in the limit of infinitely small radii $R_{0}:=\left|\mathbf{r}-\mathbf{r}^{\prime}\right| \rightarrow$ 
0 of the circle. As a result, one has

$$
\lim _{R_{0} \rightarrow 0} \int_{\partial C} \mathrm{~d} s\left(\mathbf{r}^{\prime}\right)\left(G\left(\mathbf{r}, \mathbf{r}^{\prime}\right) \frac{\partial}{\partial \mathbf{n}} \psi_{\text {rep }}^{(+)}\left(\mathbf{r}^{\prime}\right)-\psi_{\text {rep }}^{(+)}\left(\mathbf{r}^{\prime}\right) \frac{\partial}{\partial \mathbf{n}} G\left(\mathbf{r}, \mathbf{r}^{\prime}\right)\right)=\psi_{\text {rep }}^{(+)}(\mathbf{r}) .
$$

To see how this comes about consider first the second term $\psi_{\text {rep }}^{(+)}\left(\mathbf{r}^{\prime}\right) \frac{\partial}{\partial \mathbf{n}} G\left(\mathbf{r}, \mathbf{r}^{\prime}\right)$ in Eq. (2.34). In the limit $R_{0} \rightarrow 0$ the Green function $\mathrm{G}$ is known to behave like $G \rightarrow \frac{1}{2 \pi} \ln \left(\frac{R_{0}}{2}\right)[33]$ which leads to

$$
\lim _{R_{0} \rightarrow 0} \psi_{\text {rep }}^{(+)}\left(\mathbf{r}^{\prime}\right) \frac{\partial}{\partial \mathbf{n}} G\left(\mathbf{r}, \mathbf{r}^{\prime}\right)=-\psi_{\text {rep }}^{(+)}\left(\mathbf{r}^{\prime}\right) \frac{1}{2 \pi R_{0}} .
$$

Upon line integration around the circle $\partial C$ this becomes $-\psi_{\text {rep }}^{(+)}(\mathbf{r})$. The first term in the integrand of Eq. (2.35) vanishes in the limit $R_{0} \rightarrow 0$ because $\frac{\partial}{\partial \mathbf{n}} \psi_{\text {rep }}^{(+)}\left(\mathbf{r}^{\prime}\right)$ is finite and the circumference of the circle is linear in $R_{0}$ so that the logarithmic divergence of $G$ is overridden.

Therefore, in order to find the solution $\psi_{\text {rep }}^{(+)}(\mathbf{r})$ of the diffraction problem it suffices in Eq. (2.34) to calculate the remaining part of the contour $\partial A$ along the openings of the screen. It is evaluated with the help of the asymptotic behaviour Eq. (2.17) for large arguments of the Green function Eq. (2.15) and yields

$$
\psi_{\text {rep }}^{(+)}(\mathbf{r})=\frac{1}{2 \pi} \frac{\mathrm{e}^{\mathrm{i} k r}}{\sqrt{r}} \frac{\mathrm{e}^{-\mathrm{i} \pi / 4}}{\sqrt{\lambda}} \sum_{j} \int_{-\frac{s}{2}}^{\frac{s}{2}} \mathrm{~d} x^{\prime} \mathrm{e}^{-\mathrm{i} k\left(x^{\prime}+j d\right) \sin \vartheta} .
$$

By the summing over $j$ the integration runs over each grating slit, in that the integrand contains an appropriate shift of the local coordinate $x^{\prime}$ parallel to the backside of the grating by $j$ times the grating period $d$.

\subsection{Eikonal Approximation}

As the attractive contribution $U^{\text {att }}$ to the total scattering potential $U$ is relatively small, and the observation in the experiment is restricted to small scattering angles, the scattering amplitudes $f_{j}^{\text {att }}$ which arise from the attractive potential in the $j$ th slit are calculated in the semi-classical eikonal approximation which is especially suitable for these cases [28]. As mentioned earlier the scattering amplitudes arising from the attractive potential are calculated for an incident wavefunction $\psi_{\text {rep }}^{(+)}(\mathbf{r})$ that is already diffracted by the repulsive part of the potential. The eikonal approximation yields a result for $f_{j}^{\text {att }}$ that only depends on the incident wavefunction at positions inside the $j$ th slit. Applying Huygens' principle to the diffraction by the repulsive potential alone which is analogous to the diffraction 
of light is is clear that inside the slits the wavefunction $\psi_{\text {rep }}^{(+)}\left[(x, 0)^{\mathrm{T}}\right]$ is identical to the incident plane wave.

This motivates the ansatz

$$
\psi_{\mathbf{k}}^{(+)}(\mathbf{r})=: \frac{\mathrm{e}^{\mathrm{ikr}}}{2 \pi} \varphi_{\mathbf{k}}(\mathbf{r})
$$

which stands at the beginning of the procedure leading to the scattering amplitude $f_{j}^{\text {att }}$ in eikonal approximation. Inserting the ansatz into Eq. (2.16) one has

$$
\varphi_{\mathbf{k}}(\mathbf{r})=1-\int \mathrm{d}^{2} r^{\prime} G^{(+)}\left(\mathbf{r}, \mathbf{r}^{\prime}\right) U_{j}^{\mathrm{att}}\left(\mathbf{r}^{\prime}\right) \mathrm{e}^{\mathrm{i} \mathbf{k}\left(\mathbf{r}^{\prime}-\mathbf{r}\right)} \varphi_{\mathbf{k}}\left(\mathbf{r}^{\prime}\right) .
$$

For the following steps it is instructive to look in detail at the term $G^{(+)}\left(\mathbf{r}, \mathbf{r}^{\prime}\right)$. Upon Fourier transformation the definition Eq. (2.10) of the Green function in terms of a $\delta$-function reads

$$
\frac{1}{(2 \pi)^{2}} \int \mathrm{d}^{2} \kappa \mathrm{e}^{\mathrm{i} \boldsymbol{\kappa}\left(\mathbf{r}-\mathbf{r}^{\prime}\right)}=\left(\Delta_{\mathbf{r}}+k^{2}\right) \frac{1}{(2 \pi)^{2}} \int \mathrm{d}^{2} \kappa \mathrm{e}^{\mathrm{i} \boldsymbol{\kappa}\left(\mathbf{r}-\mathbf{r}^{\prime}\right)} \breve{G}(\boldsymbol{\kappa}),
$$

which by standard methods leads to

$$
G\left(\mathbf{r}, \mathbf{r}^{\prime}\right)=\frac{-1}{(2 \pi)^{2}} \int \mathrm{d}^{2} \kappa \frac{\mathrm{e}^{\mathrm{i} \kappa\left(\mathbf{r}-\mathbf{r}^{\prime}\right)}}{\kappa^{2}-k^{2}}=\int_{0}^{2 \pi} \frac{\mathrm{d} \varphi}{(2 \pi)^{2}} \int_{-\infty}^{+\infty} \mathrm{d} \kappa\left(\frac{\kappa}{\kappa-k}-\frac{\kappa}{\kappa+k}\right) \mathrm{e}^{\mathrm{i} \kappa\left|\mathbf{r}-\mathbf{r}^{\prime}\right| \cos \varphi}
$$

The outgoing wave solution Eq. (2.11) requires a complex contour integration along the real axis and a half-circle in the upper half-plane during which the poles at $\pm k$ are infinitesimally shifted to $\pm(k+\mathrm{i} \varepsilon)$. Formally, this procedure is denoted by

$$
G^{(+)}\left(\mathbf{r}, \mathbf{r}^{\prime}\right)=\frac{-1}{(2 \pi)^{2}} \int \mathrm{d}^{2} \kappa \frac{\mathrm{e}^{\mathrm{i} \kappa\left(\mathbf{r}-\mathbf{r}^{\prime}\right)}}{\kappa^{2}-k^{2}-\mathrm{i} \varepsilon} .
$$

With Eq. (2.42) and the new variables $\mathbf{K}:=\boldsymbol{\kappa}-\mathbf{k}$ and $\mathbf{R}:=\mathbf{r}-\mathbf{r}^{\prime}$ Eq. (2.39) is rewritten as

$$
\varphi_{\mathbf{k}}^{(+)}(\mathbf{r})=1-\frac{1}{(2 \pi)^{2}} \int \mathrm{d}^{2} R \int \mathrm{d}^{2} K \frac{\mathrm{e}^{\mathrm{i} \mathbf{K R}}}{K^{2}+2 \mathbf{K} \mathbf{k}-\mathrm{i} \varepsilon} U_{j}^{\mathrm{att}}(\mathbf{r}-\mathbf{R}) \varphi_{\mathbf{k}}^{(+)}(\mathbf{r}-\mathbf{R}) .
$$

Examining the integrand with respect to $\int \mathrm{d}^{2} R$ it becomes evident that the integral becomes stationary for small values of $K$. It is therefore reasonable to expand the first term of the integrand in Eq. (2.43) with respect to small $K$ which yields

$$
\frac{\mathrm{e}^{\mathrm{i} \mathbf{K R}}}{K^{2}+2 \mathbf{K k}-\mathrm{i} \varepsilon}=\frac{\mathrm{e}^{\mathrm{i} \mathbf{K R}}}{2 \mathbf{K} \mathbf{k}-\mathrm{i} \varepsilon}\left(1+\frac{K^{2}}{2 \mathbf{K k}-\mathrm{i} \varepsilon}+\mathcal{O}\left(K^{4}\right)\right) .
$$

This expansion forms the core of the eikonal approximation. It is possible to proceed in lowest order if $k a \gg 1$, i.e. the de Broglie wavelength $\lambda:=\frac{2 \pi}{k}$ of the 
atoms be much shorter than the range $a$ of the attractive potential. In a typical experimental situation the de Broglie wavelengths $\lambda$ involved are about $50 \mathrm{pm}$, while the van der Waals interaction of the atoms with the bars has a typical range of about $1 \mathrm{~nm}$. This leads to $k a \approx 100$.

Proceeding from Eq. (2.44) in zeroth order Eq. (2.43) becomes after a little algebra

$$
\varphi_{\mathbf{k}}^{(+)}(\mathbf{r})=1-\frac{1}{4 \pi k} \int \mathrm{d}^{2} R \delta(X) U_{j}^{\mathrm{att}}(\mathbf{r}-\mathbf{R}) \varphi_{\mathbf{k}}^{(+)}(\mathbf{r}-\mathbf{R}) \int_{-\infty}^{+\infty} \mathrm{d} K_{z} \frac{\mathrm{e}^{\mathrm{i} K_{z} Z}}{2 K_{z} k-\mathrm{i} \varepsilon}
$$

where the $K_{z}$-axis has been chosen to point in the direction of the incident plane wave vector $\mathbf{k}$. By contour integration in the complex plane the second integral is zero for $Z \equiv z-z^{\prime}<0$, i.e. before the grating, and $2 \pi$ i for $Z>0$, i.e. behind the grating, in the region of interest. Thus, in zeroth order eikonal approximation one has $\varphi_{\mathbf{k}}^{(+)}(\mathbf{r}) \approx \varphi_{\mathbf{k}}^{\mathrm{E}}(\mathbf{r})$, where $\varphi_{\mathbf{k}}^{\mathrm{E}}(\mathbf{r})$ is subject to

$$
\varphi_{\mathbf{k}}^{\mathrm{E}}(\mathbf{r})=1-\frac{\mathrm{i}}{2 k} \int_{0}^{+\infty} \mathrm{d} Z U_{j}^{\mathrm{att}}\left(\mathbf{r}-Z \hat{\mathbf{e}}_{\mathbf{z}}\right) \varphi_{\mathbf{k}}^{\mathrm{E}}\left(\mathbf{r}-Z \hat{\mathbf{e}}_{\mathbf{z}}\right)
$$

and $\hat{\mathbf{e}}_{\mathbf{z}}$ is the unit vector in $z$-direction. As one can easily check, Eq. (2.46) is solved by

$$
\varphi_{\mathbf{k}}^{\mathrm{E}}(\mathbf{r})=\exp \left(-\frac{\mathrm{i}}{2 k} \int_{-\infty}^{z} \mathrm{~d} z^{\prime} U_{j}^{\text {att }}\left(x, z^{\prime}\right)\right)
$$

Going back to the ansatz Eq. (2.38) one has for the zeroth order eikonal approximation of the wavefunction in the scattering region

$$
\psi_{\mathbf{k}}^{(+)}(\mathbf{r})=\frac{\mathrm{e}^{\mathrm{ikr}}}{2 \pi} \exp \left(-\frac{\mathrm{i}}{2 k} \int_{-\infty}^{z} \mathrm{~d} z^{\prime} U_{j}^{\text {att }}\left(x, z^{\prime}\right)\right)
$$

In order to obtain the scattering amplitude $f_{j}^{\text {att }}$ the result Eq. $(2.48)$ is inserted 
into Eq. (2.19) which yields

$$
\begin{aligned}
f_{j}^{\text {att }}(\mathbf{r}) & =\frac{\sqrt{\lambda} \mathrm{e}^{-\frac{\mathrm{i} \pi}{4}}}{4 \pi \mathrm{i}} \int \mathrm{d}^{2} r^{\prime} \mathrm{e}^{-\mathrm{i} \mathbf{K} \mathbf{r}^{\prime}} U_{j}^{\mathrm{att}}\left(\mathbf{r}^{\prime}\right) \frac{\mathrm{e}^{\mathrm{i} \mathbf{k r} \mathbf{r}^{\prime}}}{2 \pi} \exp \left(-\frac{\mathrm{i}}{2 k} \int_{-\infty}^{z^{\prime}} \mathrm{d} z^{\prime \prime} U_{j}^{\mathrm{att}}\left(x^{\prime}, z^{\prime \prime}\right)\right)= \\
& =\frac{\mathrm{e}^{-\frac{\mathrm{i} \pi}{4}}}{2 \pi \sqrt{\lambda}} \int \mathrm{d}^{2} r^{\prime} \mathrm{e}^{\mathrm{i}(\mathbf{k}-\mathbf{K}) \mathbf{r}^{\prime}} \frac{\mathrm{d}}{\mathrm{d} z^{\prime}} \exp \left(-\frac{\mathrm{i}}{2 k} \int_{-\infty}^{z^{\prime}} \mathrm{d} z^{\prime \prime} U_{j}^{\mathrm{att}}\left(x^{\prime}, z^{\prime \prime}\right)\right) \stackrel{\cos \vartheta \approx 1}{\approx}(2.50) \\
& \approx \frac{\mathrm{e}^{-\frac{\mathrm{i} \pi}{4}}}{2 \pi \sqrt{\lambda}} \int_{-\infty}^{+\infty} \mathrm{d} x^{\prime} \mathrm{e}^{-\mathrm{i} k x^{\prime} \sin \vartheta}\left\{\exp \left(-\frac{\mathrm{i}}{2 k} \int_{-\infty}^{\infty} \mathrm{d} z^{\prime \prime} U_{j}^{\text {att }}\left(x^{\prime}, z^{\prime \prime}\right)\right)-1\right\} .
\end{aligned}
$$

In the third step, the approximation for small diffraction angles $\vartheta$

$$
\mathbf{r}^{\prime}(\mathbf{k}-\mathbf{K})=\left(x^{\prime}, z^{\prime}\right) \cdot k\left(\begin{array}{c}
0-\sin \vartheta \\
1-\cos \vartheta
\end{array}\right) \approx-k x^{\prime} \sin \vartheta
$$

has been used. Recalling that the evaluation of $f_{j}^{\text {att }}(\mathbf{r})$ has to be in terms of the wavefunction $\psi_{\text {rep }}^{(+)}(\mathbf{r})$ that results from the influence of the repulsive potential it is clear that the integral along the back side of the grating that appears in Eq. (2.49) has to include the grating slits where $\psi_{\text {rep }}^{(+)}(\mathbf{r})$ is equal to the incident wave, and exclude the region right behind the bars where $\psi_{\text {rep }}^{(+)}(\mathbf{r})$ is zero.

It is convenient at this point to express the attractive potential of two neighbouring grating bars in terms of the attractive potential $V^{\text {att }}$ that is produced in the slit in between. After defining

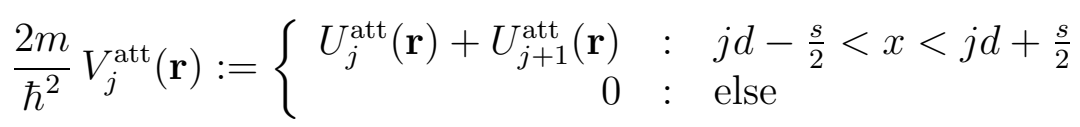

it can be assumed that the attractive potential is the same in each slit so that the subscript $j$ is dropped and $V_{j}^{\text {att }}(\mathbf{r}) \equiv V^{\text {att }}(\mathbf{r})$. The scattering amplitude $f^{\text {att }}(\mathbf{r})$ of all grating slits, as arising from the attractive interaction is obtained according to Eq. (2.28) and Eq. (2.29) by summing up the contributions of all slits. This yields

$$
f^{\mathrm{att}}(\mathbf{r})=\sum_{j} \frac{\mathrm{e}^{-\frac{\mathrm{i} \pi}{4}}}{2 \pi \sqrt{\lambda}} \int_{-\frac{s}{2}}^{+\frac{s}{2}} \mathrm{~d} x^{\prime} \mathrm{e}^{-\mathrm{i} k\left(x^{\prime}+j d\right) \sin \vartheta}\left\{\exp \left(-\frac{\mathrm{i}}{\hbar v} \int_{-\infty}^{\infty} \mathrm{d} z^{\prime \prime} V^{\mathrm{att}}\left(x^{\prime}, z^{\prime \prime}\right)\right)-1\right\} .
$$


In the limit of large distances $r$ from the grating to the detector and within the stated approximations the solution to the whole diffraction problem becomes

$$
\psi_{\mathbf{k}}^{(+)}(\mathbf{r})=\psi_{\text {rep }}^{(+)}(\mathbf{r})+\frac{\mathrm{e}^{i k r}}{\sqrt{r}} f^{\text {att }}(\mathbf{r}) .
$$

Inserting the results Eq. (2.37) for $\psi_{\text {rep }}^{(+)}(\mathbf{r})$ and Eq. (2.54) for $f^{\text {att }}(\mathbf{r})$ into Eq. (2.55) one obtains

$$
\psi_{\mathbf{k}}^{(+)}(\mathbf{r})=\sum_{j} \frac{\mathrm{e}^{i\left(k r-\frac{\pi}{4}\right)}}{2 \pi \sqrt{\lambda r}} \int_{-\frac{s}{2}}^{+\frac{s}{2}} \mathrm{~d} x^{\prime} \mathrm{e}^{-\mathrm{i} k\left(x^{\prime}+j d\right) \sin \vartheta} \exp \left(-\frac{\mathrm{i}}{\hbar v} \int_{-\infty}^{\infty} \mathrm{d} z^{\prime \prime} V^{\mathrm{att}}\left(x^{\prime}, z^{\prime \prime}\right)\right) .
$$

Introducing the momentum transfer

$$
\kappa:=k \sin \vartheta \quad \text {, or } \quad \kappa^{\prime}:=k \sin \vartheta^{\prime}
$$

and evaluating the sum for $N$ grating slits one has

$$
\sum_{j=0}^{N-1} \mathrm{e}^{-\mathrm{i} \kappa j d}=\frac{1-\mathrm{e}^{-\mathrm{i} \kappa N d / 2}}{1-\mathrm{e}^{-\mathrm{i} \kappa d / 2}}=\mathrm{e}^{-\mathrm{i} \kappa(N-1) d / 2} \frac{\sin (N \kappa d / 2)}{\sin (\kappa d / 2)} .
$$

According to Eq. (2.22), the diffraction of a monochromatic beam of velocity $v$ with an incident direction parallel to the $z$ axis leads to a detection probability

$$
I_{\Omega}(\vartheta)=\left.\frac{N v}{4 \pi^{2} \lambda} \int_{\vartheta-\frac{\Omega}{2}}^{\vartheta+\frac{\Omega}{2}} \mathrm{~d} \vartheta^{\prime} H_{N}\left(\kappa^{\prime}\right) \int_{-s / 2}^{+s / 2} \mathrm{~d} x^{\prime} \mathrm{e}^{-\mathrm{i} \kappa^{\prime} x^{\prime}} \exp \left(-\frac{\mathrm{i}}{\hbar v} \int_{-\infty}^{+\infty} \mathrm{d} z V^{\mathrm{att}}\left(x^{\prime}, z\right)\right)\right|^{2},
$$

with the grating function

$$
H_{N}(\kappa):=\frac{1}{N}\left|\mathrm{e}^{-\mathrm{i} \kappa(N-1) d / 2} \frac{\sin (N \kappa d / 2)}{\sin (\kappa d / 2)}\right|^{2}=\frac{\sin ^{2}(N \kappa d / 2)}{N \sin ^{2}(\kappa d / 2)} .
$$

Apparently, Eq. (2.59) is analogous to classical optics except for the additional phase factor

$$
\psi(x, 0):=\exp \left(-\frac{\mathrm{i}}{\hbar v} \int_{-\infty}^{+\infty} \mathrm{d} z V^{\mathrm{att}}(x, z)\right)
$$

that can be interpreted as being inscribed on the incoming plane wave at the position $z=0$, i.e. the back side of the grating. The phase factor not only accounts for the phase arising from the atom travelling through the attractive potential before it passes the grating but also through the region behind the grating 
on its way to the detector. As the scattering angles are small the integration is carried out along a straight line trajectory, regardless of the scattering angle. This is a central feature of the eikonal approximation.

It is interesting to see how the result Eq. (2.59) can be related to experimental diffraction patterns. As a first point, it is well known from optical diffraction theory that for large numbers $N$ of illuminated grating slits the grating function Eq. (2.60) turns into a "Dirac Comb"

$$
\lim _{N \rightarrow \infty} H_{N}(\kappa)=\pi \sum_{n=-\infty}^{+\infty} \delta\left(\kappa-\kappa_{n}\right)
$$

i.e. a row of equally spaced $\delta$-functions with a period determined by the zeros $\vartheta_{n}$ of the denominator in Eq. (2.60) which are given by

$$
\sin \vartheta_{n}=\frac{n \lambda}{d}
$$

so that $\kappa_{n}=\frac{2 \pi n}{d}$. If the grating function is assumed to be $\delta$-like then Eq. (2.59) is non-zero only for the principal diffraction angles $\vartheta_{n}$, namely

$$
I_{\Omega}\left(\vartheta_{n}\right)=\frac{N v}{8 \pi^{3}}\left|f_{\text {slit }}\left(\vartheta_{n}\right)\right|^{2},
$$

where a slit amplitude has been defined by

$$
f_{\text {slit }}(\vartheta):=\int_{-s / 2}^{+s / 2} \mathrm{~d} x^{\prime} \mathrm{e}^{-\mathrm{i} \kappa x^{\prime}} \exp \left(-\frac{\mathrm{i}}{\hbar v} \int_{-\infty}^{+\infty} \mathrm{d} z V^{\text {att }}\left(x^{\prime}, z\right)\right) .
$$

For finite $N$ the gaps between the principal maxima are populated with $N$ smaller side maxima, as the reader may recall from Fraunhofer diffraction of classical optics. In a typical experiment, one has $N \approx 100$ and so that roughly 8 side maxima additionally fall into the detector aperture $\Omega \approx 0.06 \mathrm{mrad}$ if the detector is placed centre on one of the principal maxima whose spacing is $\frac{\lambda}{d} \approx$ $0.5 \mathrm{mrad}$. The integral over the detector opening in Eq. (2.59) in this case also depends on how the series of maxima is modulated by the slit amplitude $\left|f_{\text {slit }}(\vartheta)\right|^{2}$. A numerical study reveals that for $N=100$ the detection probability $I_{\Omega}(\mathbf{r})$ at the principal diffraction angles is between $1.73 \%$ and $1.4 \%$ smaller than that given in Eq. (2.64) for $N \gg 100$. Bearing in mind that for a comparison with the experiment only the ratios of different diffraction orders are used, this nearly constant correction factor can be ignored as it leads to an error in the relative intensities of at most $0.33 \%$. Fig. 2.4 illustrates for the realistic case of $N=100$ the intensity across the detector aperture if it is placed centre on the zeroth diffraction order. 


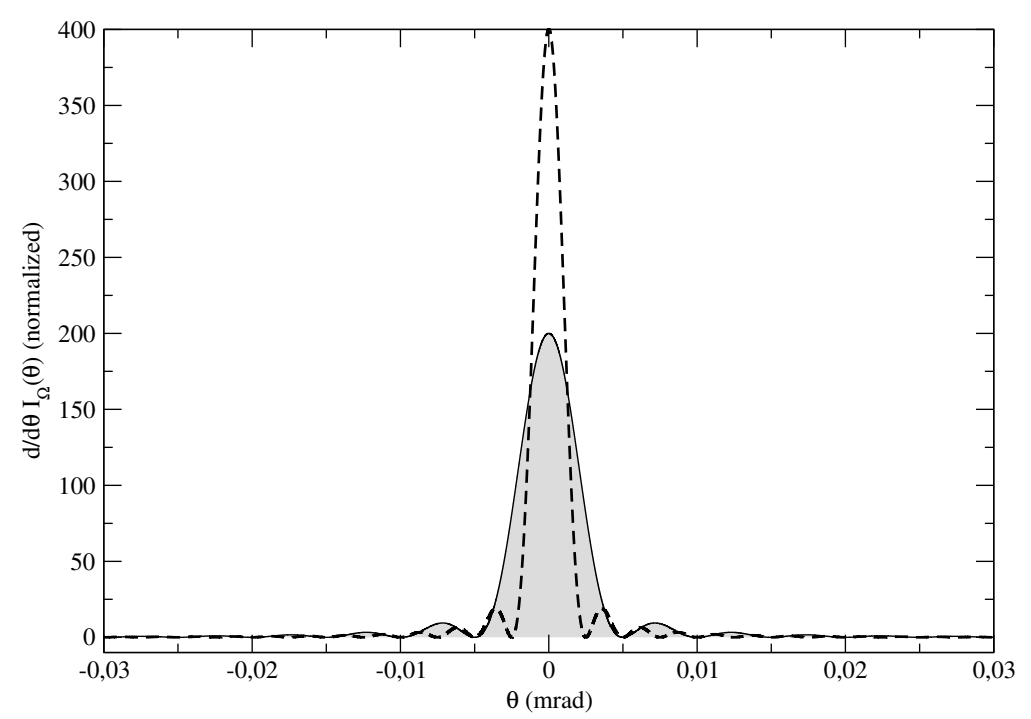

Figure 2.4: The theoretical detection probability for the zeroth diffraction order of a monochromatic incident wave is equal to the integral over the shown curves, which are the angle-dependent diffracted intensities as given inside the detector aperture which ranges over $0.06 \mathrm{mrad}$. The shaded area under the solid curve is calculated with $N=100$ illuminated grating slits. It is wider than the other curve calculated with $N=200$. The respective areas are 0.983 and 0.992 while in the limit of $N \rightarrow \infty$ the central peak becomes a delta function whose area is unity.

In the experiment, the measured diffraction pattern is interpreted as an incoherent superposition of many monochromatic diffraction patterns of the sort described by Eq. (2.59). They correspond each to a set of particles in the beam, while each set contains enough particles to represent a quantum mechanical ensemble. The observed shape of the principal orders is broadened as a consequence of the inevitable angular and velocity distribution of the atoms in the incident beam. As the monochromatic components are well described by $\delta$-shaped principal maxima and as long as the broadening does not merge the peaks of neighbouring order the relative areas $A_{\exp }(n)$ under the measured $n$th order peaks are equivalent to the relative intensities $I_{\Omega}\left(\vartheta_{n}\right)$ of the principal diffraction orders as given by Eq. (2.59). For example, normalizing to the zeroth order one has

$$
\frac{I_{\Omega}\left(\vartheta_{n}\right)}{I_{\Omega}(0)} \stackrel{!}{=} \frac{A_{\exp }(n)}{A_{\exp }(0)}
$$

With this formula a direct comparison of the theory with the experimental results can be established. 


\subsection{Fresnel Corrections}

For the real experiments it is necessary to generalize the present approach to include situations in which the detector is closer to the scattering centre than would justify the use of the Fraunhofer diffraction limit. This limit applies if the path difference $\Delta$ between two lines connecting the detector position with the centre or the boundary of the illuminated region on the transmission grating is small compared to the de Broglie wavelength $\lambda$ of the incident particles. In the typical experimental situation, where the detector slit is at a distance of $r=0.43 \mathrm{~m}$ from the grating which is illuminated over a width of about 100 periods $(=10 \mu \mathrm{m})$, one has $\Delta \approx 0.05 \mathrm{~nm}$ while also $\lambda \approx 0.05 \mathrm{~nm}$ so that the Fraunhofer limit is not readily justified.

In order to adjust this shortcoming an exact treatment of the path difference can be realized by replacing the Fraunhofer term $\mathrm{e}^{\mathrm{i}\left[k r-\kappa\left(x^{\prime}+j d\right)\right]}$ in Eq. (2.56) with the exact expression

$$
\mathrm{e}^{\mathrm{i} k \sqrt{\left(x-x^{\prime}-j d\right)^{2}+z^{2}}}
$$

where $x$ and $z$ are the coordinates of the detector. After expansion of the square root with respect to small $\left(x^{\prime}+j d\right)$, Eq. (2.56) becomes

$$
\psi_{\mathbf{k}}^{(+)}(\mathbf{r})=\sum_{j} \frac{\mathrm{e}^{i\left(k r-\frac{\pi}{4}\right)}}{2 \pi \sqrt{\lambda r}} \int_{-\frac{s}{2}}^{+\frac{s}{2}} \mathrm{~d} x^{\prime} \mathrm{e}^{-\mathrm{i} \kappa^{\prime}\left(x^{\prime}+j d\right)} \mathrm{e}^{\mathrm{i} k \frac{\left(x^{\prime}+j d\right)^{2}}{2 r}} \psi\left(x^{\prime}, 0\right),
$$

which leads to a detection probability

$$
I_{\Omega}(\mathbf{r})=\int_{\vartheta-\frac{\Omega}{2}}^{\vartheta+\frac{\Omega}{2}} \mathrm{~d} \vartheta^{\prime} \frac{v}{4 \pi^{2} \lambda}\left|\sum_{j=0}^{N-1} \int_{-\frac{s}{2}}^{+\frac{s}{2}} \mathrm{~d} x^{\prime} \mathrm{e}^{-\mathrm{i} \kappa^{\prime} x^{\prime}} \mathrm{e}^{\mathrm{i} k \frac{\left(x^{\prime}+j d\right)^{2}}{2 r}} \psi\left(x^{\prime}, 0\right)\right|^{2}+\mathcal{O}\left(\frac{1}{r^{3}}\right) .
$$

This formula gives a description of particle scattering in the Fresnel limit of diffraction. It is valid as long as the detector position $\mathbf{r}$ and the size of the detector aperture $\Omega$ justify the use of straight trajectories within the eikonal approximation, i.e. the detector may not be further off-centre and the aperture not wider than a few rad.

If Eq. (2.69) is evaluated for a typical experimental set-up in which $r=0.43 \mathrm{~m}$ it becomes apparent as shown in Fig. 2.5 that due to the Fresnel corrections the shape of the principal diffraction maxima is quite unlike the idealized $\delta$-peak on which the practical formula Eq. (2.64) is based. Remarkably, it turns out at this point that Eq. (2.64) nevertheless can be used in good approximation. The reason for this very convenient fact is that although the principal maxima are considerably deformed by the Fresnel corrections they retain their character 


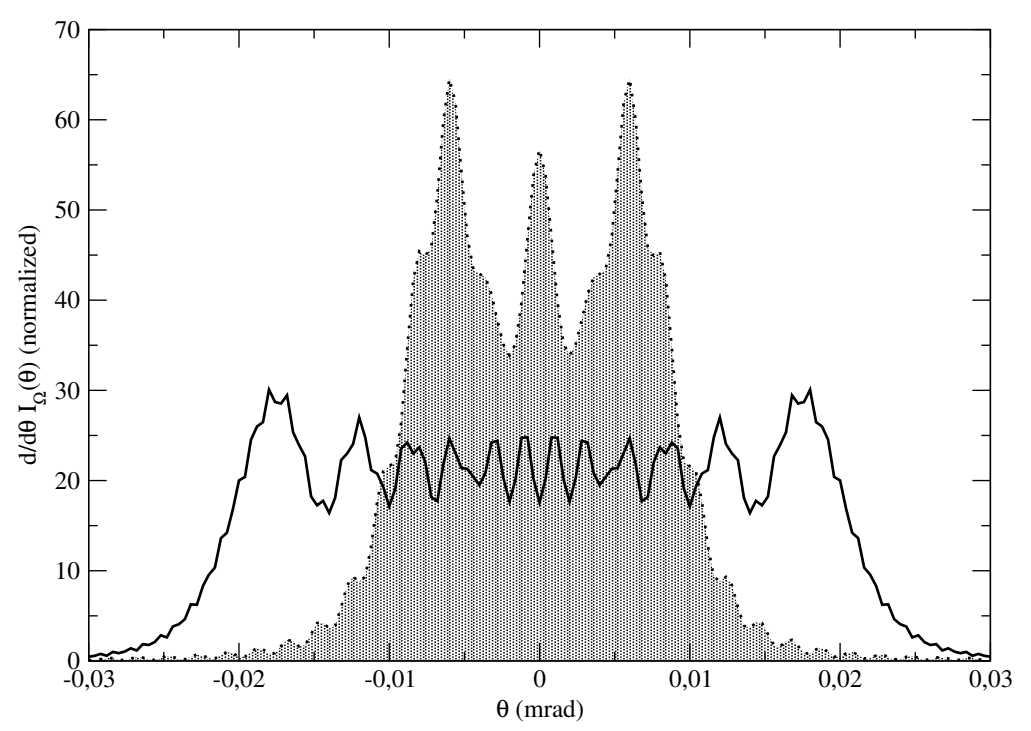

Figure 2.5: This figure is completely analogous to Fig. 2.4 except that Fresnel corrections are included with a typical grating-detector distance $r=0.43 \mathrm{~m}$. If the illuminated spot on the grating is $N=100$ slits wide (dotted line) the zeroth order peak is more narrow whereas for $N=200$ (solid line) the Fresnel corrections lead to a considerable broadening. The shaded area 0.9923 is practically equal to that 0.9917 under the solid curve, and both are sufficiently close to the area 1 that follows for the Fraunhofer limit with $N \rightarrow \infty$. Consequently, the simple formula Eq. (2.64), which is based on this limit, can be applied to the current experiments.

as $n$th diffraction orders in that the area under the peaks remains the same. Therefore, as long as neighbouring peaks in the diffraction patterns do not overlap (i.e. $\lambda$ not be too short) it is possible to describe the current experiments with the help of the Fraunhofer diffraction limit, as set out in the previous section, and to assume the number $N$ of illuminated slits to be very large so that the principal diffraction orders in theory become $\delta$ function-like. The theoretical peak shapes shown in Fig. 2.5 for monochromatic incident wavefunctions are not observed in the experiments because the distribution of angles and particle velocities in the incident beam and the detector aperture lead to a blurring and a broadening of the measured principal order maxima. As these are incoherent effects they do not interfere with the applicability of the Fraunhofer limit as recommended above. A more detailed discussion of this point is deferred until chapter 4 . 


\section{$2.7 \quad$ Summary}

The contents of this chapter are summarized as follows.

- The quantum mechanical scattering theoretical treatment of the scattering of atoms from the bars of a solid transmission grating results in a description that is largely analogous to the diffraction of light from a transmission grating whose depth is negligible.

- Aiming at an experimental situation with a high kinetic energy of the atomic beam and a small relevant range of scattering angles centred around zero one may neglect the reflected part of the scattered amplitude.

- What distinguishes the diffraction of atoms from the optical case is that on applying Huygens' principle one has to take account of an additional quantum mechanical phase which arises from the interaction of the transmitted atoms with the grating bars and which is calculated with the help of the distorted-wave Born approximation and the eikonal approximation.

- As a new result, it is observed that typical experiments actually fall in the regime of Fresnel diffraction theory but as long as they are based on the relative areas of well separated principal order peaks the results can practically be compared with theoretical values calculated in the Fraunhofer limit of diffraction. 


\section{Chapter 3}

\section{van der Waals Potentials}

In the literature, the term van der Waals (vdW) forces is widely employed to generally describe forces which act between electrically neutral objects such as atoms, molecules, or macroscopic bodies as a result of their electromagnetic polarization. This description of things subject to the vdW forces matches most of the matter of our daily encounter and so there is a number of everyday phenomena which have been correctly described in terms of these forces. Among these phenomena are the adhesion of smooth surfaces on each other, the physical adsorption of little particles on a surface, wetting effects and the surface tension of liquids. The behaviour of real gases, liquids, or thin films, in fact, is always a consequence of the van der Waals forces within. As a topic of current research, scientists are trying to figure out the way large molecules like polymer chains or proteins fold up as a result of the vdW forces between their constituent parts. The fundamental character of the van der Waals forces becomes apparent in the famous Casimir effect that leads to a non-gravitational attraction between two metal plates which are placed parallel at a mutual distance of centimetres in free space, and each of which possesses no static electromagnetic field.

From the observed strength of the vdW forces and their presence in the vacuum it is clear that they are of electromagnetic origin, ruling out gravitation and nuclear forces. As the interaction partners are electrically neutral the vdW forces must be due to higher multipoles of the charge distributions within the interacting objects. Quite generally, three cases can be distinguished,

1. the force between permanent multipoles, e.g. two polar molecules can be represented by two dipoles,

2. the force that arises when permanent multipoles induce a polarization within the other interaction partner,

3. the forces due to mutually induced polarization which results from everpresent charge density fluctuations within each of the interaction partners. 
While the first two aspects might be described by classical electrodynamics the third is of purely quantum mechanical origin. The problem of atoms interacting with the electrically neutral and unpolarized material of a transmission grating, which is a central topic in this work, is entirely due to the third of the cases enumerated. It is referred to by the names dispersion force, electrodynamic force, or charge fluctuation force; in the following the name dispersion force shall be adopted. The pioneering works on the dispersion force by London (1930) [38, 3.9], Casimir and Polder (1948) [40], and Lifshitz with co-workers (1956) [41] were focused on the interaction via mutually induced dipole moments so the common term London force is understood as to refer to this limit.

In the years after the pioneering works the dispersion force has been extensively investigated by many authors which has led to a generalization and also a simplification of the concepts involved. Today, the principles can be found in standard textbooks on quantum mechanics [42], whereas concrete applications are still a subject of current research.

To establish a connection between theoretically and experimentally determined values of the dispersion interaction strength between rare gas atoms and the material of the transmission gratings it is usual on the theoretical side to go back to the situation of an atom in front of an infinite half-space of the given material]. On the experimental side it has proven difficult to relate measured results obtained with macroscopic bodies of a certain shape to corresponding theoretical predictions. In a famous series of experiments between 1969 and 1975 Raskin, Kusch and co-workers determined the dispersion force by measuring the deflection of an atomic beam flying past a glass cylinder covered with a metal film [13, 43, 44, 45]. Their results repeatedly differed from theoretical predictions by some $50 \%$ which has sparked a number of subsequent publications by various authors [46, 47, 48, 4.9]. Finally, the discrepancy was ascribed to the crucial influence of unknown parameters such as surface roughness so that the experiment was actually found not suitable to be compared to any theory due to a lack of specified information [50, 51]].

Generally, the measured values of the interaction strength for a certain atom and a certain surface do not tend to coincide to better than about $30 \%$ [52, 53]. Theoretical calculations usually suffer from a lack of accurate optical data on the solid material which will be shown crucial for evaluating the interaction strength. The extrapolation of optical data is usually performed in the most simple oneelectronic-oscillator model for the solid which leads to an acknowledged uncertainty around $20 \%$ in the interaction strength [36, 55].

The first simple experimental method to measure the dispersion interaction strength was presented in 1999 by Grisenti et al. [ए2] who determined the potential between a beam of rare gas atoms and the bars of a (dielectric) silicon

\footnotetext{
${ }^{1}$ In fact, the interaction strength coefficient is defined in terms of this standard situation.
} 
nitride transmission grating by relating the diffracted intensity to the dispersion potential. A similar experiment with metastable rare gas atoms has recently been published [54] by our group and will be treated later on in this work. The following sections provide the theoretical concepts that will allow for the first time to calculate the relevant atom-surface interaction strengths, so that the old results for ground-state atoms and the new results for metastable atoms, both with silicon nitride gratings, can be compared to theoretical values. Due to recent progress in acquiring and extrapolating optical data for silicon nitride [97] the calculations exhibit a relatively high accuracy.

\subsection{The Dispersion Force}

The dispersion force is a quantum mechanical effect. Intuitively, it can be explained as arising from spontaneous and mutually induced charge fluctuations within the interaction partners. These fluctuations are expressed in terms of the frequency-dependent susceptibilities of the interacting objects to an electric field. The susceptibilities in turn are derived from the electronic degrees of freedom which are characteristic for the respective atoms, molecules, or solids. In particular, the force depends on the symmetry properties of all their electronic quantum states so that in general the interaction does not follow a simple single power law in terms of the mutual distance [56, 57, 58, 5.9, 60] ], it can also be anisotropic and repulsive or attractive under varying conditions [15, [6, 6]]. Especially the possibility of a repulsive dispersion interaction is a matter of current interest [62] as it involves applications for the growing industry of nanomachinery. The presence of a third interaction partner always changes the mutual response to charge fluctuations within the other two, hence the dispersion force is non-additive, unlike the pure Coulomb interaction [63, 64, 65] .

In any case, the dispersion force between objects is mediated by the electromagnetic field in between. Any response will thus be retarded by the time light takes to cover the distance between the interaction partners. This retardation is known to weaken the force, thereby adding to the difficult task of finding a closed expression for the interaction strength as a function of the distance. As a rule, the effect of retardation is said to be negligible if the distances between the objects involved are less than the wavelength $\lambda_{0}$ of a photon whose energy matches that of the most likely electronic transition within the system.

For the simple case of two ground-state atoms $\mathrm{A}$ and $\mathrm{B}$ at a distance $R \ll \lambda_{0}$, which allows retardation to be ignored, the dispersion potential $V(R)$ can be obtained from an electric multipole expansion that yields

$$
V(R)=-\frac{C_{6}}{R^{6}}-\frac{C_{8}}{R^{8}}-\frac{C_{10}}{R^{10}}-\ldots
$$


The leading dipole-dipole term can be derived from stationary perturbation theory with the Hamiltonian $H=H_{0}+V$, with $H_{0}|00\rangle=2 E_{0}|00\rangle$, and where

$$
V:=\frac{D}{R^{3}}:=\frac{e^{2}}{4 \pi \epsilon_{0} R^{3}}\left(x_{\mathrm{A}} x_{\mathrm{B}}+y_{\mathrm{A}} y_{\mathrm{B}}-2 z_{\mathrm{A}} z_{\mathrm{B}}\right)
$$

represents the energy of a dipole $e \mathbf{X}_{\mathrm{A}}$ in the field of another dipole $e \mathbf{X}_{\mathrm{B}}$ at a distance $R$. The ket $|00\rangle$ represents the ground-state of both atoms. While the first order energy correction vanishes the second order correction becomes

$$
\Delta E=-\frac{C_{6}}{R^{6}}
$$

with

$$
C_{6}=\sum_{n, m=1}^{\infty} \frac{|\langle 00|D| n m\rangle|^{2}}{\left(E_{0}-E_{n}\right)+\left(E_{0}-E_{m}\right)} .
$$

For the sake of simplicity the sum in Eq. (3.4) is restricted to the the most important contributions that arise from nearest excited states $|n m\rangle$ of the two atoms and that are taken to be a degenerate triplet of $p$-states like in hydrogen atoms. This yields

$$
C_{6}=\frac{6 e^{4}}{\left(4 \pi \epsilon_{0}\right)^{2} \hbar} \sum_{n, m>0} \frac{\left|\left\langle 0\left|x_{\mathrm{A}}\right| n\right\rangle\right|^{2}\left|\left\langle 0\left|x_{\mathrm{B}}\right| m\right\rangle\right|^{2}}{\omega_{n}+\omega_{m}},
$$

with $\hbar \omega_{i}:=E_{0}-E_{i}$. This result, first obtained by Eisenschitz and London in 1930 [38] can be rewritten using the identity [66]

$$
\frac{1}{\omega_{n}+\omega_{m}}=\frac{2}{\pi} \int_{0}^{\infty} \mathrm{d} \omega \frac{\omega_{n} \omega_{m}}{\left(\omega_{n}^{2}+\omega^{2}\right)\left(\omega_{m}^{2}+\omega^{2}\right)}
$$

It follows that

$$
C_{6}=\frac{3 \hbar}{\pi} \int_{0}^{\infty} \mathrm{d} \omega \alpha_{\mathrm{A}}(\mathrm{i} \omega) \alpha_{\mathrm{B}}(\mathrm{i} \omega)
$$

where

$$
\alpha_{i}(\xi):=\frac{e^{2}}{4 \pi \epsilon_{0}} \frac{2}{\hbar} \sum_{n>0} \frac{\left|\left\langle 0\left|x_{i}\right| n\right\rangle\right|^{2} \omega_{n}}{\omega_{n}^{2}-\xi^{2}} \quad, \quad i=\mathrm{A}, \mathrm{B}
$$

are the usual frequency-dependent atomic polarizabilities, that are evaluated in Eq. (3.7) at imaginary frequencies, which is accomplished by setting $\xi=\mathrm{i} \omega$ in Eq. (3.8). This is convenient because the polarizability $\alpha$ on the imaginary axis is a real monotonously decreasing function without poles. A comprehensive explanation of this general feature of susceptibilities, for which the atomic polarizability $\alpha(\omega)$ is a standard example, can be found in Landau and Lifshitz' textbook 
on statistical physics [67]. As a result, the dipole contribution to the dispersion interaction energy of two hydrogen atoms in their ground-state is given by

$$
\Delta E(R)=-\frac{1}{R^{6}} \frac{3 \hbar}{\pi} \int_{0}^{\infty} \mathrm{d} \omega \alpha^{2}(\mathrm{i} \omega) .
$$

In 1948 Casimir and Polder [40] have shown that in the retarded limit $R \gg \lambda_{0}$ the interaction potential like in Eq. (3.9) becomes

$$
\Delta E(R)=-\frac{1}{R^{7}} \frac{23 \hbar c \alpha^{2}(0)}{4 \pi},
$$

where $c$ stands for the speed of light, which is expected to appear in this equation for the retarded interaction. As a further detail, it may be noted that it is sufficient in this limit to use the static value $\alpha(0)$ of the polarizability. The reason for this will become clear later in this section.

In the years from 1948 to the early seventies several authors have presented their methods of developing the general expression from which both the nonretarded (Eisenschitz and London, Eq. (3.9)) and the retarded (Casimir and Polder, Eq. (3.10)) result can be derived. While the earlier attempts involved quite elaborate field theoretical calculations [68] that are beyond the scope of this text, some of the publications that followed explained the problem more intuitively by simplifying the required technique as far as possible. A well understandable approach has been presented by McLachlan in a series of papers beginning in $1962[63,6.9,60]$ that will serve to illustrate the underlying concepts on which the later calculations of the dispersion interaction strengths in this work are based.

McLachlan's approach starts from the notion that the interaction energy $\Delta E$ due to spontaneous charge fluctuations of an atom $\mathrm{A}$ at $\mathbf{r}_{\mathrm{A}}$, represented by an oscillating dipole, and the electromagnetic field around it is given by an integral over imaginary frequencies similar to Eq. (3.7), namely

$$
\Delta E=-\frac{\hbar}{2 \pi} \int_{0}^{\infty} \mathrm{d} \omega \alpha_{i k}^{\mathrm{A}}(\mathrm{i} \omega) E_{k i}\left(\mathbf{r}_{\mathrm{A}}, \mathbf{r}_{\mathrm{A}}, \mathrm{i} \omega\right)
$$

In this equation $\alpha_{i k}^{\mathrm{A}}$ stands for a component of the polarizability tensor $\underline{\boldsymbol{\alpha}}^{\mathrm{A}}$ of the atom $\mathrm{A}$, and $E_{k i}\left(\mathbf{r}, \mathbf{r}^{\prime}\right)$ in general is a component of the susceptibility tensor $\underline{\mathbf{E}}\left(\mathbf{r}, \mathbf{r}^{\prime}\right)$ of the electromagnetic field at $\mathbf{r}$ responding to an oscillating electric dipole at $\mathbf{r}^{\prime}$.

Here and in the following summation over repeated indices is implied. Both tensors are explicitly given by $3 \times 3$ matrices and are represented here by underscored bold types while their components are denoted by doubly indexed plain types. 
The components of the field susceptibility $E_{k i}\left(\mathbf{r}_{\mathrm{A}}, \mathbf{r}_{\mathrm{B}}, \mathrm{i} \omega\right)$ at $\mathbf{r}_{\mathrm{A}}$ as arising from an oscillating dipole at $\mathbf{r}_{\mathrm{B}}$ can be calculated classically via the spectral Fourier component $\mathbf{E}\left(\mathbf{r}_{\mathrm{A}}, \omega\right)$ of the electric field at $\mathbf{r}_{\mathrm{A}}$ that is generated by an oscillating dipole $\mathbf{P}^{\mathrm{B}}(\omega)$ located at $\mathbf{r}_{\mathrm{B}}$. Simply put, one can infer the field susceptibility if one knows the cause of the field (the dipole at $\mathbf{r}_{\mathrm{B}}$ ) and the field itself at $\mathbf{r}_{\mathrm{A}}$ because the susceptibility is by definition just the quantity that connects the two. The formal expression of the above said is

$$
\mathbf{E}\left(\mathbf{r}_{\mathrm{A}}, \omega\right)=\underline{\mathbf{E}}\left(\mathbf{r}_{\mathrm{A}}, \mathbf{r}_{\mathrm{B}}, \omega\right) \mathbf{P}^{\mathrm{B}}(\omega) .
$$

The calculation of the field $\mathbf{E}\left(\mathbf{r}_{\mathrm{A}}, \omega\right)$ follows a standard procedure starting from the electromagnetic wave equation with the electric dipole as an inhomogeneity which yields

$$
\mathbf{E}\left(\mathbf{r}_{\mathrm{A}}, \omega\right)=\frac{1}{4 \pi \epsilon_{0}} \boldsymbol{\nabla} \times\left(\boldsymbol{\nabla} \times \frac{\mathrm{e}^{\frac{\mathrm{i} \omega R}{c}}}{R} \mathbf{P}^{\mathrm{B}}(\omega)\right)
$$

with $\mathbf{R}:=\mathbf{r}_{\mathrm{A}}-\mathbf{r}_{\mathrm{B}}$. Without restricting generality the $z$ axis of the coordinate frame is chosen parallel to $\mathbf{R}$ and an explicit evaluation of Eq. (3.13) yields

$$
\mathbf{E}\left(\mathbf{r}_{\mathrm{A}}, \omega\right)=\frac{\mathrm{e}^{\mathrm{i} R k}}{4 \pi \epsilon_{0} R^{3}}\left(\begin{array}{ccc}
\mathrm{i} R k-1+R^{2} k^{2} & 0 & 0 \\
0 & \mathrm{i} R k-1+R^{2} k^{2} & 0 \\
0 & 0 & 2(1-\mathrm{i} R k)
\end{array}\right) \mathbf{P}^{\mathrm{B}}(\omega)
$$

with $k:=\frac{\omega}{c}$. The field susceptibility tensor is identified by comparison of this result with Eq. (3.12). Like in Eq. (3.8), it is in accordance with the analytical properties of the susceptibility to change to imaginary frequencies by simply replacing $\omega \mapsto \mathrm{i} \omega$.

With the field susceptibility thus available it is important to note that by the way it has been put Eq. (3.11) represents the infinite interaction energy of the atom A with its own dipole field, because $R=\left|\mathbf{r}_{\mathrm{A}}-\mathbf{r}_{\mathrm{A}}\right|=0$ so that Eq. (3.14) diverges. There is no physical meaning associated with this but if another atom $B \neq A$ is present the concept can be interpreted to make sense. Through its dipole moment $\mathbf{P}^{\mathrm{B}}$ the atom $\mathrm{B}$ adds to the field susceptibility around atom $\mathrm{A}$ a finite term so that the "difference" $[$ of interaction energies of atom A with the field for the cases with or without the second atom B is finite and is identified with the mutual dispersion energy of the two atoms $\mathrm{A}$ and $\mathrm{B}$.

The formal expression of this is to replace in Eq. (3.11) the field tensor $E_{k i}\left(\mathbf{r}_{\mathrm{A}}, \mathbf{r}_{\mathrm{A}}, \mathrm{i} \omega\right)$ by a modified one $\hat{E}_{k i}\left(\mathbf{r}_{\mathrm{A}}, \mathbf{r}_{\mathrm{A}}, \mathrm{i} \omega\right)$ that describes the interaction of atom $\mathrm{A}$ as carried over to atom $\mathrm{B}$ by the electromagnetic field and then back to A.

\footnotetext{
${ }^{2}$ Infinite plus finite minus infinite is not a proper difference. Keeping in this as a result the finite term is called a renormalization, which is common in quantum electrodynamics.
} 
The modified field susceptibility $\hat{E}_{k i}\left(\mathbf{r}_{\mathrm{A}}, \mathbf{r}_{\mathrm{A}}, \mathrm{i} \omega\right)$ is obtained as follows. In Eq. (3.12), one interprets the dipole moment $\mathbf{P}^{\mathrm{B}}(\omega)$ as the result of atom $\mathrm{B}$ responding to the electric field

$$
\mathbf{E}\left(\mathbf{r}_{\mathrm{B}}, \omega\right)=\underline{\mathbf{E}}\left(\mathbf{r}_{\mathrm{B}}, \mathbf{r}_{\mathrm{A}}, \omega\right) \mathbf{P}^{\mathrm{A}}(\omega) .
$$

generated by the oscillating dipole of atom A. The response of atom B is then given by

$$
\mathbf{P}^{\mathrm{B}}(\omega)=\underline{\boldsymbol{\alpha}}^{\mathrm{B}}(\omega) \mathbf{E}\left(\mathbf{r}_{\mathrm{B}}, \omega\right),
$$

which is where the polarizability tensor $\underline{\boldsymbol{\alpha}}^{\mathrm{B}}(\omega)$ of atom B necessarily enters.

Combining Eq. (3.15) and Eq. (3.16) one has

$$
\mathbf{P}^{\mathrm{B}}(\omega)=\underline{\boldsymbol{\alpha}}^{\mathrm{B}}(\omega) \underline{\mathbf{E}}\left(\mathbf{r}_{\mathrm{B}}, \mathbf{r}_{\mathrm{A}}, \omega\right) \mathbf{P}^{\mathrm{A}}(\omega)
$$

It is now possible to obtain an equation that is formally analogous to Eq. (3.12) and thus similarly allows to calculate the modified field susceptibility $\hat{E}_{k i}\left(\mathbf{r}_{\mathrm{A}}, \mathbf{r}_{\mathrm{A}}, \mathrm{i} \omega\right)$. For this purpose one inserts the right-hand-side of Eq. (3.17) in Eq. (3.12) which yields

$$
\mathbf{E}\left(\mathbf{r}_{\mathrm{A}}, \omega\right)=\underline{\hat{\mathbf{E}}}\left(\mathbf{r}_{\mathrm{A}}, \mathbf{r}_{\mathrm{A}}, \omega\right) \mathbf{P}^{\mathrm{A}}(\omega),
$$

with the matrix product

$$
\underline{\hat{\mathbf{E}}}\left(\mathbf{r}_{\mathrm{A}}, \mathbf{r}_{\mathrm{A}}, \omega\right)=\underline{\mathbf{E}}\left(\mathbf{r}_{\mathrm{A}}, \mathbf{r}_{\mathrm{B}}, \omega\right) \underline{\boldsymbol{\alpha}}^{\mathrm{B}}(\omega) \underline{\mathbf{E}}\left(\mathbf{r}_{\mathrm{B}}, \mathbf{r}_{\mathrm{A}}, \omega\right) .
$$

After changing to imaginary frequencies the interaction energy between the atoms $\mathrm{A}$ and $\mathrm{B}$ becomes

$$
\Delta E(R)=-\frac{\hbar}{2 \pi} \int_{0}^{\infty} \mathrm{d} \omega \alpha_{i k}^{\mathrm{A}}(\mathrm{i} \omega) \hat{E}_{k i}\left(\mathbf{r}_{\mathrm{A}}, \mathbf{r}_{\mathrm{A}}, \mathrm{i} \omega\right)
$$

where the $R$ dependence is contained in $\hat{E}_{k i}$.

Within the approximation of isotropic atomic polarizabilities the tensors $\underline{\boldsymbol{\alpha}}(\mathrm{i} \omega)$ become scalars $\alpha(\mathrm{i} \omega)$ and an explicit evaluation of Eq. (3.20) using Eq. (3.19) yields

$$
\Delta E(R)=-\frac{\hbar}{2 \pi R^{6}} \int_{0}^{\infty} \mathrm{d} \omega \alpha^{\mathrm{A}}(\mathrm{i} \omega) \alpha^{\mathrm{B}}(\mathrm{i} \omega) \mathrm{e}^{-2 R k}\left(6+12 k+10 k^{2}+4 k^{3}+2 k^{4}\right)
$$

In the limit of small $R$ London's result Eq. (3.9) is recovered by setting the exponential equal to 1 and by neglecting all terms of higher than zeroth order in $k R$. In the retarded limit $R$ is large and only small $k \equiv \frac{\omega}{c}$ significantly contribute to 
the integral. Therefore approximating $\alpha(\mathrm{i} \omega) \approx \alpha(0)$ and substituting $\xi:=R k$ it follows for large $R$

$$
\begin{aligned}
\Delta E(R) & =-\frac{\hbar c \alpha^{\mathrm{A}}(0) \alpha^{\mathrm{B}}(0)}{4 \pi R^{7}} \int_{0}^{\infty} \mathrm{d} \xi \mathrm{e}^{-\xi}\left(6+6 \xi+\frac{5 \xi^{2}}{2}+\frac{\xi^{3}}{2}+8 \xi^{4}\right)= \\
& =-\frac{23 \hbar c \alpha^{\mathrm{A}}(0) \alpha^{\mathrm{B}}(0)}{4 \pi R^{7}}
\end{aligned}
$$

which is Casimir and Polder's result Eq. (3.10).

The essence of the last few pages is thus Eq. (3.21) which is the "general formula" spoken of earlier from which both the non-retarded and the retarded limit of the dispersion interaction between two atoms can be derived.

\subsection{Atom-Solid Interaction}

In view of the experiments to be described later in this work, where atoms interact with the dielectric material of a transmission grating it is important for the study of retardation effects to obtain a formula similar to Eq. (3.21) for atoms interacting with a dielectric solid.

Speaking in terms of the concept explained in the previous section, the relevant finite part of the electric field susceptibility around the atom arises from charge fluctuations within the solid that are induced by the charge fluctuations within the atom. There is also another contribution that takes account of the fact that excitations of the electromagnetic field are reflected at the interface vacuum-dielectric. The reflection behaviour is usually described in terms of Fresnel coefficients which depend on the dielectric properties of the media involved and on the polarization of the field quanta [71]. In reality, the (heterogeneous) composition and (corrugated) structure of the surface will also influence the reflectivity.

For a perfect surface and reflection included Lifshitz has shown in 1956 how a formula for the interaction energy between single atoms and a dielectric surface at all distances can be derived from a general theory [4T]. It turns out that the radiation scattered at the interface is most relevant for large distances, in the retarded regime. For closer distances an atom A represented by an oscillating dipole of spectral density $\mathbf{P}^{\mathrm{A}}(\omega)$ and located at $\mathbf{r}_{\mathrm{A}}=r \hat{\mathbf{e}}_{z}$ opposite a dielectric halfspace defined by $z<0$ can be described in terms of an image dipole $\frac{\epsilon(\omega)-1}{\epsilon(\omega)+1} \mathbf{P}^{\mathrm{B}}(\omega)$ at $-\mathbf{r}_{\mathrm{A}}$ within the solid whose dielectric function is $\epsilon(\omega)$. This approximation assumes that the dielectric response of the solid is isotropic, and it neglects the effects associated with the reflected radiation but includes retardation. It is expected to be correct for distances of the order of, or slightly larger than the 
"retardation limit" $\lambda_{0}$. Within this approximation the interaction energy can be written in a form similar to Eq. (3.20) as

$$
\Delta E(R)=-\frac{\hbar}{2 \pi} \int_{0}^{\infty} \mathrm{d} \omega \alpha_{i k}^{\mathrm{A}}(\mathrm{i} \omega) E_{k i}^{\mathrm{diel}}\left(\mathbf{r}_{\mathrm{A}}, \mathbf{r}_{\mathrm{A}}, \mathrm{i} \omega\right)
$$

with the atom-dielectric field susceptibility tensor

$$
\underline{\mathbf{E}}^{\text {diel }}\left(\mathbf{r}_{\mathrm{A}}, \mathbf{r}_{\mathrm{A}}, \omega\right)=\frac{\mathrm{e}^{\mathrm{i} \xi}}{4 \pi \epsilon_{0} R^{3}}\left(\begin{array}{ccc}
-\left(\mathrm{i} \xi-1+\xi^{2}\right) & 0 & 0 \\
0 & -\left(\mathrm{i} \xi-1+\xi^{2}\right) & 0 \\
0 & 0 & 2(1-\mathrm{i} \xi)
\end{array}\right),
$$

with $\xi \equiv R k$ and $k \equiv \frac{\omega}{c}$. Note that the minus sign of the $x x$ and $y y$ components accounts for the inverted orientation of the mirror dipole. Expressed in terms of the atom-surface distance $l=\frac{R}{2}$ and for a scalar atomic polarizability the interaction energy becomes

$$
\Delta E(l)=-\frac{\hbar}{4 \pi l^{3}} \int_{0}^{\infty} \mathrm{d} \omega \alpha^{\mathrm{A}}(\mathrm{i} \omega) g(\mathrm{i} \omega) \mathrm{e}^{-2 k l}\left(1+k l+k^{2} l^{2}\right),
$$

where the electronic response $g(\mathrm{i} \omega)$ of the solid is defined by

$$
g(\mathrm{i} \omega):=\frac{\epsilon(\mathrm{i} \omega)-1}{\epsilon(\mathrm{i} \omega)+1} .
$$

The non-retarded limit of Eq. (3.25) for the dispersion potential between an atom and a dielectric surface is widely used, especially for adsorption problems. It is obtained by taking $l$ small enough to replace the exponential by unity and neglect all terms higher than zeroth order in $k l$. This yields

$$
\Delta E(l)=-\frac{C_{3}}{l^{3}}
$$

with the interaction strength $C_{3}$ given by the so-called Lifshitz formula

$$
C_{3}=\frac{\hbar}{4 \pi} \int_{0}^{\infty} \mathrm{d} \omega \alpha^{\mathrm{A}}(\mathrm{i} \omega) g(\mathrm{i} \omega)
$$

It is clear from Eq. (3.25) that in the general case the dispersion interaction strength $C_{3}$ depends on the atom-surface distance $l$, in particular, it is reduced due to retardation for increasing $l$. A quantitative discussion of this point is deferred until section 4.4 of this text. 
For the non-retarded limit the formulae Eq. (3.27) and Eq. (3.28) show that in order to calculate $\Delta E(l)$ at any distance $l$ a complete knowledge of the atomic polarizability $\alpha(\mathrm{i} \omega)$ and the electronic response $g(\mathrm{i} \omega)$ of the solid is required. The corresponding complex frequency-dependent susceptibilities, respectively, $\alpha(\omega) \equiv$ $\alpha^{\prime}(\omega)+\mathrm{i} \alpha^{\prime \prime}(\omega)$ and $g(\omega) \equiv g^{\prime}(\omega)+\mathrm{i} g^{\prime \prime}(\omega)$ are known from general considerations of their analytic properties [67] to be real, continuous, monotonically decreasing functions on the positive imaginary axis. Their values on the positive imaginary axis are connected with those of their imaginary parts at real frequencies through Kramers-Kronig relations of the form

$$
\alpha(\mathrm{i} \omega)=\frac{2}{\pi} \int_{0}^{\infty} \mathrm{d} \xi \frac{\alpha^{\prime \prime}(\xi) \xi}{\omega^{2}+\xi^{2}}
$$

for the atom and

$$
\epsilon(\mathrm{i} \omega)=1+\frac{2}{\pi} \int_{0}^{\infty} \mathrm{d} \xi \frac{\epsilon^{\prime \prime}(\xi) \xi}{\omega^{2}+\xi^{2}},
$$

through the definition Eq. (3.26) of $g(\mathrm{i} \omega)$ for the solid. The following two sections present a semi-empirical way to find the dynamic atomic polarizability $\alpha(\mathrm{i} \omega)$ at imaginary frequencies, and the response $g(\mathrm{i} \omega)$ of the solid. The result will in principal allow, for an arbitrary choice of atoms and dielectrics, to evaluate Eq. (3.25) and especially the Lifshitz formula Eq. (3.28) and so obtain theoretical values of the dispersion interaction strength $C_{3}$. In view of later applications in this work a focus is put on rare-gas atoms and the amorphous silicon nitride material of the transmission gratings.

\subsection{Atomic Polarizability}

For the atom, the easiest approach to the polarizability is via Eq. (3.8). It is today a common procedure [72] to construct the eigenstates of light atomic species and evaluate the matrix elements in Eq. (3.8) to directly obtain $\alpha(\mathrm{i} \omega)$ by

$$
\alpha(\mathrm{i} \omega):=\frac{e^{2}}{4 \pi \epsilon_{0}} \frac{2}{\hbar} \sum_{n} \frac{|\langle 0|\mathbf{X}| n\rangle|^{2} \omega_{n}}{\omega_{n}^{2}+\omega^{2}}
$$

The empirical way of dealing with the last equation is to write

$$
\alpha(\mathrm{i} \omega)=\frac{e^{2}}{\left(4 \pi \epsilon_{0}\right)^{2} m} \sum_{n} \frac{f_{n}}{\omega_{n}^{2}+\omega^{2}}
$$

and fit the oscillator strengths $f_{n}$ and the transition frequencies $\omega_{n}$ to values obtained from gas spectroscopy. Today, experimental and theoretical data for 
the frequency-dependent polarizability of ground-state rare-gas and other atoms are in very good agreement [73, 74, 75, [76]. Since lately accurate theoretical calculations of the frequency-dependent polarizability have also been performed for the lowest-lying metastable states of rare-gas atoms [[77, [78, [79, 801, 81, 82, 8:3]. Although the dispersion interaction of these excited atoms requires a special treatment, as from fundamental considerations of the van der Waals interaction, the lifetimes involved are all long enough to treat the metastable states like "ground-states" from which no downward transitions are likely to occur.

With $\alpha(\mathrm{i} \omega)$ given theoretically or semi-empirically it is by Eq. (3.7) straightforward to determine the mutual dispersion interaction strength $C_{6}$ of two identical rare-gas atoms. Table 3.1 displays accurately calculated values of $C_{6}$ for ground-state and metastable rare-gas atoms as taken from the literature.

It has been noted by several authors that in order to determine the mutual interaction coefficients $C_{6}$ for different atomic species it is correct within a few percent to represent the dynamic atomic polarizabilities as due to single oscillators at characteristic electronic transition frequencies $\omega_{\mathrm{a}}$ for each atom by

$$
\alpha(\mathrm{i} \omega)=\frac{\alpha_{0}}{1+\frac{\omega^{2}}{\omega_{a}^{2}}} .
$$

Note hereby that through the Kramers-Kronig relations

$$
\lim _{\omega \rightarrow 0} \alpha^{\prime}(\omega)=\lim _{\omega \rightarrow 0} \frac{2}{\pi} \int_{0}^{\infty} \mathrm{d} \xi \frac{\alpha^{\prime \prime}(\xi) \xi}{\xi^{2}-\omega^{2}}=\frac{2}{\pi} \int_{0}^{\infty} \mathrm{d} \xi \frac{\alpha^{\prime \prime}(\xi)}{\xi} \stackrel{\text { Eq. }}{=} \lim _{\omega \rightarrow 0} \alpha(\mathrm{i} \omega) \equiv \alpha_{0}
$$

Eq. (3.33) is correct in the static limit by definition. For both ground-state and metastable rare-gas atoms the respective values of the static polarizabilities $\alpha(0) \equiv \alpha_{0}$ are well established and can be found in standard literature. Relevant values are on display in Table 3.1.

For reasons of consistency the characteristic atomic excitation frequency $\omega_{\mathrm{a}}$ of each species is taken such as to reproduce the interaction constant $C_{6}$ that appears on the left-hand-side of Eq. (3.7). Inserting the one-oscillator approximation Eq. (3.33) into Eq. (3.7) for two like atoms yields

$$
C_{6}=\frac{3 \hbar}{\pi} \int_{0}^{\infty} \mathrm{d} \omega \frac{\alpha_{0}^{2}}{\left(1+\frac{\omega^{2}}{\omega_{\mathrm{a}}^{2}}\right)^{2}}=\frac{3 \alpha_{0}^{2} \hbar \omega_{\mathrm{a}}}{4}
$$

from which follows

$$
E_{\mathrm{a}}:=\hbar \omega_{\mathrm{a}}=\frac{4 C_{6}}{3 \alpha_{0}^{2}} .
$$

The resulting characteristic energies $E_{\mathrm{a}}$ for atoms dealt with in this work are also listed in Table 3.1. In some cases (e.g. He, Ne; cf. Table 3.1) the characteris- 


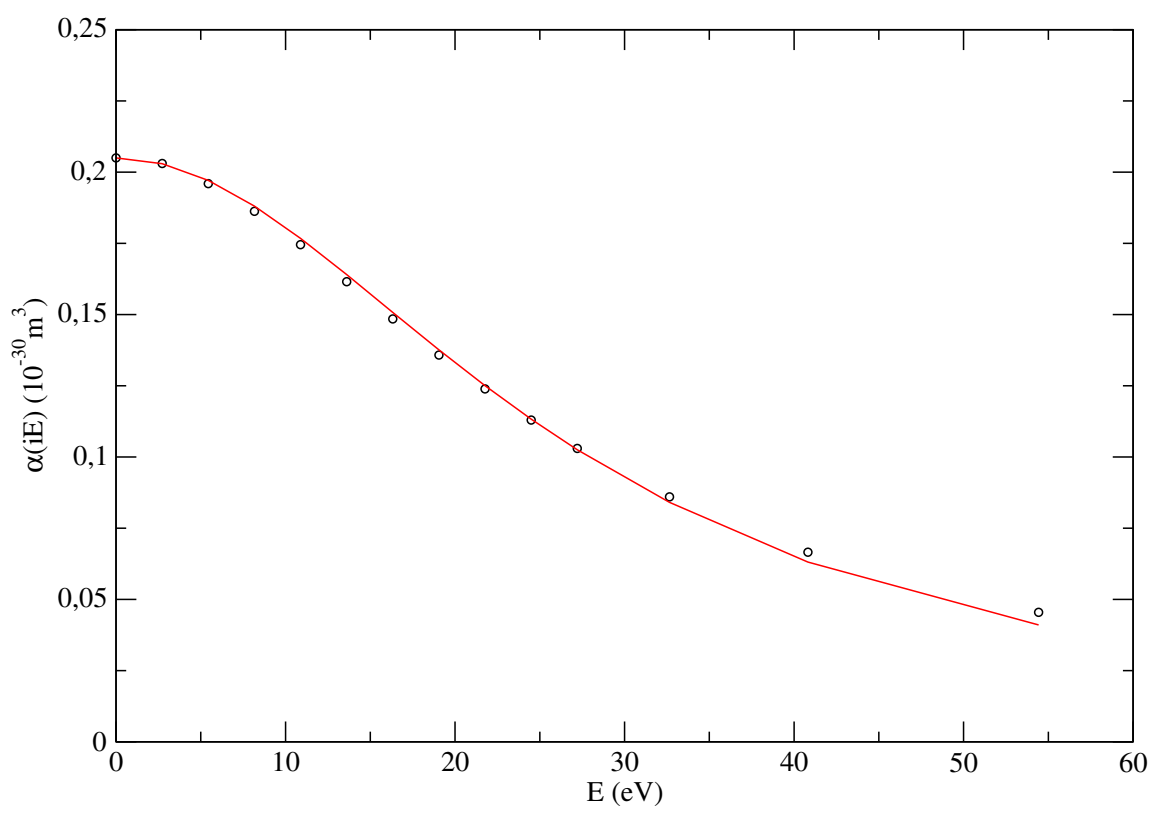

Figure 3.1: Precisely calculated values [76] of the dynamic polarizability $\alpha(\mathrm{i} E)$ of ground-state He atoms at imaginary energies $\mathrm{i} E$ (circles) are well fitted by the oneoscillator approximation Eq. (3.32) (solid line). $\alpha_{0}$ is taken as the static limit of the calculated data (see Eq. (3.33) $)$ while the best-fitted characteristic excitation energy $E_{\text {a }}$ of helium is $(27.2 \pm 0.7) \mathrm{eV}$, in agreement with the tabulated value (Table 3.1) $27.67 \mathrm{eV}$. The fitted curve displays systematic deviations at intermediate energies where it is too large and at large energies where it is too small (see text).

\begin{tabular}{c||c|c|c|c|c|c|c|c|c} 
atom & $\mathrm{He}$ & $\mathrm{Ne}$ & $\mathrm{D}_{2}$ & $\mathrm{Ar}$ & $\mathrm{Kr}$ & $\mathrm{Ne}^{*}$ & $\mathrm{He}^{*}$ & $\mathrm{Ar}^{*}$ & $\mathrm{Kr}^{*}$ \\
\hline \hline $4 \pi \epsilon_{0} \alpha_{0}$ & 0.205 & 0.397 & 0.806 & 1.64 & 2.48 & 27.6 & 46.8 & 47.9 & 50.6 \\
$C_{6}$ & $0.872^{\mathrm{d}}$ & 3.764 & $7.23^{\mathrm{a}}$ & 38.83 & 77.67 & $1156.9^{\mathrm{b}}$ & $1957.6^{\mathrm{c}}$ & $2770.1^{\mathrm{b}}$ & $3165.6^{\mathrm{b}}$ \\
$E_{\mathrm{a}}$ & 27.67 & 31.84 & 14.84 & 19.25 & 16.84 & 2.025 & 1.192 & 1.610 & 1.649
\end{tabular}

Table 3.1: Atomic data for ground-state and metastable species. First row: Static polarizability $\left(\AA^{3}\right)$ from Ref. [94]. Second row: Dispersion interaction strength of two like particles $\left(\mathrm{eV} \AA^{6}\right)$ from Ref. [6i]]. Third row: Characteristic atomic excitation energy $(\mathrm{eV})$ as calculated with Eq. (3.36). The metastable states are ${ }^{3} \mathrm{P}_{2}$ except for $\mathrm{He}^{*}$ that is ${ }^{3} \mathrm{~S}_{1}$. Special references: ${ }^{\mathrm{a}}$ : Value for $\mathrm{H}_{2}$ adopted from [9.5]; ${ }^{\mathrm{b}}$ : Ref. [8.3]; ${ }^{\mathrm{c}}$ : Ref. [8.2]; d : Ref. [96]

tic atomic excitation energy $E_{\mathrm{a}}$ comes out higher than the respective ionization potential, which is ascribed to a finite electronic transition probability into continuum states [66].

A direct fit of the one-oscillator approximation Eq. (3.32) to diligently calculated values for the dynamic polarizability at imaginary energies of ground-state 
helium atoms [76] is shown in Fig. 3.1. The fitted $E_{\mathrm{a}}=27.2251 \mathrm{eV}$ reproduces the tabulated value (Table 3.1) $27.67 \mathrm{eV}$ within $2 \%$. K. T. Tang has pointed out that the systematic deviations of this approximation tend to cancel when two functions of the approximated type are multiplied and integrated over [84]. Therefore the one-oscillator approximation is especially suited for the purpose of calculating dispersion coefficients $C_{6}$ of two atoms or $C_{3}$ of an atom and a surface, the latter of which is important for this work and which is shown in the following sections to arise from integrals closely analogous to those just discussed.

\subsection{Response of the Solid}

The second step towards calculating the atom-solid dispersion coefficient $C_{3}$ is to determine the response $g(\mathrm{i} \omega)$ of the solid. Using the Kramers-Kronig relation Eq. (3.29) one has

$$
g(\mathrm{i} \omega)=\frac{2}{\pi} \int_{0}^{\infty} \mathrm{d} \xi \frac{\epsilon^{\prime \prime}(\xi) \xi}{\omega^{2}+\xi^{2}}\left[2+\frac{2}{\pi} \int_{0}^{\infty} \mathrm{d} \xi \frac{\epsilon^{\prime \prime}(\xi) \xi}{\omega^{2}+\xi^{2}}\right]^{-1} .
$$

The material of interest in this work is amorphous silicon nitride $\left(\mathrm{SiN}_{x}\right)$ because the transmission gratings used in the new atom diffraction experiments are etched out of an approximately $100 \mathrm{~nm}$ thick layer of $\mathrm{SiN}_{x}$. Amorphous silicon nitride is an important compound in the field of microelectronics where it is used as an anti-reflection coating in lithographic processes. By varying the manufacturing method and the parameters involved it is possible to make $\operatorname{SiN}_{x}$ films of welldefined properties. In particular, the the optical band gap width is a function of the stoichiometric coefficient $x$, which is controlled by the relative amount of gases from which the films are deposited [86]. An important step in the manufacturing process is to check the band gap by measuring the frequency-dependent index of refraction $n(\omega)$ and the absorption coefficient $\kappa(\omega)$. These are connected with the complex dielectric function $\epsilon(\omega)$ by the well-known formulae

$$
\epsilon^{\prime}(\omega)=n^{2}(\omega)-\kappa^{2}(\omega)
$$

and

$$
\epsilon^{\prime \prime}(\omega)=2 n(\omega) \kappa(\omega) .
$$

The transmission gratings for the atom diffraction experiments to be discussed in this work have been made by Savas and co-workers [2] at the MIT using a process called low pressure chemical vapour deposition (LPCVD). The authors have provided a measurement [85] of the optical data $n(\omega)$ and $\kappa(\omega)$ in the range between $1 \mathrm{eV}$ and $6 \mathrm{eV}$ for silicon nitride films from which the transmission gratings are made. A plot of the data is shown in Fig. 3.2. With Eq. (3.38) it is possible to 

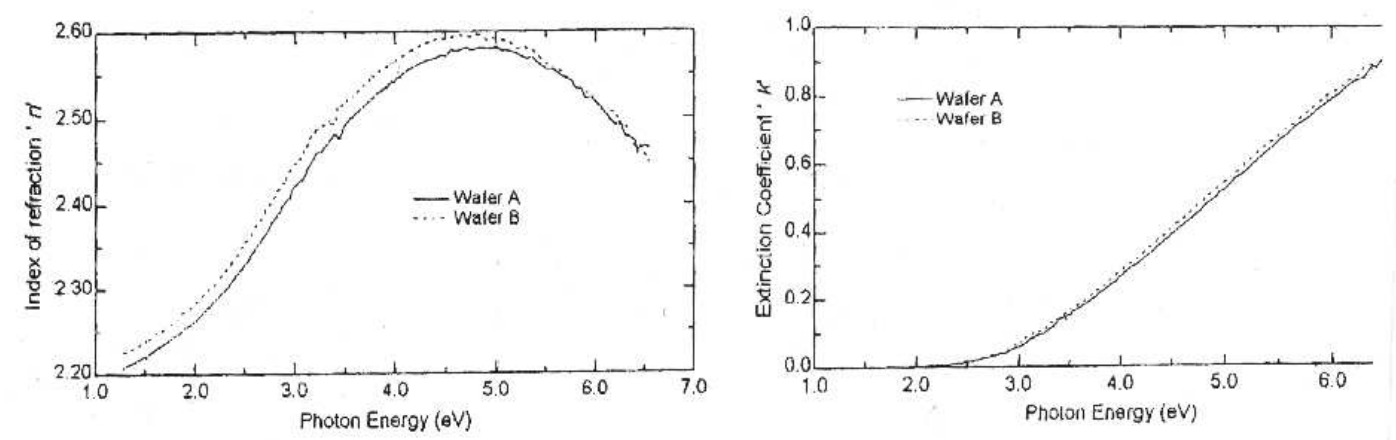

Figure 3.2: Energy-dependent refractive index $n(E)$ and absorption coefficient $\kappa(E)$ for two silicon nitride samples as communicated by Savas [85]. The onset of $\kappa(E)$ at about $2.2 \mathrm{eV}$ marks the width of the optical band gap $\Delta$ inside the amorphous material. The static value of $n(E)$ as arising from electronic excitations in the solid can be extrapolated as $n(0) \approx 2.1$. The plot of $\kappa(E)$ is cut off on the right as on the original communication.

extract the imaginary part of the dielectric function $\epsilon^{\prime \prime}(\omega)$ in the given frequency range. However, in order to determine the dispersion coefficient $C_{3}$ via Eq. (3.28) one needs $\epsilon(\mathrm{i} \omega)$ at imaginary frequencies and this requires by the Kramers-Kronig relation Eq. (3.30) the knowledge of $\epsilon^{\prime \prime}(\omega)$ over the entire frequency range from zero to infinity.

In the past, people proceeded by fitting $\epsilon^{\prime \prime}(\omega)$ in the limited range with an ansatz that included one or more electronic oscillators representing the solid and so were able to extrapolate $\epsilon^{\prime \prime}(\omega)$ to high frequencies. While more-oscillator solutions proved difficult to handle because of the large number of parameters, the one-oscillator approach, similar to that described above for atoms, lacks some accuracy. The crucial point is that a simple oscillator model does not account for the optical band gap $\Delta$, i.e. a range of frequencies $0<\omega<\Delta$ where no electronic transitions are likely to occur within the solid. This leads to errors in the calculation of $C_{3}$ Eq. (3.28) if the characteristic atomic transition frequency falls in the range of the optical band gap of the solid as is the case for metastable rare-gas atoms and silicon nitride (cf. Table 3.1).

In a recent publication Zollner and Apen of the Motorola company have studied the optical properties of LPCVD-made amorphous silicon nitride layers in detail. They point out that the imaginary part $\epsilon^{\prime \prime}(\omega)$ of the dielectric function is well parameterized by the Tauc-Lorentz formula [97]

$$
\epsilon^{\prime \prime}(\omega)=\Theta\left(\omega-\Omega_{\mathrm{T}}\right) \frac{A \Omega \Gamma\left(\omega-\Omega_{\mathrm{T}}\right)^{2}}{\left[\left(\omega^{2}-\Omega^{2}\right)^{2}+\Gamma^{2} \omega^{2}\right] \omega},
$$

where the optical band gap appears explicitly as $\Delta \equiv \hbar \Omega_{\mathrm{T}}$. $\Theta$ is the step func- 
tion and $A, \Omega, \Gamma$ are the strength, frequency, and spectral width, respectively, of one characteristic electronic transition within the solid. The measurement of refractive index $n(\omega)$ and the absorption coefficient $\kappa(\omega)$ communicated by Savas [85] bears a convincing resemblance to that published by Zollner and Apen for LPCVD anti-reflection coatings, while it differs from those for silicon nitride made by another technique in terms of the magnitude of $n$ and the onset of $\kappa$ which marks the width of the optical band gap. By comparison with other sources in the literature $[87,88,8.9,90,90]$ it turns out that the latter two features can in fact be used to identify the manufacturing process by looking at the optical data in the given range. As a result, it is plausible that the measurement of Savas has been performed on a LPCVD silicon nitride sample similar to the anti-reflection coatings tested by Zollner and Apen. Savas asserts that the measured material is identical with that of the transmission gratings that have been used in the diffraction experiments to be discussed.

From the foregoing observations it is concluded that the best way to extrapolate $\epsilon^{\prime \prime}(\omega)$ for the transmission gratings is to use the Tauc-Lorentz formula Eq. (3.40). It is expected to be more correct for low frequencies in the range of the optical band gap, while in the limit of large frequencies it is comparable to usual extrapolation methods. On extracting $\epsilon^{\prime \prime}(\omega)$ via Eq. (3.38) from Savas' data and fitting to it the Tauc-Lorentz formula Eq. (3.40) one obtains good agreement as is illustrated in Fig 3.3.

By inserting the fitted $\epsilon^{\prime \prime}(\omega)$ into Eq. (3.37) and subsequent numerical integration the electronic response $g(\mathrm{i} \omega)$ of silicon nitride is obtained. Combining this result with the atomic polarizability as in Eq. (3.33), finally, the atom-SiN ${ }_{x}$ dispersion coefficient $C_{3}$ is determined by numerical integration of Eq. (3.28). Table 3.2 shows the appropriate values for ground-state and metastable rare-gas atoms. The deuterium molecule $D_{2}$ that also appears in the table is hereby and in the following counted among the ground-state atoms because due to its small size it can be treated like an atom in the present diffraction experiments.

\begin{tabular}{c||c|c|c|c|c|c|c|c|c} 
atom & $\mathrm{He}$ & $\mathrm{Ne}$ & $\mathrm{D}_{2}$ & $\mathrm{Ar}$ & $\mathrm{Kr}$ & $\mathrm{Ne}^{*}$ & $\mathrm{He}^{*}$ & $\mathrm{Ar}^{*}$ & $\mathrm{Kr}^{*}$ \\
\hline$C_{3}$ & 0.136 & 0.274 & 0.412 & 0.936 & 1.346 & 3.624 & 3.841 & 5.146 & 5.551
\end{tabular}

Table 3.2: Dispersion interaction strength $C_{3}$ in units of $\mathrm{meV} \mathrm{nm}^{3}$ for ground-state and metastable rare-gas atoms with silicon nitride. The calculations are based on the non-retarded Lifshitz formula Eq. (3.28), into which the one-oscillator approximation of the atomic polarizability Eq. (3.33), and the response of the solid, as found with the help of the Tauc-Lorentz parameterization Eq. (3.40), have been inserted. 


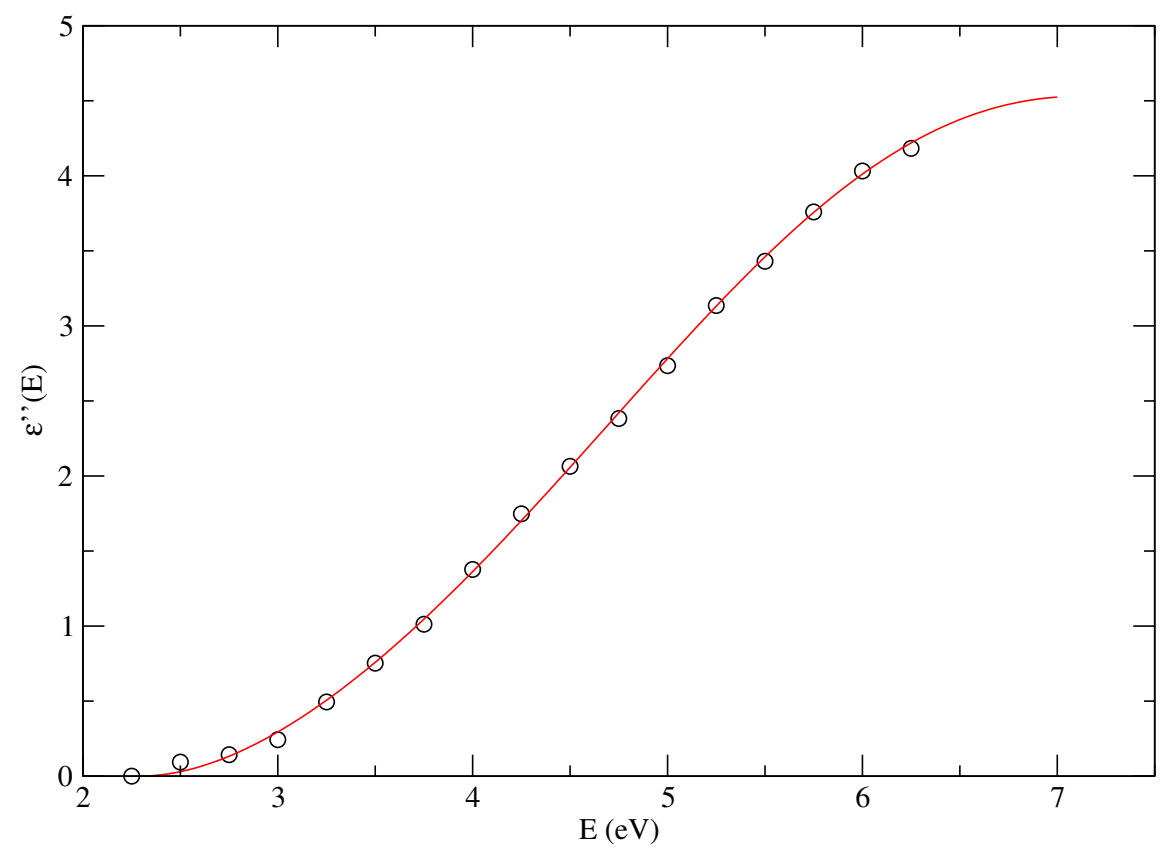

Figure 3.3: Imaginary part of the energy-dependent dielectric function of the silicon nitride grating material. The Tauc-Lorentz parameterization [97] Eq. (3.40) (solid line) with $\hbar \Omega_{\mathrm{T}}=2.29 \mathrm{eV}, \hbar A=74.5 \mathrm{eV}, \hbar \Omega=7.17 \mathrm{eV}$, and $\hbar \Gamma=7.62 \mathrm{eV}$ reproduces well the data extracted from a measurement by Savas [8.5].

\subsection{Discussion}

In Fig. 3.4 the values of $C_{3}$ for the atoms listed in Table 3.2 are plotted versus their static polarizability. One notes that for the ground-state particles the data points nearly fall on a straight line while those for metastable atoms do not. The reason for this is illustrated in Fig. 3.5 where the reduced dynamic polarizability $\tilde{\alpha}(\mathrm{i} \omega):=\frac{\alpha(\mathrm{i} \omega)}{\alpha_{0}}$ for ground-state helium, krypton and metastable helium atoms, and the reduced response $\tilde{g}(\mathrm{i} \omega):=\frac{g(\mathrm{i} \omega)}{g(0)}$ of silicon nitride are plotted versus the energy $E \equiv \hbar \omega$. In terms of these normalized quantities the dispersion interaction strength $C_{3}$ becomes

$$
C_{3}=\alpha_{0} g(0) \frac{1}{4 \pi} \int_{0}^{\infty} \mathrm{d} E \tilde{\alpha}(\mathrm{i} E) \tilde{g}(\mathrm{i} E) .
$$

This formula shows that the dispersion interaction strength $C_{3}$ of various atoms with silicon nitride depends linearly on the static atomic polarizability $\alpha_{0}$ if

$$
g(0) \frac{1}{4 \pi} \int_{0}^{\infty} \mathrm{d} E \tilde{\alpha}(\mathrm{i} E) \tilde{g}(\mathrm{i} E)=: g(0) K
$$




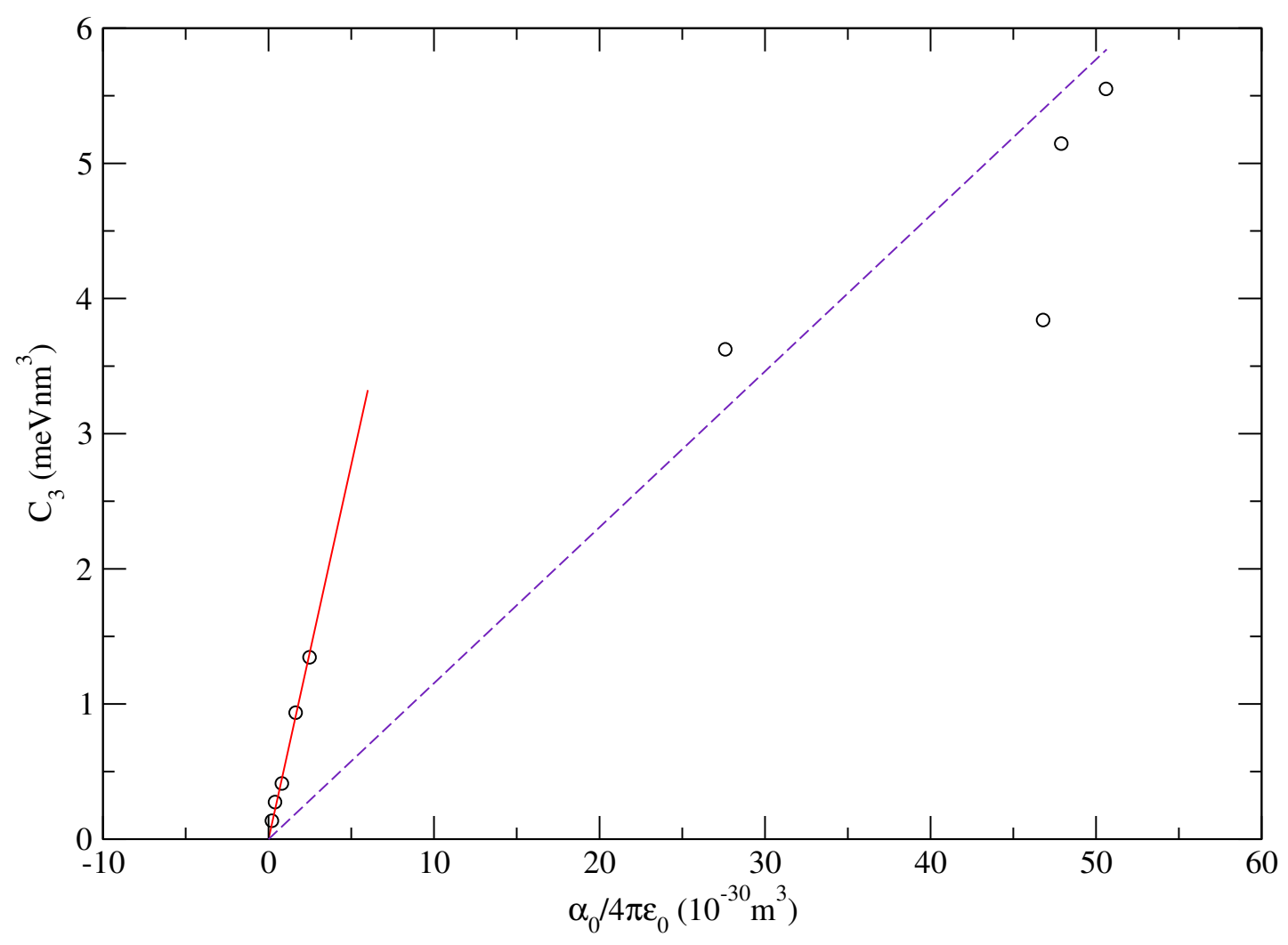

Figure 3.4: Calculated dispersion interaction strength $C_{3}$ for the atoms of Table 3.2 versus their static polarizability from Table 3.1. The data points from left to right correspond to $\mathrm{He}, \mathrm{Ne}, \mathrm{D}_{2}, \mathrm{Ar}, \mathrm{Kr}, \mathrm{Ne}^{*}, \mathrm{He}^{*}, \mathrm{Ar}^{*}, \mathrm{Kr}^{*}$. The ground-state atoms exhibit a nearly linear behaviour (solid line). A vague trend towards linearity (dashed line) can be inferred for the metastable atoms which is clearly broken by $\mathrm{He}^{*}$ (see text).

is a constant. As the solid does not change it is clear that the response $g(0)$ and $\tilde{g}(\mathrm{i} E)$ of the solid in Eq. (3.42) is the same for all atoms. The crucial point is thus if the value of the integral in Eq. (3.42) depends on the atomic species that is represented by its reduced polarizability $\tilde{\alpha}(\mathrm{i} E)$ in the integrand. It can be seen in Fig. 3.5 that for ground-state atoms that possess a high characteristic excitation energy $E_{\mathrm{a}}$ (cf. Eq. (3.33) ) the graphs of $\tilde{\alpha}(\mathrm{i} E)$ are wider than that of $\tilde{g}(\mathrm{i} E)$ whose width can also be interpreted in terms of a characteristic excitation energy within the solid. If the polarizabilities $\tilde{\alpha}(\mathrm{i} E)$ are each multiplied with the response of the solid $\tilde{g}(\mathrm{i} E)$ and integrated over all energies the value of the integral $K$ depends only weakly on the width of $\tilde{\alpha}$ so that for ground-state atoms the dependence of the dispersion interaction strength $C_{3}$ on the static atomic polarizability $\alpha_{0}$ becomes roughly linear.

If the characteristic atomic excitation energy $E_{\mathrm{a}}$ is much smaller than the width of $\tilde{g}(\mathrm{i} \omega)$ as is the case for metastable atoms the integral $K$ will crucially 
(a)

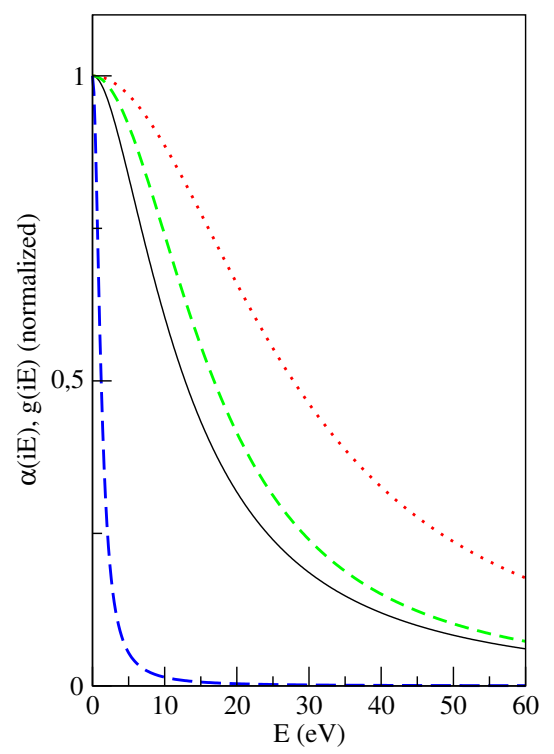

(b)

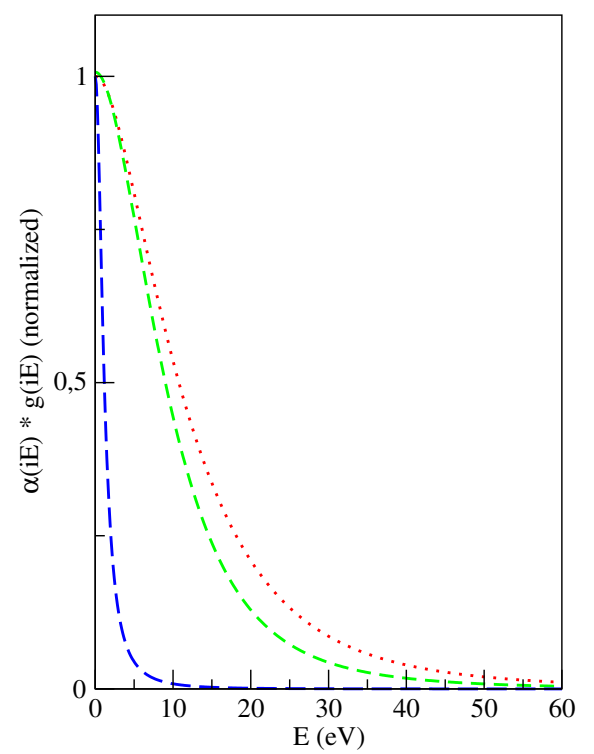

Figure 3.5: Left panel (a): Normalized atomic polarizabilities $\tilde{\alpha}(\mathrm{i} E)$ for He (dotted line), $\mathrm{Kr}$ (dashed line), He* (long dashed), and normalized response $\tilde{g}(\mathrm{i} E)$ of the solid (solid line). If the atomic curves are each multiplied by the solid's the results, which are displayed on the right panel (b), are similar for the ground-state atoms, but quite different for the metastable atom. Upon integration over all energies, the curves on the right panel for $\mathrm{He}$ and $\mathrm{Kr}$ yield approximately the same value whereas that of $\mathrm{He}^{*}$ differs considerably.

depend on $E_{\mathrm{a}}$ and the shape of $\tilde{g}(\mathrm{i} E)$ at low energies. The obvious deviation from the straight line of the data points associated with the metastable atoms in Fig. 3.4 illustrates for metastable atoms the breakdown of the previous linear approximation .

However, as Fig. 3.4 shows, the data points for the metastable species $\mathrm{Ne}^{*}$, $\mathrm{Ar}^{*}$, and $\mathrm{Kr}^{*}$ also exhibit a roughly linear behaviour whereas that of $\mathrm{He}^{*}$ breaks the trend. The reason for this becomes clear after comparing the characteristic atomic excitation energies $E_{\mathrm{a}}$ of the metastable atoms in Table 3.1. The former three metastable species are each in a ${ }^{3} \mathrm{P}_{2}$ state which brings about similar characteristic excitation energies of $E_{\mathrm{a}} \approx 2 \mathrm{eV}$. On the other hand, the metastable ${ }^{3} \mathrm{~S}_{1}$ state of $\mathrm{He}^{*}$ has a smaller characteristic energy of $E_{\mathrm{a}} \approx 1 \mathrm{eV}$. The integral $K$ is very sensitive to this difference which is why He* deviates from the roughly linear behaviour of the other metastable atoms.

In his 1980 paper Hoinkes [37] has investigated the dispersion interaction of various species of ground-state atoms with several dielectric surfaces. Empirically, 
he finds that for ground-state atoms one may approximate

$$
C_{3} \approx K \alpha_{0} g(0)
$$

where he takes $K$ (cf. Eq. (3.42)) as a constant somewhere in between $1.08 \mathrm{eV}$ and $1.41 \mathrm{eV}$. The two values are based on theoretical and experimental data, respectively. The discrepancy generally reflects the difficulties in measuring $C_{3}$ and maybe those in obtaining and extrapolating optical data for the solids. The calculations of this chapter for ground-state atoms as presented in Table 3.2 are fitted in Fig. 3.4 according to Hoinkes approximation Eq. (3.43) by a straight line of slope $K g(0)=0.554 \mathrm{eV}$. From Savas' measurement of $n$ it can be extrapolated that

$$
n(0) \approx 2 \stackrel{\text { Eq.3.48,Eq.3.49 }}{\Rightarrow} \epsilon^{\prime}(0) \approx 4 \stackrel{\text { Eq.3.47 }}{\Rightarrow} g(0) \approx 0.6,
$$

from which follows $K \approx 0.92 \mathrm{eV}$. This is in reasonable agreement with Hoinkes' result based on theoretical data $K=1.08 \mathrm{eV}$.

A systematically better approximation has been studied by Cole and Vidali. It consists in using a one-oscillator description for the response $g(\mathrm{i} E)$ of the solid, similar to that Eq. (3.33) for the atomic polarizability which the authors also employ [92]. They set

$$
g(\mathrm{i} E)=\frac{g_{0}}{1+\frac{E^{2}}{E_{\mathrm{S}}^{2}}},
$$

where $E_{\mathrm{S}}$ is a fit parameter that represents a characteristic transition energy within the solid, and $g_{0}$ is another parameter that can be set equal to the static limit of $g(\mathrm{i} E)$ but is used by the authors as a free parameter which in practice helps to compensate for the missing account of the optical band gaps of the solids.

Fig. 3.6 compares $g(\mathrm{i} E)$ as obtained from the Tauc-Lorentz formula for silicon nitride with the one-oscillator approximation Eq. (3.45) while $g_{0}$ is identified with the static limit $g(0)=0.588$ of the result of the Tauc-Lorentz parameterization. The one-oscillator approximation is best fitted to the more exact curve with a characteristic excitation energy $E_{\mathrm{S}}=13.47 \mathrm{eV}$ for the solid.

On using one-oscillator approximations for both the atomic polarizability $\alpha(\mathrm{i} E)$ Eq. (3.33) and the response $g(\mathrm{i} E)$ of the solid Eq. (3.45) the integral Eq. (3.41) that represents the dispersion coefficient $C_{3}$ becomes analytically accessible. One has

$$
C_{3}^{\mathrm{VC}}=\frac{1}{4 \pi} \alpha_{0} g(0) \int_{0}^{\infty} \mathrm{d} E \frac{1}{\frac{E^{2}}{E_{\mathrm{S}}^{2}}} \frac{1}{1+\frac{E^{2}}{E_{\mathrm{a}}^{2}}}=\frac{\alpha_{0} g(0) E_{\mathrm{a}} E_{\mathrm{S}}}{8\left(E_{\mathrm{a}}+E_{\mathrm{S}}\right)} .
$$

In view of the failure of the linear approximation for metastable atoms as described above it is interesting to see if Eq. (3.46) can be applied to both groundstate and metastable atoms. If $g_{0}$ is kept fixed to the static limit $g(0)=0.588$ 


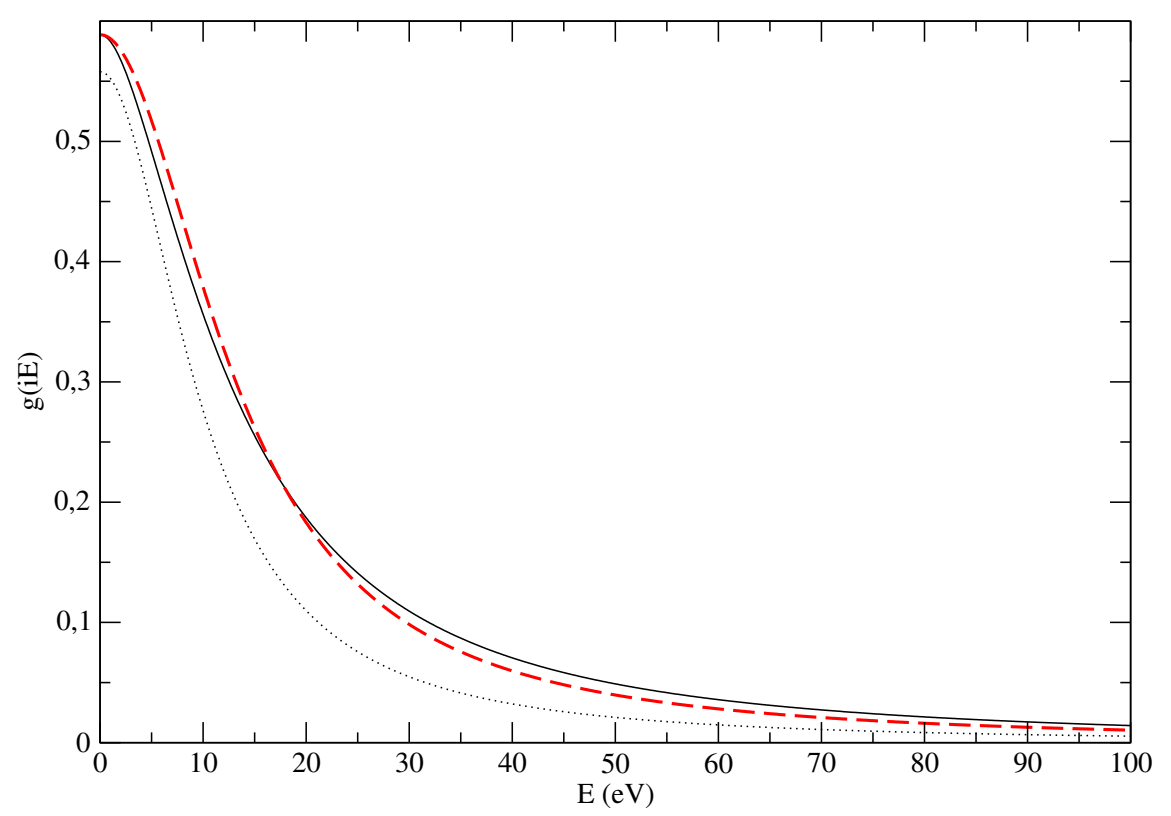

Figure 3.6: The response $g(\mathrm{i} E)$ of the solid as extrapolated from optical data (solid line) is well fitted by the one-oscillator approximation Eq. (3.45) with $g_{0}=g(0)=0.588$ fixed and $E_{\mathrm{S}}=13.43 \mathrm{eV}$ (dashed line). From experimental values of $C_{3}$ [12] the dotted curve has been derived, which clearly underestimates the response as given by the other curves.

of the result of the Tauc-Lorentz parameterization, and $E_{\mathrm{S}}$ is determined by a least-square fit of Eq. (3.46) to the values of $C_{3}$ from Table 3.2 one obtains $E_{\mathrm{S}}=14.31 \mathrm{eV}$, which is in reasonable agreement with the value $E_{\mathrm{S}}=13.47 \mathrm{eV}$ that has been extracted from a direct fit of the one-oscillator approximation Eq. (3.45) of $g(\mathrm{i} E)$ to the Tauc-Lorentz result (cf. Fig. 3.6). The absolute value of the relative error $\gamma:=\frac{C_{3}^{\mathrm{VC}}-C_{3}}{C_{3}}$ is less than $7 \%$ throughout (see Table 3.3), so it turns out that Vidali and Cole's formula Eq. (3.46) is suitable to describe both ground-state and metastable atoms.

It is remarkable that the $C_{3}^{\mathrm{VC}}$ values for the metastable atoms are smaller than those obtained with the Tauc-Lorentz parameterization. From Fig. 3.6 one could expect that for metastable atoms whose polarizability at imaginary energies has a narrow peak at low energies, $C_{3}$ according to Eq. (3.41) would be comparatively larger as the one-oscillator approximation of $g(\mathrm{i} E)$ is larger than the exact curve for low energies. However, the high energy contributions where the approximation runs below the exact curve more than compensate for that.

For the sake of completeness it shall be mentioned that it is possible to keep $g_{0}$ fixed as before, but extract $E_{\mathrm{S}}$ from a fit to the ground-state atoms only. Another method is to directly follow Cole and Vidali's example and apply a fit 


\begin{tabular}{c||c|c|c|c|c|c|c|c|c} 
atom & $\mathrm{He}$ & $\mathrm{Ne}$ & $\mathrm{D}_{2}$ & $\mathrm{Ar}$ & $\mathrm{Kr}$ & $\mathrm{Ne}^{*}$ & $\mathrm{He}^{*}$ & $\mathrm{Ar}^{*}$ & $\mathrm{Kr}^{*}$ \\
\hline \hline$C_{3}$ & 0.136 & 0.274 & 0.412 & 0.936 & 1.346 & 3.624 & 3.841 & 5.146 & 5.551 \\
\hline$C_{3}^{\mathrm{VC}}$ & 0.143 & 0.283 & 0.432 & 0.942 & 1.429 & 3.603 & 3.789 & 5.101 & 5.505 \\
$\gamma(\%)$ & 5.1 & 3.3 & 4.9 & 0.6 & 6.2 & -0.6 & -1.4 & -0.9 & -0.8 \\
\hline$C_{3}^{\mathrm{VC}}$ & 0.136 & 0.269 & 0.415 & 0.901 & 1.370 & 3.568 & 3.767 & 5.061 & 5.546 \\
$\gamma(\%)$ & 0 & 1.8 & 0.7 & -3.7 & -1.8 & -1.5 & -1.9 & -1.6 & -0.1 \\
\hline$C_{3}^{\mathrm{VC}}$ & 0.135 & 0.267 & 0.416 & 0.901 & 1.371 & 3.625 & 3.834 & 5.147 & 5.553 \\
$\gamma(\%)$ & -0.7 & -2.6 & 1.0 & -3.7 & 1.9 & $<0.1$ & -0.2 & $<0.1$ & $<0.1$
\end{tabular}

Table 3.3: Dispersion interaction strength $C_{3}$ in units of $\mathrm{meV} \mathrm{nm}^{3}$. Top row: Theoretical values based on the Tauc-Lorentz parameterization. First double row: Eq. (3.46) fitted to the theoretical values with $g_{0}=0.588$ fixed, $E_{\mathrm{S}}=14.31 \mathrm{eV}$, and relative error. Second double row: Analogous. Fit to ground-state atoms only; $g_{0}=0.588$ fixed, $E_{\mathrm{S}}=13.26 \mathrm{eV}$. Third double row: Analogous. Fit to all atoms with two fit parameters $g_{0}=0.601$ and $E_{\mathrm{S}}=12.78 \mathrm{eV}$

to all atoms with both $g_{0}$ and $E_{\mathrm{S}}$ as fit parameters. Both methods yield results of a similarly good quality to the method first discussed. It is worth noting that the best fit is obtained with two parameters where $g_{0}$ comes out as 0.601 , which could have been estimated directly from Savas' optical data, as shown in Fig. 3.2 and demonstrated in Eq. (3.44). The details of both alternative fitting procedures can be also be read from Table 3.3 .

In sum, if both Hoinkes' and Vidali and Cole's approximations are evaluated in terms of the optical data as provided by Savas they lead to values of the dispersion interaction strength $C_{3}$ that are in good agreement with those calculated with the help of the Tauc-Lorentz parameterization.

The values of the dispersion interaction strength $C_{3}$ for the ground-state particles $\mathrm{He}, \mathrm{Ne}, \mathrm{D}_{2}, \mathrm{Ar}$, and $\mathrm{Kr}$ that have been measured by Grisenti et al. [12] have not yet been compared to theoretical values in quantitative terms. With the help of the theoretical values as in Table 3.2 such a comparison is now possible and is displayed in Table 3.4. It turns out that the experimental values are all about $20 \%$ smaller than the theoretical ones. In the following the experimental results are analyzed in terms of Hoinkes' and Vidali and Cole's approximations. The parameters that fit the approximations to the experiment are then interpreted in terms of the optical properties of the solid and compared to the optical data that have been provided by the manufacturers of the transmission grating. It will then be possible to draw a conclusion as to whether the experimental values are systematically too small or the theoretical ones are too large.

In their paper [12] the authors have demonstrated that the measured values of 


\begin{tabular}{c||c|c|c|c|c} 
atom & $\mathrm{He}$ & $\mathrm{Ne}$ & $\mathrm{D}_{2}$ & $\mathrm{Ar}$ & $\mathrm{Kr}$ \\
\hline$C_{3}$ theo. & 0.136 & 0.274 & 0.412 & 0.936 & 1.346 \\
$C_{3}$ exp. & $0.10 \pm 0.02$ & $0.21 \pm 0.04$ & $0.34 \pm 0.09$ & $0.71 \pm 0.12$ & $1.09 \pm 0.17$ \\
$\gamma(\%)$ & -26.5 & -23.4 & -17.5 & -24.1 & -19.0
\end{tabular}

Table 3.4: Dispersion interaction strength $C_{3}$ in units of $\mathrm{meV} \mathrm{nm}^{3}$. First row: Theoretical value based on the Tauc-Lorentz parameterization. Second row: Measured values [12]. The third row shows that the experimental values are all about $20 \%$ smaller than predicted by theory.

the dispersion interaction strength $C_{3}$ of $\mathrm{He}, \mathrm{Ne}, \mathrm{D}_{2}, \mathrm{Ar}$, and $\mathrm{Kr}$ with amorphous silicon nitride exhibit a linear behaviour when plotted versus the static atomic polarizability $\alpha_{0}$, which is in agreement with Hoinkes' rule Eq. (3.43). The straight line through the origin that has been fitted to the data by the authors has a slope $g(0) K=0.440 \mathrm{eV}$. Adopting Hoinkes' lower limit for $K=1.08 \mathrm{eV}$ one obtains a maximal $g(0)=0.407$. The static limit of the index of refraction $n(E)$ can be derived from this result by re-arranging Eq. (3.26) which yields

$$
\lim _{E \rightarrow 0} \epsilon(\mathrm{i} E)=\frac{1+g(0)}{1-g(0)} .
$$

From the Kramers-Kronig relation Eq. (3.30) it is evident that

$$
\lim _{E \rightarrow 0} \epsilon(\mathrm{i} E)=\lim _{E \rightarrow 0} \epsilon^{\prime}(E) .
$$

According to Eq. (3.38) the static index of refraction $n(0)$ is given by

$$
n(0)=\sqrt{\epsilon^{\prime}(0)}
$$

where the contribution from the static limit of the absorption coefficient $\kappa(0)$ has been ignored because due to the optical band gap its value is zero. This leads to $n(0)=1.58$ which is clearly below the static limit of $n(0) \approx 2$ that the optical measurement Fig. 3.2 suggests.

Alternatively, one might interpret the slope of the experimental curve with the help of the optical data which yields $g(0) \approx 0.6$. Then one obtains as a result $K=0.73$ which is about $25 \%$ below the lowest of Hoinkes' values, namely $K=1.08$.

The second approximate method to interpret the measured values of $C_{3}$ is Vidali and Cole's who use the formula Eq. (3.46). If this equation is fitted to the experimental data one obtains for the two fit parameters $g_{0}=0.558$ and $E_{\mathrm{S}}=9.9 \mathrm{eV}$. The one-oscillator approximation Eq. (3.45) for the response $g(\mathrm{i} E)$ of the solid is plotted for these two values in Fig. 3.6. It is evident that the 
response curve derived from the experimental values of $C_{3}$ underestimates the response that follows from the extrapolation of the optical data.

It is concluded that the measured values of $C_{3}$ are lower than can be expected from the optical data of the amorphous silicon nitride material. In section 5.1.3 the process that determines $C_{3}$ in Ref. [12] is briefly reviewed. Thereby a plausible explanation of the deviations becomes apparent and is presented to the reader.

\subsection{Summary}

In order to provide for the first time accurate theoretical values of the dispersion interaction strength $C_{3}$ of atoms with the transmission gratings' silicon nitride material the following issues have been clarified in the course of this chapter.

- The dispersion interaction in the retarded and in the non-retarded regime between atoms can be derived from a general formula that is rooted in quantum electrodynamics.

- On treating the dielectric grating material as a continuum and neglecting the scattering of virtual photons at the interface vacuum-dielectric the quantum electrodynamical formalism can be be applied to atoms interacting with an idealized dielectric surface, for both the retarded and the non-retarded regime.

- The interaction constant $C_{3}$ is calculated in the dipole approximation as a function of the frequency-dependent atomic polarizability and the electronic response of the solid. In the retarded case, $C_{3}$ depends on the distance between the atom and the surface, whereas in the non-retarded case the potential follows a power law with the inverse cube of the atom-surface distance.

- The frequency-dependent atomic polarizability is determined semi-empirically within a suitable one-oscillator model.

- The response of the dielectric solid is determined semi-empirically with the Tauc-Lorentz parameterization which is a one-ocillator model that also takes explicit account of the optical band gap. As experimental input the energy-dependent index of refraction $n(E)$ and extinction coefficient $\kappa(E)$ in the region between $1 \mathrm{eV}$ and $6 \mathrm{eV}$ are sufficient.

- Within certain limits the accurate calculation of the dispersion interaction strength $C_{3}$ of atoms with silicon nitride, as a function of the static atomic polarizability, is in good agreement with a simple linear approximation and with an approximation that ignores the band gap of the solid. 
- The calculated results, together with available optical data on the grating material suggest that previously published measured values of $C_{3}$ are subject to a systematical error of $30 \%$. The origin of the error is in chapter 5 traced back to a misinterpretation of data concerning the grating bar geometry. 


\section{Chapter 4}

\section{Inclusion of Atom-Surface Potentials}

After the origin of the attractive dispersion interaction has been clarified in the last chapter, the following sections demonstrate how to implement the dispersion interaction for a particular transmission grating in order to evaluate the scattering theoretical result Eq. (2.59) for the detection probability. The shape of the grating bars is taken account of by a new integration method which can be generalized easily to describe diffraction with inclined incidence, adsorbate layers on the bar surface, or interaction potentials with different power laws as regards the atom-surface distance. Furthermore, it is investigated for the first time how the retarded atom-surface potential Eq. (3.25) can be specified for the grating bar geometry and if the retarded potential can be inferred from measured diffraction intensities.

\subsection{Phase Shift}

The scattering theoretical result Eq. (3.25) for the detection probability $I_{\Omega}(\mathbf{r})$ requires the knowledge of the quantum mechanical amplitude $\psi(x, 0)$ at the rear side of the transmission grating inside a slit of width $s$ which is centred around the origin of the chosen coordinate frame (cf. Fig.4.1). According to its definition Eq. (2.61) the wavefunction $\psi(x, 0)$ is a complex exponential whose phase reads

$$
\varphi(x):=-\frac{1}{\hbar v} \int_{-\infty}^{+\infty} \mathrm{d} z V^{\mathrm{att}}(x, z) .
$$

The potential $V^{\text {att }}(x, z)$ between an atom and the two bars on each side of the slit is evaluated in Eq. (4.1) along a straight trajectory parallel to the $z$ axis, which 


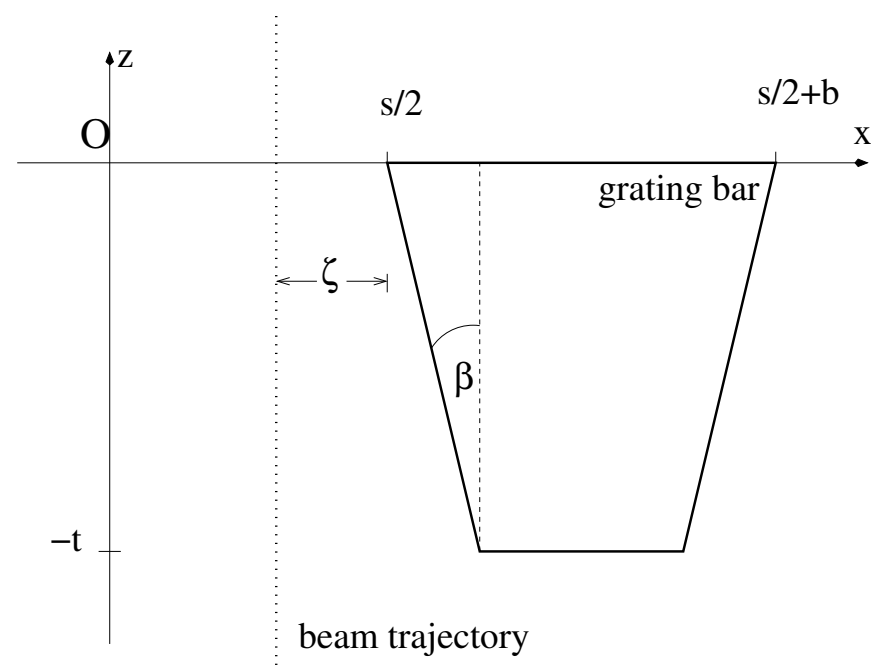

Figure 4.1: Theoretical sketch of an atomic beam scattered off a single trapezoidshaped grating bar. The straight line labeled "beam trajectory" supports the visualization of the newly defined impact parameter $\zeta$. It represents one of the possible paths an atom can follow on its way through the grating. In reality, $b \approx 30 \mathrm{~nm}$ while the de Broglie wavelength $\lambda$ of the incident particles is about 300 times smaller.

also marks the direction of the incident beam. When regarding this situation illustrated in Fig. 4.1 it should be borne in mind that the mentioned trajectory is just one out of many possible paths the atom could go through the grating. The interaction of the atom with the grating bars is evaluated quantum mechanically in terms of all possible paths. The only physical reality about the trajectories is their all being possible in the present experiments. The trapezoid-shaped idealization of the bar cross-section as shown in Fig. 4.1 is chosen according to a scanning electron micrograph Fig. 4.2 taken by T. Savas of one of his transmission gratings.

Regarding the atom-surface potential $V^{\text {att }}(x, z)$ that appears in Eq. (4.1) it is possible to go beyond the approximate concept introduced in Fig. 2.23 for an atom at a distance $l$ from a dielectric semi-infinite half-space. There the attractive part of the potential is taken to arise solely from the non-retarded dispersion interaction

$$
V_{3}(l)=\frac{-C_{3}}{l^{3}},
$$

while the repulsion is modelled as a rectangular barrier placed at the distance $l_{0}$ from the surface where the attraction and the repulsion cancel. In view of the formula Eq. (3.25) it is now possible to also include the repulsive part

$$
V_{9}(l)=\frac{C_{9}}{l^{9}}
$$




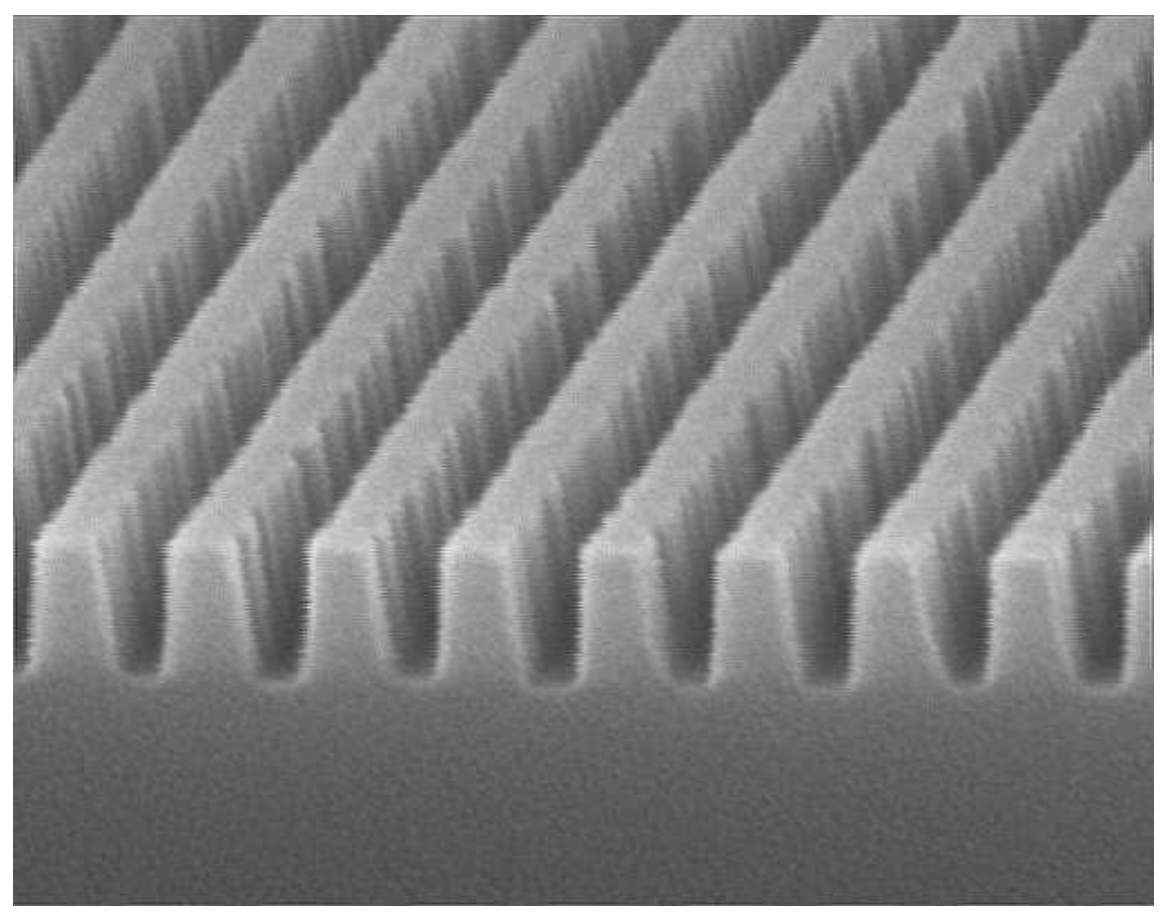

Figure 4.2: Scanning electron micrograph of the grating bars before they are removed from the substrate during the manufacturing process, taken by Tim Savas, MIT. The regular structure of a period of $100 \mathrm{~nm}$ and the trapezoid-like cross-section of the bars is clearly visible.

so that the correct shape of the potential well near the surface can be taken account of by adding to the integrand in Eq. (4.1) the appropriate potential $V^{\text {rep }}(x, z)$. This may be done because for small $l \leq l_{0}$ the repulsion increases very quickly with $-l$ which leads to a sharp rise of the phase Eq. (4.1). The wavefunction $\psi(x, 0)$ in the slit is the complex exponential of that sharply rising phase so it oscillates rapidly for atom-surface distances that fall in the realm of the repulsion. Upon integration over the slit as in Eq. (3.25) the regions of rapid oscillations contribute zero to the detection probability hence the situation is nearly equivalent to the slit being narrowed by twice $l_{0}$. What makes a difference, though, is that the dispersion interaction is calculated with respect to $l$ and not $l-l_{0}$ which would be the case if the slit was really narrowed by $2 l_{0}$. This qualitative argument remains valid if the semi-infinite half-space is replaced by the finite grating bar cross-section as is shown in the following. The fundamental advantage of the inclusion of the repulsive potential is that for the first time the atom-bar potential can be modelled realistically which opens the way to measuring the real atom-surface potential by means of atom diffraction.

The $V_{3}$ potential given in Eq. (4.2) refers to the standard situation of an infinite half-space $\left\{\mathbf{r}^{\prime} \in \mathbb{R} \mid x^{\prime}<0\right\}$ each volume element of which interacts with 
an atom outside at $\mathbf{r}$ via an attractive potential of the form

$$
W_{6}(\mathbf{r}):=\frac{-K_{6}}{\left|\mathbf{r}-\mathbf{r}^{\prime}\right|^{6}}
$$

with $K_{6}>0$. Without restricting generality the atom can be placed at $\mathbf{r}=$ $(l, 0,0)^{\mathrm{T}}$. Then one finds by standard integration methods

$$
V_{3}(l)=\int_{-\infty}^{+\infty} \mathrm{d} y^{\prime} \int_{-\infty}^{+\infty} \mathrm{d} z^{\prime} \int_{-\infty}^{0} \mathrm{~d} x^{\prime} \frac{-K_{6}}{\left[\left(l-x^{\prime}\right)^{2}+\left(y^{\prime}\right)^{2}+\left(z^{\prime}\right)^{2}\right]^{3}}=\frac{-K_{6} \pi}{6 l^{3}}=: \frac{-C_{3}}{l^{3}} .
$$

The repulsive $V_{9}$ potential in Eq. (4.3) can similarly be expressed as arising from an interaction between the atom and the volume elements of the solid, which has the form

$$
W_{12}(\mathbf{r}):=\frac{K_{12}}{\left|\mathbf{r}-\mathbf{r}^{\prime}\right|^{12}} .
$$

One obtains as an analogon to Eq. (4.5)

$$
V_{9}(l)=\frac{K_{12} \pi}{45 l^{9}}=: \frac{C_{9}}{l^{3}}
$$

as is demonstrated in the appendix for both the attractive and the repulsive case.

In order to incorporate the geometry of one bar within the concept just set out the limits of the integrals involved in Eq. (4.5) and Eq. (4.7) are modified such as to enclose the volume of the trapezoidal prism that forms a grating bar. Hereby the the boundaries in $y$-direction are known to be far away from the scattering plane and can be conveniently set to $\pm \infty$, respectively. The other boundaries can be deduced elementarily from Fig.4.1. It turns out that the phase shift $\varphi(x)$ defined in Eq. (4.1) is the sum

$$
\begin{aligned}
\varphi(x)=: & \varphi_{\mathrm{r}}(x)+\varphi_{\mathrm{l}}(x) \\
=: & -\frac{1}{\hbar v} \int_{-\infty}^{+\infty} \mathrm{d} z\left[V_{\mathrm{r}}^{\mathrm{att}}(x, z)+V_{\mathrm{r}}^{\mathrm{rep}}(x, z)+V_{\mathrm{l}}^{\mathrm{att}}(x, z)+V_{1}^{\mathrm{rep}}(x, z)\right]
\end{aligned}
$$

of the the respective contributions from the bars on the right and on the left side of the slit, each of which consists of an attractive and a repulsive part. The potentials are given by the equations

$$
V_{\mathrm{r}}^{\mathrm{att}}(x, z)=-\frac{6 C_{3}}{\pi} \int_{-t}^{0} \mathrm{~d} z^{\prime} \int_{-\infty}^{+\infty} \mathrm{d} y^{\prime} \int_{\frac{s}{2}-z^{\prime} \tan \beta}^{\frac{s}{2}+b+z^{\prime} \tan \beta} \mathrm{d} x^{\prime}\left[\left(x-x^{\prime}\right)^{2}+\left(y^{\prime}\right)^{2}+\left(z-z^{\prime}\right)^{2}\right]^{-3}
$$




$$
\begin{aligned}
& V_{\mathrm{r}}^{\mathrm{rep}}(x, z)=\frac{45 C_{9}}{\pi} \int_{-t}^{0} \mathrm{~d} z^{\prime} \int_{-\infty}^{+\infty} \mathrm{d} y^{\prime} \int_{\frac{s}{2}-z^{\prime} \tan \beta}^{\frac{s}{2}+b+z^{\prime} \tan \beta} \mathrm{d} x^{\prime}\left[\left(x-x^{\prime}\right)^{2}+\left(y^{\prime}\right)^{2}+\left(z-z^{\prime}\right)^{2}\right]^{-6} \\
& V_{1}^{\mathrm{att}}(x, z)=-\frac{6 C_{3}}{\pi} \int_{-t}^{0} \mathrm{~d} z^{\prime} \int_{-\infty}^{+\infty} \mathrm{d} y^{\prime} \int_{\frac{s}{2}-z^{\prime} \tan \beta}^{\frac{s}{2}+b+z^{\prime} \tan \beta} \mathrm{d} x^{\prime}\left[\left(x-x^{\prime}\right)^{2}+\left(y^{\prime}\right)^{2}+\left(z-z^{\prime}\right)^{2}\right]^{-3}
\end{aligned}
$$

and

$$
V_{1}^{\mathrm{rep}}(x, z)=\frac{45 C_{9}}{\pi} \int_{-t}^{0} \mathrm{~d} z^{\prime} \int_{-\infty}^{+\infty} \mathrm{d} y^{\prime} \int_{\frac{s}{2}-z^{\prime} \tan \beta}^{\frac{s}{2}+b+z^{\prime} \tan \beta} \mathrm{d} x^{\prime}\left[\left(x-x^{\prime}\right)^{2}+\left(y^{\prime}\right)^{2}+\left(z-z^{\prime}\right)^{2}\right]^{-6}
$$

On inserting these four expressions into Eq. (4.8) and subsequent evaluation of the integrals as demonstrated in the appendix one obtains for the phase $\varphi_{\mathrm{r}}(x)$ arising from the potential of the right bar

$$
\begin{aligned}
\varphi_{\mathrm{r}}(x) & =\frac{C_{3}}{2 \hbar v \tan \beta}\left\{\frac{1}{\zeta^{2}}-\frac{1}{(\zeta+t \tan \beta)^{2}}+\frac{1}{(\zeta+b)^{2}}-\frac{1}{(\zeta+b-t \tan \beta)^{2}}\right\}- \\
& -\frac{C_{9}}{8 \hbar v \tan \beta}\left\{\frac{1}{\zeta^{8}}-\frac{1}{(\zeta+t \tan \beta)^{8}}+\frac{1}{(\zeta+b)^{8}}-\frac{1}{(\zeta+b-t \tan \beta)^{8}}\right\},
\end{aligned}
$$

where the impact parameter $\zeta:=\frac{s}{2}-x$ with respect to the right slit boundary has been introduced (cf. Fig. 4.1). One obtains the contribution $\varphi_{1}$ from the left bar by simply replacing $\zeta$ with $s-\zeta$. The interested reader may check the progress of the calculation summarized by Eq. (4.14) in the appendix where it is shown that similar formulae can be easily derived for all potentials of the form

$$
V_{n}(l)=\frac{ \pm C_{n}}{l^{n}} \quad, \quad n \in\{2,3,4, \ldots\},
$$

or a sum of these potentials. Fig. 4.3 shows a typical example of the result Eq. (4.14) for helium atoms and a silicon nitride grating like those used in the experiment.

The first term of the sum in Eq. (4.14) can be rewritten as

$$
\frac{t C_{3}}{\hbar v \zeta^{3}}\left\{\frac{1+\frac{t \tan \beta}{2 \zeta}}{\left(1+\frac{t \tan \beta}{\zeta}\right)^{2}}-\frac{1}{\left(1+\frac{b}{\zeta}\right)} \frac{1+\frac{t \tan \beta}{2 \zeta\left(1+\frac{b}{\zeta}\right)}}{\left(1+\frac{t \tan \beta}{\zeta\left(1+\frac{b}{\zeta}\right)}\right)^{2}}\right\} .
$$




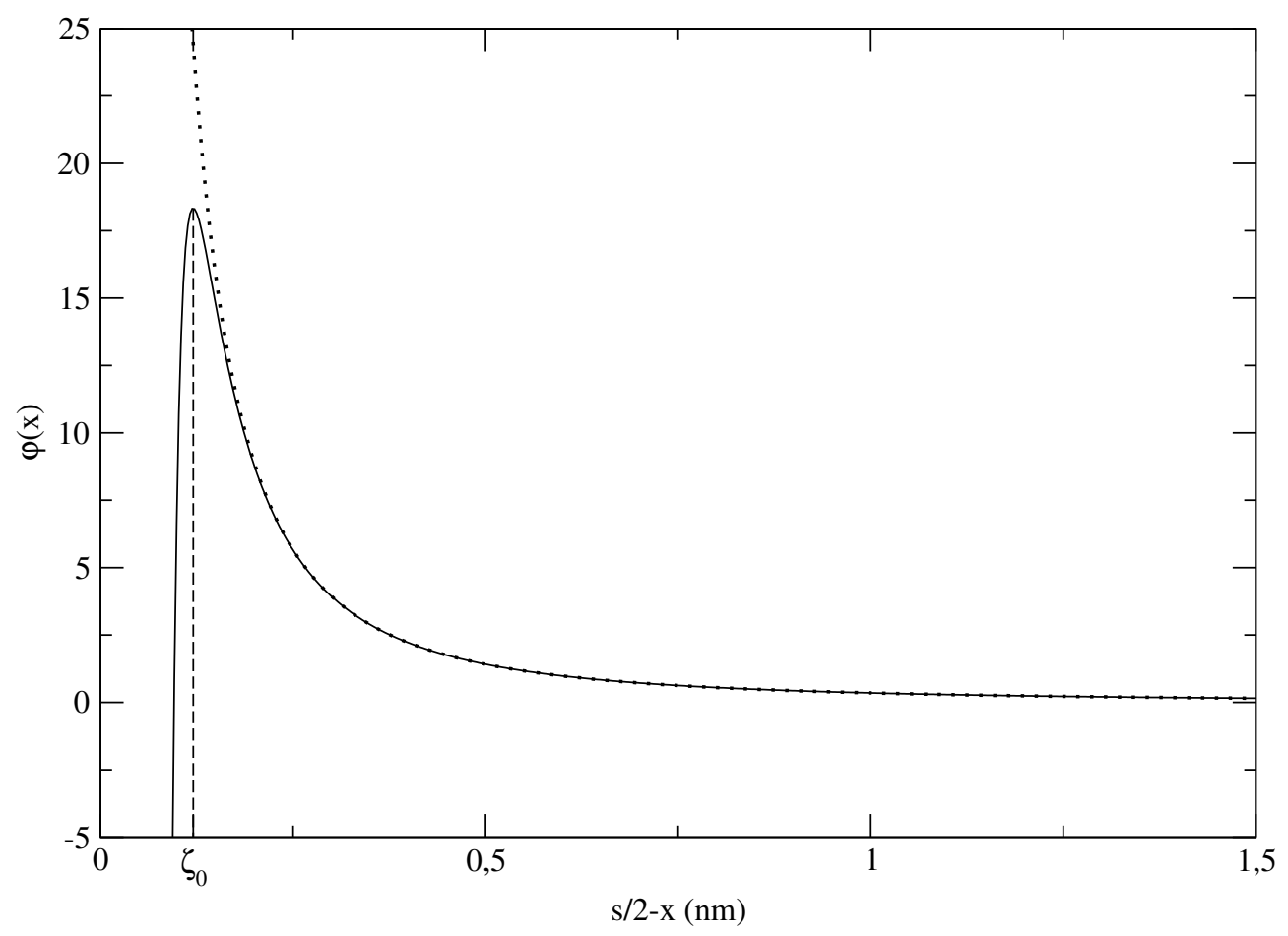

Figure 4.3: The quantum mechanical phase $\varphi_{\mathrm{r}}(x)$ (solid line) arising from the interaction between the atomic beam and the grating bar on the right of the slit is plotted as a function of the distance between the beam trajectories (quantum mechanical paths) and the protruding trailing edge at $x=\frac{s}{2}$ of the bar (cf. Fig.4.1). For large distances the contribution (dotted line) from the attractive potential dominates. At $\frac{s}{2}-x=\zeta_{0}$ (dashed line) the influence of the repulsive potential produces a local maximum of the phase.

It is possible to ascribe the first term in this result to the side of the bar facing the slit, while the second term accounts for the far side. It can be seen that for very wide bars $b \rightarrow \infty$ the latter term vanishes. Within this limit the result Eq. (4.16) is comparable to that presented in [12] which contains an additional factor $\cos \beta$. The difference is due to the approximate method of integration which has been employed in the cited reference and which uses only a part of the trajectory of length $\approx t$ whereas in Eq. (4.1) the trajectory correctly extends from minus to plus infinity. The correction that arises from this is a factor of about $2 \%$ to the phase $\varphi(x)$. In anticipation of a later result it may be said that this leads to an average error of $0.2 \%$ in the diffraction intensities which is negligibly small. Furthermore, the approximate integration method [12] proves useful when it comes to retarded bar potentials (see section 4.4) whose structure is somewhat more complicated than the potentials' treated so far.

Varying the wedge angle $\beta$ in Eq. (4.14) it is possible to account for different 
grating bar cross-section shapes ranging from triangles to rectangles, provided their base line is perpendicular to the incident beam. A completely arbitrary bar shape can be evaluated by decomposing the shape into a sum of small triangles and rectangles whose base lines have the above stated property. If the base line is no longer perpendicular to the incident beam the integration limits in Eq. (4.14) assume a complicated intertwined structure that hinders a straightforward analytical solution. However, it will be shown below that there is a simple way to modify Eq. (4.14) for the case that the grating is rotated around the vertical axis.

With the above result for the phase $\varphi(x)$ that describes the influence of the atom-surface potentials inside a grating slit the diffraction problem is practically solved! For a perfect grating whose bars are homogeneous trapezoidal prisms, and for an incident beam that is an incoherent superposition of plane waves of various velocities and angles of incidence, each of the plane waves leads to an intensity $I_{\Omega}\left(\vartheta_{n}\right)$ of the $n$th diffraction order

$$
I_{\Omega}\left(\vartheta_{n}\right) \propto\left|f_{\text {slit }}\left(\vartheta_{n}\right)\right|^{2},
$$

with the slit amplitude

$$
f_{\text {slit }}\left(\vartheta_{n}\right)=\int_{-\frac{s}{2}}^{\frac{s}{2}} \mathrm{~d} x^{\prime} \mathrm{e}^{-\mathrm{i} \kappa_{n} x^{\prime}} \mathrm{e}^{\mathrm{i} \varphi\left(x^{\prime}\right)}
$$

and where the phase $\varphi\left(x^{\prime}\right)$ is given by Eq. (4.8) and Eq. (4.14).

Besides this phase that arises from the atom-bar interaction, the integrand in Eq. (4.18) displays a geometrical phase shift $k x^{\prime} \sin \vartheta_{n} \equiv \kappa_{n} x^{\prime}$. The latter reflects the fact that for an incident plane wave which arrives at all points $x^{\prime}$ of the grating's trailing edge at the same time, the waves going out from $x^{\prime}$ at an angle $\vartheta_{n}$ are advanced or retarded by $x^{\prime} \sin \vartheta_{n}$ with respect to those emitted in the origin. Looking at typical numbers the geometrical phase shift is equal to $2 \pi$ for $x^{\prime} \approx s$ so the first exponential in Eq. (4.18) only runs through a few cycles across the slit. By contrast, the second exponential oscillates rapidly if $\varphi\left(x^{\prime}\right)$ changes fast with $x^{\prime}$ as is the case for trajectories passing close to the bar surfaces. Note that the speed of oscillation does not depend on the absolute value of $\varphi(x)$ but on its derivative with respect to $x$. As the impact parameter $\zeta$ tends to zero the phase shift $\varphi_{\mathrm{r}}(x)$ tends to $-\infty$ at increasing speed, and the associated exponential in this limit oscillates infinitely fast. As any fast oscillations formally cancel out when integrated over the divergence has no conceptual consequences for the result Eq. (4.14). This is intuitively understandable recalling that the atomic trajectories cannot even in theory be taken as infinitely narrow but rather of a finite width which in atom optics is set equal to the de Broglie wavelength $\lambda$ of the atom. If the phase $\varphi(x)$ given by Eq. (4.8) and Eq. (4.14) changes by more than a full cycle $2 \pi$ over a distance $\Delta x=\lambda$ the trajectory as part of the quantum 


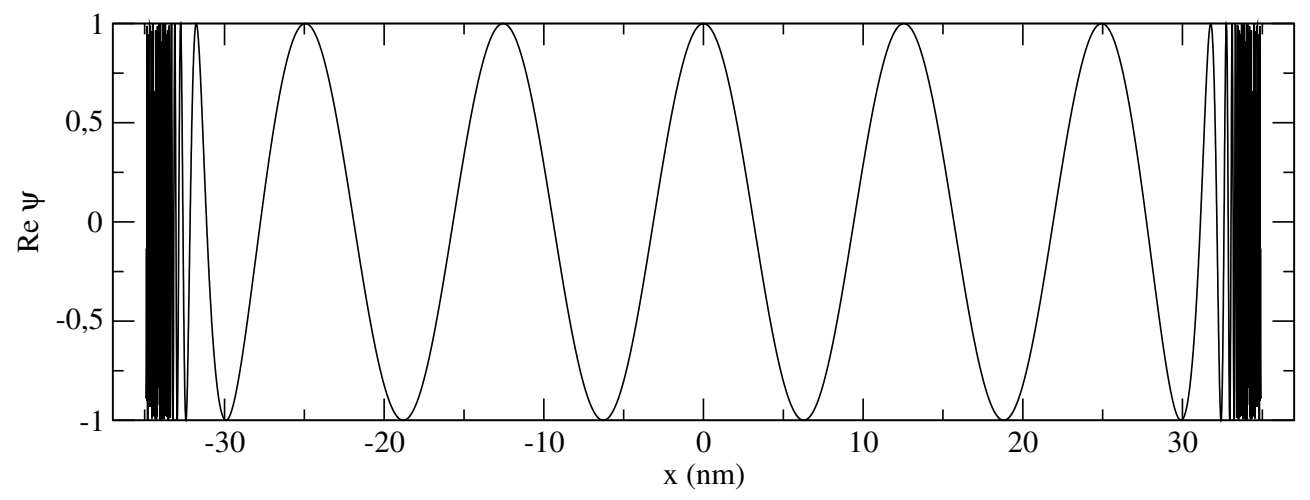

Figure 4.4: The real part of the integrand of Eq. (4.18) is here denoted by $\operatorname{Re}(\Psi)$ and plotted versus the integration variable $x$ that is a measure for the distance across the slit. Integrated over $x$, the function $\Psi$ yields the slit amplitude $f_{\text {slit }}\left(\vartheta_{1}\right)$ for the first principal diffraction order. The slow oscillations arise from the geometrical phase shift (see text), the fast oscillations near the edges of the $70 \mathrm{~nm}$ wide slit are due to the atom-bar interaction.

mechanical ensemble cannot contribute a definite phase to the amplitude at the detector and, in effect, vanishes. Fig. 4.4 shows the real part of the integrand in Eq. (4.18).

According to Eq. (4.17) the intensity $I_{\Omega}\left(\vartheta_{n}\right)$ of the $n$th principal diffraction order is equal to the modulus squared of the slit amplitude $f_{\text {slit }}\left(\vartheta_{n}\right)$. Fig. 4.5 shows a plot of $\left|f_{\text {slit }}(\vartheta)\right|^{2}$ versus the continuous diffraction angle $\vartheta$. The same plot is in Fig. 4.6 compared to an analogous case but without any atom-bar potential beyond a strongly repulsive core at the very bar surface. It can be interpreted as if arising from the diffraction of an X-ray beam that has the same wavelength as the atomic beam. One notes that the atom-bar potential leads to an effective narrowing of the slit so that the slit function becomes wider. Furthermore the sharp minima of the optical case are removed because for atoms approaching the surface the onset of the potential happens slowly as compared to the abrupt barrier the X-ray photons experience on the bar surface. These observations noted here for didactic purposes are explained in more detail in [12].

It remains to show how the results given so far for a monochromatic incident atomic beam apply to the real experimental beam which is an incoherent superposition of many monochromatic atomic states which possess different angles of incidence $\vartheta_{\text {inc }}$ and different velocities $v$. In chapter 2 (cf. Eq. (2.66)) it has been anticipated that the relative intensities, to be derived from Eq. (4.17) are equal to the relative areas under the corresponding peaks in the measured diffraction pattern. With explicit results now available it is possible to derive a theoretical expression for the peak areas, from which Eq. (2.66) follows directly. For a monochromatic incident beam the intensity $I_{\Omega}\left(\vartheta_{n}\right)$ at the detector, which is fixed 


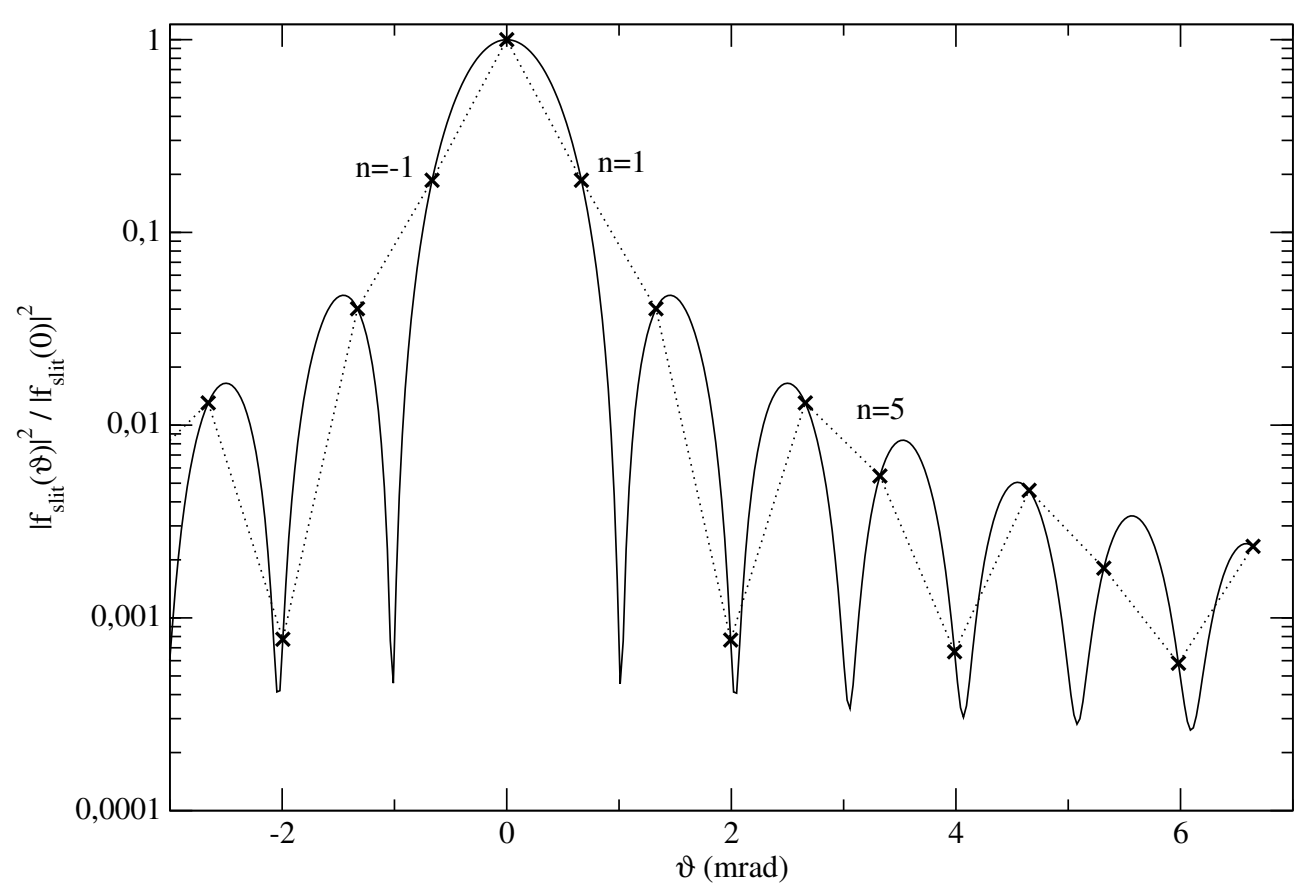

Figure 4.5: The theoretical detection probability of the $n$th principal diffraction order (crosses, joined by dotted lines) are given by the value of the modulus squared of the slit amplitude $f_{\text {slit }}\left(\vartheta_{n}\right)$ (solid line) evaluated at the principal diffraction angles $\vartheta_{n}$ (cf. Eq. (2.63)) of the Fraunhofer theory of diffraction. The slit amplitude contains the atom-bar interaction and is here plotted for a situation with ground-state He atoms at a velocity $v=1800 \frac{\mathrm{m}}{\mathrm{s}}$ which corresponds to a de Broglie wavelength $\lambda=55 \mathrm{pm}$, and a typical grating geometry.

at an angle $\vartheta_{n}$, has been shown to be

$$
I_{\Omega}\left(\vartheta_{n}\right) \propto \int_{\vartheta_{n}-\frac{\Omega}{2}}^{\vartheta_{n}+\frac{\Omega}{2}} \mathrm{~d} \vartheta^{\prime} \delta\left(\vartheta^{\prime}-\vartheta_{n}\right)\left|f_{\text {slit }}\left(\vartheta^{\prime}\right)\right|^{2}=\left|f_{\text {slit }}\left(\vartheta_{n}\right)\right|^{2}
$$

The detector aperture $\Omega$ is usually smaller than the spacing $\vartheta_{1}=\frac{\lambda}{d}$ of the equidistant principal maxima. If the detector scans the angular region $a$ with $\Omega<a<\vartheta_{1}$ there will be a finite signal as long as the $\delta$ peak is within the detector aperture so that a rectangle of a height proportional to $\left|f_{\text {slit }}\left(\vartheta_{n}\right)\right|^{2}$ and a width $\Omega$ will appear in the diffraction pattern. The area $A(n)$ under this rectangular signal around 


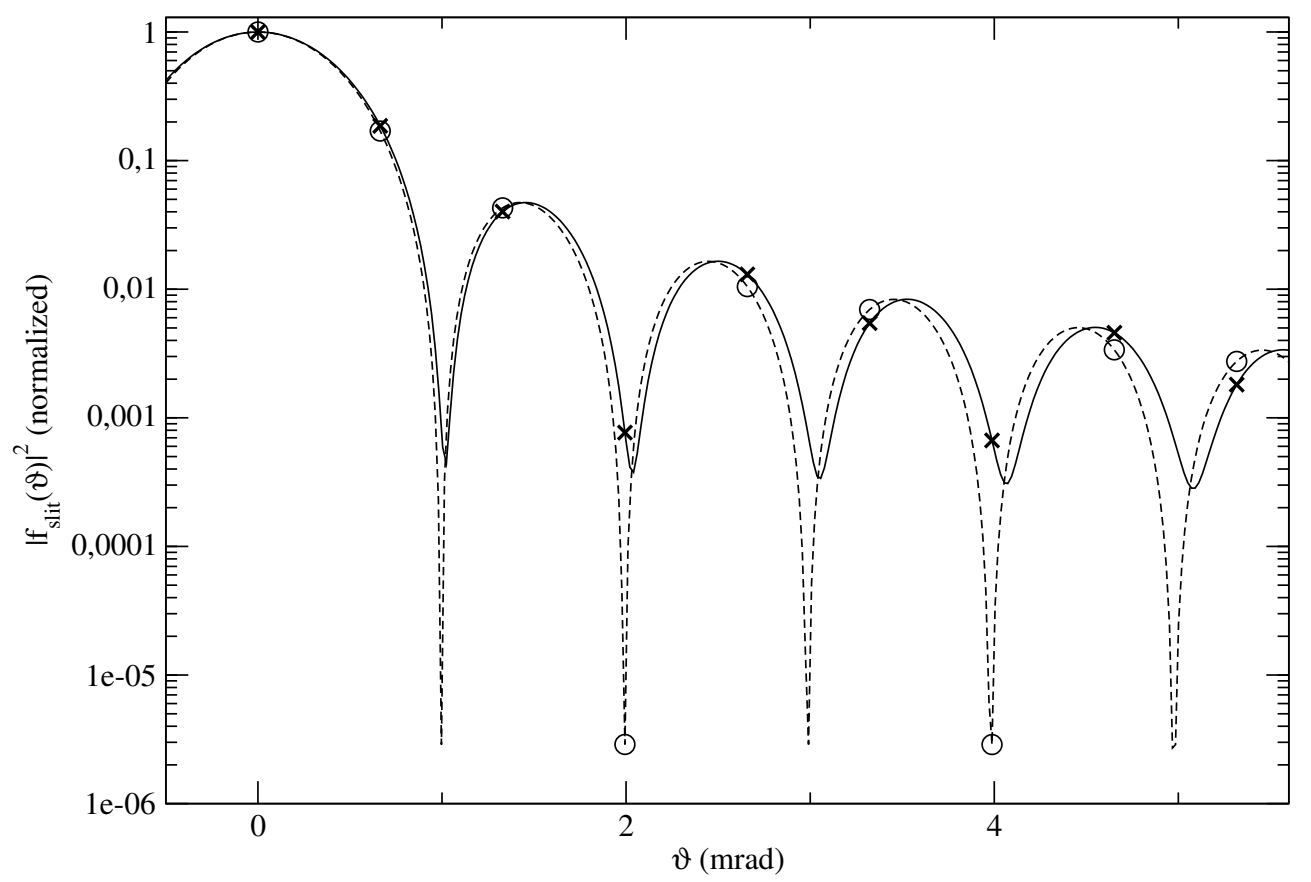

Figure 4.6: The theoretical detection probabilities of the $n$th principal diffraction orders for the cases with atom-bar interaction (crosses) and with only a strongly repulsive core at the bar surface (circles; this case applies to the diffraction of X-rays with a de Broglie wavelength $\lambda=55 \mathrm{pm}$ ) are given by the modulus squared of the respective slit amplitudes $f_{\text {slit }}\left(\vartheta_{n}\right)$ evaluated at the principal diffraction angles $\vartheta_{n}$ in the Fraunhofer limit of diffraction. Due to the atom-bar interaction the slit is effectively narrowed so that the solid line describes a wider slit function than that (dashed line) of the case with only the repulsive core. As the slit functions are evaluated at the same angles the deviations lead to pronounced differences in the detection probabilities.

$\vartheta_{n}$ can be written as

$$
\begin{aligned}
A(n) & =\int_{\vartheta_{n}-\frac{a}{2}}^{\vartheta_{n}+\frac{a}{2}} \mathrm{~d} \vartheta I_{\Omega}(\vartheta) \\
& =\int_{\vartheta_{n}-\frac{a}{2}}^{\vartheta_{n}+\frac{a}{2}} \mathrm{~d} \vartheta \int_{\vartheta_{n}-\frac{\Omega}{2}}^{\vartheta_{n}+\frac{\Omega}{2}} \mathrm{~d} \vartheta^{\prime} \delta\left(\vartheta^{\prime}-\vartheta_{n}\right)\left|f_{\text {slit }}\left(\vartheta^{\prime}\right)\right|^{2} \\
& =\int_{\vartheta_{n}-\frac{a}{2}}^{\vartheta_{n}+\frac{a}{2}} \mathrm{~d} \vartheta \chi_{\left[\vartheta_{n}-\frac{\Omega}{2}, \vartheta_{n}+\frac{\Omega}{2}\right]}(\vartheta)\left|f_{\text {slit }}\left(\vartheta_{n}\right)\right|^{2},
\end{aligned}
$$


with

$$
\chi_{\left[\vartheta_{n}-\frac{\Omega}{2}, \vartheta_{n}+\frac{\Omega}{2}\right]}(\vartheta):=\left\{\begin{array}{lll}
1 & : & \vartheta_{n}-\frac{\Omega}{2}<\vartheta<\vartheta_{n}+\frac{\Omega}{2} \\
0 & : & \text { else . }
\end{array},\right.
$$

Hence it follows that

$$
A_{0}(n)=\Omega\left|f_{\text {slit }}\left(\vartheta_{n}\right)\right|^{2}
$$

Consider now the respective distribution functions $\Phi_{\vartheta}\left(\vartheta_{\text {inc }}\right)$ and $\Phi_{v}(v)$ of the incident angles $\vartheta_{\text {inc }}$ and velocities $v$. Without restricting generality they can be assumed to be normalized with

$$
\int_{-\frac{\pi}{2}}^{\frac{\pi}{2}} \mathrm{~d} \vartheta_{\text {inc }} \Phi_{\vartheta}\left(\vartheta_{\text {inc }}\right)=1
$$

and

$$
\int_{0}^{\infty} \mathrm{d} v \Phi_{v}(v)=1
$$

Due to the collimation of the beam in the experiment the distribution of the incident angles $\Phi_{\vartheta}\left(\vartheta_{\text {inc }}\right)$ is taken to be symmetrical about its maximum at $\vartheta_{\text {inc }}=0$ and narrow as compared to the spacing $\vartheta_{1}$ of the principal diffraction orders. For $\vartheta_{\text {inc }} \neq 0$ the slit amplitude becomes

$$
\begin{aligned}
f_{\text {slit }}(\vartheta) & =\int_{-\frac{s}{2}}^{\frac{s}{2}} \mathrm{~d} x^{\prime} \mathrm{e}^{-\mathrm{i} k x^{\prime}\left(\sin \vartheta+\sin \vartheta_{\text {inc }}\right)} \mathrm{e}^{\mathrm{i} \varphi\left(x^{\prime}\right)} \\
& \approx \int_{-\frac{s}{2}}^{\frac{s}{2}} \mathrm{~d} x^{\prime} \mathrm{e}^{-\mathrm{i} k x^{\prime} \sin \left(\vartheta+\vartheta_{\text {inc }}\right)} \mathrm{e}^{\mathrm{i} \varphi\left(x^{\prime}\right)} .
\end{aligned}
$$

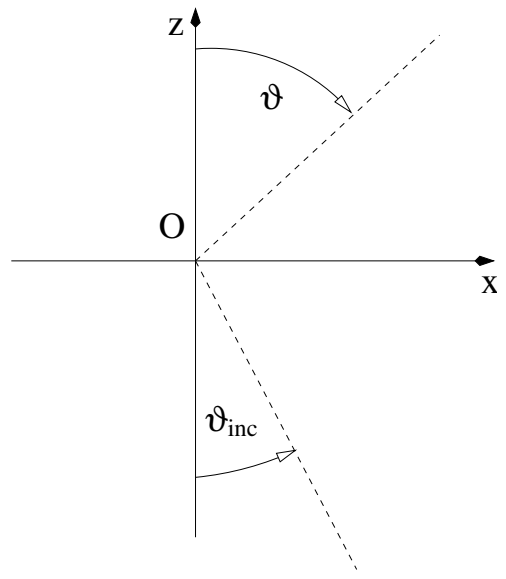

Hereby the angles are defined as indicated in the figure on the left. As all relevant $\vartheta_{\text {inc }}$ are small it is already conceptually included in the eikonal approximation that the atom-bar interaction term $\mathrm{e}^{\mathrm{i} \varphi\left(x^{\prime}\right)}$ in Eq. (4.25) is the same for all incident angles. The grating function $H_{N}(\kappa) \equiv H_{N}(k \sin \vartheta)$ from Eq. (2.60) becomes for $\vartheta_{\text {inc }} \neq 0$

$$
H_{N}\left[k\left(\sin \vartheta+\sin \vartheta_{\mathrm{inc}}\right)\right] \approx H_{N}\left[k \sin \left(\vartheta+\vartheta_{\mathrm{inc}}\right)\right] .
$$

The principal maxima of the grating function are now to be found for arguments of the sine that fulfil $\frac{d k}{2} \sin \left(\vartheta+\vartheta_{\text {inc }}\right) \stackrel{!}{=} n \pi$. In the limit of large numbers $N$ of illuminated slits this yields 


$$
\lim _{N \rightarrow \infty} H_{N}\left[k \sin \left(\vartheta+\vartheta_{\text {inc }}\right)\right] \propto \sum_{n=-\infty}^{+\infty} \delta\left(\vartheta-\vartheta_{\text {inc }}-\vartheta_{n}\right)
$$

With the distribution of incident angles included the area $A_{\vartheta}(n)$ under the detected signal of the $n$th principal maximum becomes

$$
\begin{aligned}
& A_{\vartheta}(n) \propto \int_{\vartheta_{n}-\frac{a}{2}}^{\vartheta_{n}+\frac{a}{2}} \mathrm{~d} \vartheta \int_{\vartheta-\frac{\Omega}{2}}^{\vartheta+\frac{\Omega}{2}} \mathrm{~d} \vartheta^{\prime} \int_{-\frac{\pi}{2}}^{\frac{\pi}{2}} \mathrm{~d} \vartheta_{\text {inc }} \Phi_{\vartheta}\left(\vartheta_{\text {inc }}\right) \delta\left(\vartheta^{\prime}-\vartheta_{\text {inc }}-\vartheta_{n}\right)\left|f_{\text {slit }}\left(\vartheta^{\prime}+\vartheta_{\text {inc }}\right)\right|^{2} \\
& =\int_{\vartheta_{n}-\frac{a}{2}}^{\vartheta_{n}+\frac{a}{2}} \mathrm{~d} \vartheta \int_{-\frac{\pi}{2}}^{\frac{\pi}{2}} \mathrm{~d} \vartheta_{\text {inc }} \Phi_{\vartheta}\left(\vartheta_{\text {inc }}\right) \int_{\vartheta+\vartheta_{\text {inc }}-\frac{\Omega}{2}}^{\vartheta \vartheta_{\text {inc }}+\frac{\Omega}{2}} \mathrm{~d} \vartheta^{\prime} \delta\left(\xi-\vartheta_{n}\right)\left|f_{\text {slit }}(\xi)\right|^{2} \\
& =\int_{-\frac{\pi}{2}}^{\frac{\pi}{2}} \mathrm{~d} \vartheta_{\text {inc }} \Phi_{\vartheta}\left(\vartheta_{\text {inc }}\right) \int_{\vartheta_{n}-\frac{a}{2}}^{\vartheta_{n}+\frac{a}{2}} \mathrm{~d} \vartheta \chi_{\left[\vartheta_{n}+\vartheta_{\text {inc }}-\frac{\Omega}{2}, \vartheta_{n}+\vartheta_{\text {inc }}+\frac{\Omega}{2}\right]}(\vartheta)\left|f_{\text {slit }}\left(\vartheta_{n}\right)\right|^{2} .
\end{aligned}
$$

As the relevant incident angles are small the rectangular function $\chi$ which is shifted by $\vartheta_{\text {inc }}$ in this result always falls within the range $a$ over which the $n$th maximum is scanned. Therefore the result simplifies to

$$
A_{\vartheta}(n) \propto \int_{-\frac{\pi}{2}}^{\frac{\pi}{2}} \mathrm{~d} \vartheta_{\text {inc }} \Phi_{\vartheta}\left(\vartheta_{\text {inc }}\right) \Omega\left|f_{\text {slit }}\left(\vartheta_{n}\right)\right|^{2}=\Omega\left|f_{\text {slit }}\left(\vartheta_{n}\right)\right|^{2} .
$$

As long as only relative areas are considered this result is equivalent to the area $A(n)$ from Eq. (4.20) for a monochromatic beam with $\vartheta_{\text {inc }}=0$.

In view of certain tested qualities of the beam source in the experiment one may assume for the distribution of incident velocities $\Phi_{v}(v)$ that it is symmetrically centred around the most probable value $v_{0}$, and that it is narrow such as to ensure that all relevant atoms have a velocity $v$ which differs from $v_{0}$ by at most $10 \%$. The peak area $A_{\vartheta v}(n)$ of the $n$th order in the diffraction pattern including the incident angle- and velocity distributions can be written as

$$
A_{\vartheta v}(n) \propto \int_{0}^{\infty} \mathrm{d} v \Phi_{v}(v) A_{\vartheta}(n)=\int_{0}^{\infty} \mathrm{d} v \Phi_{v}(v) \Omega\left|f_{\text {slit }}\left(v, \vartheta_{n}\right)\right|^{2}
$$

Analysing the $v$-dependence of $f_{\text {slit }}\left(\vartheta_{n}\right)$ in Eq. (4.25) one notes that $k \sin \vartheta_{n} \equiv$ $\kappa_{n}=\frac{2 \pi n}{d}$ does not depend on the velocity $v$. By the factor $\mathrm{e}^{\mathrm{i} \varphi(x)}$ the slit amplitude depends weakly on $v$ as the phase $\varphi(x) \propto \frac{1}{v}$. For narrow velocity distributions as 
those considered here the incoherent superposition

$$
\int_{0}^{\infty} \mathrm{d} v \Phi_{v}(v)\left|f_{\text {slit }}\left(v, \vartheta_{n}\right)\right|^{2}
$$

is equal to $\left|f_{\text {slit }}\left(v_{0}, \vartheta_{n}\right)\right|^{2}$ within about $0.1 \%$, so the $v$ dependence of $f_{\text {slit }}\left(\vartheta_{n}\right)$ has no effect altogether. As a result, the distribution of incident angles and velocities is expected to have no influence on the relative peak areas and hence

$$
A_{\vartheta v}(n) \propto A_{\vartheta}(n) \propto A(n) \propto\left|f_{\text {slit }}\left(\vartheta_{n}\right)\right|^{2}
$$

For example, the ratio of experimental peak areas $\frac{A_{\exp }(n)}{A_{\exp }(0)}$ directly corresponds to the just derived $\frac{\left|f_{\text {slit }}\left(\vartheta_{n}\right)\right|^{2}}{\left|f_{\text {slit }}(0)\right|^{2}}$ which is equivalent to the equation Eq. $(2.66)$ to be proved.

\subsection{Inclined Incidence}

From an experimentalist's point of view it is not always easy to make sure the beam impinges on the grating perpendicularly. As space inside the evacuated apparatus is limited one may omit the fine positioning devices on behalf of more essential items of machinery. This can lead to deviations from the ideal perpendicular grating set-up of the order of a few angular degrees. While a forward or backward tilt of the grating is unimportant, a rotation around the vertical axis leads to a different phase shift for trajectories in the right and left half of each grating slit which has measurable consequences. A generalization of the result Eq. (4.14) to include the inclined incidence of the atomic beam is therefore required. From a theoretical viewpoint this generalization is a fit goal as much more information about the scattering potential can be revealed in the diffraction picture if the orientation of the target is controlled during the experiment. In the following sections it will be shown that arbitrary incident angles can be taken account of by minor modifications to the results just obtained.

\subsubsection{Bar Potential for Rotated Grating}

As a preparing step one notices that the result Eq. (4.14) can be applied to asymmetrical trapezoids by simply replacing the wedge angle $\beta$ in the first and second term by new angles for the left and right side of the bar, respectively (cf. Fig.4.1).

The situation of inclined incidence is equivalent to that of straight incidence with the grating rotated by an angle $\tau$ with respect to the $x$-axis. It is difficult to 


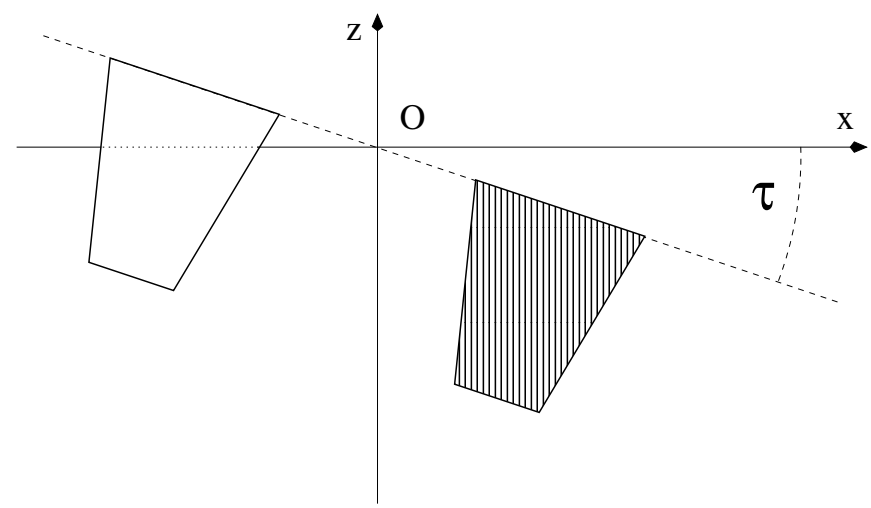

Figure 4.7: An atomic beam along the $z$-axis falls upon a grating that is rotated by an angle $\tau<0$. The area of the grating bars is divided up into thin stripes (as shown on the right bar) that are oriented parallel to the incident beam, i.e. the $z$ axis.

calculate the phase shift $\varphi(x)$ arising from the atom-bar potential like in Eq. (4.14) when the trajectory over which to integrate is no longer perpendicular to the base line of the trapezoid. Additional unwieldy integration limits appear that considerably increase the analytical effort. A simple geometrical consideration can overcome these difficulties.

One may imagine the trapezoid that represents a grating bar as composed of many thin stripes each of which is oriented parallel to the direction of the incident beam. Fig 4.7 shows a sketch of this situation. The calculation of $\varphi$ is not affected by this as the integration over the bar will just sum up all of the stripes. Now looking closer at the structure of the four integrals appearing in Eq. (4.14) one finds that a translation of the potential parallel to the z-axis, i.e. the beam trajectory, does not change the value of $\varphi$, because the integration over $z$ ranges from $-\infty$ to $+\infty$. Therefore it is possible to slide each of the stripes of the trapezoid along in $z$ direction in order to construct an equivalent bar shape whose base line is again perpendicular to the incident beam and that is thus easier to integrate. The boundary conditions of the construction process are that the area of the bar cross-section remain constant and that the distance from the beam trajectory to each area element stay the same which are both guaranteed within the decomposition into stripes.

In a detailed geometrical analysis of the grating rotation $\tau$ there are five cases to be distinguished according to the geometry of the trapezoid. They are $0<|\tau|<\beta,|\tau|=\beta, \beta<|\tau|<\Theta_{1},|\tau|=\Theta_{1}$, and $\Theta_{1}<|\tau|<\Theta_{0}$, characterized by the wedge angle $\beta$ and $\Theta_{1}$, which is defined by

$$
\tan \Theta_{1}:=\frac{b-t \tan \beta}{t}
$$

and which marks the case that the bar is rotated until its diagonal is parallel to 

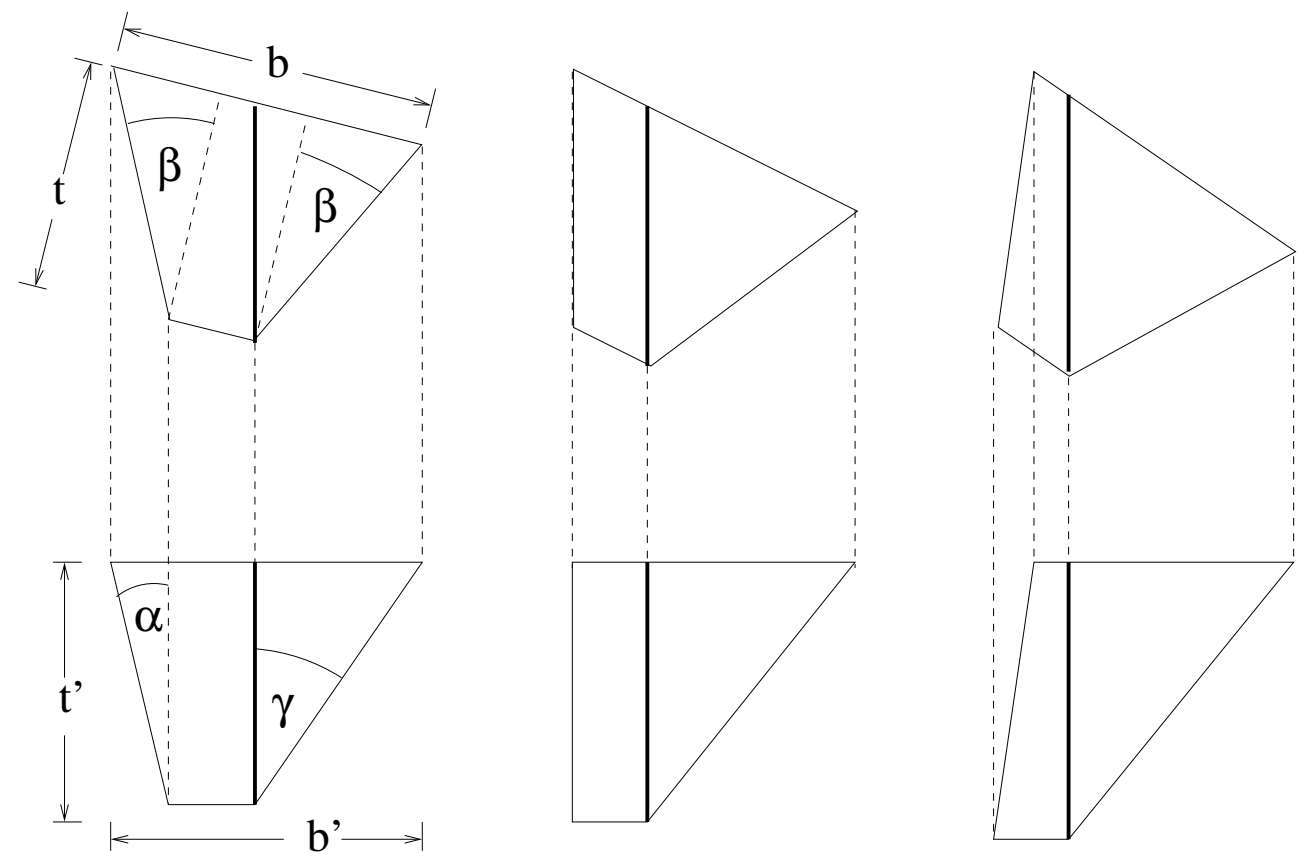

Figure 4.8: Three out of five distinct cases of grating rotation $\tau$. Left: $0<|\tau|<\beta$, middle: $|\tau|=\beta$, right: $\beta<|\tau|<\Theta_{1}$. From the rotated original grating bar crosssections (top row) the shapes equivalent for diffraction experiments are constructed (bottom row) by decomposing the rotated original shape into thin stripes each of which is shifted in $z$ direction (vertically, in the figure) until a new shape is realized whose base line is perpendicular to the $z$ direction. As the length of the stripes does not change the area of each trapezoid is conserved during the operation. An example for one stripe is indicated in the figure by the bold line within each shape.

the incident beam. The maximum rotation angle $\Theta_{0}$ with

$$
\tan \Theta_{0}=\frac{s+t \tan \beta}{t}
$$

is reached when seen in $z$-direction two neighbouring bars begin to overlap so that the transmission through the grating is blocked. To illustrate the construction principle three of the five cases are displayed in Fig. 4.8.

Remarkably, it is possible for any rotation of the original bar with respect to the incident beam to form an equivalent new asymmetrical trapezoid with the face and the base line perpendicular to the incident beam. The new depth $t^{\prime}$, base line $b^{\prime}$, and wedge angles $\alpha$ and $\gamma$ are subject to the same formulae in every case, namely

$$
\begin{aligned}
t^{\prime} & =\frac{t}{\cos \tau}, \\
b^{\prime} & =b \cos \tau,
\end{aligned}
$$




$$
\tan \alpha=\cos ^{2} \tau(\tan \beta+\tan \tau)
$$

and

$$
\tan \gamma=\cos ^{2} \tau(\tan \beta-\tan \tau) .
$$

It should be commented that the limit $\tau \rightarrow \pm \frac{\pi}{2}$ is not relevant for the current application because it makes no sense to have the incident beam parallel to the grating plane and perpendicular to the bars.

\subsubsection{Slit Amplitude for Rotated Grating}

In addition to the modifications of the bar potential as explained in the previous section the features arising from the repulsive potential that have been seen to be closely analogous to classical optics also undergo some changes when the grating is rotated with respect to the incident beam.

Introducing a new coordinate axis $\xi:=\frac{x}{\cos \tau}$ along the rear side of the rotated grating the incident wave which is in the case $\tau=0$ represented by unity under the integral Eq. (4.18) for the slit amplitude becomes $\mathrm{e}^{\mathrm{i} k \xi \sin \tau}$ in order to account for advanced or retarded arrival in sections of the grating tilted towards or away from the source, respectively. Similarly, the geometrical phase factor $\mathrm{e}^{-\mathrm{i} k x \sin \vartheta}$ in Eq. (4.18) is for a rotated grating appropriately replaced by $\mathrm{e}^{-\mathrm{i} k \xi \sin (\vartheta+\tau)}$. With

$$
\Phi(\tau):=\sin (\vartheta+\tau)-\sin \tau
$$

the total geometrical phase factor can be written as $\mathrm{e}^{-\mathrm{i} k \xi \Phi(\tau)}$. Apart from that, for rotation angles $|\tau|>\beta$ the illuminated region within each grating opening is reduced as parts of the incoming beam are blocked out by the adjacent bars. Denoting by $s_{1}$ the left end and by $s_{\mathrm{r}}$ the right end of the illuminated spot, expressed in terms of the new coordinate $\xi$, one has

$$
s_{1}(\tau)=\left\{\begin{array}{rl}
-\frac{s}{2} \quad: \quad-\Theta_{0} \leq \tau \leq \beta \\
t(\tan \tau-\tan \beta)-\frac{s}{2} \quad: \quad \beta \leq \tau \leq \Theta_{0},
\end{array},\right.
$$

and

$$
s_{\mathrm{r}}(\tau)=\left\{\begin{aligned}
\frac{s}{2}+t(\tan \beta+\tan \tau) & :-\Theta_{0} \leq \tau \leq-\beta \\
\frac{s}{2} & :-\beta \leq \tau \leq \Theta_{0}
\end{aligned}\right.
$$

With the essential modifications now complete one obtains for the area $A_{0}(n)$ under the $n$th order maximum in the diffraction pattern

$$
A(n) \propto\left|f_{\text {slit }}\left(\vartheta_{n}, \tau\right)\right|^{2},
$$


with

$$
f_{\text {slit }}\left(\vartheta_{n}, \tau\right):=\int_{s_{1}(\tau)}^{s_{\mathrm{r}}(\tau)} \mathrm{d} \xi \mathrm{e}^{-\mathrm{i} k \xi \sin \Phi(\tau)} \mathrm{e}^{\mathrm{i} \varphi_{\tau}(x)}
$$

With the results Eq. (4.35) $f f$ the phase $\varphi_{\tau}(x)$ for a grating rotated by $\tau$ is written as a sum

$$
\varphi_{\tau}(x)=\varphi_{\tau}^{\mathrm{l}}(x)+\varphi_{\tau}^{\mathrm{r}}(x)
$$

of the contributions $\varphi_{\tau}^{\mathrm{l}}(x)$ and $\varphi_{\tau}^{\mathrm{r}}(x)$ of the left and right bar, respectively. The latter is given by

$$
\begin{aligned}
\varphi_{\tau}^{\mathrm{r}}(x) & =\frac{C_{3}}{2 \hbar v \tan \alpha}\left\{\frac{1}{\zeta_{\mathrm{r}}^{2}}-\frac{1}{\left(\zeta_{\mathrm{r}}+t^{\prime} \tan \alpha\right)^{2}}\right\}+ \\
& +\frac{C_{3}}{2 \hbar v \tan \gamma}\left\{\frac{1}{\left(\zeta_{\mathrm{r}}+b^{\prime}\right)^{2}}-\frac{1}{\left(\zeta_{\mathrm{r}}+b^{\prime}-t^{\prime} \tan \gamma\right)^{2}}\right\}- \\
& -\frac{C_{9}}{8 \hbar v \tan \alpha}\left\{\frac{1}{\zeta_{\mathrm{r}}^{8}}-\frac{1}{\left(\zeta_{\mathrm{r}}+t^{\prime} \tan \alpha\right)^{8}}\right\}+ \\
& +\frac{C_{9}}{8 \hbar v \tan \gamma}\left\{\frac{1}{\left(\zeta_{\mathrm{r}}+b^{\prime}\right)^{8}}-\frac{1}{\left(\zeta_{\mathrm{r}}+b^{\prime}-t^{\prime} \tan \gamma\right)^{8}}\right\},
\end{aligned}
$$

with the modified impact parameter

$$
\zeta_{\mathrm{r}}:=\cos \tau s_{\mathrm{r}}(\tau)-x \equiv \cos \tau\left[s_{\mathrm{r}}(\tau)-\xi\right]
$$

that describes the least distance between the trajectory and the rotated grating bar. Note that for rotation angles $\tau$ greater than the wedge angle $\beta$ of the original bar the leading edge of the bar is closer to the trajectory than the trailing edge. A sketch of the situation is presented in Fig. 4.9. The phase contribution of the bar to the left of the slit is given by

$$
\begin{aligned}
\varphi_{\tau}^{1}(x) & =\frac{C_{3}}{2 \hbar v \tan \gamma}\left\{\frac{1}{\zeta_{1}^{2}}-\frac{1}{\left(\zeta_{1}+t^{\prime} \tan \gamma\right)^{2}}\right\}+ \\
& +\frac{C_{3}}{2 \hbar v \tan \alpha}\left\{\frac{1}{\left(\zeta_{1}+b^{\prime}\right)^{2}}-\frac{1}{\left(\zeta_{1}+b^{\prime}-t^{\prime} \tan \alpha\right)^{2}}\right\}- \\
& -\frac{C_{9}}{8 \hbar v \tan \gamma}\left\{\frac{1}{\zeta_{1}^{8}}-\frac{1}{\left(\zeta_{1}+t^{\prime} \tan \gamma\right)^{8}}\right\}+ \\
& +\frac{C_{9}}{8 \hbar v \tan \alpha}\left\{\frac{1}{\left(\zeta_{1}+b^{\prime}\right)^{8}}-\frac{1}{\left(\zeta_{1}+b^{\prime}-t^{\prime} \tan \alpha\right)^{8}}\right\},
\end{aligned}
$$

with the corresponding impact parameter

$$
\zeta_{1}:=x-\cos \tau s_{1}(\tau) \equiv \cos \tau\left[\xi-s_{1}(\tau)\right] .
$$




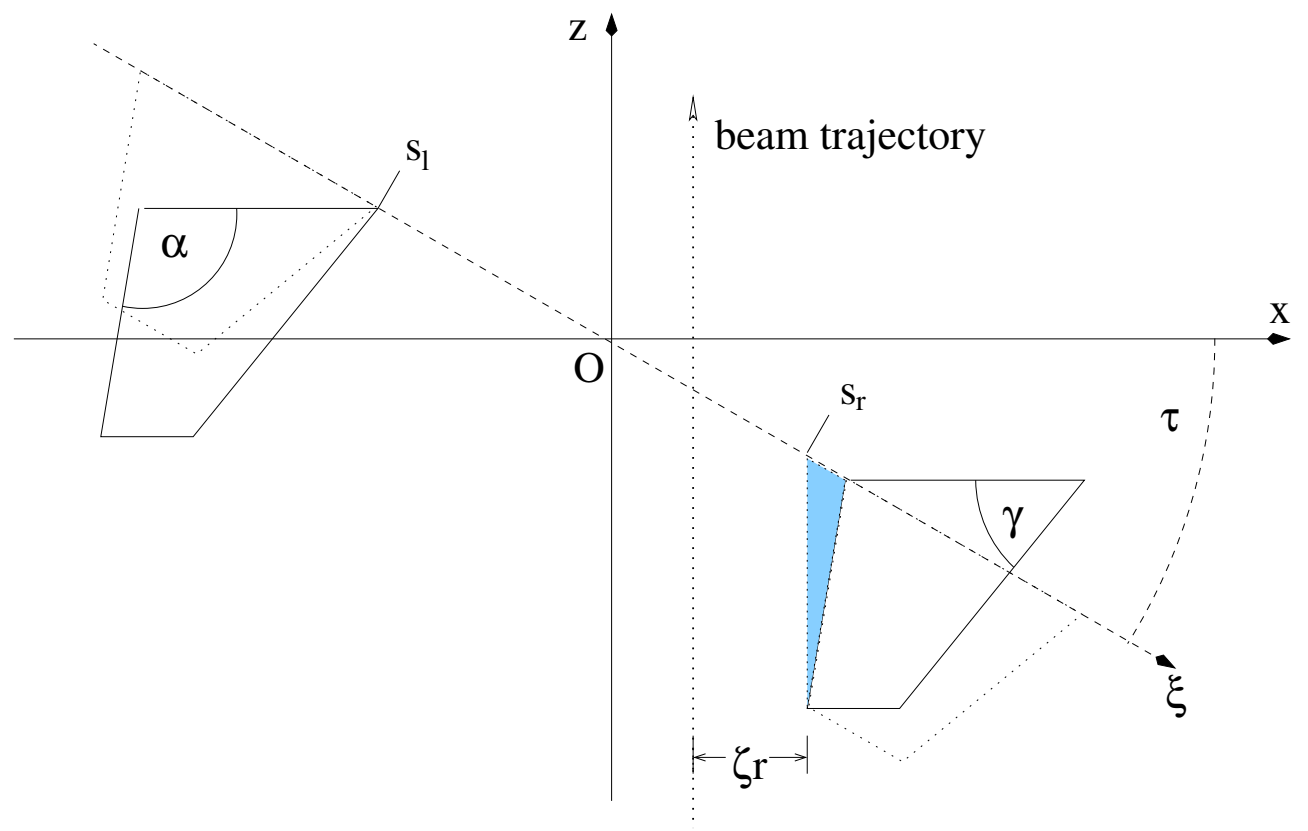

Figure 4.9: For a grating rotation $\tau<0$ the slit amplitude is given by an integral along the $\xi$ axis from $s_{1}$ to $s_{\mathrm{r}}$. The latter boundary takes account of the "shadow" (shaded area) cast by the leading edge of the right bar. The impact parameter $\zeta_{\mathrm{r}}$ is defined as the least distance between the beam trajectory and the right bar. The dotted lines show the original bar shapes while the two identical shapes framed by solid lines are the constructed equivalent bar cross-sections that are easier to integrate. The modified wedge angles are indicated in the figure.

The grating rotation causes an interesting effect of asymmetry in the intensity pattern which clearly distinguishes atom diffraction from optics. From Fig. 4.9 it can be seen that for rotation angles $\tau<0$ the beam trajectories are more parallel to the side of the right bar than to that of the bar on the left of the slit. As a consequence, the atom-bar interaction is stronger on the right-hand-side of the slit which in the diffraction pattern leads to an enveloping slit amplitude function which is wider on the side where the interaction is stronger. This coincides with the classical notion of more atoms being attracted towards the right bar and thus being deflected to larger angles. Fig.4.10 illustrates the phenomenon with the help of a theoretical example with metastable helium atoms at $v=1500 \frac{\mathrm{m}}{\mathrm{s}}$ diffracted from a grating whose bars have a wedge angle $\beta=11^{\circ}$. Besides the asymmetry of the the slit amplitude function there are two well-known effects apparent in the figure. First, the slit amplitude function is widened on both sides of the diffraction pattern upon grating rotation because the slit is effectively narrowed by a factor $\cos \tau$. Second, the principal diffraction angles increase because upon the rotation $\tau \neq 0$ the grating function $H_{N}$ (cf. Eq. (2.60)) assumes 


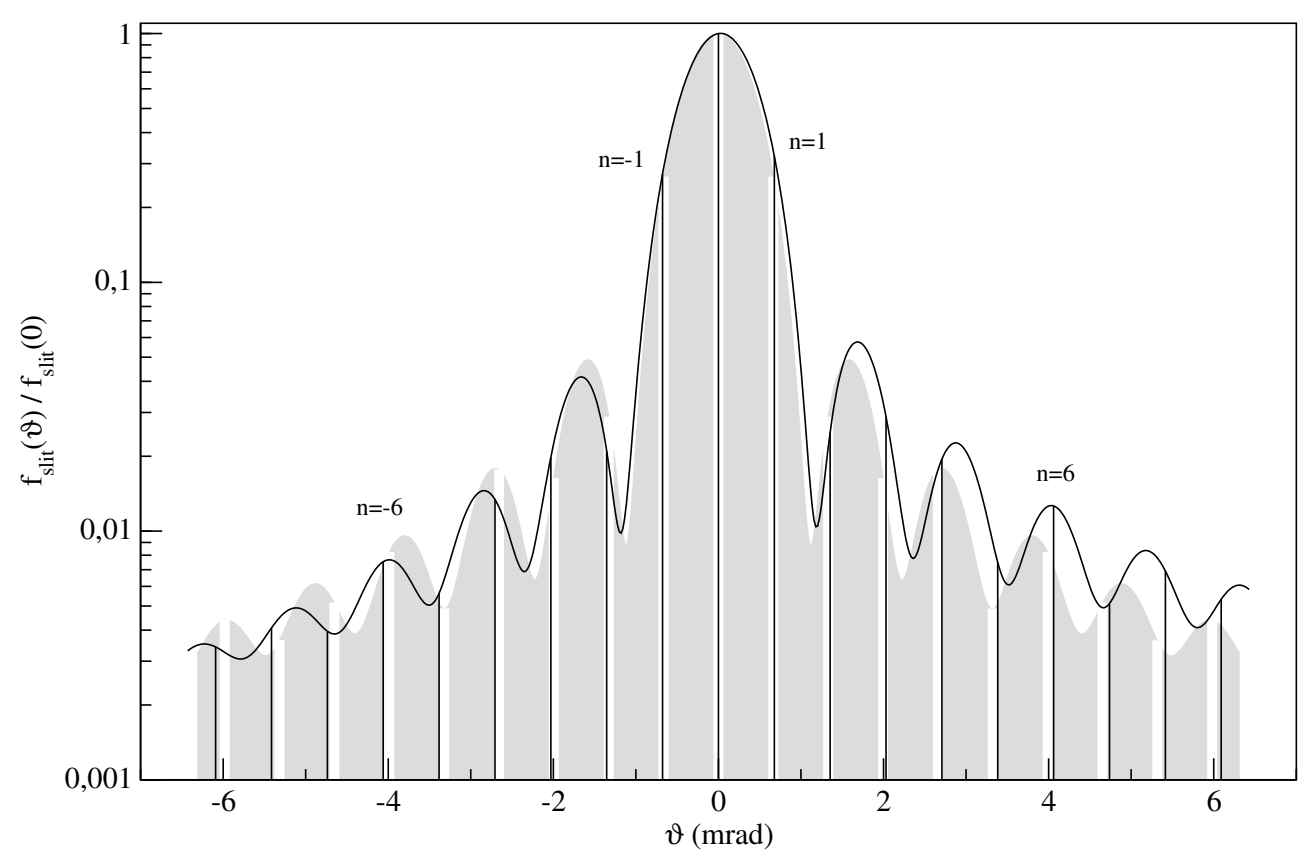

Figure 4.10: Theoretical diffraction pattern for a beam of metastable helium atoms and a transmission grating whose bars have a wedge angle $\beta=11^{\circ}$. The shaded curve is the enveloping slit amplitude function for the case $\tau=0$, i.e. no grating rotation. White bars are added whose centres mark the principal diffraction angles. For a grating rotation of $\tau=-10.9^{\circ}$ the envelope function (solid line) assumes higher values for positive diffraction angles $\vartheta$. This is due to different atom-bar interaction potentials on the sides of each grating slit. The envelope function is generally wider for $\tau \neq 0$ because the rotated grating slits appear more narrow when seen in beam direction. For increasing diffraction orders $n$ the vertical solid lines at the principal diffraction angles for the rotated grating are seen to wander out of the white bars whose centres mark the principal angles in the non-rotated case, i.e. the principal orders assume a wider spacing for rotated gratings. This well-known optical effect does also apply to the diffraction of atoms (see Eq. (4.50)).

its principal maxima under the condition

$$
\sin \left[\frac{k d \Phi(\tau)}{2}\right] \stackrel{!}{=} 0 \quad \Leftrightarrow \quad \Phi(\tau) \equiv \sin (\vartheta+\tau)-\sin \tau \stackrel{!}{=} \frac{n \lambda}{d} \equiv \vartheta_{n} .
$$

In view of the detector angles $\vartheta$ being as small as a few mrad a first-order Taylor expansion may be used to obtain

$$
\vartheta=\frac{\vartheta_{n}}{\cos \tau}
$$

which states that the principal diffraction angles upon grating rotation are equidistant as in the case $\tau=0$, but the spacing increases by a factor $\frac{1}{\cos \tau}$. 


\subsection{Surface Roughness}

The theory presented so far has been based on an idealized shape of the grating bars, namely that of a trapezoidal prism. From scanning electron micrographs like Fig. 4.2 it is evident that the real grating bars exhibit certain deviations from the idealized shape. These corrugations of the bar faces are due to the manufacturing method of the gratings which includes an etching process to produce the slits.

For the diffraction experiments, only the corrugation of the bar sides is relevant while that of the front and rear faces can be ignored. The particular aspect of the bar side corrugation that leads to a deviation of the bar cross-section from an ideal trapezoid will be treated in detail in section 5.1 .3 of this work.

In the following, the other important consequence of the bar side corrugation is discussed that leads to wavy rather than straight trailing edges of the grating bars so that the grating slits are in fact bounded by undulated lines. Of course, this situation requires a three-dimensional approach that includes the $y$ direction along which the bar edges are undulated. This approach, however, is analogous to the two-dimensional case, particularly with respect to the eikonal approximation, and apart from constant factors. For the interpretation of experimental data only relative diffraction intensities are relevant so that those constant factors can be left out of the consideration.

The three dimensional wavefunction $\tilde{\psi}(\tilde{\mathbf{r}})$ at the detector slit position $\tilde{\mathbf{r}}$ is then given by

$$
\tilde{\psi}(\tilde{\mathbf{r}}) \propto \sum_{j} \int_{-\frac{Y}{2}}^{\frac{Y}{2}} \mathrm{~d} y^{\prime} \int_{-\frac{s}{2}-\Delta_{j}^{1}\left(y^{\prime}\right)}^{\frac{s}{2}-\Delta_{j}^{\mathrm{r}}\left(y^{\prime}\right)} \mathrm{d} x^{\prime} \mathrm{e}^{\mathrm{i} k \sqrt{\left(x-x^{\prime}-j d\right)^{2}+\left(y-y^{\prime}\right)^{2}+z^{2}}} \tilde{\psi}\left(x^{\prime}+j d, y^{\prime}, 0\right),
$$

where $\Delta_{j}^{1}\left(y^{\prime}\right)$ and $\Delta_{j}^{\mathrm{r}}\left(y^{\prime}\right)$ stand for the difference between the respective left and right boundaries of the $j$ th grating slit, taken at a vertical position $y^{\prime}$, and their respective mean values $\pm \frac{s}{2}+j d$ with respect to an average over the height $Y$ of the grating. In view of the interferometric technique that is employed to write the slits on the silicon nitride substrate during the manufacturing process it is improbable and contrary to the assertions of the manufacturers that the periodicity of the slits be subject to a systematical error, especially since the gratings have been tested to maintain their periodicity well over distances a hundred times longer than the typical width of the illuminated region on the grating in the experiment.

On expansion of the square root in Eq. (4.51) one obtains in first order

$$
\sqrt{\left(x-x^{\prime}-j d\right)^{2}+\left(y-y^{\prime}\right)^{2}+z^{2}} \approx \tilde{r}-\frac{x}{\tilde{r}}\left(x^{\prime}+j d\right)+\frac{\left(x^{\prime}+j d\right)^{2}}{2 \tilde{r}}+\frac{\left(y-y^{\prime}\right)^{2}}{2 \tilde{r}},
$$


from which it can be seen that within this approximation the integrand in Eq. 4.51) contains a factor $\mathrm{e}^{i \frac{\left(y-y^{\prime}\right)^{2}}{2 \tilde{r}}}$.

The wavefunction $\tilde{\psi}\left(x^{\prime}+j d, y^{\prime}, 0\right)$ which also appears in the integrand in Eq. (4.51) is well collimated with respect to the $x^{\prime}$ direction because the collimation slits in the experiment are sufficiently narrow to produce a coherent wavefront on the grating over about 100 periods (see section 5.4.2). The coherence length $L_{y}$ in the vertical $y$ direction can be estimated from the width of the central Fraunhofer diffraction maximum as results from the vertical diffraction of atoms at a typical de Broglie wavelength $\lambda \approx 50 \mathrm{pm}$ from one of the $5 \mathrm{~mm}$-high collimation slits. In analogy with classical optics the amplitude $A(\theta)$ diffracted at a vertical angle $\theta$ is given by

$$
A(\theta) \propto \frac{\sin \left(\frac{k h}{2} \sin \theta\right)}{k \sin \theta},
$$

where $h$ stands for the height of the collimation slit. For a distance $\tilde{r} \approx 1 \mathrm{~m}$ between the collimator and the grating the $\operatorname{sine} \sin (\theta)$ of the diffraction angle $\theta$ can be replaced by $\frac{y^{\prime}}{\tilde{r}}$, where $y^{\prime}$ is the vertical coordinate on the grating. The coherence length $L_{y}$ is then estimated by

$$
\frac{k h}{2} \frac{L_{y}}{\tilde{r}}=\pi
$$

from which follows $L_{y} \approx 10 \mathrm{~nm}$ for typical values of $k \equiv \frac{2 \pi}{\lambda}$. This result indicates that in Eq. (4.51) it is sufficient to describe the diffraction of an atom by an integral in $y^{\prime}$ direction that runs over the coherence length $L_{y}$ rather than the total illuminated height $Y$ of the grating. Within these limited boundaries the factor $\mathrm{e}^{i \frac{\left(y-y^{\prime}\right)^{2}}{2 \tilde{r}}}$ can be replaced by unity because

$$
k \frac{\left(y-y^{\prime}\right)^{2}}{2 \tilde{r}} \leq \frac{k}{2 \tilde{r}} L_{y}^{2} \approx 1.510^{-3}
$$

For a detector slit position $\tilde{\mathbf{r}}$ in the $x-z$ plane the amplitude becomes

$$
\tilde{\psi}(\tilde{\mathbf{r}}) \propto \sum_{j} \int_{-\frac{L_{y}}{2}}^{\frac{L_{y}}{2}} \mathrm{~d} y^{\prime} \int_{-\frac{s}{2}-\Delta_{j}^{1}\left(y^{\prime}\right)}^{\frac{s}{2}+\Delta_{j}^{\mathrm{r}}\left(y^{\prime}\right)} \mathrm{d} x^{\prime} \mathrm{e}^{-\mathrm{i} \kappa\left(x^{\prime}+j d\right)} \tilde{\psi}\left(x^{\prime}+j d, y^{\prime}, 0\right),
$$

where the Fraunhofer limit with $\kappa:=k \sin (\vartheta)$ has been adopted for horizontal diffraction angles $\vartheta$. This approximation is sufficient to reveal the essential influence of the edge roughness on the detected intensity. In the real experiment, the detector slit position is not specified with respect to the vertical coordinate $y$ as the detector slit is $5 \mathrm{~mm}$ high. Because of the small coherence length $L_{y}$ the vertical coordinate $y$ of where the particle enters the detector slit is determined 
by the vertical position $y^{\prime} \pm \frac{L_{y}}{2}$ of where the particle passes through the grating. Therefore the height of the detector slit does not lead to any new effects of coherence, it merely helps to increase the count rate in the experiment.

For a given trajectory that passes through the grating at $\left(x^{\prime}+j d, y^{\prime}, 0\right)^{\mathrm{T}}$ the wavefunction $\tilde{\psi}\left(x^{\prime}+j d, y^{\prime}, 0\right)$ in the integrand of Eq. 4.56$)$ is given by

$$
\tilde{\psi}\left(x^{\prime}+j d, y^{\prime}, 0\right)=\mathrm{e}^{\mathrm{i} \tilde{\varphi}\left(x^{\prime}+j d, y^{\prime}\right)} .
$$

This expression describes a plane wave arriving at the rear side plane $z^{\prime}=0$ of the grating inside the slits, which carries the additional phase $\tilde{\varphi}$ due to the interaction of the atom with the grating bars. For the current purpose the crosssection of the bars is taken to be a trapezoidal prism whose width $b$ varies with the vertical coordinate $y^{\prime}$ like

$$
b_{j}\left(y^{\prime}\right)=d-s-\Delta_{j}^{1}\left(y^{\prime}\right)-\Delta_{j-1}^{\mathrm{r}}\left(y^{\prime}\right)
$$

which leads to an undulated trailing bar edge as intended to discuss now. The so assumed theoretical bar shape is illustrated in Fig. 4.11; it is conceptually close to the real bar shape as can be seen from a comparison with Fig. 4.2.

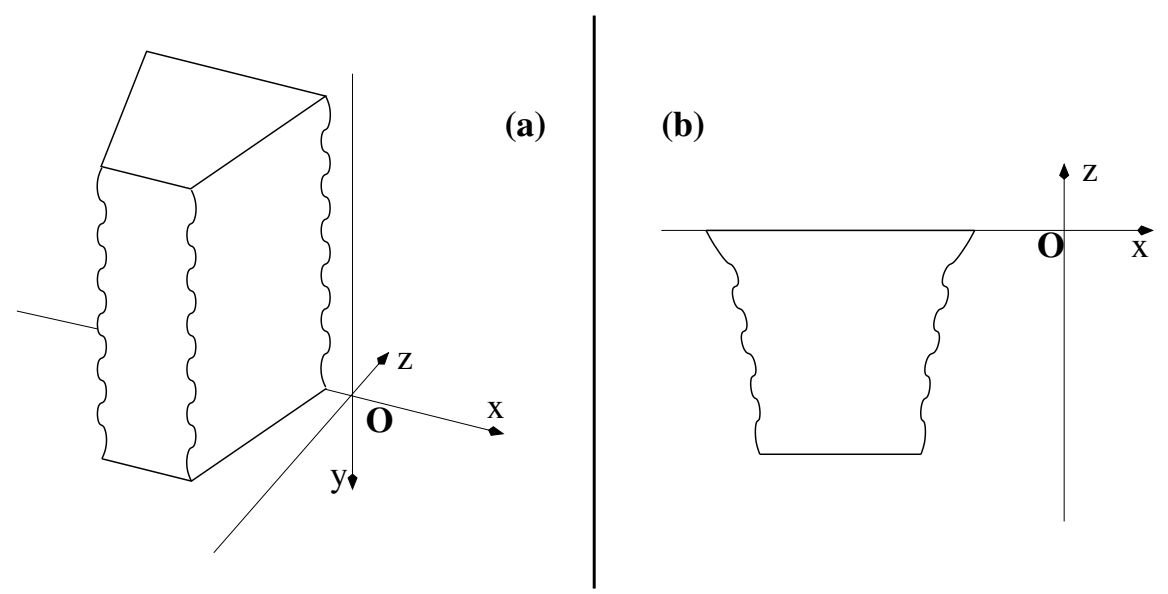

Figure 4.11: (a): Model three-dimensional shape of a grating bar that includes the feature of edge roughness. The chosen model is close to the form of the real grating bars as can be seen from the scanning electron micrograph Fig.4.2. (b): The corrugation of the bar sides that leads to a deviation of the bar cross-section from an ideal trapezoid has a qualitatively different influence on the diffraction of atoms. It is excluded from the current investigation and is treated in section 5.1.3.

The phase $\tilde{\varphi}$ that appropriately accounts for the three-dimensional grating bar shape is written, similarly to Eq. (4.8), as

$$
\tilde{\varphi}\left(x^{\prime}+j d, y^{\prime}\right)=\tilde{\varphi}_{1}\left(x^{\prime}+j d, y^{\prime}\right)+\tilde{\varphi}_{\mathrm{r}}\left(x^{\prime}+j d, y^{\prime}\right),
$$


with

$\tilde{\varphi}_{1}(x+j d, y)=\frac{6 C_{3}}{\pi \hbar v} \int_{-\infty}^{+\infty} \mathrm{d} z \int_{-t}^{0} \mathrm{~d} z^{\prime} \int_{-\infty}^{+\infty} \mathrm{d} y^{\prime} \int_{-\frac{s}{2}-\Delta_{j}^{1}\left(y^{\prime}\right)-b_{j}\left(y^{\prime}\right)-z^{\prime} \tan \beta}^{-\frac{s}{2}-\Delta_{j}^{1}\left(y^{\prime}\right)+z^{\prime} \tan \beta} \mathrm{d} x^{\prime}\left[\left(x-x^{\prime}\right)^{2}+\left(y-y^{\prime}\right)^{2}+\left(z-z^{\prime}\right)^{2}\right]^{-3}$

and

$\tilde{\varphi}_{\mathrm{r}}(x+j d, y)=\frac{6 C_{3}}{\pi \hbar v} \int_{-\infty}^{+\infty} \mathrm{d} z \int_{-t}^{0} \mathrm{~d} z^{\prime} \int_{-\infty}^{+\infty} \mathrm{d} y^{\prime} \int_{\frac{s}{2}+\Delta_{j}^{\mathrm{r}}\left(y^{\prime}\right)-z^{\prime} \tan \beta}^{\frac{s}{2}+\Delta_{j}^{\mathrm{r}}\left(y^{\prime}\right)+b_{j+1}\left(y^{\prime}\right)+z^{\prime} \tan \beta} \mathrm{d} x^{\prime}\left[\left(x-x^{\prime}\right)^{2}+\left(y-y^{\prime}\right)^{2}+\left(z-z^{\prime}\right)^{2}\right]^{-3}$.

Note that the coordinates of the beam trajectory that appear in the argument on the left-hand-side of the last two equations have been changed in order to avoid doubly primed quantities under the integral. $x$ and $y$ now stand for the position of the beam trajectory while the primed quantities locate volume elements inside the bars. For the sake of simplicity at this point the terms proportional to $C_{9}$, that in Eq. (4.14) and account for the detailed shape of the repulsive atom-bar potential, have been omitted in Eq. (4.60) and Eq. (4.61). Their inclusion is accomplished analogously to the following procedure. In contrast to Eq. (4.14), Eq. (4.60) and Eq. (4.61) cannot readily be solved because the integral over $y^{\prime}$ is no longer trivial due to the presence of the edge roughness that depends on $y^{\prime}$ which, in principal, requires the calculation of the dispersion potential of each point of a given trajectory with respect to each point on the corrugated surface, no matter how distant and how feeble its influence may be. An obvious simplification in order to proceed is to expand Eq. (4.60) and Eq. (4.61) with respect to small $y^{\prime}$ and retain only the lowest order. This means that for each $y$ the region of the edge that significantly contributes to the phase $\tilde{\varphi}$ is assumed to be locally straight. For a typical range of the dispersion interaction of a few nanometers and the observed corrugation lengths of about the same size this approximation is roughly justified. It is important to note that the edge roughness is not thrown away with this step but still present through the edge positions characterised by $\Delta_{j}^{\mathrm{r}}\left(y^{\prime}\right)$, etc. For example, Eq. (4.61) becomes within this approximation

$\tilde{\varphi}_{\mathrm{r}}(x+j d, y)=\frac{6 C_{3}}{\pi \hbar v} \int_{-\infty}^{+\infty} \mathrm{d} z \int_{-t}^{0} \mathrm{~d} z^{\prime} \int_{-\infty}^{+\infty} \mathrm{d} y^{\prime} \int_{\frac{s}{2}+\Delta_{j}^{\mathrm{r}}(y)-z^{\prime} \tan \beta}^{\frac{s}{2}+\Delta_{j}^{\mathrm{r}}(y)+b_{j+1}(y)+z^{\prime} \tan \beta} \mathrm{d} x^{\prime}\left[\left(x-x^{\prime}\right)^{2}+\left(y^{\prime}\right)^{2}+\left(z-z^{\prime}\right)^{2}\right]^{-3}$,

whereby the left and right boundaries of the right grating bar are evaluated only at $y$ which is the vertical position at which the particle passes through the grating. 
The integrals can be performed like in Eq. (4.14) which yields

$$
\begin{aligned}
& \tilde{\varphi}_{\mathrm{r}}(x+j d, y)=\frac{C_{3}}{2 \hbar v \tan \beta}\left\{\frac{1}{\zeta^{2}}-\frac{1}{(\zeta+t \tan \beta)^{2}} \quad+\right. \\
& \left.+\frac{1}{\left(\zeta+b_{j+1}(y)\right)^{2}}-\frac{1}{\left(\zeta+b_{j+1}(y)-t \tan \beta\right)^{2}}\right\}
\end{aligned}
$$

where the impact parameter $\zeta:=\frac{s}{2}+\Delta_{j}^{\mathrm{r}}(y)-x$ measures the horizontal distance from an atomic trajectory at $(x+j d, y, 0)^{\mathrm{T}}$ to the corrugated edge of the bar on the right-hand-side of the $j$ th slit. As for the moment grating rotations are not considered one may neglect the terms in Eq. (4.63) that contain the width $b_{j+1}(y)$ of the bar. This simplification included, the phase $\tilde{\varphi}_{1}(x+j d, y)$ arising from the interaction of the atoms with the bar on the left side of the $j$ th slit becomes

$$
\tilde{\varphi}_{1}(x+j d, y)=\frac{C_{3}}{2 \hbar v \tan \beta}\left\{\frac{1}{\zeta^{2}}-\frac{1}{(\zeta+t \tan \beta)^{2}}\right\} .
$$

Here, $\zeta$ stands for $x+\frac{s}{2}+\Delta_{j}^{1}(y)$ which is the distance between the atomic trajectory and the corrugated edge of the left bar.

Formally inserting the results Eq. (4.63) and Eq. (4.64) into Eq. (4.56) one obtains

$$
\begin{aligned}
\tilde{\psi}(\tilde{\mathbf{r}}) \propto & \sum_{j} \mathrm{e}^{-\mathrm{i} \kappa j d} \int_{-\frac{L_{y}}{2}}^{\frac{L_{y}}{2}} \mathrm{~d} y^{\prime}\left\{\int_{-\frac{s}{2}-\Delta_{j}^{1}\left(y^{\prime}\right)}^{0} \mathrm{~d} x^{\prime} \mathrm{e}^{-\mathrm{i} \kappa x^{\prime}} \mathrm{e}^{\mathrm{i}\left[\tilde{\varphi}_{1}\left(x^{\prime}+j d, y\right)+\tilde{\varphi}_{\mathrm{r}}\left(x^{\prime}+j d, y\right)\right]}+\right. \\
+ & \int_{0}^{\frac{s}{2}+\Delta_{j}^{\mathrm{r}}\left(y^{\prime}\right)} \mathrm{d} x^{\prime} \mathrm{e}^{-\mathrm{i} \kappa x^{\prime}} \mathrm{e}^{\mathrm{i}\left[\tilde{\varphi}_{1}\left(x^{\prime}+j d, y\right)+\tilde{\varphi}_{\mathrm{r}}\left(x^{\prime}+j d, y\right)\right]}
\end{aligned}
$$

With the separation of the integral into the respective left and right half of the slit one may for non-rotated gratings drop the phase contribution from the bar of the respective opposite side because it is practically equal to zero. After applying the substitutions $\zeta:=x^{\prime}+\frac{s}{2}+\Delta_{j}^{1}(y)$ to the first integral in Eq. (4.65), and $\zeta:=\frac{s}{2}+\Delta_{j}^{\mathrm{r}}(y)-x^{\prime}$ to the second one finds

$$
\begin{gathered}
\tilde{\psi}(\tilde{\mathbf{r}}) \propto \sum_{j} \mathrm{e}^{-\mathrm{i} \kappa j d} \int_{-\frac{L_{y}}{2}}^{\frac{L_{y}}{2}} \mathrm{~d} y^{\prime}\left\{\int_{0}^{\frac{s}{2}+\Delta_{j}^{1}\left(y^{\prime}\right)} \mathrm{d} \zeta \mathrm{e}^{-\mathrm{i} \kappa\left[\zeta-\frac{s}{2}-\Delta_{j}^{1}(y)\right]} \mathrm{e}^{\mathrm{i} \varphi(\zeta)}+\right. \\
\left.+\int_{0}^{\frac{s}{2}+\Delta_{j}^{\mathrm{r}}\left(y^{\prime}\right)} \mathrm{d} \zeta \mathrm{e}^{\mathrm{i} \kappa\left[\zeta-\frac{s}{2}-\Delta_{j}^{\mathrm{r}}(y)\right]} \mathrm{e}^{\mathrm{i} \varphi(\zeta)}\right\},
\end{gathered}
$$


where the phase

$$
\varphi(\zeta):=\frac{C_{3}}{2 \hbar v \tan \beta}\left\{\frac{1}{\zeta^{2}}-\frac{1}{(\zeta+t \tan \beta)^{2}}\right\}
$$

is the same for both integrals. Since it refers to straight stretches of the bar edges it is equal to the phase used in the two-dimensional approach to the diffraction problem, except for the terms neglected so far.

The partial slit amplitudes $f_{\mathrm{l}}(\vartheta)$ and $f_{\mathrm{r}}(\vartheta)$ arising from the respective left and right half of each slit without corrugation can be extracted by the definitions

$$
f_{1}(\vartheta):=\int_{0}^{\frac{s}{2}} \mathrm{~d} \zeta \mathrm{e}^{\mathrm{i} \kappa\left(\frac{s}{2}-\zeta\right)} \mathrm{e}^{\mathrm{i} \varphi(\zeta)}
$$

and

$$
f_{\mathrm{r}}(\vartheta):=\int_{0}^{\frac{s}{2}} \mathrm{~d} \zeta \mathrm{e}^{-\mathrm{i} \kappa\left(\frac{s}{2}-\zeta\right)} \mathrm{e}^{\mathrm{i} \varphi(\zeta)}
$$

with $\kappa \equiv k \sin \vartheta$. It might be worth mentioning that the slit amplitude $f_{\text {slit }}(\vartheta)$ used in the non-corrugated case can be obtained by

$$
f_{\text {slit }}(\vartheta)=f_{1}(\vartheta)+f_{\mathrm{r}}(\vartheta)=2 \int_{0}^{\frac{s}{2}} \mathrm{~d} \zeta \cos \left[\kappa\left(\frac{s}{2}-\zeta\right)\right] \mathrm{e}^{\mathrm{i} \varphi(\zeta)} .
$$

This leads to a detection probability

$$
\begin{gathered}
I_{\Omega}(\vartheta) \propto \mid \sum_{j} \mathrm{e}^{-\mathrm{i} \kappa j d} \int_{-\frac{L_{y}}{2}}^{\frac{L_{y}}{2}} \mathrm{~d} y^{\prime}\left\{f_{\mathrm{r}}(\vartheta) \mathrm{e}^{-\mathrm{i} \kappa \Delta_{j}^{\mathrm{r}}\left(y^{\prime}\right)}+f_{1}(\vartheta) \mathrm{e}^{\mathrm{i} \kappa \Delta_{j}^{1}\left(y^{\prime}\right)}+\right. \\
\left.+\frac{1}{\mathrm{i} \kappa}\left[\mathrm{e}^{\mathrm{i} \kappa \Delta_{j}^{1}\left(y^{\prime}\right)}-\mathrm{e}^{-\mathrm{i} \kappa \Delta_{j}^{\mathrm{r}}\left(y^{\prime}\right)}\right]\right\}\left.\right|^{2} .
\end{gathered}
$$

For the principal maxima in the diffraction pattern one has $\vartheta=\vartheta_{n}$ and the momentum transfer $\kappa$ becomes $\kappa_{n}=\frac{2 \pi n}{d}$ so that

$$
\mathrm{e}^{-\mathrm{i} \kappa_{n} j d}=1 \text {. }
$$

After expanding the exponentials up to the second order the term in square brackets in Eq. (4.71) becomes for the $n$th principal maximum

$$
\begin{aligned}
\sum_{j} \int_{-\frac{L y}{2}}^{\frac{L y}{2}} \mathrm{~d} y^{\prime} \frac{1}{\mathrm{i} \kappa_{n}} & {\left[\mathrm{i} \kappa_{n} \Delta_{j}^{\mathrm{l}}\left(y^{\prime}\right)-\frac{\kappa_{n}^{2}}{2}\left(\Delta_{j}^{\mathrm{l}}\left(y^{\prime}\right)\right)^{2}+\mathrm{i} \kappa_{n} \Delta_{j}^{\mathrm{r}}\left(y^{\prime}\right)+\frac{\kappa_{n}^{2}}{2}\left(\Delta_{j}^{\mathrm{r}}\left(y^{\prime}\right)\right)^{2}\right] } \\
= & {\left[\left\langle\Delta_{j}^{\mathrm{l}}(y)\right\rangle_{j, y}+\frac{\mathrm{i} \kappa_{n}}{2}\left(\sigma_{j, y}^{\mathrm{l}}\right)^{2}+\left\langle\Delta_{j}^{\mathrm{r}}(y)\right\rangle_{j, y}-\frac{\mathrm{i} \kappa_{n}}{2}\left(\sigma_{j, y}^{\mathrm{r}}\right)^{2}\right], }
\end{aligned}
$$


where the expectation values \langle\rangle$_{j, y}$ and variances $\sigma_{j, y}^{2}$ refer to an average over the grating slits and the vertical coherence length $L_{y}$. Since the corrugation has been defined as the deviation from the mean value the expectation values are equal to zero. Furthermore there is no reason to believe the left and right bar edge corrugations have different statistics so one has

$$
\left(\sigma_{j, y}^{\mathrm{l}}\right)^{2}=\left(\sigma_{j, y}^{\mathrm{r}}\right)^{2}=:\left(\sigma_{j, y}\right)^{2}
$$

and, as a consequence, Eq. (4.73) is equal to zero. With this, Eq. (4.71) becomes

$$
I_{\Omega}\left(\vartheta_{n}\right) \propto f_{1}\left(\vartheta_{n}\right)\left\langle\mathrm{e}^{\mathrm{i} \kappa_{n} \Delta_{j}^{1}(y)}\right\rangle_{j, y}+f_{\mathrm{r}}\left(\vartheta_{n}\right)\left\langle\mathrm{e}^{-\mathrm{i} \kappa_{n} \Delta_{j}^{\mathrm{r}}(y)}\right\rangle_{j, y} .
$$

For typical experimental values the arguments of the exponentials are about $0.03 \times 2 \pi n$ which allows approximately to write for Eq. (4.75) the simple result

$$
I_{\Omega}\left(\vartheta_{n}\right) \propto \mathrm{e}^{-\kappa_{n}^{2} \sigma_{j, y}^{2}}\left|f_{\text {slit }}\left(\vartheta_{n}\right)\right|^{2} \equiv \mathrm{e}^{-\left(\frac{2 \pi n \sigma_{j, y}}{d}\right)^{2}}\left|f_{\text {slit }}\left(\vartheta_{n}\right)\right|^{2}
$$

The influence of grating imperfections has been studied by Grisenti et al. [9.9]. For the strictly two-dimensional case the authors derive a Debye-Waller-like damping factor

$$
I_{\Omega}\left(\vartheta_{n}\right) \propto \mathrm{e}^{-\left(\kappa_{n}^{2} \sigma_{0}\right)^{2}}\left|f_{\text {slit }}\left(\vartheta_{n}\right)\right|^{2}
$$

to the $n$th order intensity. The quantity $\sigma_{0}^{2}$ stands for the variance of the slit edge positions with respect to their mean values $j d \pm \frac{s}{2}$ as averaged over all illuminated slits $j$. This result is in agreement with Eq. (4.76) in the limit $L_{y} \rightarrow 0$, i.e. a vanishing coherence in $y$ direction of the incident beam. It is apparent from Eq. (4.76) that the inclusion of a finite coherence length leads also to a Debye-Waller damping, as long as the atom-bar interaction can be calculated with respect to a locally straight bar edge. For corrugations that vary strongly over small lengths $\Delta y$ each trajectory experiences a different potential than that assumed for the straight edge which leads to a dependence on $y$ of the partial slit amplitudes $f_{1}(\vartheta)$ and $f_{\mathrm{r}}(\vartheta)$ in Eq. (4.71) which can be expressed in terms of a $y$-dependent complex number $R(y)$ assigned to each of the amplitudes. If the corrugated surface potential is such that the regions where the potential is stronger due to the roughness find a complement in regions where it is weaker one may expect $\langle|R(y)|\rangle_{y}=1$ so that there is only a phase factor assigned to each amplitude which will also lead to a damping similar to that obtained above. For corrugated potentials that cannot be averaged to that of a locally straight edge (e.g. if the surface layer of the bar has a porous structure) the corrugation will yield a factor $R(y)$ whose modulus has an average different from zero. This means that in the latter case the diffraction intensities will be affected by the surface corrugation. A quantitative evaluation of these cases is numerically demanding and requires more detailed information on the bar surfaces than is available today. Recent progress in acquiring scanning electron analyses of the transmission gratings is expected to enable further work on this issue. 


\subsection{Retardation}

The effect of retardation can be included in the attractive dispersion potential $V_{3}(l)=-\frac{C_{3}}{l^{3}}$ for an atom at a distance $l$ from the plane surface of a dielectric by assigning an $l$-dependence to the dispersion interaction strength $C_{3}(l)$ which is given according to Eq. (3.25) in chapter 3 by

$$
C_{3}(l)=-\frac{\hbar}{4 \pi} \int_{0}^{\infty} \mathrm{d} \omega \alpha(\mathrm{i} \omega) g(\mathrm{i} \omega) \mathrm{e}^{-2 k l}\left(1+k l+k^{2} l^{2}\right),
$$

with $k \equiv \frac{\omega}{c}$, where $c$ stands for the speed of light. With the help of the procedures described in chapter 3 the polarizability $\alpha(\mathrm{i} \omega)$ of the atom and the electronic response $g(\mathrm{i} \omega)$ of the solid can be determined and the integral Eq. (4.78) can be evaluated numerically as is illustrated in Fig. 4.12 for helium atoms and a silicon nitride half-space. As explained earlier, $\alpha(\mathrm{i} \omega)$ has roughly the shape of the right half of a Lorentz curve centred at the origin. For ground-state helium, which has a high average excitation energy among all atoms considered in this work, the half-width of the curve of $\alpha(\mathrm{i} \omega)$ is large, so upon integration like in Eq. (4.78) a large portion of the area under the integrand function is taken away by the retardation factor $\mathrm{e}^{-2 k l}\left(1+k l+k^{2} l^{2}\right)$. In other words, the effect of retardation is more pronounced for helium than for most other atoms. Especially metastable atoms, whose average excitation energy is ten times smaller that that of groundstate atoms, are less affected by retardation. For example, $C_{3}(5 \mathrm{~nm})$ of metastable helium as shown in Fig. 4.12 decreases from the static value by only $5 \%$ whereas $C_{3}(5 \mathrm{~nm})$ of ground-state helium falls by $20 \%$.

The large drop of $C_{3}(l)$ of ground-state helium over distances of the order of the range of the dispersion interaction raises doubts if the dispersion interaction strength $C_{3}$ measured in diffraction experiments can really be identified with the non-retarded limit. This problem can be addressed by calculating a theoretical diffraction pattern with retardation and compare it with a corresponding nonretarded result. If the associated change in the detection probability $I_{\Omega}\left(\vartheta_{n}\right)$ for the $n$th order maximum in the diffraction patterns is small for ground-state helium atoms it can be concluded that the same is true for other atoms and especially for metastable species. In accordance with the theory set out in the previous sections the detection probability $I_{\Omega}\left(\vartheta_{n}\right)$ is practically determined by the phase

$$
\varphi(x):=-\frac{1}{\hbar v} \int_{-\infty}^{+\infty} \mathrm{d} z V^{\mathrm{att}}(x, z)
$$

whose definition Eq. (4.1) is restated here for clarity. Without any loss of significance the current investigation can be carried out assuming the atom-bar 


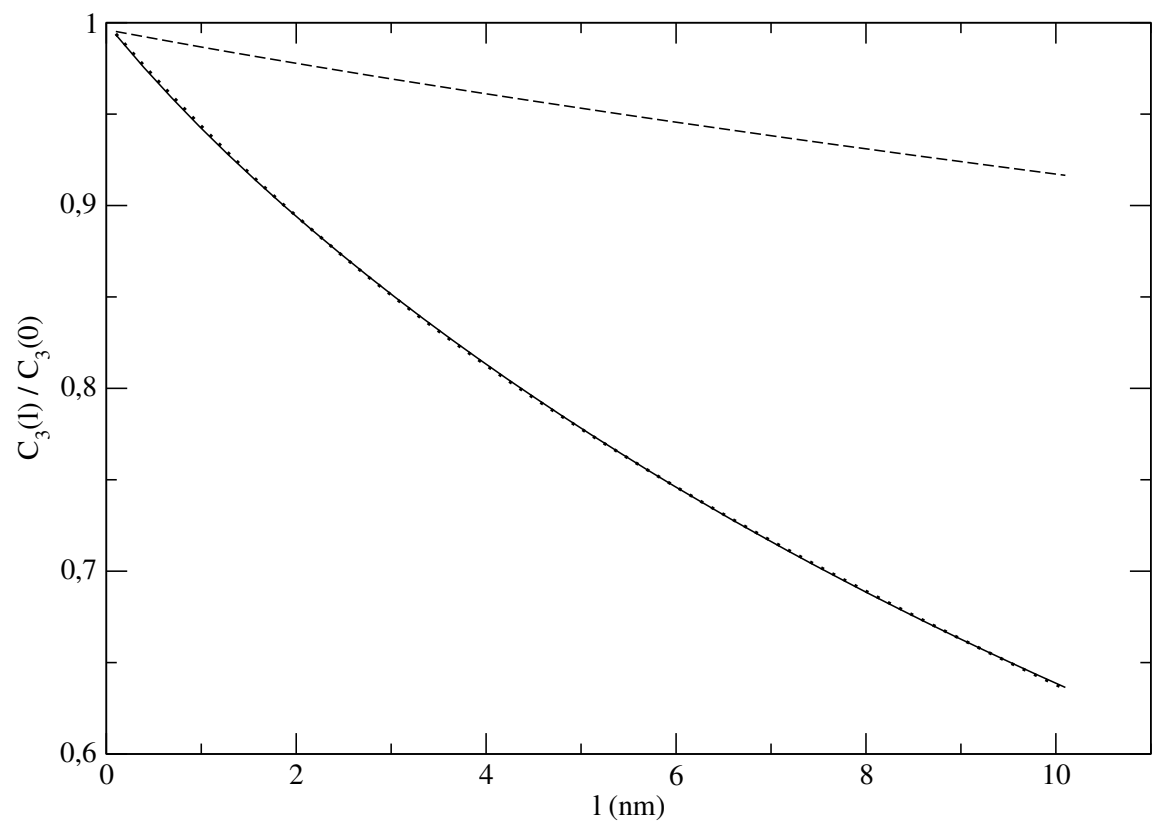

Figure 4.12: The dispersion interaction strength $\frac{C_{3}(l)}{C_{3}(0)}$ for ground-state helium atoms and a silicon nitride surface assumes a dependence on the distance $l$ if retardation is included (solid line). In order to later draw quantitative conclusions on the impact of the retardation the unnormalized curve has been fitted by the function $C_{3}(l)=$ $\left(C_{3}(0)+A_{0} l+A_{1} l^{2}\right) \mathrm{e}^{-A_{2} l}$ (dotted line), with $A_{0}=5.6898910^{-3}, A_{1}=4.9508910^{-4}$, and $A_{2}=0.102751$. For metastable helium atoms (dashed line) the effect of retardation is weaker because their average excitation energy is lower than for ground-state atoms.

interaction to be of the form

$$
V^{\mathrm{att}}(x, z)=V_{\mathrm{ret}}[l(x, z)]:=-\frac{C_{3}[l(x, z)]}{l^{3}(x, z)} .
$$

Hereby $l(x, z)$ stands for the perpendicular distance between the side of the grating bar facing the slit and the atomic trajectory. As Fig. 4.13 shows, this perpendicular distance is well-defined only for a section of the beam trajectory whose starting and end points are denoted by $z_{\mathrm{i}}$ and $z_{\mathrm{f}}$, respectively. As mentioned earlier in this text, the portions of the beam trajectory that are ignored by this procedure amount to a factor $\cos \beta$ in the resulting phase $\varphi(x)$, where $\beta$ is the wedge angle of the trapezoid-shaped bar cross-section. This technique to determine the phase $\varphi(x)$ that has been used in [12] for a non-retarded potential. It will become apparent in the following that an extension to retarded potentials is possible with the help of the fitting function

$$
C_{3}^{\mathrm{fit}}(l)=\left(C_{3}(0)+A_{0} l+A_{1} l^{2}\right) \mathrm{e}^{-A_{2} l}
$$

that paraphrases the exact expression Eq. (4.78). Since the fitting function has the same form as the retardation factor in the integrand of Eq. (4.78) it appears 


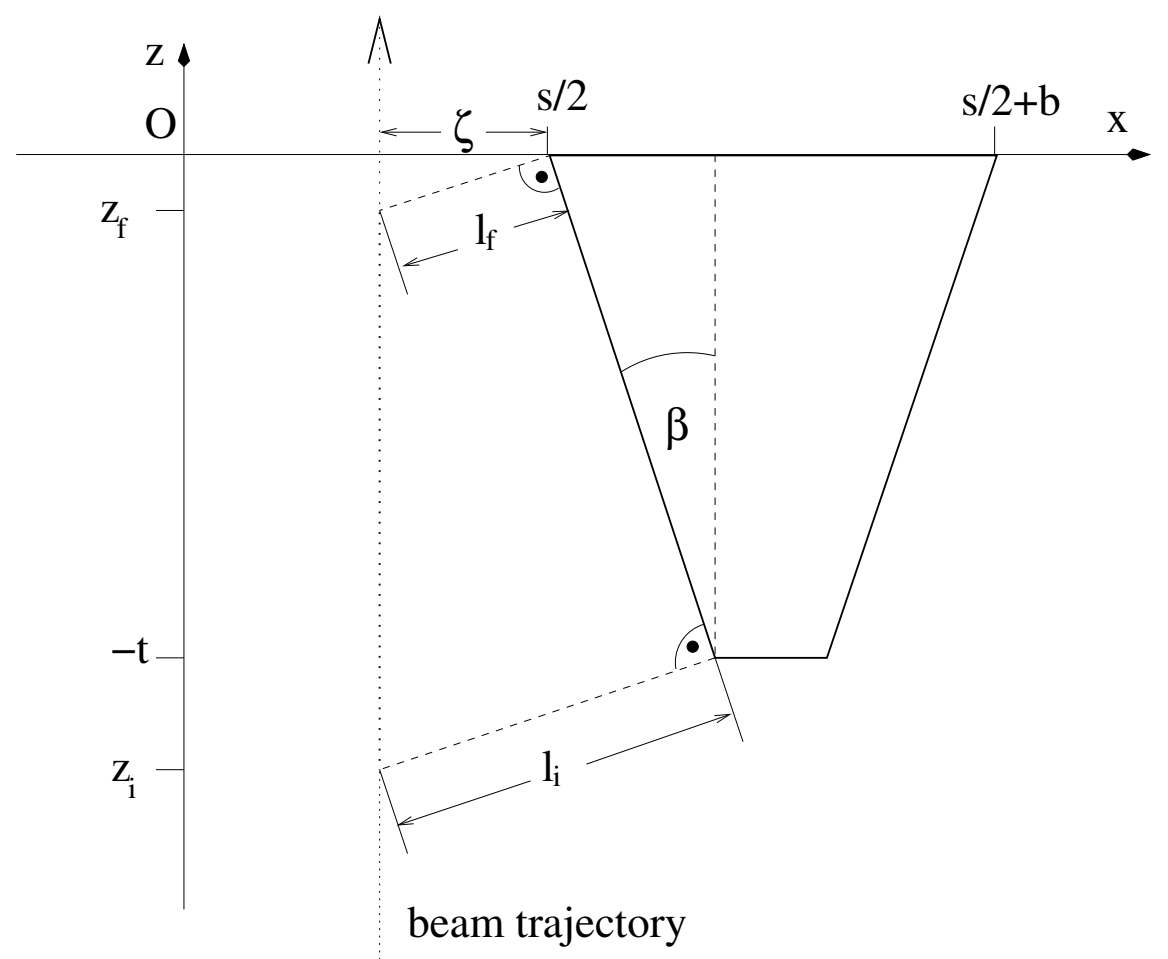

Figure 4.13: For the calculation of the quantum mechanical phase $\varphi(x)$ with the retarded bar potential the beam trajectories for an impact parameter $\zeta$ are integrated from $z_{i}$ to $z_{f}$, where the perpendicular distance $l$ to the bar surface is well-defined.

probable that there is an analytical solution to the integral Eq. (4.78) if the oneoscillator approximations Eq. (3.33) and Eq. (3.45), respectively, are used for the atomic polarizability $\alpha(\mathrm{i} \omega)$ and the response $g(\mathrm{i} \omega)$ of the solid. The main concern here is that for ground-state helium the approximation Eq. (4.81) works excellently for all distances $l$ at which the potential $V_{\text {ret }}[l(x, z)]$ significantly differs from zero. Fig. 4.12 illustrates the quality of the fit that is realized with the parameter set $A_{0}=5.6898910^{-3}, A_{1}=4.9508910^{-4}$, and $A_{2}=0.102751$.

With these considerations, the phase $\varphi(x)$ becomes for ground-state helium atoms

$$
\varphi(x)=-\frac{1}{\hbar v} \int_{z_{\mathrm{i}}}^{z_{\mathrm{f}}} \mathrm{d} z\left(\frac{C_{3}(0)}{l(x, z)^{3}}+\frac{A_{0}}{l(x, z)^{2}}+\frac{A_{1}}{l(x, z)}\right) \mathrm{e}^{-A_{2} l(x, z)}
$$

From the figure Fig. 4.13 it can be derived by elementary geometrical methods that

$$
l\left(x, z_{\mathrm{i}}\right) \equiv l_{\mathrm{i}}(x)=\frac{\zeta+t \tan \beta}{\cos \beta}
$$


and

$$
l\left(x, z_{\mathrm{f}}\right) \equiv l_{\mathrm{f}}(x)=\frac{\zeta}{\cos \beta},
$$

where the usual impact parameter $\zeta:=\frac{s}{2}-x$ has been introduced. One also finds

$$
l(x, z)=\zeta \cos \beta-z \sin \beta,
$$

which can be used to substitute in Eq. (4.82). This yields

$$
\varphi(x)=\frac{1}{\hbar v \sin \beta} \int_{l_{\mathrm{f}}(x)}^{l_{\mathrm{i}}(x)} \mathrm{d} l\left(\frac{C_{3}(0)}{l^{3}}+\frac{A_{0}}{l^{2}}+\frac{A_{1}}{l}\right) \mathrm{e}^{-A_{2} l} .
$$

The evaluation of this integral is of mainly technical interest as may be checked in the appendix. As a result, one obtains

$$
\begin{aligned}
\varphi(x) & =\frac{1}{2 \hbar v \tan \beta}\left\{\mathrm{e}^{-\frac{d \zeta}{\cos \beta}}\left[\frac{C_{3}(0) \cos \beta}{\zeta^{2}}-\frac{K_{2}}{\zeta}\right]-\right. \\
& \left.-\mathrm{e}^{-\frac{d(\zeta+t \tan \beta)}{\cos \beta}}\left[\frac{C_{3}(0) \cos \beta}{(\zeta+t \tan \beta)^{2}}-\frac{K_{2}}{\zeta+t \tan \beta}\right]\right\}+ \\
& +\frac{K_{1}}{\hbar v \sin \beta}\left\{\ln \left(1+\frac{t \tan \beta}{\zeta}\right)-\sum_{n=1}^{\infty}\left(\frac{-A_{2}}{\cos \beta}\right)^{n} \frac{(\zeta+t \tan \beta)^{n}-\zeta^{n}}{n n !}\right\},
\end{aligned}
$$

with

$$
K_{1}:=A_{1}-A_{2} A_{0}+\frac{C_{3}(0) A_{2}^{2}}{2}
$$

and

$$
K_{2}:=C_{3}(0) A_{2}-2 A_{0} .
$$

The non-retarded limit of the lengthy expression Eq. (4.87) is obtained by setting all parameters $A_{i}$ equal to zero so that both $K_{i}$ also vanish. This yields

$$
\varphi(x)=\frac{C_{3}(0) \cos \beta}{2 \hbar v \tan \beta}\left\{\frac{1}{\zeta^{2}}-\frac{1}{(\zeta+t \tan \beta)^{2}}\right\},
$$

which is equivalent to the result stated in Ref. [12].

The phase $\varphi(x)$ according to Eq. (4.87) is compared with the phase for the non-retarded case in Fig. 4.14. It can be seen that despite the large variation of $C_{3}(l)$ (cf. Fig. 4.12) the phase $\varphi(x)$ changes only by a little because the side of the bar is not parallel to the beam trajectory but inclined by the wedge angle $\beta$ of the bar. The figure shows that for a bar depth $t=57 \mathrm{~nm}$ and $\beta=11^{\circ}$ the difference between the retarded and the non-retarded phase is smaller than 


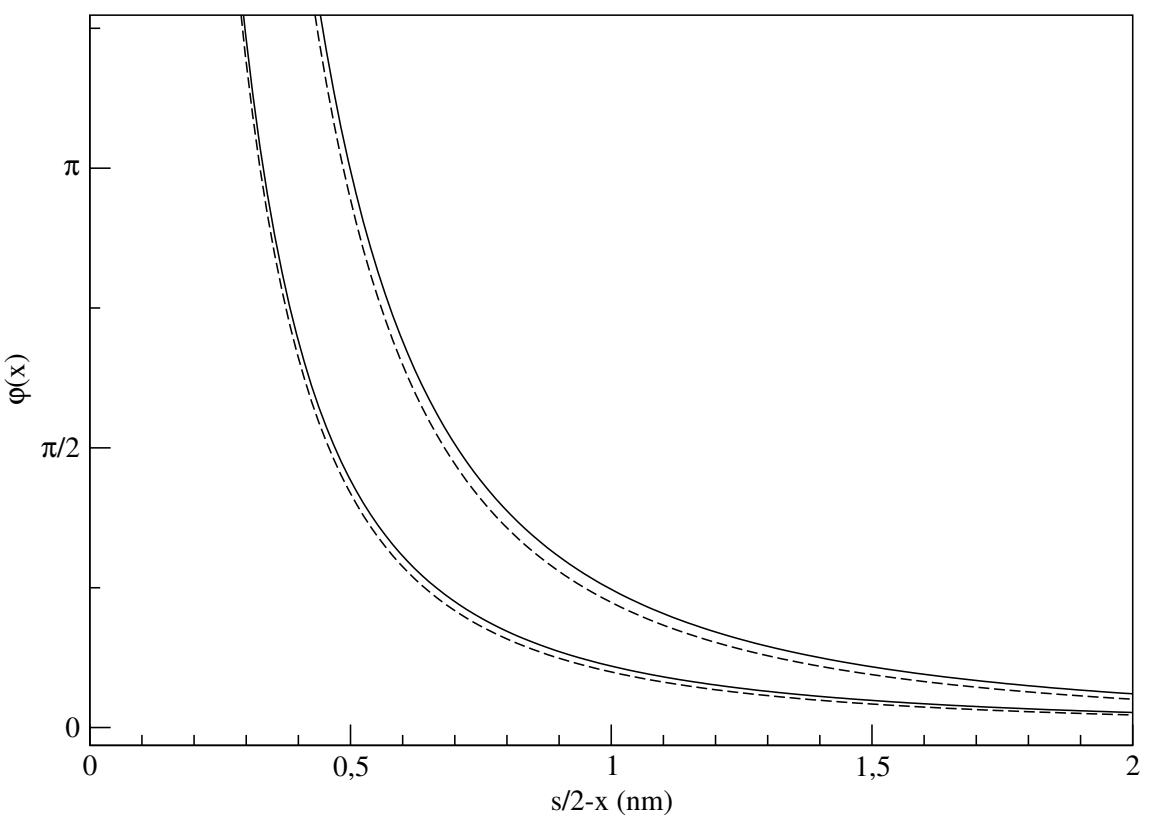

Figure 4.14: The inclusion of retardation in the atom-bar potential leads to a small reduction of the quantum mechanical phase $\varphi(x)$ which determines the diffracted intensities. The lower solid line accounts for the non-retarded case of ground-state helium atoms with a grating whose bars have a wedge angle of $\beta=11^{\circ}$ and a depth $t=57 \mathrm{~nm}$. The corresponding curve with the retardation included (lower dashed line) is only slightly smaller. For the new generation of gratings with $\beta=5^{\circ}$ and $t=100 \mathrm{~nm}$ the difference between the non-retarded (upper solid line) and the retarded case (upper dashed line) is more pronounced. All curves increase as they approach the bar edge at $\zeta \equiv \frac{s}{2}-x=0$ because there the atom-bar interaction is strongest.

for a bar with $t=100 \mathrm{~nm}$ and $\beta=5^{\circ}$ which is roughly the geometry of the new generation of transmission gratings used in current experiments. The change of the phase $\varphi(x)$ due to retardation leads to a change in the principal diffraction intensities $I_{\Omega}\left(\vartheta_{n}\right)$ that is summarized in table 4.1 for both bar geometries. As it turns out, the small 3rd, 6th and 9th order intensities of the non-retarded case are reduced by about $10 \%$ as a consequence of retardation while the higher intensities of the other orders are affected by about $1 \%$. For the bar with the smaller wedge angle the effect is slightly more pronounced as can be expected from the behaviour of the phase shown in Fig.4.14. In sum, the influence of retardation on the diffraction intensities is a few-percent-effect for ground-state helium atoms. As these atoms are used to determine the slit width $s$ of the grating that has been employed for the diffraction of metastable atoms it will be checked in chapter 5 if the resulting $s$ has to be interpreted in terms of the retardation. For the diffraction of metastable atoms itself the effect of retardation can be expected to be significantly smaller than for ground-state helium atoms so 


\begin{tabular}{c||c|c|c||c}
$n$ & $I_{\Omega}\left(\vartheta_{n}\right) / I_{\Omega}(0)$ & $I_{\Omega}^{\mathrm{ret}}\left(\vartheta_{n}\right) / I_{\Omega}^{\mathrm{ret}}(0)$ & $\gamma(\%)$ & $\gamma(\%)$ (new grating) \\
\hline \hline 1 & 0.1868 & 0.1861 & -0.4 & -0.7 \\
2 & 0.04011 & 0.04222 & 0.3 & 0.5 \\
3 & 0.0008286 & 0.0007315 & -13.3 & -12.8 \\
4 & 0.01309 & 0.01300 & -0.7 & -1.1 \\
5 & 0.005438 & 0.005479 & 0.8 & 1.0 \\
6 & 0.0007189 & 0.0006512 & -10.4 & -10.0 \\
7 & 0.004608 & 0.004578 & -0.7 & -0.9 \\
8 & 0.001814 & 0.001829 & 0.8 & 0.0 \\
9 & 0.0006241 & 0.0005755 & -8.4 & -8.0 \\
10 & 0.002360 & 0.002349 & -0.5 & -0.6
\end{tabular}

Table 4.1: Comparison of relative theoretical diffraction intensities of ground-state helium with (second column) and without (third column) retardation. The calculation is based on grating bars with a wedge angle $\beta=11^{\circ}$ and a depth $t=54 \mathrm{~nm}$. In the fourth and fifth columns the relative deviations $\gamma$ of the $n$th order intensities with and without retardation are compared, respectively, for this grating bar geometry which corresponds to the gratings used for metastable atoms, and for the new generation of gratings with $\beta=5^{\circ}$ and $t=100 \mathrm{~nm}$ which are used in current experiments.

that it can be neglected. The distinct deviation of some non-retarded diffraction intensities from those of the retarded case, especially for the new generation of gratings with small wedge angles, opens the challenging perspective of measuring for the first time atom-surface potentials in an intermediate range of distances where the retardation effects set in.

\subsection{Adsorbate}

The method of measuring atom-surface interaction potentials with the help of atom diffraction from transmission gratings is a tool which obviously becomes more valuable if it can be applied to a wider variety of atoms and surfaces. While it poses no principal problems to add beam sources of a great number of different atoms and molecules to the diffraction apparatus the surface they interact with has to stay the same as, in fact, there are no transmission gratings of the desired quality made from other materials than silicon nitride 1 . A way to circumvent this shortcoming is to cover the grating bars with a layer of the material whose

\footnotetext{
${ }^{1}$ Gold gratings may be an exception, although by judging from scanning electron micrographs and diffraction experiments silicon nitride gratings seem to have a significantly better quality.
} 
interaction with the atomic beam one wants to study. In view of modern deposit techniques this approach is much more feasible than trying to make good gratings from materials that are less rigid than the ceramic-like silicon nitride compounds.

With the theory presented so far the diffraction of atoms from a transmission grating, whose bar surfaces are covered with an adsorbate layer, can be easily described. The simplest model that reveals all the essential details is illustrated schematically in Fig.4.15. Within the model, all grating bar sides are covered

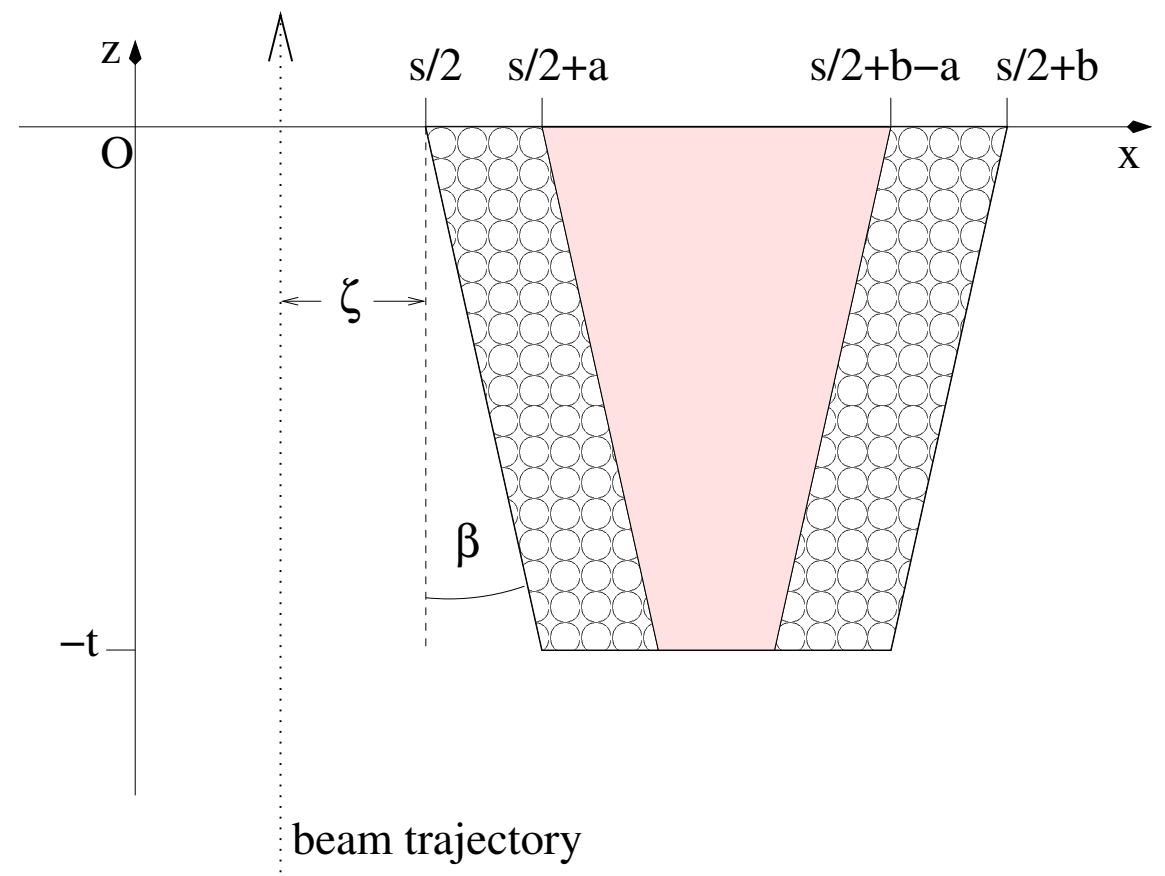

Figure 4.15: The figure shows a model of a grating bar of width $b-2 a$ which is covered with adsorbate layers of thickness $a$ on both sides. The model is used to calculate the quantum mechanical phase $\varphi(x)$ which determines the diffraction intensities.

with a layer of thickness $a$ that consists of a material whose interaction with the atomic beam is characterised by the strength coefficient $\tilde{C}_{3}$. The shape of the layers is chosen such that the bar cross-section with the layers added is an ideal trapezoid. As the grating is not rotated with respect to the incident beam the atom-bar potential is practically equal to zero in the middle region of the slit. Then the detection probability

$$
I_{\Omega}\left(\vartheta_{n}\right) \propto\left|f_{\text {slit }}\left(\vartheta_{n}\right)\right|^{2},
$$

associated with the $n$th order maximum in the diffraction pattern, depends on the adsorbate situation by the quantum mechanical phase $\varphi(x)$ that appears in 


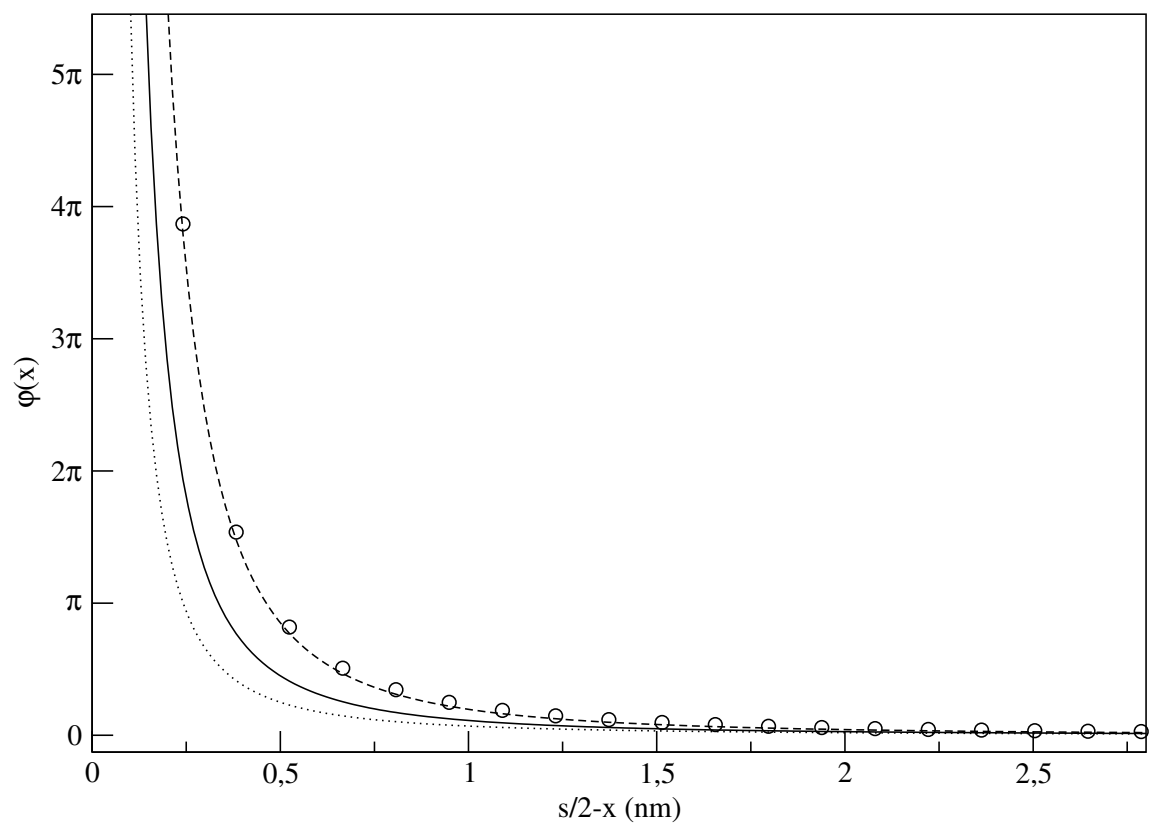

Figure 4.16: The quantum mechanical phase $\varphi(x)$ of ground-state helium atoms is plotted for grating bars that are covered with $1 \mathrm{~nm}$-thick adsorbate layers whose dispersion interaction strength $\tilde{C}_{3}$ is half (dotted line), equal (solid line), or twice (dashed line) $C_{3}$ of the core silicon nitride material of the bar. The circles are the result of a calculation for a bar that consists entirely of a material with $\tilde{C}_{3}=2 C_{3}$. As the circles nearly fall on the dashed curve it can be concluded that the phase $\varphi(x)$ is mostly determined by the dispersion interaction strength $\tilde{C}_{3}$ of the $1 \mathrm{~nm}$-thick surface layer.

the slit amplitude

$$
f_{\text {slit }}\left(\vartheta_{n}\right)=2 \int_{0}^{\frac{s}{2}} \mathrm{~d} \zeta \cos \left[\kappa_{n}\left(\frac{s}{2}-\zeta\right)\right] \mathrm{e}^{\mathrm{i} \varphi(x)},
$$

where the impact parameter $\zeta:=\frac{s}{2}-x$ with respect to the layer surface has been defined. The phase

$$
\varphi(x)=: \varphi_{1}(x)+\varphi_{2}(x)-\varphi_{3}(x)
$$

is written as a sum of three terms each of which can be calculated like in Eq. (4.14), while the terms containing the bar width $b$ and those arising from the atom-bar repulsion can be dropped in the present investigation. In Eq. (4.93), the first term

$$
\varphi_{1}(x)=\frac{t C_{3}}{\hbar v(\zeta+a)^{3}} \frac{1+\frac{t}{2(\zeta+a)} \tan \beta}{\left(1+\frac{t}{\zeta+a} \tan \beta\right)^{2}}
$$

accounts for the interaction of the beam trajectory with the silicon nitride core of the bar, whose base line width is $b-2 a$. Due to the adsorbate layer the beam 
trajectory cannot get closer to the core surface than $a$. The second term

$$
\varphi_{2}(x)=\frac{t \tilde{C}_{3}}{\hbar v \zeta^{3}} \frac{1+\frac{t}{2 \zeta} \tan \beta}{\left(1+\frac{t}{\zeta} \tan \beta\right)^{2}}
$$

describes the interaction with a bar of width $b$ which consists entirely of the adsorbate material. In reality, only the layers on the surface consist of this material, therefore an appropriately sized core of the same material is subtracted in order to get the correct result. In Eq. (4.93), this is accomplished by the term

$$
\varphi_{3}(x)=\frac{t \tilde{C}_{3}}{\hbar v(\zeta+a)^{3}} \frac{1+\frac{t}{2(\zeta+a)} \tan \beta}{\left(1+\frac{t}{\zeta+a} \tan \beta\right)^{2}} .
$$

In total, one obtains

$$
\varphi(x)=\frac{t}{\hbar v}\left\{\frac{\tilde{C}_{3}}{\zeta^{3}} \frac{1+\frac{t}{2 \zeta} \tan \beta}{\left(1+\frac{t}{\zeta} \tan \beta\right)^{2}}-\frac{\left(\tilde{C}_{3}-C_{3}\right)}{(\zeta+a)^{3}} \frac{1+\frac{t}{2(\zeta+a)} \tan \beta}{\left(1+\frac{t}{\zeta+a} \tan \beta\right)^{2}}\right\} .
$$

Fig. 4.16 shows plots of the phase $\varphi(x)$ in the vicinity of the bar surface covered with a $1 \mathrm{~nm}$ thick adsorbate layer for the cases $\tilde{C}_{3}=2 C_{3}$ and $\tilde{C}_{3}=0.5 C_{3}$. As one intuitively expects, the respective curves run above and below that with $\tilde{C}_{3}=C_{3}$, i.e. the adsorbate layers consists of the same material as the core of the bar. The case $\tilde{C}_{3}=2 C_{3}$ is also compared with the phase arising from a bar that consists entirely of the adsorbate material. As the two curves are nearly identical it can be concluded that the phase effectively depends only on a 1 or $2 \mathrm{~nm}$-thick skin of the bar whereas the bulk material is of minor importance. In view of an application of this effect as described in the introduction to this section it is important to see if the distinct character of the phases persists in the actual diffraction intensities. That this is indeed true is illustrated in Fig. 4.17 where a comparison of the principal diffraction intensities is made for the same cases as set out above. The fact that the diffraction intensities for a bar covered with a $1 \mathrm{~nm}$ thick layer of material A are very close to those of a homogenous bar made of A speaks much in favour of an extended use of the on-going diffraction experiments whereby the grating bars are to be covered with certain substances in order to measure their interaction potential with the atoms in the beam.

\subsection{Summary}

This chapter has demonstrated how the fundamental results of the previous chapters can be applied to the diffraction of atoms from transmission gratings of a certain shape and material. Through detailed investigations the versatility of the experimental scheme has been improved in a number of ways. 


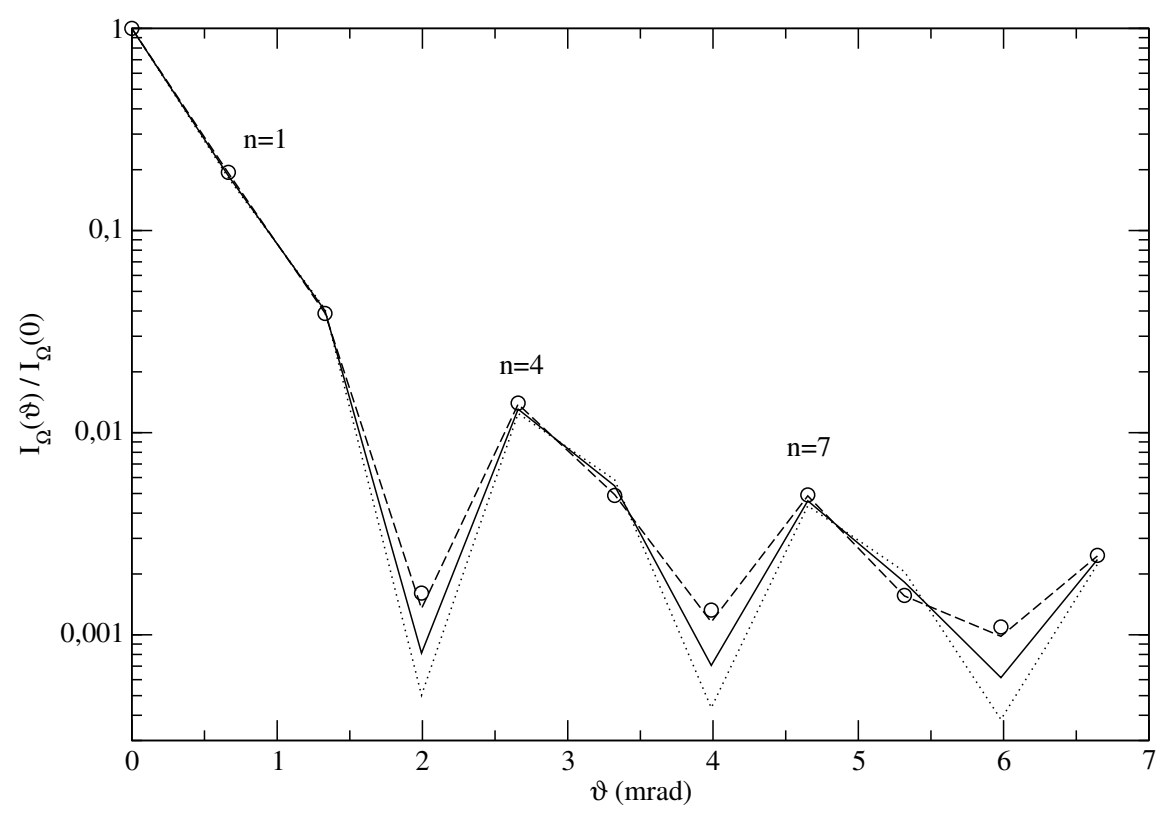

Figure 4.17: The theoretical relative principal order diffraction intensities are plotted versus the diffraction angle for gratings whose bar sides are covered with a $1 \mathrm{~nm}$-thick layer of adsorbate material whose dispersion interaction strength $\tilde{C}_{3}$ with the diffracted ground-state helium atoms is half (dotted line), equal (solid line), or twice (dashed line) $C_{3}$ of the silicon nitride core material of the bar. The circles are calculated for bars that consist entirely of a material with $\tilde{C}_{3}=2 C_{3}$. As the circles practically reproduce the intensities calculated for only a top layer with $\tilde{C}_{3}=2 C_{3}$ it is evident that for groundstate helium the diffraction intensities are determined by the dispersion interaction strength of the adsorbate layer.

- The theoretical description of atom diffraction with rotated gratings is used in chapter 5 to determine the grating geometry and is of central relevance for future experiments.

- For the first time the influence of retardation is described in quantitative terms which opens the way for future pioneering projects on this subject.

- By theoretically accounting for adsorbate layers on the transmission gratings it is made possible to extend the present measurements of dispersion interaction constants to many materials and thus create an experimental standard which will be of great use for all further work on atom-surface potentials.

- The detailed account of the grating bar corrugation, together with the above items helps in chapter 5 to find an explanation to unresolved deviations of the measured diffraction intensities from theory and completes the theoreti- 
cal description of the given type of diffraction experiments with transmission gratings. 


\section{Chapter 5}

\section{Diffraction of Metastable Atoms}

The purpose of this chapter is to determine the dispersion interaction constants $C_{3}\left(\mathrm{He}^{*}\right)$ and $C_{3}\left(\mathrm{Ne}^{*}\right)$ of metastable helium and neon atoms from measured diffraction patterns obtained with a silicon nitride transmission grating of the type frequently discussed in the previous chapter. As the metastable species decay quickly to their respective ground-states when in direct contact with a surface [104, 105, 106, 107] the dispersion interaction strength cannot easily be measured from surface scattering experiments. The atom-optical method is unique in that the metastable atoms remain intact while scanning the grating bar potential. After the possibility of diffracting metastable rare-gas atoms from nanostructured transmission gratings has been demonstrated by O. Carnal et al. [6] the study presented in the following is of great value for recently started experiments of a French group concerned with inelastic scattering processes which metastable atoms undergo upon passage through a transmission grating [14]. The present method marks a progress from earlier attempts at measuring the dispersion interaction strength of fragile atoms being in metastable or Rydberg states [108, 10.9, 110, 52, 117, 112, 9.3, 16, 15]] because not least due to the high quality of the used transmission gratings very accurate results can be achieved. Furthermore, the small period $(100 \mathrm{~nm})$ of the gratings offers a large surface to interact with the incident beam so that the signal of atoms actually in contact with the surface potential is higher than for single cavities or slits. Finally, the small grating period guarantees only the non-retarded potential to be scanned which is not possible with wide slit or cavities.

Parts of the following investigation, in particular those concerned with the geometry of the grating, are based on results obtained by the diffraction of groundstate helium atoms. In order to achieve significant results for the dispersion interaction strength $C_{3}$ of metastable atoms it is necessary to review in some detail the experimental and theoretical concepts of the ground-state helium diffraction, and to scrutinize the actual experiment carried out with the metastable species. It will become apparent in the course of this chapter that the careful analysis 
which is based on the theoretical studies of the previous chapter does in fact help to clarify and refine the experimental and theoretical approaches so that new interesting aspects become visible at which future experiments can be aimed.

The diffraction apparatus used for the experiments with metastable atoms is shown as a schematic drawing in Fig. 5.1. It reflects the data given in [54]. The

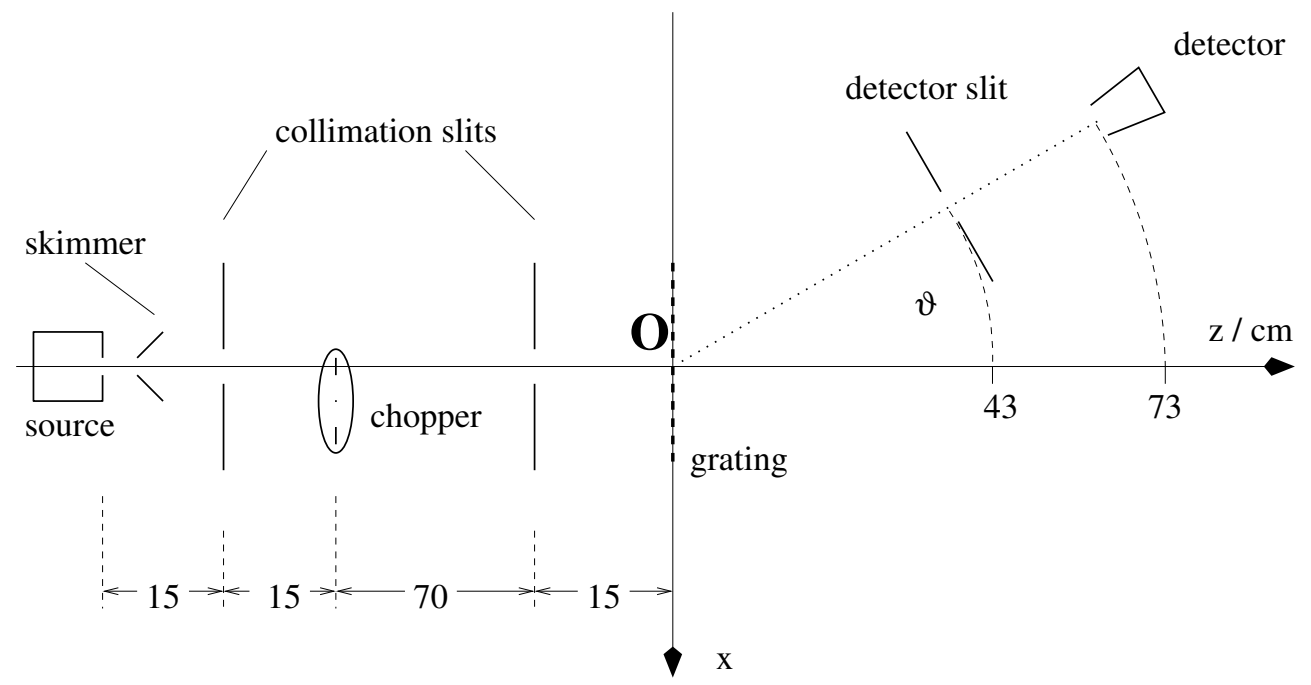

Figure 5.1: Experimental set-up for the diffraction of metastable atoms. A rotating slotted disk (chopper) between the first and the second collimator periodically blocks and transmits the atomic beam. When one of the slots passes through the beam the detector is triggered to await the arrival of a flock of atoms. Upon arrival at the detector the atoms are counted in different channels according to their time-of-flight (TOF). By this means many diffraction patterns of atoms can be recorded simultaneously whose individual velocity range is five times narrower than that of the source without the TOF device and whose resolution is considerably enhanced.

beam of metastable atoms is emitted from a source whose details are explained in section 5.4.1 and is pre-shaped by the first collimator. The bulk of the beam is then periodically blocked and transmitted by a rotating slotted disk (chopper) such that the intervals between subsequently transmitted flocks of atoms are long enough to avoid a simultaneous arrival at the detector of the slowest atoms of the first group with the fastest ones of the following group. By means of an appropriate triggering device the atoms arriving at the detector are counted into several channels according to the time that has passed since one of the slots in the chopper disk allowed the beam to pass. With this time-of-flight (TOF) selection of the atoms several diffraction patterns for different particle velocities are recorded at one angular scan of the detector. The procedure is necessary as the source for metastable atoms produces a beam with a wide velocity distribution which leads to high-order principal maxima in the diffraction patterns that are wider than 
the spacing in between, so, in effect, the high-order maxima overlap unless the TOF device is used which pre-selects atoms within a narrow range of velocities. The remaining aspects of the diffraction apparatus are explained in the following sections.

\subsection{The Transmission Grating}

The gratings used in the diffraction experiments have been manufactured by T. Savas and co-workers at the MIT. They are delivered on silicon chips whose size is about $1 \mathrm{~cm} \times 1 \mathrm{~cm}$ and whose thickness is $400 \mu \mathrm{m}$. In each chip there are three parallel rectangular windows that are barred by the actual transmission gratings levelling with the bottom plane of the chip. The grooves in the chip surface that form the windows are $5 \mathrm{~mm}$ long and trough-shaped (a trapezoidal prism with its narrow face in the bottom plane of the chip and a wedge angle of about $35^{\circ}$ ) the top side width being $2 \mathrm{~mm}$ and the bottom side $1.44 \mathrm{~mm}$, as specified for the particular chip number 5-3. The right window on this chip harbours the grating 5-3-1 that was used for the diffraction of metastable atoms. Fig. 5.2 illustrates the geometry described so far.
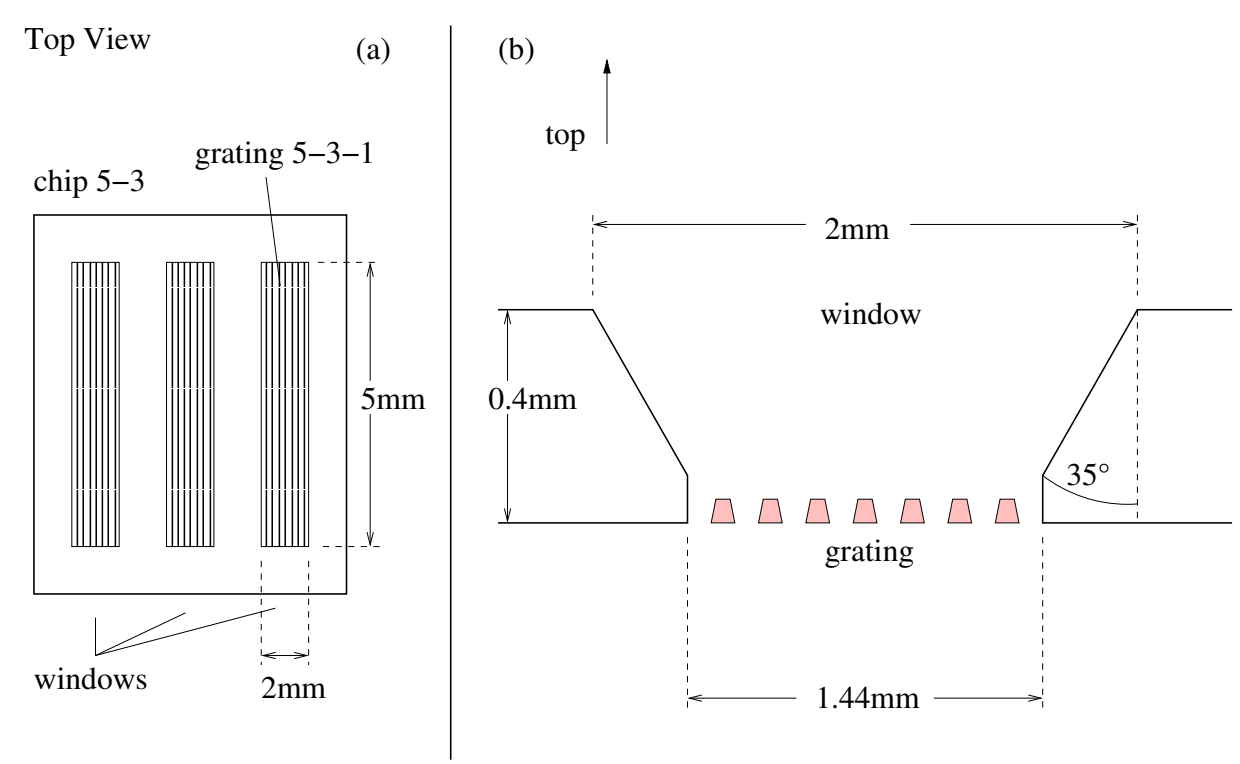

Figure 5.2: The transmission grating 5-3-1 used for the diffraction of metastable atoms is delivered on a silicon chip as shown on the left panel (a). For experiments with a rotated grating it is important to know that the actual grating bars are situated at the bottom of a window through the chip (b). 


\subsubsection{Period}

The period $d$ of the grating 5-3-1 as calibrated by the manufacturers is $d=$ $100 \pm 0.01 \mathrm{~nm}$ [98]. For the interpretation of the measured diffraction patterns of metastable atoms in terms of their dispersion interaction strength $C_{3}$ it is important to know the period $d$ of the grating to a better accuracy than $1 \%$. This is because the method that helps to determine the slit width $s$ of the grating is only sensitive to the ratio $\frac{s}{d}$. By standard error propagation laws a $1 \%$ deviation of the period $d$ would likewise affect the value of the slit width $s$, but for a reasonably accurate determination of the dispersion coefficients $C_{3}$ it has proven necessary to have the value of the slit width $s$ given within less than $1 \%$ error. In view of the delicate scales involved, is not considered acceptable to just believe the calibrated value of the period $d$ that is delivered along with the very gratings, instead, several sets of measured data are interpreted in terms of $d$ and are compared with the given value.

In order to gain information on the period $d$ it is sufficient to consider the diffraction of atoms by a transmission grating in the Fraunhofer limit. The principal maxima of order $n$ in the measured diffraction pattern are expected to be found at angles $\vartheta_{n}=\arcsin \left(\frac{n \lambda}{d}\right) \approx \frac{n \lambda}{d}$ for experimentally relevant values of $n \leq 15$ and of the atomic de Broglie wavelength $\lambda \approx 0.01 \mathrm{~nm}$. Extracting the principal diffraction angles $\vartheta_{n}$ from the measured diffraction pattern for a given time-of-flight $\tau$ it is possible to determine the period $d$ of the grating by

$$
d=\frac{n \lambda}{\vartheta_{n}}=\frac{n 2 \pi \hbar}{m v \vartheta_{n}}=\frac{n 2 \pi \hbar \tau}{m L \vartheta_{n}}
$$

where $L$ is the distance from the chopper to the detector (cf. Fig. 5.1) and $m$ stands for the mass of the atoms. An analysis of 10 diffraction patterns of metastable $\mathrm{He}^{*}$ with $\tau$ evenly distributed over a range of $0.665 \mathrm{~ms}<\tau<0.959 \mathrm{~ms}$ yields $L d=(193940 \pm 496) 10^{-9} \mathrm{~m}^{2}$. From the experimental set-up as shown in Fig. 5.1 one has $L=(158 \pm 1) \mathrm{cm}$, which leads to $d=(122.75 \pm 1.09) \mathrm{nm}$. An analogous procedure applied to six diffraction patterns of metastable $\mathrm{Ne}^{*}$ with $1.75 \mathrm{~ms}<\tau<2.3 \mathrm{~ms}$ yields $d=(98.9 \pm 2.4) \mathrm{nm}$. After a check of the record in the laboratory books it appears highly probable that the result obtained with $\mathrm{He}^{*}$ is systematically wrong due to a scaling factor that has been erroneously forgotten to be applied to the flight times $\tau$. Leaving $\mathrm{He}^{*}$ aside it remains to say that the result for the grating period $d$ as obtained from the $\mathrm{Ne}^{*}$ data has too large errors which is due to the fact that the peak positions in the measured $\mathrm{Ne}^{*}$ diffraction patterns are not equally spaced, as opposed to theoretical predictions. This discrepancy is due to systematical errors of the apparatus that are explained further in section 5.4 .3 of this work.

These errors have been recognized and subsequently eliminated by R. Brühl who has then recorded a series of diffraction patterns for ground-state He atoms 
at various velocities. The diffraction of metastable atoms cannot be repeated because the beam source has been removed since the first experiment and is currently being re-designed for future projects. For ground-state He atoms it lies in the nature of the beam source that the atomic beam has a sharp velocity distribution so that no TOF device is needed in order to obtain clearly separate principal peaks in the measured diffraction patterns. Despite the lack of a flight time $\tau$ in Eq. (5.1) the formula can be used. Treating the He gas in the source as an ideal gas one obtains from elementary thermodynamics for the mean velocity of the atomic beam

$$
v=\sqrt{\frac{5 k_{\mathrm{B}} T}{m}}
$$

where $k_{\mathrm{B}}$ is Boltzmann's constant and $T$ is the temperature of the beam source, which can (non-trivially) be assumed in our case to be equal to that of the He gas inside. Using the principal diffraction angles $\vartheta_{n}$ of 9 measured ground-state He diffraction patterns at 8 different source temperatures $12 \mathrm{~K}<T<350 \mathrm{~K}$, and inserting the beam velocity $v(T) \equiv \frac{L}{\tau}$ according to Eq. (5.2) one obtains from Eq. (5.1) for the grating period $d=(100.02 \pm 1.05) \mathrm{nm}$, which is in good agreement with the value $d=100 \mathrm{~nm}$ as given by the manufacturers of the grating.

To round the picture off and taking the opportunity of an atomic force microscope (AFM) being available, the bottom plane of the chip 5-3 which contains the relevant grating 5-3-1 has been scanned with that device. Scaling errors have been avoided by measuring the distance between the edges of two grating bars 20 periods apart from each other and by measuring immediately afterwards the distance between two marks on a commercially available calibration grid for AFM tools. The result $d=(104.3 \pm 1.0) \mathrm{nm}$ which has not been altered significantly with two other gratings of the same nominal slit width being scanned is now supposed to arise from the calibration grid to be faulty in terms of the stated errors. In an attempt to have the calibration grid compared at the MIT to the scale of the manufacturers, the tool disappeared in the mail which is likely to be due to very strict controls during the weeks after September 11th 2001. On the other hand, the manufacturers communicated that the very transmission gratings are actually being sold in the US as calibration tools and that in view of this they have been subject to a check at the National Institute of Standards and Technology (NIST) who have found no fault with them. All of this may be seen as a motivation to assume the period of the grating 5-3-1 to be $d=100 \mathrm{~nm}$ in the following.

\subsubsection{Shape of the Grating Bars}

From the scanning electron micrograph image Fig. 4.2 of a transmission grating similar to the one (5-3-1) used in the actual experiment with metastable atoms it 
can be inferred that the grating bars have a shape close to a symmetrical trapezoidal prism. In order to determine the ratio $\frac{t}{s}$ of the depth $t$ of the grating bars and the slit width $s$ and also the wedge angle $\beta$ of the trapezoid, the transmitted intensity of a beam of ground-state He atoms is measured while the grating is rotated around the vertical axis that passes through the centre of the illuminated spot on the grating. There have been some inaccuracies in earlier experiments with this purpose. In particular, they have led to values of the depth $t$ and the wedge angle $\beta$ of the bars of the relevant grating 5-3-1 which cannot be matched to form a trapezoid, or even a triangle. Motivated by this the experimental as well as the theoretical approach have been refined. On the experimental side, R. Brühl has made sure the rotation axis be really at the beam centre and also that the incident beam hit a region on the chip in the middle of the window that frames the grating under investigation. By this it can be avoided that parts of the incident beam are blocked by the frame which otherwise could wander into the beam path upon rotation of the grating (see Fig. 5.3).

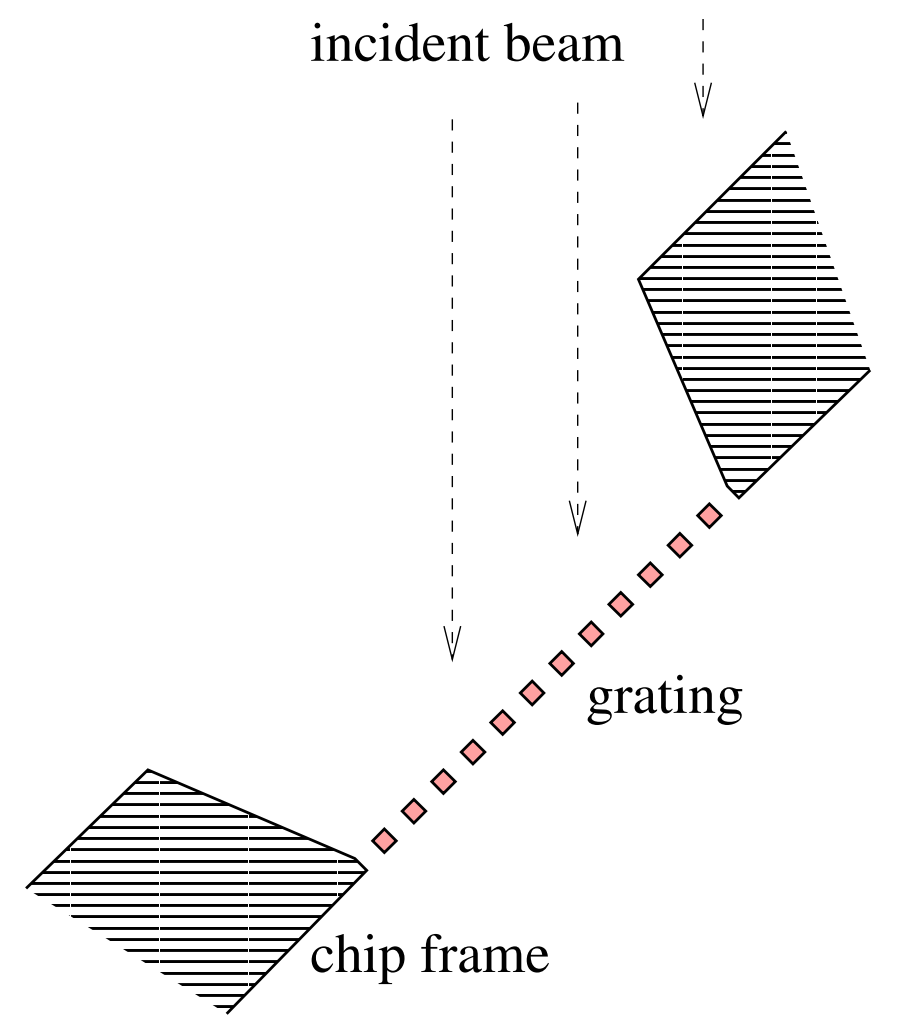

Figure 5.3: In the new transmission measurements to determine the grating bar geometry the centre of the incident beam spot has been placed on the centre of the grating to avoid a situation as shown schematically in the figure, where the transmission is artificially reduced at large rotation angles because parts of the beam are blocked by the frame of the grating. 
As a further improvement the zero angle position is no longer read from the dial that controls the grating rotation and that usually involves an offset but instead directly from the intensity profile as a function of the grating rotation angles which for this purpose newly includes negative angles. As a further convenience, the symmetry of the bars can be checked by comparing the left with the right-hand-side of the intensity profile, while the position of zero rotation is simply identified with the symmetry centre of the plot.

On the theoretical side, the purely geometrical description of the grating transmission as described in [9.9] has been abandoned in favour of a quantum mechanical treatment that includes the dispersion interaction of the atoms with the grating bars. It is reasonable to do this because it is known that the attraction of the atoms towards the grating bars leads to an effective narrowing of the slit, whereas the method to be improved aims at the ratio $\frac{t}{s}$ of the depth $t$ of the grating bars and the geometrical slit width $s$. Furthermore it has proven advantageous for experiment and theory to restrict the detection to the zeroth order diffraction maximum rather than widening the detector slit to gather the intensity of several low-order diffraction maxima at once.

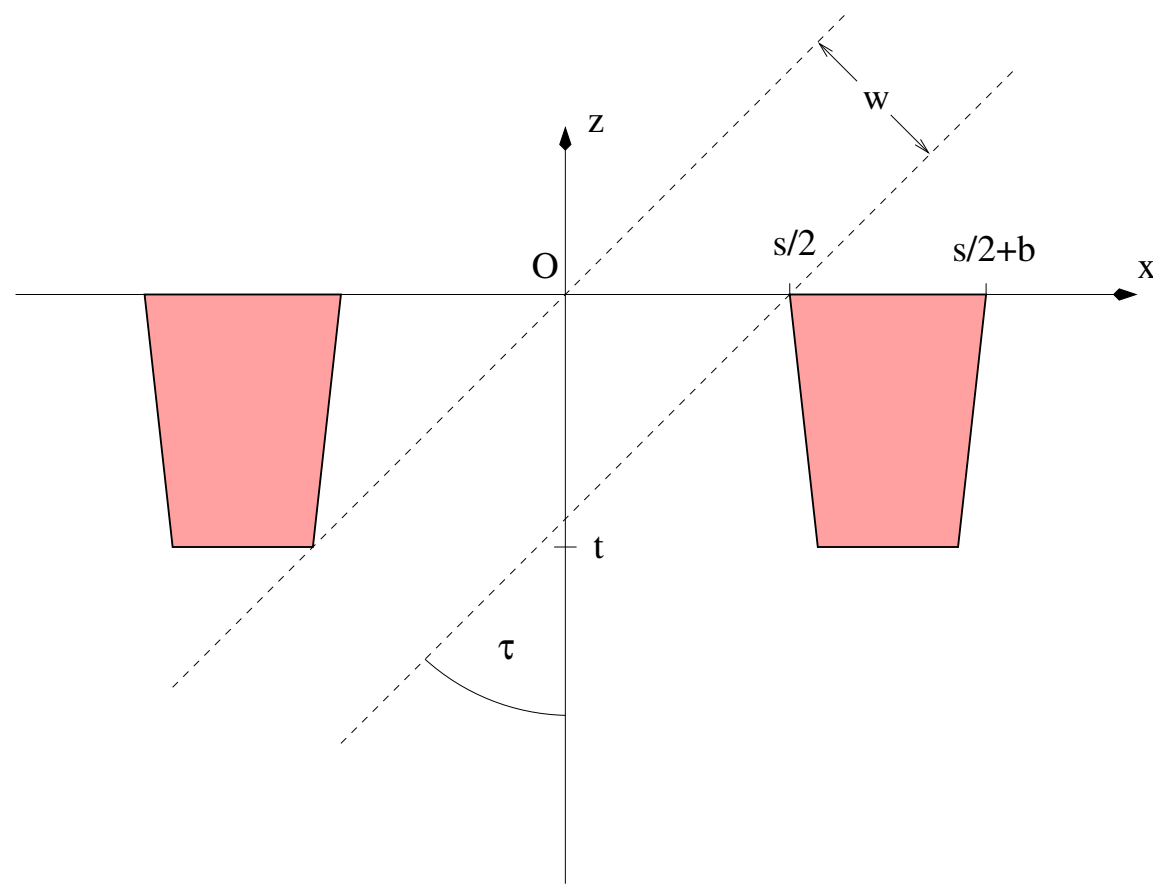

Figure 5.4: The effective geometrical slit width $w$ as seen in beam direction is reduced if the grating is rotated by $\tau$ with respect to the incident beam. For the new transmission measurements it has been taken into account that $w$ is further effectively reduced by the interaction of the atoms with the grating bars which changes with the grating rotation angle. 
For the zeroth order diffraction intensity $I_{\Omega}\left(\vartheta_{0}, \tau\right)$ as a function of the grating rotation angle $\tau$ it is sufficient to consider the Fraunhofer diffraction limit with due inclusion of the dispersion potential as developed in section 4.2.2. One has

$$
\frac{I_{\Omega}\left(\vartheta_{0}, \tau\right)}{I_{\Omega}\left(\vartheta_{0}, 0\right)} \propto\left|\int_{s_{1}(\tau)}^{s_{\mathrm{r}}(\tau)} \mathrm{d} x \mathrm{e}^{\mathrm{i} \varphi(x, \tau)}\right|^{2},
$$

where the angle-dependent boundaries $s_{1}(\tau)$ and $s_{\mathrm{r}}(\tau)$ of the illuminated region inside each grating slit are given by Eq. (4.41). For the quantum-mechanical phase $\varphi(x, \tau)$ one has

$$
\begin{aligned}
\varphi(x, \tau) & =\frac{C_{3}}{2 \hbar v \tan \alpha}\left\{\frac{1}{\zeta_{\mathrm{r}}^{2}}-\frac{1}{\left(\zeta_{\mathrm{r}}+t^{\prime} \tan \alpha\right)^{2}}\right\}+ \\
& -\frac{C_{9}}{8 \hbar v \tan \alpha}\left\{\frac{1}{\zeta_{\mathrm{r}}^{8}}-\frac{1}{\left(\zeta_{\mathrm{r}}+t^{\prime} \tan \alpha\right)^{8}}\right\}+ \\
& +\frac{C_{3}}{2 \hbar v \tan \gamma}\left\{\frac{1}{\zeta_{1}^{2}}-\frac{1}{\left(\zeta_{\mathrm{l}}+t^{\prime} \tan \gamma\right)^{2}}\right\}+ \\
& -\frac{C_{9}}{8 \hbar v \tan \gamma}\left\{\frac{1}{\zeta_{1}^{8}}-\frac{1}{\left(\zeta_{1}+t^{\prime} \tan \gamma\right)^{8}}\right\},
\end{aligned}
$$

with the impact parameters $\zeta_{1}$ and $\zeta_{\mathrm{r}}$ measured from the respective left and right boundary of the rotated slit given by Eq. (4.48) and Eq. (4.46). For brevity, the grating bars on each side of the single slit that needs to be calculated in the Fraunhofer limit have been assumed to be infinitely wide. The error in the resulting zeroth order intensity associated with this approximation amounts to less than $0.1 \%$. The angles $\alpha$ and $\gamma$, and the quantities $t^{\prime}$ and $b^{\prime}$ are given by Eq. (4.35) ff. They account for the beam trajectories passing the grating bars at different angles if the grating is rotated.

Without the atom-bar interaction the relative zeroth order diffraction intensity $\frac{I_{0}(\tau)}{I_{0}(0)}$ as a function of the grating rotation angle $\tau$ is given by

$$
\frac{I_{0}(\tau)}{I_{0}(0)}=\frac{w^{2}}{\cos ^{2} \tau s^{2}}
$$

where

$$
w:=\cos \tau\left(s_{\mathrm{r}}-s_{1}\right) \equiv \cos \tau\left\{\begin{aligned}
s & : \quad|\tau| \leq \beta \\
s-t(\tan |\tau|-\tan \beta) & : \quad \beta<|\tau|<\Theta_{0}
\end{aligned}\right.
$$

is the effective geometrical width of the slit upon grating rotation as seen in beam direction ( $z$ direction). At $|\tau|=\Theta_{0}$ the incident beam is completely blocked by the rotated grating (cf. Eq. (4.34)). The geometrical situation is illustrated in 


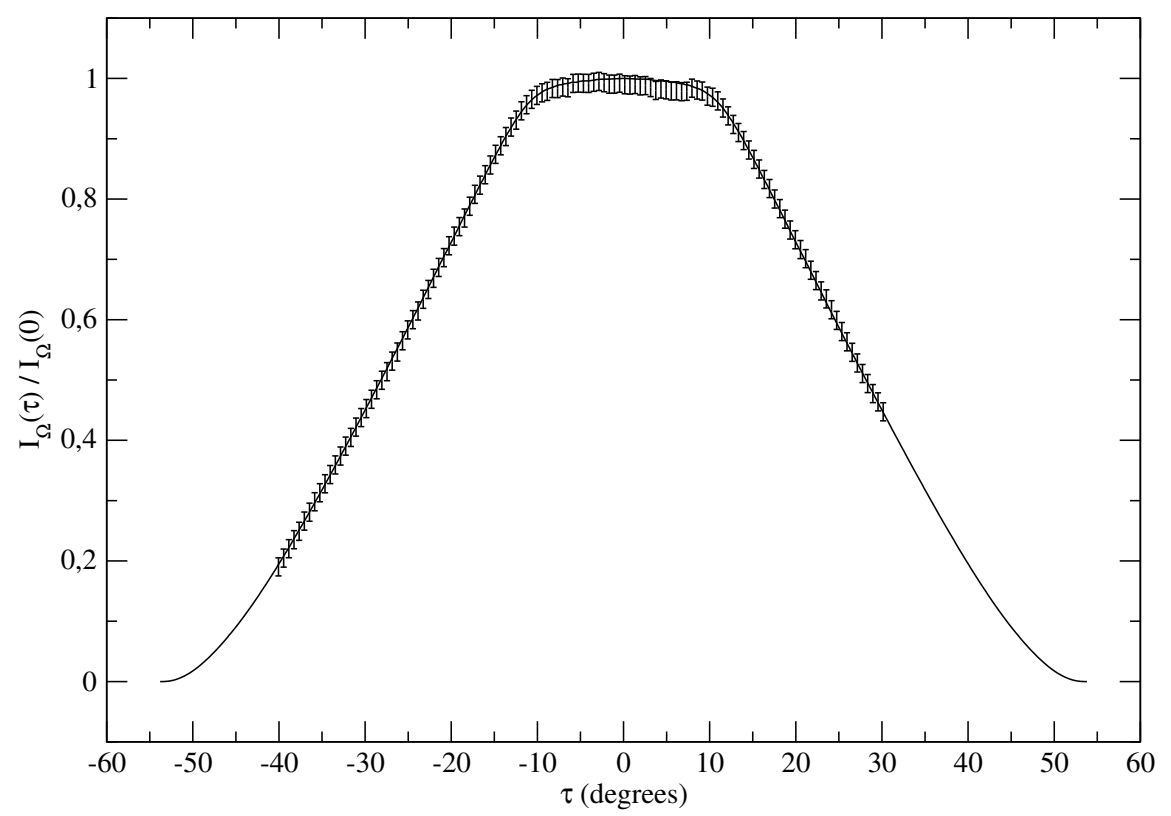

Figure 5.5: A measured curve of the intensity of the zeroth order maximum of groundstate helium atoms at $350 \mathrm{~K}\left(v=1904 \frac{\mathrm{m}}{\mathrm{s}}\right)$, diffracted from grating $5-3-1$, as a function of the grating rotation angle $\tau$ is fitted with a theoretical curve (solid line) according to Eq. (5.3). The two fit parameters are the ratio $\frac{t}{s}=0.853 \pm 0.008$ of the grating bar depth $t$ and the geometrical slit width $s$, and the wedge angle $\beta=(11 \pm 0.5)^{\circ}$ of the trapezoid-shaped grating bars.

Fig. 5.4. Note that in Eq. (5.5) another factor $\cos \tau$ appears because upon grating rotation more slits are illuminated for an incident beam of constant width. With inclusion of the atom-bar interaction the relative zeroth order diffraction intensity $\frac{I_{0}\left(\vartheta_{0}, \tau\right)}{I_{\Omega}\left(\vartheta_{0}, 0\right)}$ is found in excellent agreement with the measured intensity profile. Fig.5.5 shows a fit of Eq. (5.3) to an experimental transmission curve taken with the relevant grating 5-3-1 and a beam of ground-state He atoms. The dispersion interaction strength $C_{3}$ for $\mathrm{He}$ and the silicon nitride material of the grating is in the fit set equal to the theoretical value $C_{3}=0.136 \mathrm{meV} \mathrm{nm}^{3}$ calculated in chapter 3 . A $30 \%$ variation of $C_{3}$ does not significantly affect the resulting values of $\frac{t}{s}=0.85 \pm 0.01$ and the wedge angle $\beta=(11 \pm 0.5)^{\circ}$.

For the new generation of transmission gratings used in experiments today the purely geometrical method that ignores the atom-bar interaction has been abandoned in favour of the refined procedure as presented above. The old method can no longer be applied because the wedge angles of the new gratings are smaller than e.g. that of grating 5-3-1, and the grating bars are deeper as well. Fig. 5.6 shows that the theoretical predictions of the zeroth order intensity with or without atom-bar interaction differ considerably for a realistic example of a new grating 


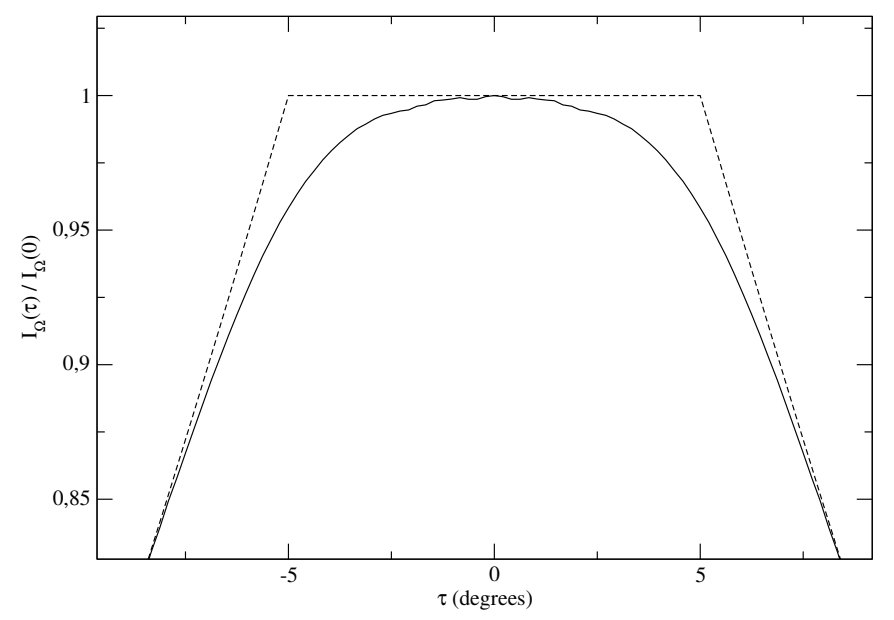

Figure 5.6: The inclusion of the atom-bar interaction is compulsory for an accurate determination of the wedge angle $\beta$ and the ratio of the bar depth $t$ and the slit width $s$ for the new gratings used in experiments today. Shown in the plot is a typical example with $\beta=5^{\circ}$ and $\frac{t}{s}=1.5$. The appropriate choice for a comparison with the experiment is the theoretical curve Eq. (5.3) that includes the atom-bar interaction (solid line) and that is qualitatively different from the theoretical curve without the interaction Eq. (5.5) (dashed line). As a result of the repulsive part of the atom-bar interaction the solid curve displays oscillations in the region around $\tau=0$, as will be discussed in section 5.2.

with $\beta=5^{\circ}$ and $\frac{t}{s}=1.5$. In particular, the region around $\tau=0$ is constant for the case without interaction, whereas the inclusion of the atom-bar interaction leads to a rounding-off where otherwise the edges at $\tau= \pm \beta$ would mark the wedge angle $\beta$.

\subsubsection{Slit Width}

The final requirement for the characterization of the grating geometry is to determine the slit width $s$. As the interaction of the particles with the grating bars effectively narrows the slit one faces the task of determining simultaneously the slit width $s$ and the strength $C_{3}$ of the attractive dispersion potential, whereby the narrowing of the slit can be supposed to increase with $C_{3}$ for intuitive reasons. This unwanted effect is reduced if weakly interacting particles are used, for which the best available choice are ground-state He atoms. An obvious and better alternative is to use X-rays which can be diffracted from the silicon nitride gratings [ 8 ] but, unfortunately, no such experiment has been performed so far. Another good alternative to measure the geometrical slit width $s$ is to acquire accurate scanning electronic micrographs of the rear side of the transmission gratings. From similar images it has recently been possible to even derive quantitative results for the 
roughness of the trailing edges of the single grating bars to an accuracy of a few Ångstrøm [100].

Such a direct route not being available a good way of finding the slit width $s$ circumventing the interdependence with the dispersion strength $C_{3}$ is to fit theoretically calculated diffraction intensities for ground-state He atoms to the corresponding experimental values with the slit width $s$ as a fit parameter, and adopting the theoretical value of $C_{3}=0.136 \mathrm{meV} \mathrm{nm}^{3}$ as calculated in chapter 3 . The $n$th order diffraction intensity $I_{\Omega}\left(\vartheta_{n}\right)$ is given by

$$
I_{\Omega}\left(\vartheta_{n}\right) \propto \mathrm{e}^{-\left(\frac{2 \pi n \sigma_{0}}{d}\right)^{2}} \int_{-\frac{s}{2}}^{\frac{s}{2}} \mathrm{~d} x^{\prime} \mathrm{e}^{-\mathrm{i} \kappa_{n} x^{\prime}} \mathrm{e}^{\mathrm{i} \varphi(x)},
$$

where the edge roughness $\sigma_{0}$ of the bars appears in a Debye-Waller-like exponential damping factor as explained earlier in section 4.3. The quantum mechanical phase $\varphi(x)$ hereby is adopted from Eq. (4.14). In particular, the wedge angle $\beta$ and the ratio $\frac{t}{s}$ of the grating bar depth $t$ and the slit width $s$ as determined in the previous section are contained in $\varphi(x)$ which is how they enter the fitting procedure as fixed parameters.

In accordance with Eq. (4.32) the relative intensities $\frac{I_{\Omega}\left(\vartheta_{n}\right)}{I_{\Omega}(0)}$ from Eq. (5.7) have been fitted to the relative areas $\frac{A(n)}{A(0)}$ of the $n$th order peaks extracted from a series of measured diffraction patterns of ground-state He atoms at various velocities $360.8 \frac{\mathrm{m}}{\mathrm{s}}<v<1904.7 \frac{\mathrm{m}}{\mathrm{s}}$ and grating $5-3-1$. Besides the slit width $s$ the edge roughness $\sigma_{0}$ has been used as a fit parameter. One obtains $\sigma_{0}=(1.6 \pm 0.1) \mathrm{nm}$ and $s=(66.7 \pm 0.2) \mathrm{nm}$ but it is possible that the value for the slit width $s$ contains a systematical error because there are qualitative discrepancies between the theoretical and the experimental diffraction intensities as is shown in Fig. 5.7. It is especially the theoretical third and sixth order intensities that deviate clearly from the measured values. This does not change if different values for the dispersion interaction strength $C_{3}$ are used and it is observed for diffraction patterns of various atoms and at all available velocities. In the past, the deviations have been attributed to the noise signal in the experiment which produces a constant background counting rate over all angles and affects most the low-intensity peaks because for them the signal-to-noise ratio is highest. However, in the new experiment now under consideration the background noise has been diligently identified and then subtracted from each measured intensity, so the origin of the discrepancies remains unclear.

In view of this unfavourable state of affairs the aim of this chapter need be extended to offer an explanation for the described differences between theory and experiment for ground-state He atom diffraction, furthermore, a reliable method of determining the slit width $s$ has to be given. This method has in particular to be proved independent of the effect that causes the discrepancies 


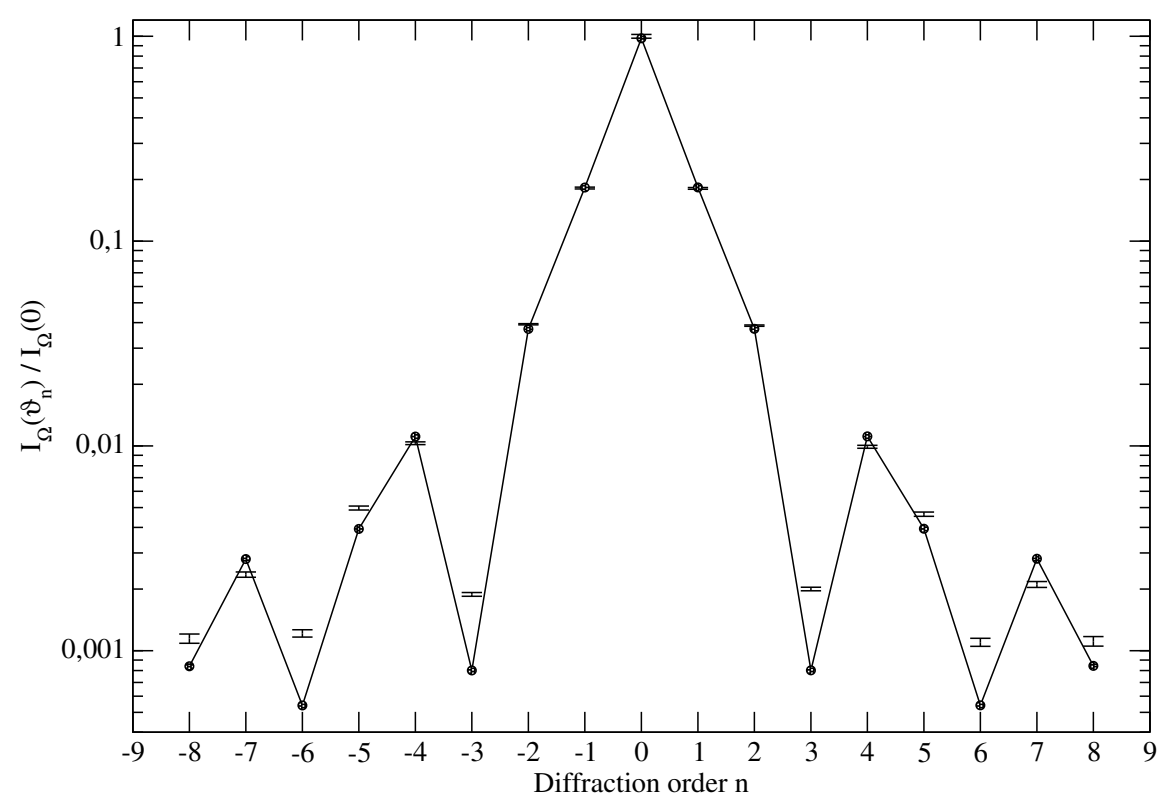

Figure 5.7: Experimental diffraction intensities of ground-state helium atoms at $v=$ $1769.9 \frac{\mathrm{m}}{\mathrm{s}}$ (data points with error bars) are directly fitted by Eq. (5.7) (full circles joined by a solid line). The theoretical dispersion interaction strength $C_{3}=0.136 \mathrm{meV} \mathrm{nm}^{3}$ and the grating geometry of grating 5-3-1 are fixed parameters. As fitted parameters one obtains the slit width $s=66.5 \mathrm{~nm}$ and the damping constant $\sigma_{0}=1.6 \mathrm{~nm}$. The fit shows systematic deviations especially at the $\pm 3 \mathrm{rd}$ and \pm 6 th order intensities. The discrepancies persist for all reasonable variations that both fixed and fit parameters can undergo.

between theoretical and experimental diffraction patterns. It turns out that these investigations lead to the prediction of a phenomenon which adds a new quality to the diffraction experiments currently in progress.

In order to check the value $s=66.7 \mathrm{~nm}$ of the slit width obtained above with the help of direct fits to the diffraction intensities a refined version of the method is presented in the following that has been employed by Grisenti et al. [12] for the simultaneous determination of the slit width $s$ and the dispersion interaction strength $C_{3}$ of ground-state atoms and a silicon nitride transmission grating. The technique is based on the Fraunhofer limit and can be applied to atom diffraction with the transmission grating normal parallel to the incident beam, i.e. no grating rotation, and the bars oriented vertically. Starting from Eq. (5.7), the essential step is to express the logarithm of the quantity

$$
\Phi( \pm \kappa):=\int_{0}^{\frac{s}{2}} \mathrm{~d} \zeta \mathrm{e}^{ \pm \mathrm{i} \kappa \zeta} \frac{\mathrm{d}}{\mathrm{d} \zeta} \mathrm{e}^{\mathrm{i} \varphi(\zeta)}
$$


in terms of the cumulant expansion [12]

$$
\ln \Phi( \pm \kappa)=\sum_{j=1}^{\infty} \frac{( \pm \mathrm{i} \kappa)^{j}}{j !} R_{j}
$$

where the quantum mechanical phase

$$
\varphi(\zeta):=\frac{C_{3}}{2 \hbar v \tan \beta}\left\{\frac{1}{\zeta^{2}}-\frac{1}{(\zeta+t \tan \beta)^{2}}\right\}
$$

is taken to arise only from the attractive dispersion interaction, neglecting the repulsive part. As grating rotations are not considered, the slit is symmetrical to its centre, so that the integral in Eq. (5.8) runs over half the slit width $s$, and the phase in Eq. (5.10) takes only account of the interaction of the atoms with the bar nearby. This yields for the relative intensities $I_{\Omega}\left(\vartheta_{n}\right)$ of the $n$th principal diffraction orders

$$
\frac{I_{\Omega}\left(\vartheta_{n}\right)}{I_{\Omega}\left(\vartheta_{0}\right)}=\frac{\mathrm{e}^{-\left(\frac{2 \pi n \sigma}{d}\right)^{2}}}{\left(\frac{\pi n}{d}\right)^{2}\left(s_{\mathrm{eff}}^{2}+\delta^{2}\right)}\left[\sin ^{2}\left(\frac{\pi n s_{\mathrm{eff}}}{d}\right)+\sinh ^{2}\left(\frac{\pi n \delta}{d}\right)\right] .
$$

Hereby the effective slit width

$$
s_{\text {eff }}:=s-2 \operatorname{Re}\left(R_{1}\right)=2 \operatorname{Re} \int_{0}^{\frac{s}{2}} \mathrm{~d} \zeta \mathrm{e}^{\mathrm{i} \varphi(\zeta)}<s
$$

reflects the fact that due to the attractive dispersion interaction between the atoms and the grating bars the detected intensity transmitted through the slits is effectively reduced because the attraction between the atoms and the bars leads to scattering at large angles while the detection is limited to small angles. The picture is not complete without the imaginary couterpart of Eq. (5.12) which defines the quantity $\delta$ as appears in Eq. (5.11). One has

$$
\delta:=2 \operatorname{Im}\left(R_{1}\right)=2 \operatorname{Im} \int_{0}^{\frac{s}{2}} \mathrm{~d} \zeta \mathrm{e}^{\mathrm{i} \varphi(\zeta)} .
$$

It is worth noting that with the definitions Eq. (5.12) and Eq. (5.13) it follows from the starting equation Eq. (5.7) that

$$
I_{\Omega}(0) \propto s_{\text {eff }}^{2}+\delta^{2} .
$$

The variable $\sigma$ in Eq. (5.11) is defined by

$$
\sigma^{2}:=\sigma_{0}^{2}+\operatorname{Re}\left(R_{2}\right)
$$


with the second cumulant coefficient given by

$$
R_{2}=\left(\frac{s}{2}\right)^{2}-R_{1}^{2}-2 \int_{0}^{\frac{s}{2}} \mathrm{~d} \zeta \zeta \mathrm{e}^{\mathrm{i} \varphi(\zeta)}
$$

and the grating bar edge roughness $\sigma_{0}$ as mentioned above. It is important to note that the cumulant expansion Eq. (5.9) is restricted to the first two terms in the sum which leads to the formula for the diffraction intensities Eq. (5.11). This is checked to be justified for ground-state He atoms by calculating the relative diffraction intensities from the starting equation Eq. (5.7) with the specific $V_{3^{-}}$ phase $\varphi(x)$ from Eq. (5.10) based on the dispersion potential $V_{3}(l)=-\frac{C_{3}}{l^{3}}$, and comparing them to the corresponding values as from Eq. (5.11), where $s_{\text {eff }}, \delta$, and $\sigma$ are calculated with Eq. (5.12) $f f$ on the basis of the $V_{3}$-phase. An example for such a comparison is shown in Fig. 5.8 for a typical velocity of the He beam whereby the geometrical parameters of the grating 5-3-1 have been adopted. It is evident from the figure that the restriction to two cumulant coefficients is applicable for diffraction orders up to 7.

In accordance with the procedure described in [12] the slit width $s$ of the particular grating 5-3-1, which is the one used for the diffraction of metastable atoms, is found by fitting Eq. (5.11) to the diffraction intensities of a series of 9 diffraction patterns of ground-state He atoms and grating 5-3-1 for 8 different velocities $360.8 \frac{\mathrm{m}}{\mathrm{s}}<v<1904.7 \frac{\mathrm{m}}{\mathrm{s}}$. Hereby $s_{\text {eff }}(v), \delta(v)$, and $\sigma(v)$ are treated as independent fit parameters, which rests on the assumption that for any attractive potential similarly weak as the dispersion potential $V_{3}$, the cumulant expansion Eq. (5.9) practically converges after the second term. Technically, the zeroth order intensity in the experiment is known to unwantedly arise also from He clusters in the beam and is left out of the fit. Fig. 5.9 illustrates on the basis of a typical example that the experimental diffraction intensities are well fitted by Eq. (5.11).

The slit width $s$ is obtained in the next step by fitting to the experimental dataset $s_{\text {eff }}(v)$ a corresponding theoretical curve from Eq. (5.12) which is calculated with the specific $V_{3}$-phase, where the slit width $s$ and the dispersion interaction strength $C_{3}$ serve as fit parameters, and $v$ is inserted according to the experimental beam velocities. At this point it turns out that the experimentally obtained values of $s_{\text {eff }}(v)$ and $\delta(v)$ are impossible to match as respective real and imaginary parts of the integral $\int_{0}^{s / 2} \mathrm{~d} \zeta \mathrm{e}^{\mathrm{i} \varphi(\zeta)}$ if $\varphi(\zeta)$ is taken to arise from a dispersion potential of the form $V(l)=-\frac{C_{3}}{l^{3}}$. In particular, the theoretical value of $\delta \approx 1.2 \mathrm{~nm}$ is less than half as large as that extracted from the experiment $(\delta \approx 3 \mathrm{~nm})$. The interested reader may check the figure captions of Fig. 5.8 and Fig. 5.9 which are comparable in this sense.

The question arises whether it is justified to ignore the mismatch of $\delta$ and proceed by focusing on the effective slit width $s_{\text {eff }}$ alone in order to obtain the 


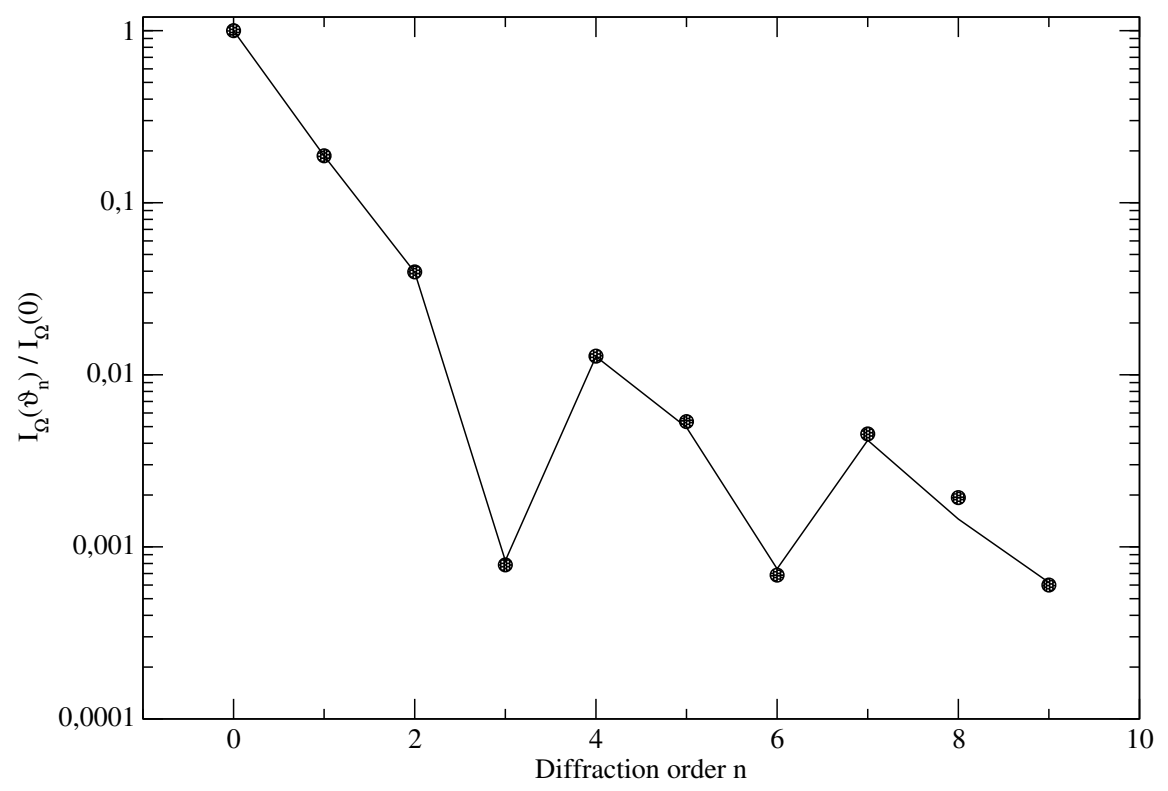

Figure 5.8: The principal order diffraction intensities of ground state He at $v=$ $1769.9 \frac{\mathrm{m}}{\mathrm{s}}$ calculated with the exact theory (full circles) are in excellent agreement with those calculated with the help of the cumulant expansion [I2]. For the sake of clarity, the latter data points are merely indicated by a solid line joining them. With the given velocity the effective slit width becomes $s_{\text {eff }}=65.2 \mathrm{~nm}$ which is $1.5 \mathrm{~nm}$ less than the assumed geometrical slit width $s=66.7 \mathrm{~nm}$. The difference $s-s_{\text {eff }}$ is generally of the same size as the parameter $\delta=1.2 \mathrm{~nm}$. With an edge roughness of $\sigma_{0}=1.6 \mathrm{~nm}$ the calculation further yields $\sigma=2.6 \mathrm{~nm}$.

slit width $s$. Equivalently, one may ask whether the parameters $s_{\text {eff }}$ and $\delta$ are decoupled in the fit. Looking at Eq. (5.11) is becomes evident that for typical values of the parameters $s_{\text {eff }} \approx 65 \mathrm{~nm}$ and $\delta \approx 3 \mathrm{~nm}$ their influence on the intensity pattern does tend to decouple. Fig. 5.10 illustrates that the third and sixth order intensity are dominated by the $\sinh ^{2}$ term in Eq. (5.11) whose argument is essentially $\delta$, while the other intensities are dominated by the $\sin ^{2}$ term which contains $s_{\text {eff }}$.

It is thus obvious that the discrepancies between theory and experiment for the third and sixth order intensities that at the beginning of this section motivated the current investigation are now reflected in the large experimental values of $\delta$. Neither the large values of $\delta$ nor the partial mismatch of the direct fit to the measured diffraction intensities can be explained within the employed concept of the $V_{3}$ dispersion potential and the assumed grating geometry. In the case of the direct fit one has to hope that the discrepancies of the third and sixth diffraction order intensities do not have a negative influence on the fit that would lead to a wrongly determined slit width $s$ which is not acceptable in view of the desired 


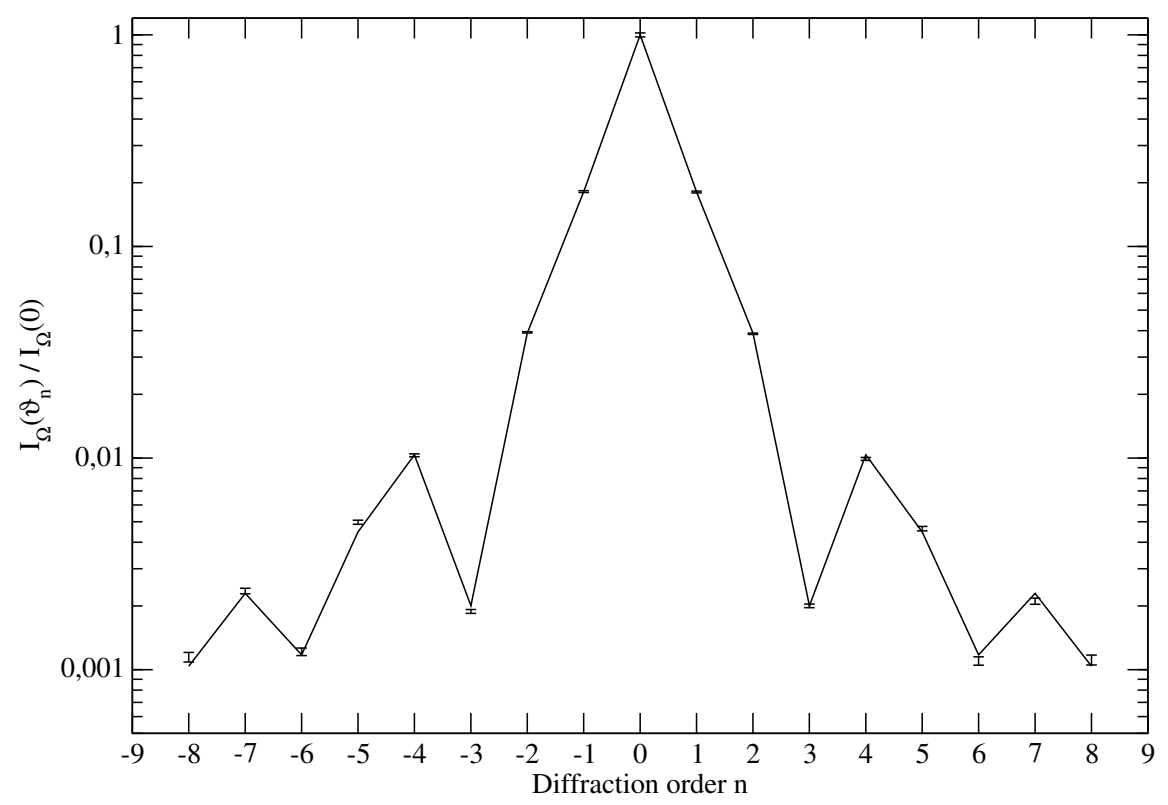

Figure 5.9: The relative peak areas $\frac{A(n)}{A(0)}$ as extracted from an experimental diffraction pattern of He at $v=1769.9 \frac{\mathrm{m}}{\mathrm{s}}$ with grating 5-3-1 (data points with error bars) are well fitted by the cumulant-based formula Eq. (5.11) (solid line) with three independent fit parameters $s_{\text {eff }}=65.6 \mathrm{~nm}, \delta=3.0 \mathrm{~nm}$, and $\sigma=2.4 \mathrm{~nm}$. These parameters can be compared to those that in Fig. 5.8 help to fit theoretical diffraction intensities for an analogous situation.

$1 \%$ accuracy of $s$.

On the other hand, for the $s_{\text {eff }}$ method it has become apparent that the features in the measured diffraction pattern which might arise from an unknown difference between the real and the theoretical potential, can be absorbed largely during a fit by the parameter $\delta$ while the important other parameter $s_{\text {eff }}$ which will give the slit width $s$ is expected to remain unchanged. In order to specify the last point the measured third and sixth order intensities which most distinctly mark the difference between the real and the theoretically assumed potential are artificially varied by up to $50 \%$ and then the fit of Eq. (5.11) is repeated. In the course of this numerical experiment the fitted value of $s_{\text {eff }}$ varies by less than $0.1 \mathrm{~nm}$ while the values of $\delta$ are seen to follow the change in the intensities. From this one may conclude that despite the discrepancies it is justified to compare in a fitting procedure the values of $s_{\text {eff }}$ that have been extracted from the experiment to those calculated on the basis of the given grating geometry and the $V_{3}$ atom-bar potential.

The slit width $s$ of the grating 5-3-1 is obtained by an extrapolation to very large velocities $v \rightarrow \infty$ of the experimentally found dataset of $s_{\text {eff }}(v)$ where $v$ stands for the beam velocities of the various diffraction patterns from each of 


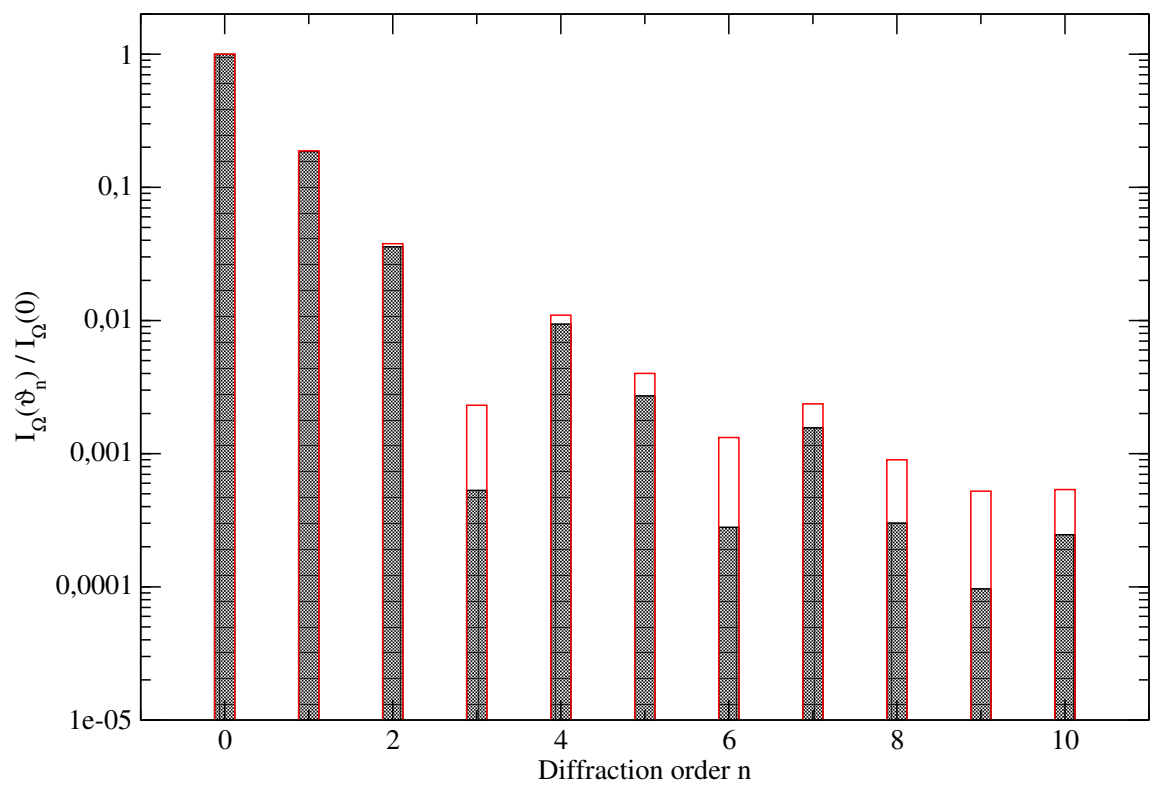

Figure 5.10: The theoretical relative $n$th order diffraction intensities of He and a grating like 5-3-1 have been calculated with the cumulant-based formula Eq. (5.11) and are represented by the total height of the bars shown in the plot. The absolute contribution of the $\sinh ^{2}$ term is equal to the open top section of the bars whereas the shaded section represents the contribution of the $\sin ^{2}$ term. It can be seen that the $3 \mathrm{rd}$ and 6 th order intensities are dominated by the former term which contains the parameter $\delta$.

which one data point $s_{\text {eff }}(v)$ is extracted. The meaning of the limit $v \rightarrow \infty$ is that the influence of the atom-bar interaction as given by Eq. (5.10) vanishes as $v$ appears in the denominator. It has proven justified above to perform the extrapolation with a corresponding theoretical curve that is based on the usual dispersion potential $V(l)=-\frac{C_{3}}{l^{3}}$, and the grating geometry as determined earlier. In particular, the assumption of straight rather than corrugated bar edges is maintained as it has been shown in section 4.3 that the edge roughness in lowest order leads only to a damping of the outer maxima in the diffraction pattern by which the effective slit width $s_{\text {eff }}$ is not affected, although one should bear in mind that the slit width $s$ to be determined represents an average over all illuminated slits and the vertical coherence length $L_{y}$ of the incident particles.

G.C. Hegerfeldt has pointed out [10]] that the theoretical effective slit width $s_{\text {eff }}$ as plotted versus the inverse of the square root $\frac{1}{\sqrt{v}}$ of the beam velocity $v$ is well approximated by

$$
s_{\mathrm{eff}}\left(\frac{1}{\sqrt{v}}\right)=s-K \frac{1}{\sqrt{v}},
$$

hence the data points $s_{\text {eff }}\left(\frac{1}{\sqrt{v}}\right)$ are expected to fall on a straight line, whose 
intersection with the ordinate gives the slit width $s$ while the slope

$$
K:=\sqrt{\frac{\pi C_{3}}{\hbar \tan \beta}}
$$

helps to determine the dispersion interaction strength $C_{3}$. This method which is in the following referred to by "the $s_{\text {eff }}\left(\frac{1}{\sqrt{v}}\right)$ method" is easier to handle and

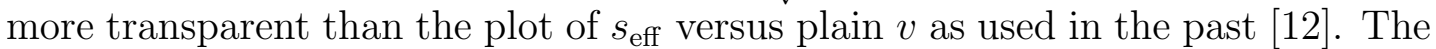
derivation of the simple result Eq. (5.17) starts from the definition Eq. (5.12) of the effective slit width $s_{\text {eff }}$ which can be written as

$$
s_{\text {eff }}=2 \int_{0}^{\frac{s}{2}} \mathrm{~d} \zeta \cos \left[\frac{C_{3}}{2 \hbar v \tan \beta}\left(\frac{1}{\zeta^{2}}-\frac{1}{(\zeta+t \tan \beta)^{2}}\right)\right] .
$$

The second argument

$$
y(\zeta):=\frac{C_{3}}{2 \hbar v \tan \beta} \frac{1}{(\zeta+t \tan \beta)^{2}}
$$

of the cosine in Eq. (5.19) is smaller than the first argument

$$
x(\zeta):=\frac{C_{3}}{2 \hbar v \tan \beta} \frac{1}{\zeta^{2}} .
$$

For $\operatorname{SiN}_{x}$ grating bars with a realistic depth of $t=50 \mathrm{~nm}$ and a wedge angle $\beta=11^{\circ}$, passed by a beam of ground-state helium atoms at a velocity $v \geq 500 \frac{\mathrm{m}}{\mathrm{s}}$ one has

$$
y(\zeta) \leq y(0)=\frac{C_{3}}{2 \hbar v \tan \beta} \frac{1}{(t \tan \beta)^{2}} \leq 0.01
$$

With a beam of metastable atoms whose dispersion interaction strength $C_{3}$ is up to 50 times stronger than that of ground-state helium atoms the number on the right hand side of Eq. (5.22) rises proportionally. A smaller wedge angle $\beta$ is seen to also increase the maximum value of $y(\zeta)$. As long as $\beta>5^{\circ}$ it is guaranteed even for metastable atoms that $y(\zeta) \leq 1$ so that the influence of $y(\zeta)$ as part of the argument in Eq. (5.19) always amounts to less than half a cycle of the cosine. In order to find an appropriate approximation one deduces from Eq. (5.19)

$$
s_{\text {eff }}=2 \int_{0}^{\frac{s}{2}} \mathrm{~d} \zeta\{\cos [x(\zeta)] \cos [y(\zeta)]+\sin [x(\zeta)] \sin [y(\zeta)]\} .
$$

For ground-state He atoms it follows from Eq. (5.22) that $y(\zeta)$ can be replaced by zero in Eq. (5.23). The error in $s_{\text {eff }}$ associated with this approximation turns out to be less than $0.1 \mathrm{~nm}$. Therefore one has

$$
s_{\text {eff }}=2 \int_{0}^{\frac{s}{2}} \mathrm{~d} \zeta \cos [x(\zeta)]
$$


which can be solved analytically with the substitution $\xi:=x(\zeta)$ as $x(\zeta)$ is a strictly monotonous function of $\zeta$. This yields

$$
s_{\text {eff }}=\sqrt{A} \int_{\frac{4 A}{s^{2}}}^{\infty} \mathrm{d} \xi \frac{\cos \xi}{\xi^{\frac{3}{2}}},
$$

where, for convenience, $A:=\frac{C_{3}}{2 \hbar v \tan \beta}$ has been defined. On integrating by parts and using the definite integral [176]

$$
\int_{0}^{\infty} \mathrm{d} z \frac{\sin (z)}{z^{s}}=\frac{\pi}{2 \Gamma(z) \sin \left(\frac{\pi s}{2}\right)}, \quad 0<s<2
$$

one finds

$$
\begin{aligned}
& s_{\text {eff }}=\sqrt{A}\left\{\left[\frac{-2 \cos (\xi)}{\sqrt{(\xi)}}\right]_{\frac{4 A}{s^{2}}}^{\infty}-2\left(\int_{0}^{\infty} \mathrm{d} \xi \frac{\sin (\xi)}{\sqrt{(\xi)}}-\int_{0}^{\frac{4 A}{s^{2}}} \mathrm{~d} \xi \frac{\sin (\xi)}{\sqrt{(\xi)}}\right)\right\}= \\
& =s-\sqrt{2 \pi A} \equiv s-K \frac{1}{\sqrt{v}},
\end{aligned}
$$

where in Eq. (5.28) the integral $\int_{0}^{\frac{4 A}{s^{2}}}$ has been neglected because its value amounts to less than $3 \%$ of the other integral, which corresponds to a correction to $s_{\text {eff }}$ of less than $0.1 \mathrm{~nm}$.

Fig. 5.11 illustrates the application of the $s_{\text {eff }}\left(\frac{1}{\sqrt{v}}\right)$ method for ground-state He atoms diffracted from grating 5-3-1. It is apparent that the experimental data points are distributed along a straight line, in agreement with the theoretical concept. The line indicated in the figure is obtained by a least-square-fit of Eq. (5.17) to the data. The slit width $s$ comes out of the fit as $s=(66.8 \pm 0.2) \mathrm{nm}$ which agrees with the value $s=(66.7 \pm 0.2) \mathrm{nm}$ that has been obtained earlier from a direct fit of theoretically calculated diffraction intensities to the experiment, whereby the theoretically calculated value for the dispersion interaction strength $C_{3}^{\text {theo }}=0.136 \mathrm{meV} \mathrm{nm}^{3}$ has been used as a fixed parameter.

The slope of the line that fits the experimental data points $s_{\text {eff }}\left(\frac{1}{\sqrt{v}}\right)$ in Fig. 5.11 comes out of the fit as $K=(-48 \pm 7) \mathrm{nm} \sqrt{\frac{\mathrm{m}}{\mathrm{s}}}$. If this value is interpreted according to Eq. (5.18) in terms of the dispersion strength one finds $C_{3}=(0.09 \pm$ $0.3) \mathrm{meV} \mathrm{nm}^{3}$. In similar measurements with ground-state He atoms but with different gratings it has for longer been a puzzle that the resulting values of $C_{3}$ are varying between $0.05 \mathrm{meV} \mathrm{nm}^{3}$ and $0.16 \mathrm{meV} \mathrm{nm}^{3}$. A possible explanation for this unusual behaviour is that the He atoms do not experience the total wedge angle $\beta$ of the bar but rather a local wedge angle $\beta^{\text {loc }}$ which describes the average 


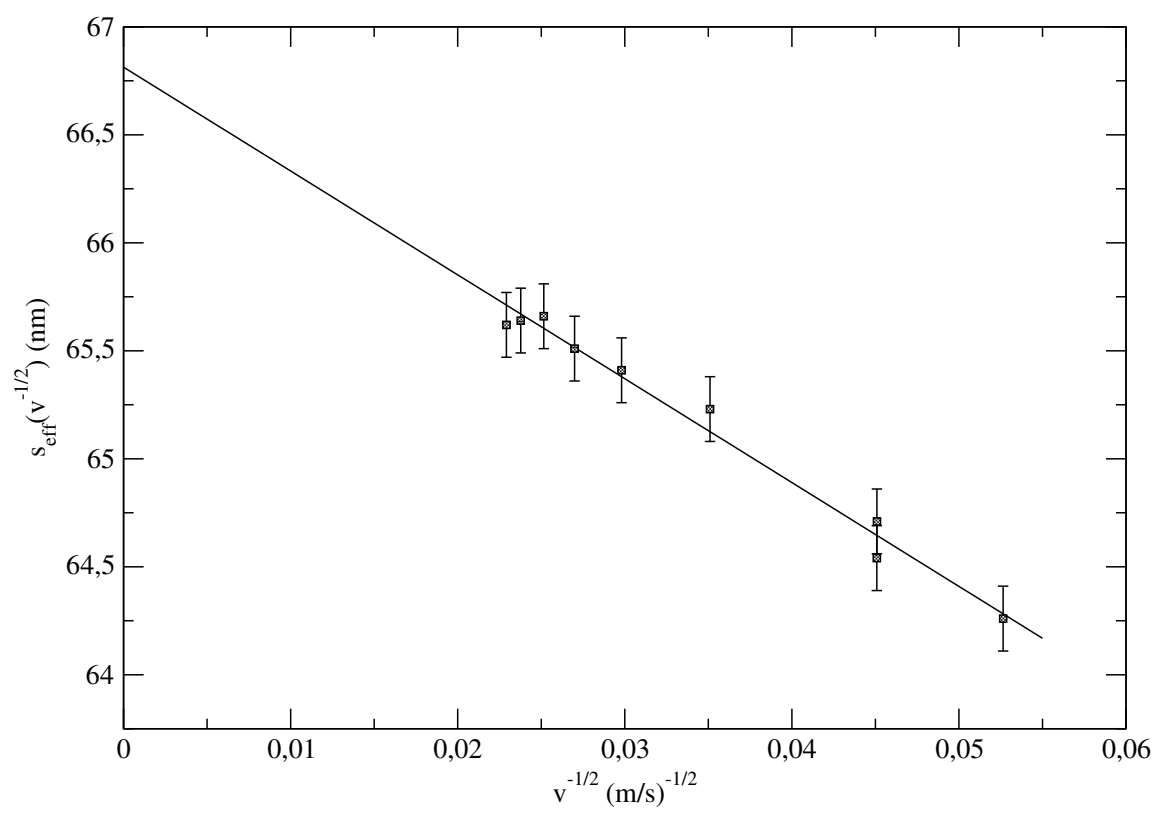

Figure 5.11: The values of the effective slit width $s_{\text {eff }}$ as a function of the inverse of the square root of the velocity $v$ as determined by fitting Eq. (5.11) to measured diffraction intensities at several $v$ fall on a straight line as predicted by Eq. (5.17). The errors are estimated from those of the respective fits. By extrapolating the straight line, which is the result of a two-parameter least-square fit of Eq. (5.17), towards the ordinate one finds the geometrical slit width $s=(66.8 \pm 0.2) \mathrm{nm}$. From the slope $K=(-48 \pm 7) \mathrm{nm} \sqrt{\frac{\mathrm{m}}{\mathrm{s}}}$ of the line the dispersion strength $C_{3}$ can be determined (see text).

shape of that protruding portion of the bar side, with which the atoms are actually in contact during their passage through the slit. This hypothesis implies that the real shape of the horizontal cross-section through the grating bars deviates from an exact trapezoid in that the sides are slightly concave, i.e. bent inwards (cf. Fig. 5.12). Considering the fact that the grating bars are the result of an etching process the notion of concave bar sides appears plausible.

For ground-state atoms whose dispersion interaction with the bar material is comparatively weak and short-ranged any part near the narrow face of the bar that is further away from a given trajectory than a certain distance of a few nanometers can be neglected in good approximation. This is the reason why the depth $t$ of the grating bars has dropped out of the formula Eq. (5.28) for the effective slit width $s_{\text {eff }}$ of grating 5-3-1 with ground-state He atoms. A numerical experiment with theoretical diffraction patterns of He from a virtual grating 5-3-1 reveals that only a $10 \mathrm{~nm}$-thick slice at the foot of the grating bars bears any relevance for the diffraction, while the total bar depth is $t=57 \mathrm{~nm}$ (cf. 
Fig.5.12). For example, the value of the slope $K \equiv \sqrt{\frac{\pi C_{3}}{\hbar \tan \beta}}$ just determined for ground-state He atoms and grating 5-3-1 is consistent with the theoretical value $C_{3}=0.136 \mathrm{meV} \mathrm{nm}^{3}$ of the dispersion interaction strength for He and $\mathrm{SiN}_{x}$ if the wedge angle $\beta=11.0^{\circ}$ is replaced by $\beta^{\text {loc }}=15.7^{\circ}$. This suggests a shape similar to that shown in Fig. 5.12.

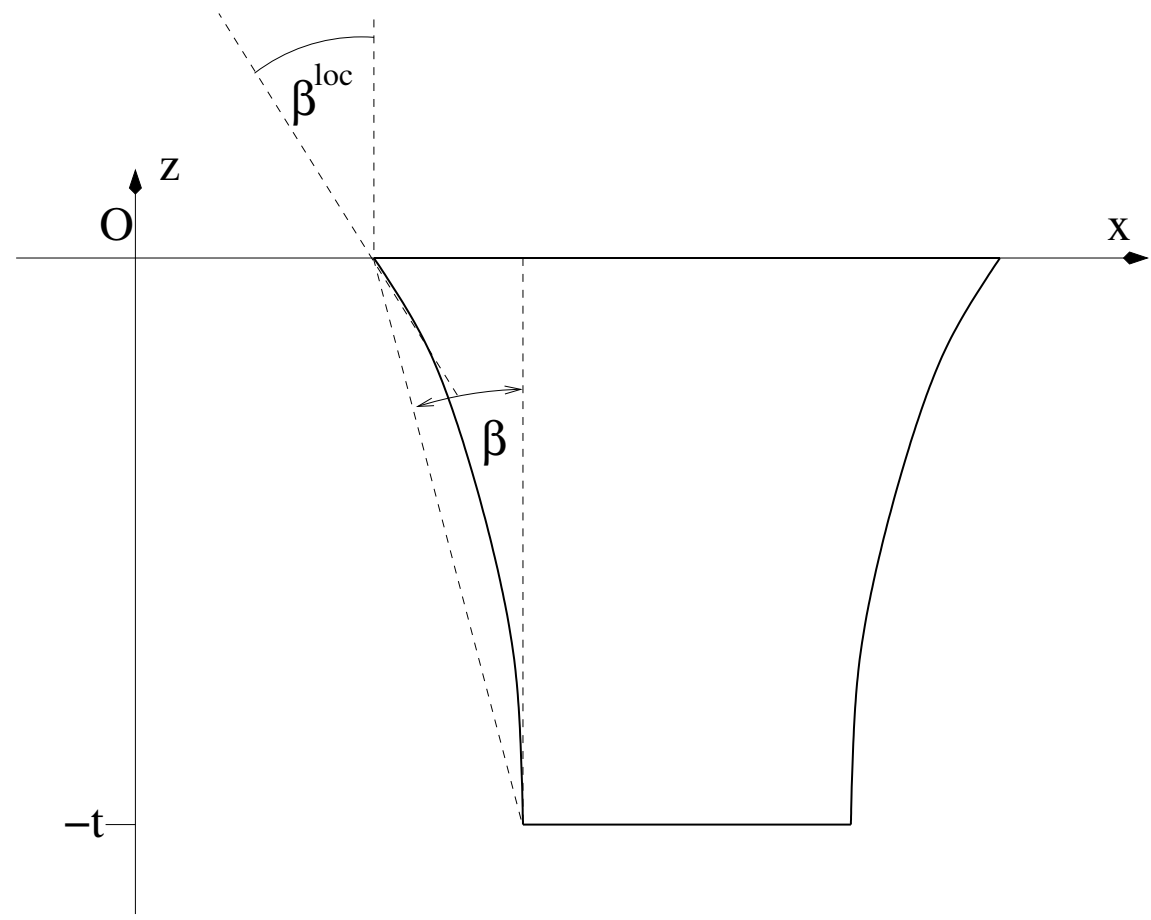

Figure 5.12: As the grating bars are the result of an etching process during the manufacturing it is probable that the sides of the bar cross-section shown in the figure deviate from the idealization of a straight line (dotted line on the left of the bar). Experimental evidence suggests that the cross-section rather has concave sides as indicated in the figure, which leads to the concept of a local wedge angle $\beta^{\text {loc }}$ especially at the bottom of the bar $(z=0)$. The protruding edge there in place interacts most with the atomic beam that passes the bar and thus determines the diffraction rather than the averaged overall wedge angle $\beta$.

It is clear that during the measurement of the transmitted zeroth order intensity $I_{\Omega}\left(\vartheta_{0}, \tau\right)$ as a function of the grating rotation angle $\tau$ the slightly concave region of the bar sides is hidden behind the protruding top and foot edges so that the total wedge angle $\beta$ determined by this experiment actually describes the angle of a straight line between the top and foot edge of the bar with respect to the grating normal. As the supposed bar sides bend away from the atomic trajectories it can be expected that the dispersion interaction appear somewhat reduced in the transmission experiment. This has been qualitatively confirmed in a series 
of attempts to simultaneously adjust the ratio $\frac{t}{s}$ of the bar depth $t$ and the slit width $s$, the total wedge angle $\beta$, and the dispersion interaction strength $C_{3}$ in a fit of Eq. (5.3) to the measured intensity profile $\frac{I_{\Omega}\left(\vartheta_{0}, \tau\right)}{I_{\Omega}\left(\vartheta_{0}, 0\right)}$. With due caution because of a quite shallow minimum of the sum of squared deviations in this type of fit the dispersion interaction strength was found to be $C_{3}=(0.1 \pm 0.02) \mathrm{meV} \mathrm{nm}^{3}$ which is smaller than the theoretical value $C_{3}=0.136 \mathrm{meV} \mathrm{nm}^{3}$ and in agreement with the hypothesis of slightly concave bar sides.

Another check of the hypothesis is to apply the $s_{\text {eff }}\left(\frac{1}{\sqrt{v}}\right)$ method to different atomic species diffracted from the same grating. The best choice is to use ground-state $\mathrm{He}$ atoms and $\mathrm{D}_{2}$ molecules because they have virtually the same mass ( $m_{\mathrm{He}}=4.002602$ a.m.u., $m_{\mathrm{D} 2}=4.048204$ a.m.u.) so that beams of the two species have about the same velocity for a given beam source temperature. In the diffraction experiments of the present type the internal degrees of freedom of the $\mathrm{D}_{2}$ molecules can be neglected and the particles can be treated like atoms. As the only relevant difference between $\mathrm{He}$ and $\mathrm{D}_{2}$ remains that the latter attains a dispersion interaction strength $C_{3}$ with silicon nitride which is three times as large as that of He atoms.

If the bar cross-section were an exact trapezoid one would expect from the $s_{\text {eff }}\left(\frac{1}{\sqrt{v}}\right)$ method when applied to both species that the values of the determined slopes $K$ be subject to

$$
\frac{K^{2}(\mathrm{He})}{K^{2}\left(\mathrm{D}_{2}\right)}=\frac{C_{3}(\mathrm{He})}{C_{3}\left(\mathrm{D}_{2}\right)},
$$

as follows from Eq. (5.18). Fig. 5.13 displays the result of an examination of diffraction patterns of ground-state He atoms and $\mathrm{D}_{2}$ molecules with grating 52-2. By least-square-fits of straight lines to the respective datasets $s_{\text {eff }}\left(\frac{1}{\sqrt{v}}\right)$ of He and $\mathrm{D}_{2}$ one obtains for the slit width $s=(70.5 \pm 0.2) \mathrm{nm}(\mathrm{He})$ and $s=$ $(70.7 \pm 0.2) \mathrm{nm}\left(\mathrm{D}_{2}\right)$ which is satisfactorily consistent. For the slopes in the previously used units one has $K=(-37 \pm 5)(\mathrm{He})$ and $K=(-73 \pm 10)\left(\mathrm{D}_{2}\right)$. A comparison with the ratio of theoretical values for the dispersion interaction strengths $C_{3}$, like Eq. (5.29) yields

$$
0.257=\frac{K^{2}(\mathrm{He})}{K^{2}\left(\mathrm{D}_{2}\right)}<\frac{C_{3}(\mathrm{He})}{C_{3}\left(\mathrm{D}_{2}\right)}=0.330
$$

which is in disagreement with the theoretically expected equality of both ratios for an exact trapezoid shape of the bars. Assuming concave bar sides and the theoretical values of $C_{3}$ to be correct, the fitted slopes $K$ in Fig. 5.13 are consistent with locally averaged wedge angles $\beta^{\text {loc }}=25.4^{\circ}(\mathrm{He})$ and $\beta^{\text {loc }}=20.3^{\circ}$ $\left(D_{2}\right)$ while the total wedge angle of grating $5-2-2$ is about $\beta=13.0^{\circ}$. This result is intuitively correct because due to their larger dispersion interaction strength $C_{3}$ the $\mathrm{D}_{2}$ molecules are sensitive to a larger portion of the bar side which on average is more parallel to the beam trajectory, i.e. closer to the total wedge 


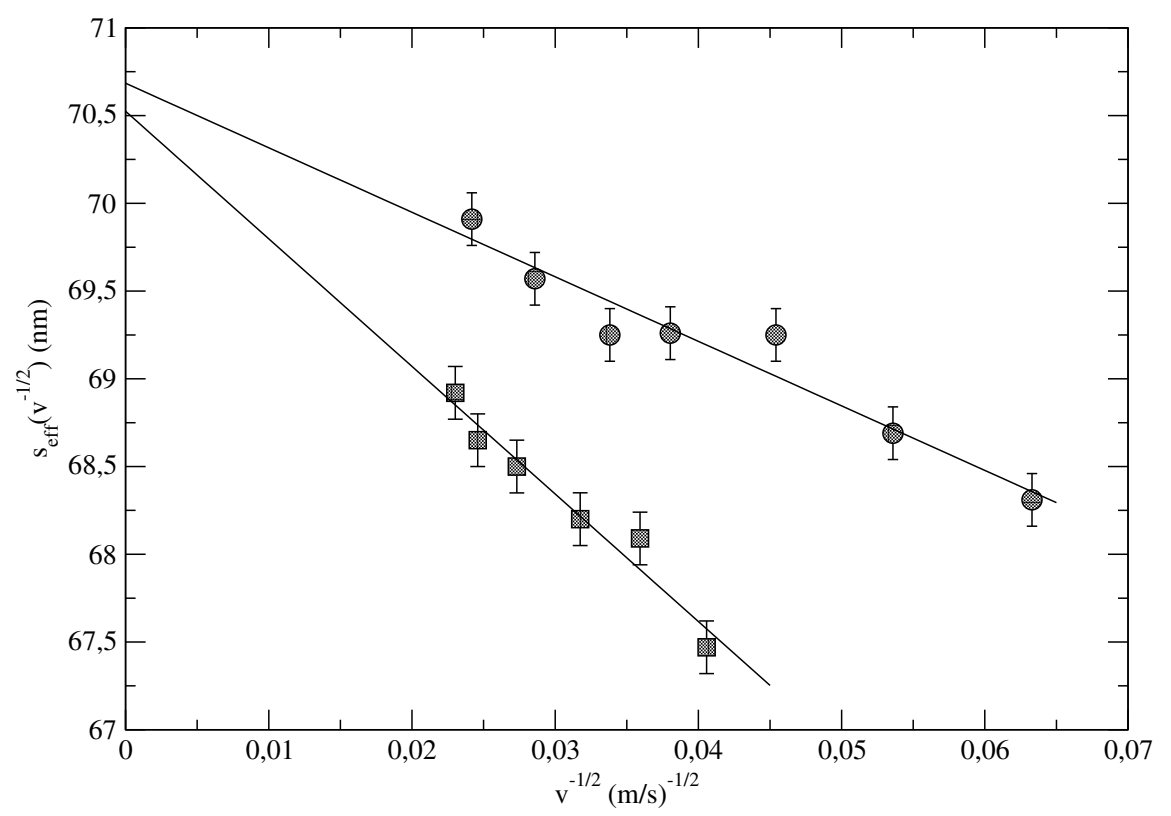

Figure 5.13: From diffraction experiments with grating 5-2-2 [ए5] the values of $s_{\text {eff }}\left(\frac{1}{\sqrt{v}}\right)$ for ground-state helium (full circles) and $\mathrm{D}_{2}$ (full squares) are extracted and fitted with straight lines according to Eq. (5.17). The result for the geometrical slit width $s=(70.7 \pm 0.2) \mathrm{nm}(\mathrm{He})$ agrees well with that for $\mathrm{D}_{2}: s=(70.5 \pm 0.2) \mathrm{nm}$. From the measured slopes $(-37 \pm 5) 10^{-9} \sqrt{\mathrm{ms}}(\mathrm{He})$ and $(-73 \pm 10) 10^{-9} \sqrt{\mathrm{ms}}\left(\mathrm{D}_{2}\right)$ conclusions on the wedge angle of the grating bars can be drawn.

angle $\beta=13.0^{\circ}$. The average local wedge angle $\beta^{\text {loc }}=20.3^{\circ}$ for $\mathrm{D}_{2}$ molecules is therefore expected to be smaller than that of He atoms which are sensitive only to the very foot of the bar where the local wedge angle $\beta^{\text {loc }}=25.4^{\circ}$ is largest.

These considerations show that the method of determining the slit width $s$ of grating 5-3-1 by direct fits of theoretical to experimental diffraction intensities, which stands at the beginning of this section, is in fact not consistent with the $s_{\text {eff }}\left(\frac{1}{\sqrt{v}}\right)$ method despite the similar results for $s$. The reason is that the direct fit is based on an ideal trapezoid bar cross-section with an overall wedge angle $\beta=11^{\circ}$ whereas the $s_{\text {eff }}\left(\frac{1}{\sqrt{v}}\right)$ method in the previous paragraph indicates that the diffraction of helium atoms is determined by a local wedge angle $\beta^{\text {loc }}=15.7^{\circ}$ near the foot of each bar. It turns out that during the fit the systematic mismatch of the third and sixth diffraction order intensities leads to an error that cancels with that arising from the undue use of the overall wedge angle instead of the appropriate local wedge angle $\beta^{\text {loc }}=15.7^{\circ}$ of grating 5 -3-1 for helium atoms

In order to illustrate this coincidence the third and sixth order are assigned a reduced weight during a new fit while the local wedge angle $\beta^{\text {loc }}=15.7^{\circ}$ is used to replace the formerly employed total wedge angle $\beta=11^{\circ}$ as a fixed parameter. 


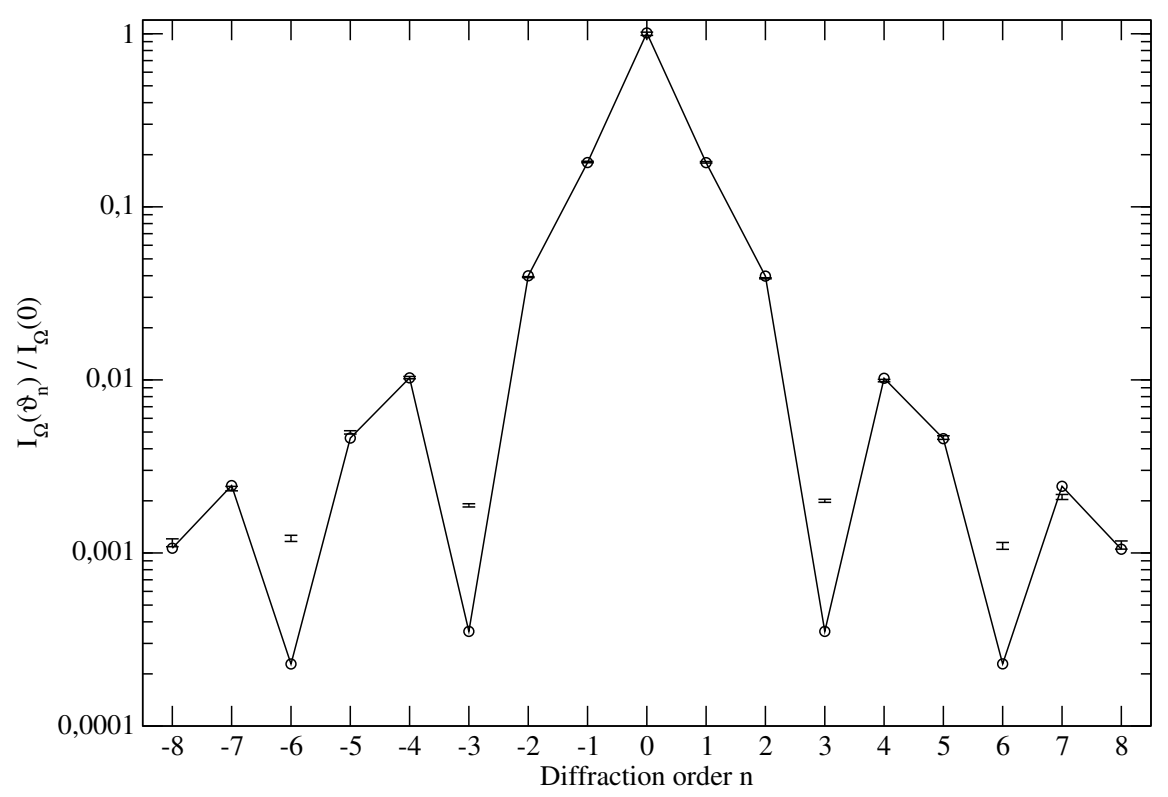

Figure 5.14: According to the hypothesis of concave bar sides the measured diffraction intensities of ground-state He at $v=1769.9 \frac{\mathrm{m}}{\mathrm{s}}$ are compared with a theoretical pattern which is based on a local wedge angle $\beta^{\text {loc }}=15.7^{\circ}$. On assigning low weight factors to the \pm 3 rd and \pm 6 th diffraction orders and using the theoretical value $C_{3}=0.136 \mathrm{meV} \mathrm{nm}^{3}$ for the dispersion interaction strength the geometrical slit width is fitted as $s=(67 \pm 0.2) \mathrm{nm}$ which is in agreement with the result of the $s_{\text {eff }}\left(\frac{1}{\sqrt{v}}\right)$ method. By comparison with Fig. 5.7 the full-weighted maxima are seen to be much better reproduced in the present fit.

Fig. 5.14 shows that by leaving out the third and sixth order the other diffraction order intensities are clearly better reproduced than in the comparable fit shown in Fig. 5.7. The new fit yields for the slit width $s=(67.0 \pm 0.2) \mathrm{nm}$ which is consistent with the result of the $s_{\text {eff }}\left(\frac{1}{\sqrt{v}}\right)$ method, while the other fit parameter $\sigma_{0}=1.7 \mathrm{~nm}$ is practically unchanged.

Finally, it remains to say that the above considerations are independent of retardation effects. This has been checked by fitting the cumulant-based relative diffraction intensities Eq. (5.11) to the theoretical diffraction intensities given in table 4.1. For the non-retarded case one obtains $s_{\text {eff }}=(65.3 \pm 0.15) \mathrm{nm}$, $\delta=(1.3 \pm 0.1) \mathrm{nm}$, and $\sigma=(0.6 \pm 0.1) \mathrm{nm}$, which is practically identical to the retarded case which yields $s_{\text {eff }}=(65.4 \pm 0.15) \mathrm{nm}, \delta=(1.2 \pm 0.1) \mathrm{nm}$, and $\sigma=(0.6 \pm 0.1) \mathrm{nm}$. From this example it can be concluded that retardation effects have no significant influence on the effective slit width $s_{\text {eff }}$. 


\subsection{Repulsive Potential}

A closer look at the figures Fig. 5.11 and Fig. 5.13 reveals that the curves of the experimentally determined curves of the effective slit widths $s_{\text {eff }}\left(\frac{1}{\sqrt{v}}\right)$ for different atomic species and different gratings bear a certain resemblance in that the data points seem to oscillate about the straight line that has been fitted to them according to Eq. (5.17). It will be shown in the following that it is theoretically expected to observe some kind of oscillation in the $s_{\text {eff }}\left(\frac{1}{\sqrt{v}}\right)$ curves. The origin of the new feature lies in the repulsive short-range part of the atom-bar potential, in particular, the depth $D$ of the potential well near the surface.

In order to understand the cause of the oscillations of $s_{\text {eff }}\left(\frac{1}{\sqrt{v}}\right)$ it is sufficient to look at the simplified expression Eq. (5.24) for $s_{\text {eff }}$ which is re-stated here for clarity:

$$
s_{\text {eff }}=2 \int_{0}^{\frac{s}{2}} \mathrm{~d} \zeta \cos \left(\frac{A}{\zeta^{2}}\right),
$$

with $A \equiv \frac{C_{3}}{2 \hbar v \tan \beta}$. Recalling the results of the eikonal approximation in chapter 2 one notes that the divergence of the argument of the cosine in Eq. (5.31) for the limit $\zeta \rightarrow 0$ is a direct consequence of an analogous behaviour of the dispersion potential $V(l)=\frac{-C_{3}}{l^{3}}$ when the atom-surface distance $l$ tends to zero. Including the atom-surface repulsion one has

$$
V(l)=\frac{-C_{3}}{l^{3}}+\frac{C_{9}}{l^{9}}
$$

which in the eikonal approximation and including the grating geometry leads to

$$
s_{\mathrm{eff}}=2 \int_{0}^{\frac{s}{2}} \mathrm{~d} \zeta \cos \left(\frac{A}{\zeta^{2}}-\frac{B}{\zeta^{8}}\right)
$$

which is just the generalization of Eq. (5.31), and where $B:=\frac{C_{9}}{8 \hbar v \tan \beta}$ has been defined. Fig. 5.15 shows a plot of the argument

$$
\varphi(\zeta):=\frac{A}{\zeta^{2}}-\frac{B}{\zeta^{8}}
$$

of the cosine in Eq. (5.33). A simple way to include the contribution arising from the repulsive part of the potential is to approximately set

$$
\tilde{\varphi}(\zeta) \approx\left\{\begin{array}{c}
\frac{A}{\zeta^{2}}: \quad \zeta>\zeta_{0} \\
\infty \quad: \quad \zeta \leq \zeta_{0}
\end{array}\right.
$$




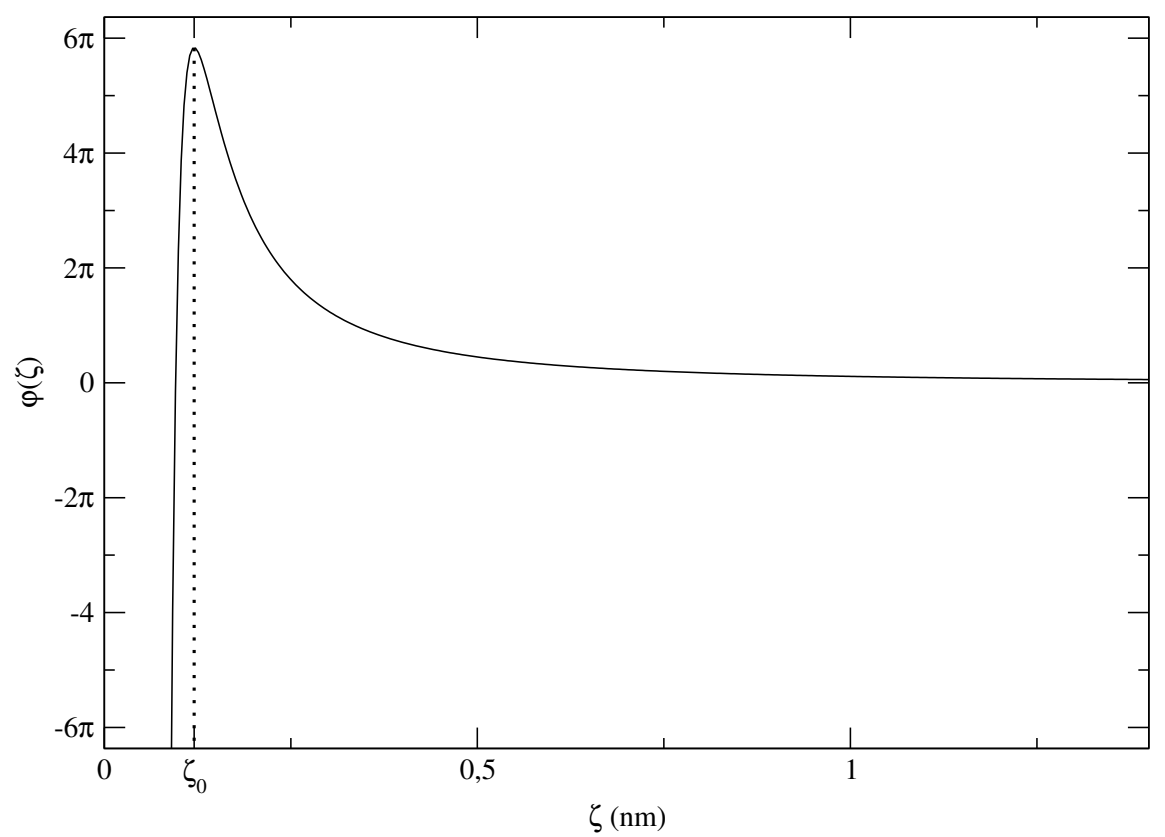

Figure 5.15: The quantum mechanical phase $\varphi(\zeta)$ for ground-state He atoms and a silicon nitride grating bar runs through a maximum at $\zeta=\zeta_{0}=0.12 \mathrm{~nm}$ near the bar surface at $\zeta=0$ if the repulsive part of the atom-bar potential is taken into account.

where

$$
\zeta_{0}=\left(\frac{C_{9}}{C_{3}}\right)^{\frac{1}{6}}
$$

marks the maximum of the function $\varphi(\zeta)$. Hereby it is worth noting that $\zeta_{0}$ does not depend on the velocity $v$. The approximation Eq. (5.35) is also indicated in Fig. 5.15. As a consequence of the approximation, Eq. (5.33) can be written as

$$
s_{\text {eff }}=2 \int_{\zeta_{0}}^{\frac{s}{2}} \mathrm{~d} \zeta \cos \left(\frac{A}{\zeta^{2}}\right),
$$

where the lower boundary of the integral has been replaced by the cut-off $\zeta_{0}$ which takes approximate account of the repulsive part of the atom-bar potential. Applying the same substitution that has led to Eq. (5.25) one finds

$$
s_{\mathrm{eff}}=\sqrt{A}\left\{\left[\frac{-2 \cos \xi}{\sqrt{\xi}}\right]_{\frac{4 A}{s^{2}}}^{\frac{A}{\zeta_{0}^{2}}}-2 \int_{\frac{4 A}{s^{2}}}^{\frac{A}{\zeta_{0}^{2}}} \mathrm{~d} \xi \frac{\sin \xi}{\sqrt{\xi}}\right\} .
$$

For ground-state He atoms and the $\mathrm{SiN}_{x}$ grating bars the cut-off comes out as $\zeta_{0}=0.12 \mathrm{~nm}$, while $(0.5 \mathrm{~nm})^{2} \leq A \equiv \frac{C_{3}}{2 \hbar v \tan \beta} \leq(1.0 \mathrm{~nm})^{2}$ (note the $v$ dependence 
of $A$ ). In the limit $\zeta_{0} \rightarrow \infty$ the upper boundaries in Eq. (5.38) tend to infinity and the previous result Eq. (5.17) is obtained, whereas for the given values of $\zeta_{0}$ and $A$ the upper boundaries are $17 \leq \frac{A}{\zeta_{0}^{2}} \leq 70$. The reason for the oscillations in the $s_{\text {eff }}$ curves becomes apparent after a look at the plot Fig. 5.16 of the integrand in Eq. (5.38). Apparently, functions of the type of $\frac{\sin \xi}{\sqrt{\xi}}$ converge slowly so that from

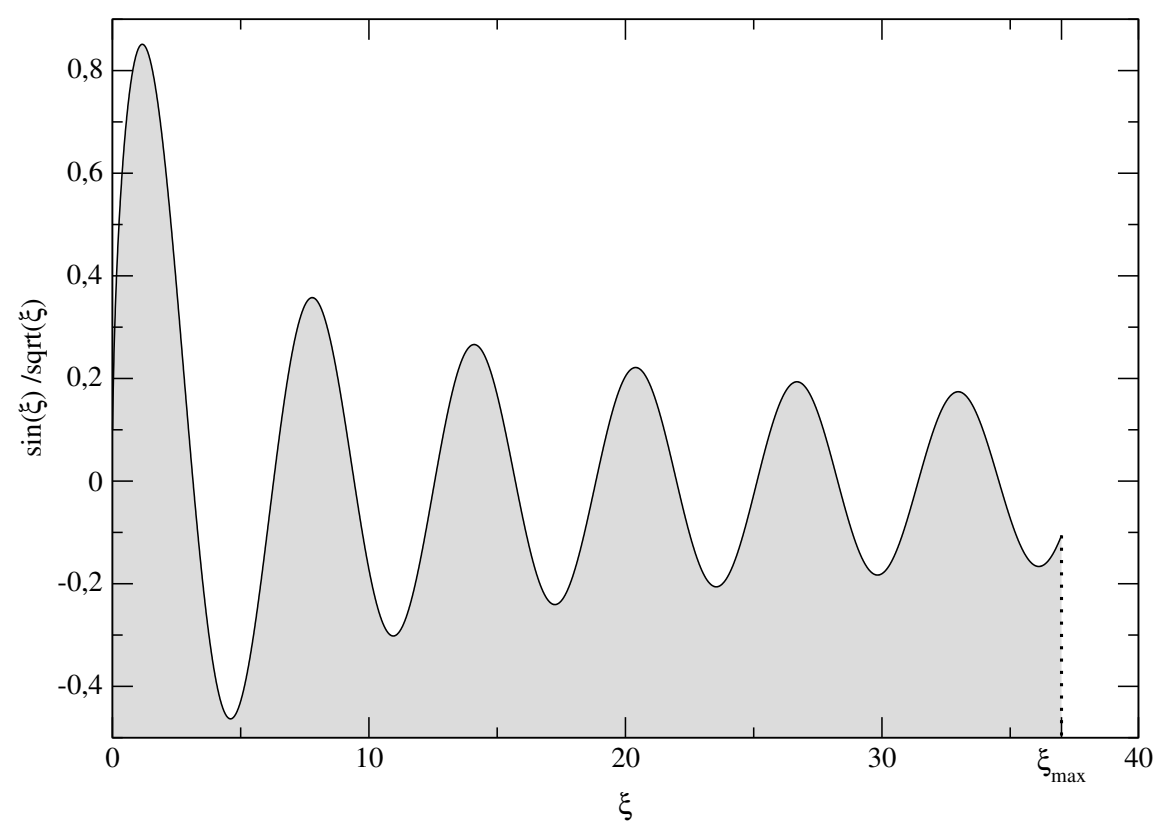

Figure 5.16: For large and small velocities $v, \xi_{\max }$ varies between 17 and 70 , so that the integral (shaded area) as a function of the velocity $v$ displays an oscillatory behaviour.

Eq. (5.38) an oscillatory behaviour of $s_{\text {eff }}$ is expected as the upper boundary $\frac{A}{\zeta_{0}^{2}} \equiv$ $\frac{C_{3}}{2 \hbar v \tan \beta \zeta_{0}^{2}}$ of the integral varies with the beam velocity $v$. On further evaluation, Eq. (5.38) reads

$$
s_{\text {eff }}=s-2 \zeta_{0} \cos \left(\frac{4 A}{\zeta_{0}^{2}}\right)-2 \sqrt{A} \int_{\frac{4 A}{s^{2}}}^{\frac{A}{\zeta_{0}^{2}}} \mathrm{~d} \xi \frac{\sin \xi}{\sqrt{\xi}},
$$

which shows that another oscillating cosine term appears in the expression. In view of the coarse approximation that has been made for the repulsive part of the potential in Eq. (5.35) the amplitude of the oscillations, which is of the order of a few Angstrøm, marks an upper limit to the oscillations observed with the exact potential. 
In an exact treatment, the effective slit width $s_{\text {eff }}$ is given by

$$
s_{\text {eff }}=\operatorname{Re} \int_{0}^{s} \mathrm{~d} x \mathrm{e}^{\mathrm{i} \varphi(x)} \equiv \operatorname{Re} f_{\text {slit }}\left(\vartheta_{0}\right),
$$

where the quantum mechanical phase $\varphi(x)$ that includes the repulsion is given by equation Eq. (4.14). The origin of the oscillations of $s_{\text {eff }}$ as a function of the velocity $v$ can be visualized by plotting the integral in Eq. (5.40) as a vectorial sum of complex numbers $\mathrm{d} x \mathrm{e}^{\mathrm{i} \varphi(x)}$ in the complex plane. The effective slit width is then the difference between the real part of the starting point and the end point of the resulting track, while the quantity $\delta$ corresponds to the imaginary part of the difference between these points. Furthermore it can be seen from Eq. (5.14) that the zeroth order intensity is given by the absolute value of the distance between the start and the end of the curve in the complex plane. Fig. 5.17 shows such a plot for ground-state He atoms at $v=1500 \frac{\mathrm{m}}{\mathrm{s}}$ diffracted from grating 5-3-1. The figure shows that the atom-bar interaction at both ends of the slit (both ends of the curve) bends the path in the Gaussian plane so that with increasing potential near the bar surface the track rolls up in a spiral whose sense of rotation is determined by the sign of the force the atom experiences at a certain distance from the bar surface. Without repulsion each end point of the curve converges in the centre of a single spiral that is produced by the attractive dispersion potential. With repulsion, the process of convergence is interrupted when the repulsion starts dominating the attraction for distances close to the bar. This changes the sense of rotation of the spiral so that the end pieces of the track move out of the centre region of their respective vertices before they wind up themselves as a result of the increasing repulsion at the bar surface. The actual convergence is thus achieved in points off-centre with respect to the convergence spirals of the attractive interaction alone.

By increasing the particle velocity $v$ one reduces the curvature of the whole track because in Eq. (5.40) the phase $\varphi(x)$ is proportional to $\frac{1}{v}$ (cf. Eq. (4.14)). This becomes manifest in the curves shown in Fig. 5.17 in that the spirals unwind. During this process the mentioned appendices which contain the important end points of the track rotate about the centre of the spirals to which they are attached. This leads to an oscillation of the distance between both end points that is observed in the experiment and becomes apparent in the oscillations of $s_{\text {eff }}\left(\frac{1}{\sqrt{v}}\right)$ in Fig. 5.13. The rotation of the end pieces of the convergence spirals upon variation of the beam velocity $v$ is illustrated in Fig. 5.18.

From the figure it can be seen that the value of the oscillating part of $s_{\text {eff }}(v)$ runs through a local maximum if the end point of the track lies to the right of the centre of the larger convergence spiral, because then the additional length of the appendix is counted as real and so increases the effective slit width $s_{\text {eff }}$. One may approximately characterize the situation by the complex differential increment 


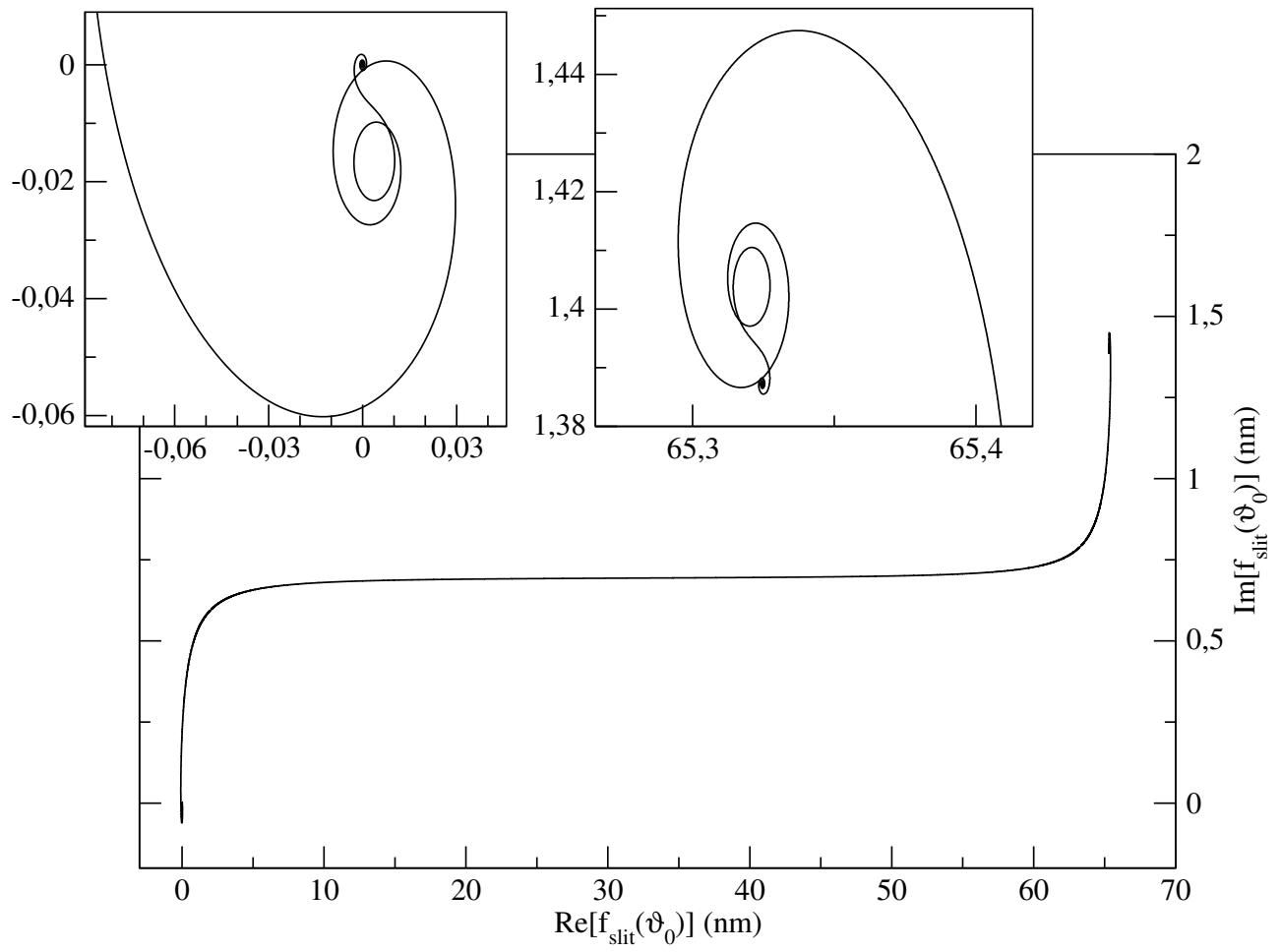

Figure 5.17: The integral Eq. (5.40) which is equal to the slit amplitude $f_{\text {slit }}\left(\vartheta_{0}\right)$ is visualized in the complex plane as a vector sum of complex differential increments $\mathrm{d} x \mathrm{e}^{\mathrm{i} \varphi(x)}$. The complex value of the slit amplitude can be read from the plot as the end point of the track which starts at the origin. The curvature depends in sign and magnitude on the value of the phase $\varphi(x)$ which in turn depends on the atom-bar potential. In the two in-sets the end regions of the track are shown where the potential minimum near the bar surface is reflected in a change of curvature so that the track does not wind up in a single spiral as would be the case for a solely attractive atom-bar potential but rather evades the centre of the spiral and forms a little appendix where it forms a quickly rotating vortex up as a result of the strongly increasing repulsion of the atoms near the bar surface.

$\mathrm{d} \zeta \mathrm{e}^{\mathrm{i} \varphi\left(\zeta_{0}\right)}$ being real and positive at the turning point of the track $\mathrm{\rrbracket}$. This means that the phase $\varphi\left(\zeta_{0}\right)$ (cf. Fig. 5.15) is an integer multiple of $2 \pi$. With the help of Eq. (5.36) for $\zeta_{0}$, Eq. (5.34) yields in this case

$$
\varphi\left(\zeta_{0}\right)=n 2 \pi=\frac{3 C_{3}^{4 / 3}}{8 \hbar v_{n} \tan \beta C_{9}^{1 / 3}}=K^{2} \frac{3 C_{3}^{1 / 3}}{8 \pi v_{n} C_{9}^{1 / 3}},
$$

where $K$ is the slope of $s_{\text {eff }}\left(\frac{1}{\sqrt{v}}\right)$ as introduced in Eq. (5.18).

For very high velocities $v$ the tracks of the type shown in Fig. 5.18 of Fig. 5.17

\footnotetext{
${ }^{1}$ i.e. where the curvature changes sign
} 


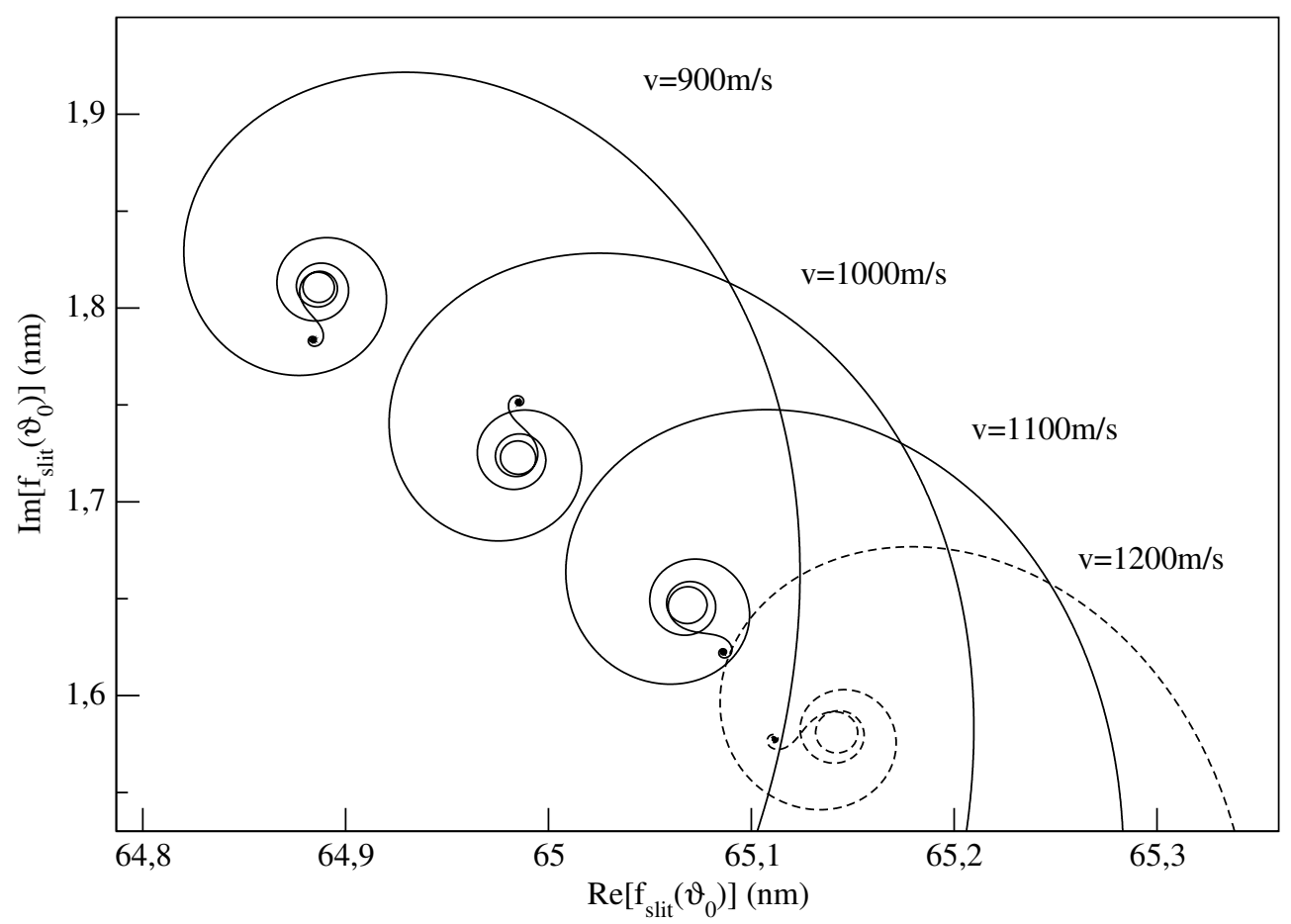

Figure 5.18: The real coordinate of the end points of each track is identical to the effective slit width $s_{\text {eff }}$ while the imaginary coordinate gives the quantity $\delta$. The centres of the larger spirals of each track represent the values of $s_{\text {eff }}$ and $\delta$ without the repulsive interaction. These centres wander along as $v$ is increased. With the repulsive interaction included the end points of the track are found at the end of little appendices which rotate about the centres as $v$ is increased. This rotation causes the oscillations of $\delta$ and $s_{\text {eff }}$ as functions of the beam velocity $v$.

are not curly but straight and real with a length $s_{\text {eff }}(v \rightarrow \infty)=s$, equal to the geometrical slit width. Decreasing the beam velocity the right half of the track bends upwards, and for the velocity

$$
v_{1}=K^{2} \frac{3 C_{3}^{1 / 3}}{16 \pi^{2} C_{9}^{1 / 3}}
$$

the track has completed its first revolution. According to Eq. (5.41) the subsequent revolutions of the track as a consequence of a decreasing beam velocity $v$ are completed at velocities

$$
v_{n}=\frac{v_{1}}{n} .
$$

For the oscillations of $s_{\text {eff }}\left(\frac{1}{\sqrt{v}}\right)$ in Fig. 5.13 this means that the maxima of the modulation are expected to appear at $\frac{1}{\sqrt{v_{1}}}, \sqrt{\frac{2}{v_{1}}}, \sqrt{\frac{3}{v_{1}}}$, etc. From the definition 
Eq. (5.32) of the Lennard-Jones potential $V(l)$ the well depth

$$
D=-\frac{2 \sqrt{3}}{9 \sqrt{C_{9}}}\left(C_{3}\right)^{3 / 2}
$$

can be derived, which is equivalent to

$$
\left(\frac{-3 \sqrt{3} D}{2 C_{3}}\right)^{\frac{2}{3}}=\left(\frac{C_{3}}{C_{9}}\right)^{\frac{1}{3}} .
$$

Inserting Eq. (5.45) into Eq. (5.42) one finds after a little algebra

$$
D \approx n^{\frac{3}{2}} 147 C_{3}\left(\frac{v_{n}}{K^{2}}\right)^{\frac{3}{2}}
$$

which makes it possible to determine the well depth $D$ from the maxima of the modulation of $s_{\text {eff }}\left(\frac{1}{\sqrt{v}}\right)$.

In order to illustrate the concepts just explained and despite the few number of available data points it is tried to fit theoretical $s_{\text {eff }}\left(\frac{1}{\sqrt{v}}\right)$ curves that include the repulsive part of the atom-bar potential to the measured curves of He and $\mathrm{D}_{2}$ with grating 5-3-1 and 5-2-2 as presented earlier. The results are shown in Fig.5.19 and Fig.5.20. The potential well depths $D$ that have been employed in the fit are for $\mathrm{D}_{2} D=-50.8 \mathrm{meV}$ and for the two samples of $\mathrm{He} D=-34.6 \mathrm{meV}$ which are reasonable values in view of the known well depths of these particles interacting with other insulator surfaces. For the special amorphous silicon nitride material of the gratings, no comparable values are available in the literature. It is somewhat reassuring to see that for the two samples of He diffracted from the gratings 5-2-2 and 5-3-1 the same atom-surface parameter set $C_{3}, C_{9}$ is able to mimic the local trends in the respective $s_{\text {eff }}\left(\frac{1}{\sqrt{v}}\right)$ curves.

The fact that the effective slit width can reveal details of the atom-bar potential concerning the repulsive part adds a new quality to the ongoing diffraction experiments. Unfortunately, the experimental accuracy has so far not been aimed at measuring the effect, especially the limited number of data points in the measured $s_{\text {eff }}\left(\frac{1}{\sqrt{v}}\right)$ curves makes it difficult to infer any structure more complicated than a straight line. An improvement on this issue can obviously be achieved by providing more data points.

A possibly more distinct evidence of the same effect could be achieved by measuring the absolute intensity $I_{\Omega}\left(\vartheta_{0}\right)$ of the zeroth diffraction order at different beam velocities $v$. According to Eq. (5.14) one has

$$
I_{\Omega}\left(\vartheta_{0}\right) \propto s_{\mathrm{eff}}^{2}(v)+\delta^{2}(v) .
$$

In Fig. 5.17 this quantity is equal to the squared absolute distance between the end points of the shown curve. The rotation of each end point with varying velocity 


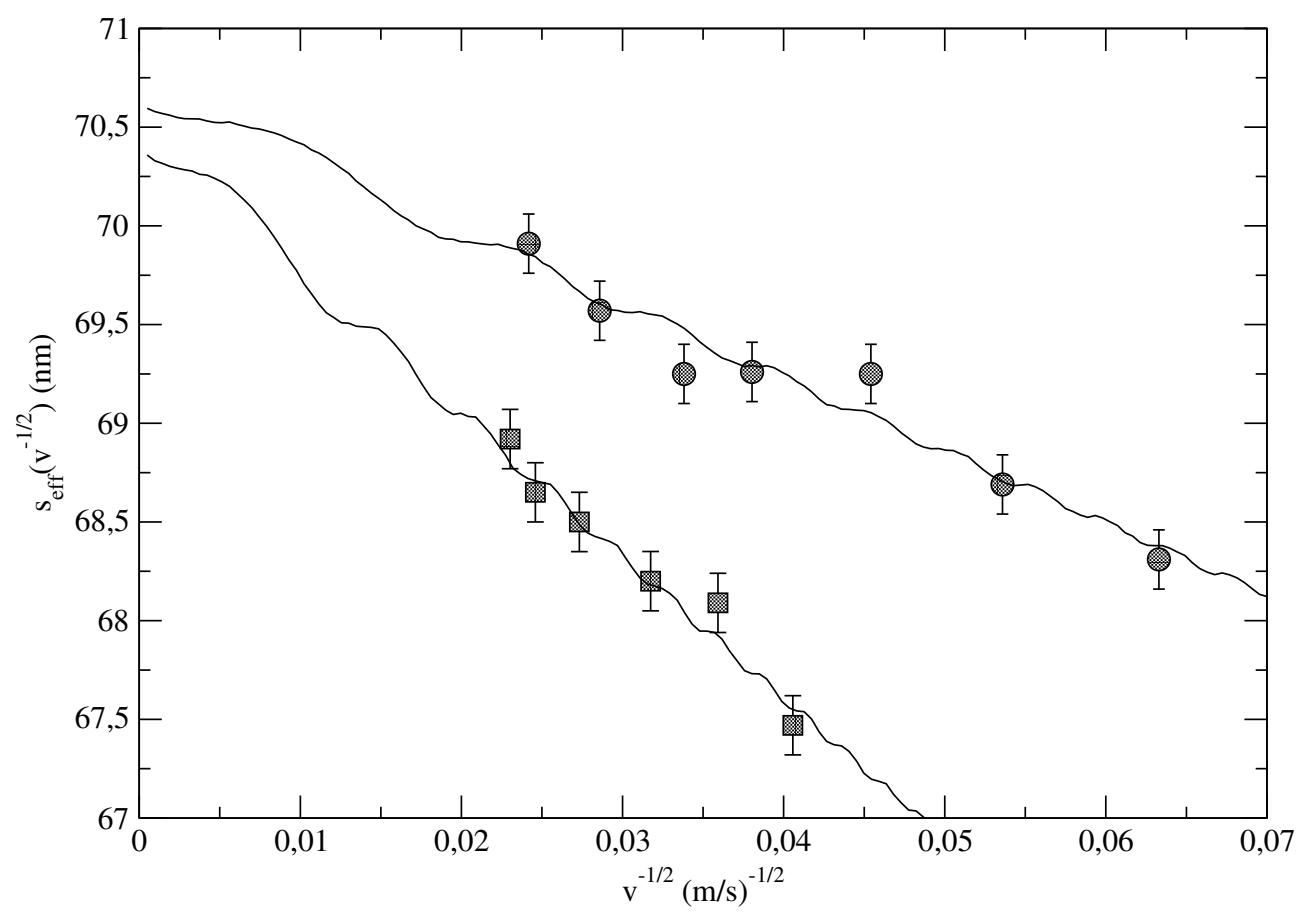

Figure 5.19: The data points of the effective slit width $s_{\text {eff }}\left(\frac{1}{\sqrt{v}}\right)$ for ground-state He (circles) and $\mathrm{D}_{2}$ (squares) are compared with theoretical curves that take account of the atom-surface potential well. Adjusting the theoretical curves by hand with the help of the repulsive potential strength $C_{9}$ reasonable agreement with the experiment is achieved for values of $C_{9}$ that correspond via Eq. (5.44) with potential well depths $D=-34.6 \mathrm{eV}(\mathrm{He})$ and $D=-50.8 \mathrm{eV}\left(\mathrm{D}_{2}\right)$.

$v$ as described above and illustrated in Fig. 5.18 clearly leads to an oscillatory behaviour of $I_{\Omega}\left(\vartheta_{0}\right)$. The magnitude of the expected oscillations can be estimated from Fig. 5.21 where $I_{\Omega}\left(\vartheta_{0}\right)$ is plotted as a function of the beam velocity $v$, for a grating like 5-3-1 and an atom-surface potential $V(l)$ like in Eq. (5.32).

It may be pointed out that the presence of steps in Fig. 5.21 is a distinct feature of matter diffraction which is absent in the diffraction of light of a comparable wavelength. The steps reflect a quantisation of the transmission through the grating as a result of Planck's constant appearing in the denominator of the phase $\varphi(x)$ in Eq. (4.14) that arises from the atom-bar potential.

Another way to observe this quantised transmission is to repeat the measurement of the zeroth order intensity $I_{\Omega}(\tau)$ under grating rotations $\tau$ at a higher precision than has been sufficient so far. In this type of experiment the change in the phase $\varphi(x, \tau)$ is not accomplished by changing the beam velocity $v$ but rather through the grating rotation $\tau$ by which the atomic beam passes the grating bars at different angles on each side of the slit. The theory presented in chapter 4 is 


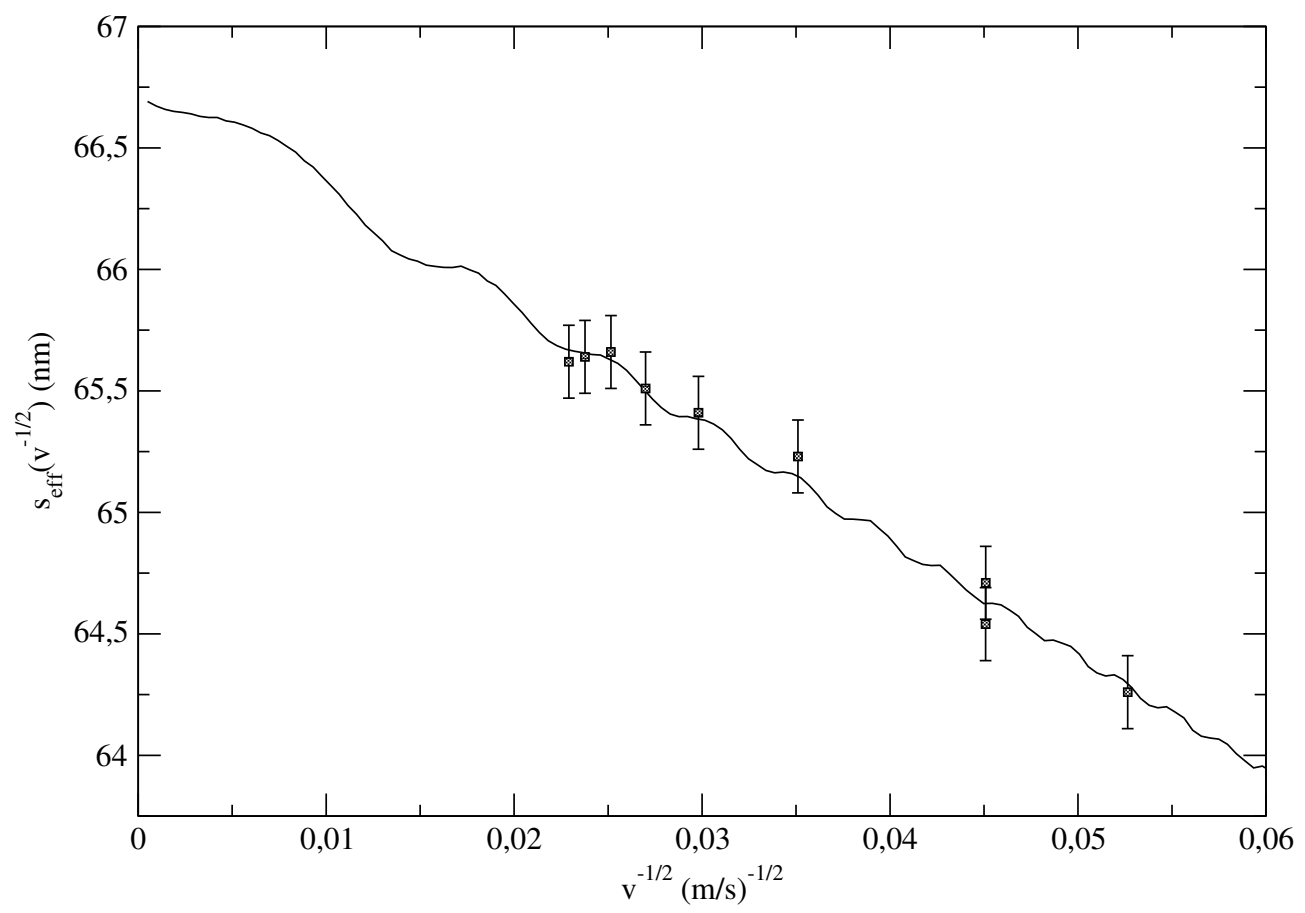

Figure 5.20: The atom-surface potential well depth $D=-34.6 \mathrm{eV}$ as fitted to data (cf. Fig. 5.19) for ground-state He atoms diffracted from grating 5-2-2 reproduces reasonably well also the data of $s_{\text {eff }}\left(\frac{1}{\sqrt{v}}\right)$ obtained for the grating 5-3-1.

straightforward in predicting a transmission curve with the repulsive potential included. Fig. 5.22 shows a comparison of an experimental transmission curve of ground-state He atoms and grating 5-3-1 with a theoretical curve. It is not possible to speak of any discernible structure in the experimental curve that could be compared to the theory. The noise that is visible in the random fluctuations of the experimental curve can be traced back to mainly mechanical reasons inside the apparatus so that it appears feasible to achieve higher precision in this type of experiment, which would allow to to check for the predicted oscillatory behaviour.

Concluding the issue, it is stressed again that the oscillations are a consequence of the fact that there is potential well at the bar surface, regardless of its specific form. In particular, the oscillations are expected to be observable despite the corrugation of the bar surface. In lowest order, as presented earlier, this is true because the assumption of locally straight bar edges renders the bar surface potential equal to that used in the above predictions 2 . In a detailed treatment,

\footnotetext{
${ }^{2}$ In Fig. 5.15, p.109 the corrugation does not shift the maximum of the phase $\varphi(\zeta)$ because $\zeta$ is defined as the distance from the corrugated edge and hence follows the undulation as different vertical positions are considered
} 


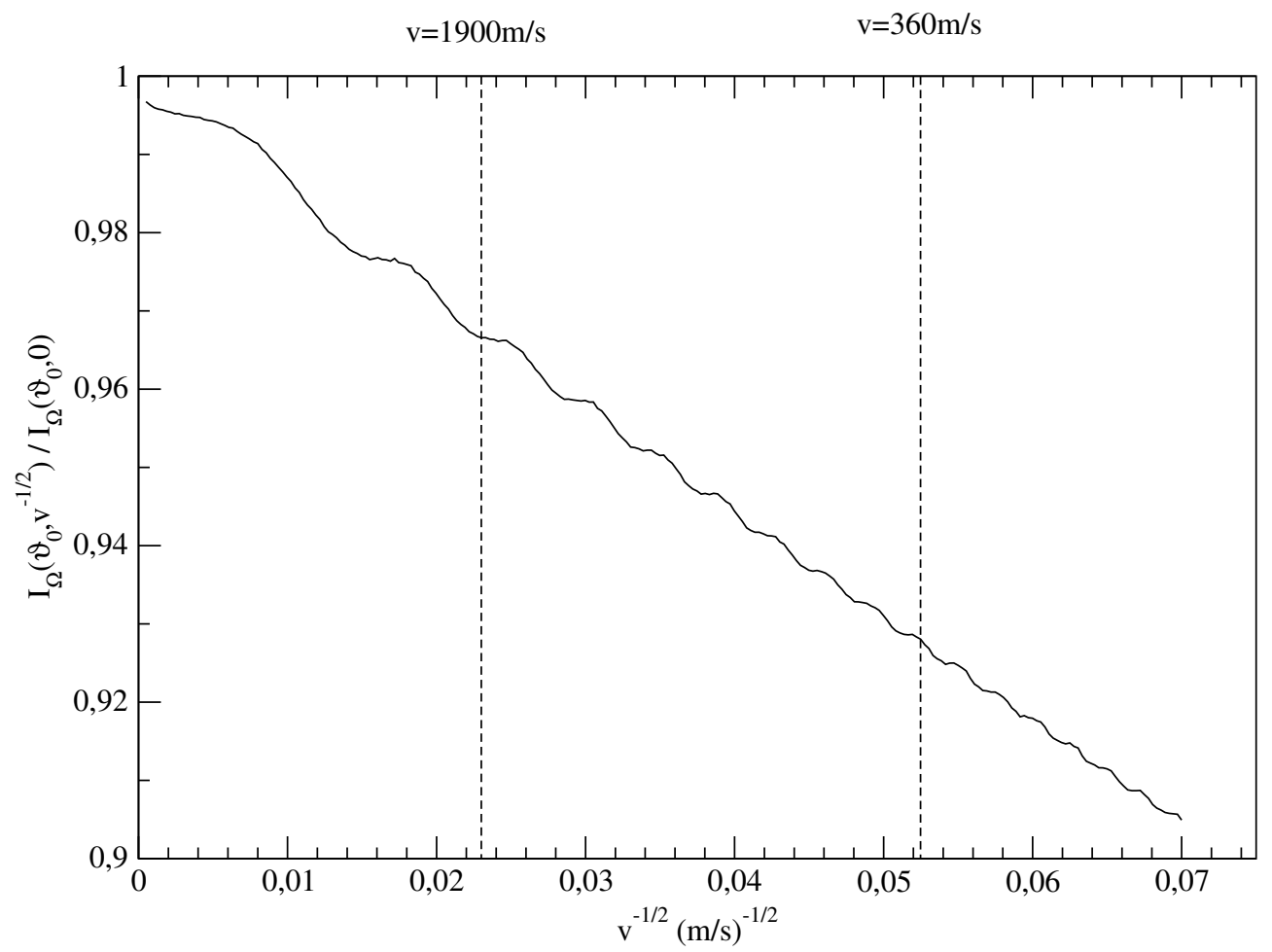

Figure 5.21: The dashed lines mark the experimentally accessible velocity range for ground-state He atoms. Within this range, the theory predicts clearly discernible steps in the absolute intensity of the the zeroth order diffraction maximum.

the effective surface potential and hence the relevant quantum mechanical phase $\varphi(x)$ is modified by the corrugation, for example through terms with a different power law with respect to the impact parameter. However, this does not alter the central feature that the phase runs through a global maximum near the surface which leads to the oscillations.

\subsection{Error Analysis}

As the reader is now familiar with most intricacies of the atom diffraction technique it is considered the best opportunity to embark on a little digression concerned with the origin of the frequently mentioned difference between the real and the theoretically assumed form of the interaction potential of the atoms and the grating bars. It is illustrated in the figures Fig. 5.7 and Fig. 5.14 that the measured diffraction intensities for ground-state He atoms exhibit some systematic deviation from the theoretical predictions, especially for the 3rd and 6th diffraction orders. The same discrepancies also appear for other ground-state species 


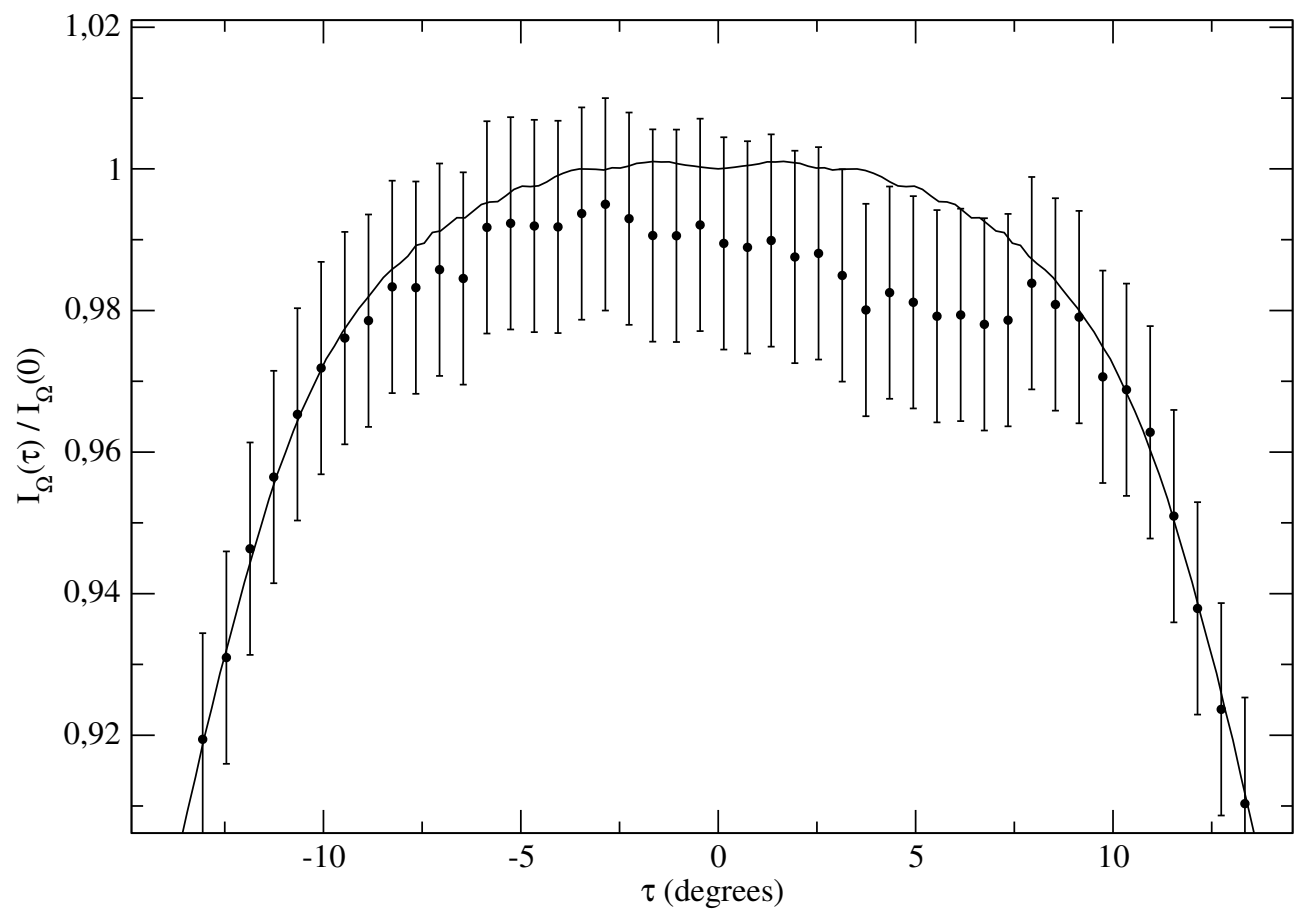

Figure 5.22: The measured zeroth order intensity of ground-state He atoms at $v=$ $1769.9 \frac{\mathrm{m}}{\mathrm{s}}$ (full circles with error bars) as a function of the rotation angle $\tau$ of grating 5-31 is compared with a theoretical curve that takes account of the atom-surface potential well. Despite some promising features in the experimental data there is no significant agreement between the theoretically predicted oscillations for small rotation angles and the experimental data which suffer most from errors in this region of interest.

such as those measured by Grisenti et al. [12], and they cannot be removed consistently for all beam velocities $v$ by variation of the parameters $t, d, s$, and $\beta$ that describe the grating geometry, nor by using different values for the dispersion interaction strength $C_{3}$ or the damping parameter $\sigma_{0}$. As mentioned earlier, the possibility of experimental background noise has been ruled out recently when the diffraction experiments with ground-state He were repeated after a careful over-all check of the apparatus. In particular, the grating orientation has been accurately fixed to the standard position of the grating normal being parallel to the incident beam, and the bars at right angles to the scattering plane. A number of other issues, e.g. the purity of the incident beam have also been settled. They are dealt with later on in connection with the diffraction of metastable atoms.

From the above considerations it is concluded that the origin of the discrepancy must lie beyond the theoretical description originally available. In sum, this description is based on strictly two-dimensional scattering theory with ideally trapezoid-shaped grating bar cross-sections and straight vertical bar edges. The atom-bar interaction is initially assumed to consist of an infinitely repulsive po- 
tential and an attractive term that rests on the non-retarded limit of the dipole approximation of the dispersion potential between the atom and the material of the grating bars. As the need now arises, the given theoretical concept leaves room for many hypotheses and possible improvements in order to explain the deviant features.

As a first possibility, retardation effects have been shown in section 4.4 to be too small to explain the deviations which amount to up to $50 \%$ in the third and sixth order diffraction intensities, moreover the theoretical 3rd and 6th order intensities tend to become smaller with retardation included which adds to the difference between theory and experiment.

Second, the presence of some repulsive potential near the surface has been shown to lead to small oscillations of the diffracted intensities as a function of the beam velocity $v$ but the amplitude of the effect is generally too small to account for the observed discrepancies.

Third, the possibility of significant terms with other power laws being present in the atom-surface potential, describing higher multipoles of the dispersion interaction, or arising from the surface corrugation, cannot account for the discrepancies. In chapter 4 it is shown that the quantum mechanical phase $\varphi(x)$ can be easily calculated for those additional potentials of the form

$$
V_{n}(l)=\frac{ \pm C_{n}}{l^{n}} \quad, \quad n \in\{2,3,4, \ldots\} .
$$

On comparing the resulting theoretical diffraction intensities and the theoretical $s_{\text {eff }}\left(\frac{1}{\sqrt{v}}\right)$ curves it becomes apparent that in order to significantly alter the theoretical 3rd and 6th order intensities the additional potentials have to be very strong which leads to a clear deviation of the $s_{\text {eff }}\left(\frac{1}{\sqrt{v}}\right)$ curve from a straight line which is not observed in reality. In other words, the measured $s_{\text {eff }}\left(\frac{1}{\sqrt{v}}\right)$ curve rules out the possibility of significantly strong additional potentials, hence they cannot be the origin of the observed discrepancies between theoretical and experimental diffraction intensities. The same is true regarding the repulsive contribution to the atom-surface potential which can be modelled by an additional $\frac{C_{9}}{l^{9}}$ term in the potential. The adaption of this term to the idealized grating bar geometry has been demonstrated in section 4.1.

In section 4.5 the theoretical treatment of adsorbate layers on the grating bars has revealed that such layers can indeed produce modifications in the theoretical diffraction intensities that resemble the measured features. However, in recent experiments the transmission gratings are heated up to a temperature of a few hundred degrees Celsius which is known to evaporate any possible adsorbate layer, and the discrepancies in the intensity pattern are seen to persist [102]. This allows to conclude that the discrepancies are not caused by adsorbate layers on the grating bars. 
The influence of the bar surface corrugation is shown in section 4.3 to lead to a mere damping of the diffracted intensities which cannot explain the deviations of the 3rd and 6th order intensities. In higher order, the corrugation can lead to an effective change of the atom-bar potential, which in principal may affect the relative intensities. The corrections to the potential can always be expressed within an expansion of the total potential in terms of a sum of inverse power laws like Eq. (5.48). It has been demonstrated above that any significant contribution from terms of a power law different from the usual dispersion potential $V_{3}(l)$ leads to a change in the effective slit width $s_{\text {eff }}\left(\frac{1}{\sqrt{v}}\right)$ which is not observed in the experiment. Therefore the corrugation of the bar surfaces cannot deliver the desired explanation of the discrepancies between theoretical and experimental diffraction intensities.

As the only plausible reason for the discrepancies remains that due to the manufacturing process the slit width $s$, as averaged over the width of the illuminated spot on the grating, and the vertical coherence length $L_{y}$, varies over the total height $Y \approx 5 \mathrm{~mm}$ of the illuminated spot. Generally, the vertical coherence length $L_{y}$ of the incident beam is much smaller than the total illuminated height $Y$, so that the detected intensity as a function of the scattering angle $\vartheta$ is always to be understood as an incoherent superposition of diffraction patterns generated at different vertical positions on the grating. In the case that the average slit width $s$ is not constant for different vertical positions on the grating the measured diffraction intensities are an incoherent superposition of different diffraction patterns, namely those arising from different average slit widths.

With the help of this work's repeatedly employed theoretical example of ground-state helium atoms at $v=1769.9 \frac{\mathrm{m}}{\mathrm{s}}$ diffracted from grating $5-3-1$ it is shown in Fig. 5.23 that an incoherent superposition of diffraction intensities arising from slit widths uniformly distributed between $s-4 \mathrm{~nm}$ and $s+4 \mathrm{~nm}$ is practically equal to a single pattern generated with the measured slit width $s=66.8 \mathrm{~nm}$, except that the 3rd and 6th order intensities of the former pattern are higher by a factor two. This is remarkable because from Fig. 5.14 it can be seen that the theoretical diffraction intensities based on the new concept of a local wedge angle $\beta^{\text {loc }}$ are in agreement with the measured intensities except for the 3rd and 6th order. With the assumption on the varying average slit width $s$ the theoretical and experimental patterns are in complete agreement for all beam velocities.

The Debye-Waller damping which is observed in the diffraction experiments with ground-state helium atoms is consistent with a variance $\sigma_{0}^{2}=(1.6 \mathrm{~nm})^{2}$ of the bar edges. From this the standard deviation $\Delta s$ of the slit width $s$ is obtained by [110:3]

$$
\Delta s=\sqrt{2 \sigma_{0}^{2}}=\sqrt{5.12} \mathrm{~nm} \approx 2.3 \mathrm{~nm} .
$$

The uniformly distributed slit widths used above have a standard deviation $\tilde{\Delta} s$ 


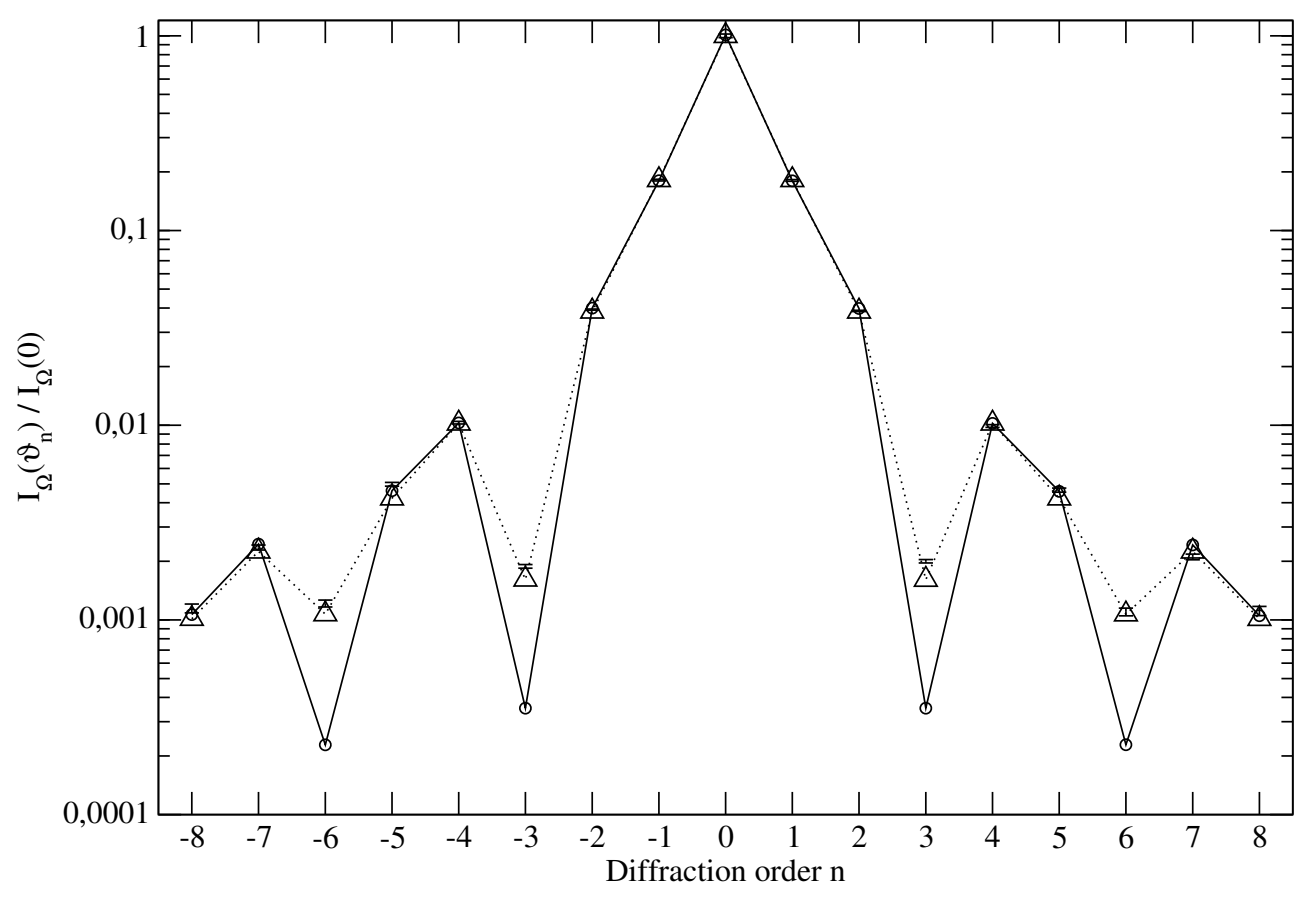

Figure 5.23: The experimental diffraction intensities (data points with error bars) are prefectly reproduced if the theoretical intensities are the result of an incoherent superposition of diffraction patterns arising from different slit widths $s$. In the figure, the example of ground-state He atoms is presented that is comparable to the figures Fig. 5.7 and Fig. 5.14. The best fit without any incoherent superposition (open circles connected with a solid line) fails to describe the \pm 3 rd and \pm 6 th order intensities. An incoherent superposition with slit widths uniformly distributed between $s-4 \mathrm{~nm}$ and $s+4 \mathrm{~nm}$ perfectly adjusts the \pm 3 rd and \pm 6 th order intensities while the other intensities remain unchanged.

of

$$
\tilde{\Delta} s=\left[\frac{1}{8} \int_{-4}^{4} \mathrm{~d} x x^{2}\right]^{1 / 2} \mathrm{~nm} \approx 2.3 \mathrm{~nm}
$$

which coincides with the measured value $\Delta s$. Note that $\Delta s$ and $\tilde{\Delta} s$ are supposed to be of entirely different origin. The first describes the bar edge corrugation and refers to a coherent effect, while the second suggests that the average bar width varies over the height of the grating.

It is concluded that the proposed concept is well suited to account for the measured 3rd and 6th order intensities. In reality, the average slit width $s$ is presumably rather normally than equally distributed along the height of the grating, but this is not expected to change the quality of the result. A good way to check the entire hypothesis is to diffract X-rays from the gratings used for the 
atom diffraction. Due to the variation of the average slit width the diffracted $\mathrm{X}$-ray intensities are expected to display the same deviations, namely their 3rd and 6th order intensities are to be measured twice as large as the Fraunhofer diffraction theory predicts.

\subsection{Diffraction Experiment with Metastable Atoms}

This subsection analyzes a series of diffraction experiments that have been performed in December 1999 by R. Grisenti at the Max-Planck-Institut für Strömungsforschung, Göttingen with metastable $\mathrm{He}^{*}$ and $\mathrm{Ne}^{*}$ atoms in order to determine the dispersion potential strength $C_{3}$ of both species with the silicon nitride material of the transmission grating 5-3-1 that was used throughout.

Experience has shown that the interpretation of the measured diffraction patterns in terms of the interaction constant $C_{3}$ is quite sensitive to a number of potential errors arising from the geometry and orientation of the transmission grating, and from the way the experiment is carried out. In order to achieve a relative error of at most $30 \%$ in $C_{3}$ it has proven inevitable to look at the sources of error in detail. The essential steps toward this goal have already been done in the preceding sections where the geometry of the transmission grating 5-3-1 has been found to be suitably characterized by the period $d=100 \mathrm{~nm}$, the slit width $s=(66.8 \pm 0.2) \mathrm{nm}$, and the total wedge angle $\beta=(11 \pm 0.5)^{\circ}$ of the grating bars. The bars are assumed to be ideal trapezoidal prisms because from the strong dispersion interaction of the metastable atoms it can be expected that the local inclination of the bar sides will be effectively averaged over the whole depth $t=57 \mathrm{~nm}$ to give the total wedge angle $\beta$. Using this assumption one avoids the arbitrariness of estimating the effective local wedge angle as specified for both diffracted atomic species at the price of an error in $C_{3}$ of about $10 \%$.

The remaining piece of investigation into the potential errors is concerned with the experimental apparatus a schematic yet detailed drawing of which is shown in Fig. 5.1. The following description of the experiment focuses on those aspects that are relevant for a general understanding and, in particular, for the discussion of the potential errors. A comprehensive account of the experimental details has been published in [54].

\subsubsection{Beam}

From a nozzle in the wall of a continously refilled high pressure chamber located at $z=-115 \mathrm{~cm}$ a supersonic jet of either He or Ne gas streams out into the vacuum inside the apparatus. The beam is directed along the $z$ axis towards the transmission grating 5-3-1 at the origin of the chosen coordinate frame. The 
grating bars are vertically oriented in $y$-direction while the $x$-axis is taken to point to the right when seen from the position of the beam source. Between an electrode inside the nozzle and a metal funnel ("skimmer"), whose narrow end at $z=-112 \mathrm{~cm}$ points toward the nozzle, a high-voltage discharge spark is maintained during the operation of the source. The discharge raises the atoms in the beam to all sorts of excited states which relax to the ground-state before the atoms reach the grating except for the respective metastable states of the $\mathrm{He}$ and Ne atoms whose life-times are known to be at least 10 times longer than it takes for the atoms to reach the detector at $z=730 \mathrm{~mm}$. From the $10^{11}$ atoms that arrive at the grating per second an average of only $10^{6}$ atoms are in metastable states, a disadvantage which is compensated for by the very high detection efficiency of the channel plate detector. After leaving the turbulent zone at the skimmer the atoms essentially propagate without mutual collisions in the beam whose atomic number density is about $10^{12} / \mathrm{m}^{3}$, corresponding to an average mutual distance of the atoms of about $0.1 \mathrm{~mm}$. The natural velocity distribution of the source of metastable atoms can be characterised by a Gaussian distribution around the mean velocity $v_{0}$ whose measured variance $\sigma_{v}^{2}$ in the case of $\mathrm{He}^{*}$ is $\frac{\sigma_{v}}{v}=13.3 \%$.

It is known from other experiments [133, 114] with beam sources of the given type that $98 \%$ of the metastable $\mathrm{He}^{*}$ atoms are in the ${ }^{3} \mathrm{~S}_{1}$ state and $2 \%$ are in the ${ }^{1} \mathrm{~S}_{0}$ state. Of the metastable $\mathrm{Ne}^{*}$ atoms, $85 \%$ are in the ${ }^{3} \mathrm{P}_{2}$ state and $15 \%$ are in the ${ }^{3} \mathrm{P}_{0}$ state. The difference in the respective states of $\mathrm{He}^{*}$ and $\mathrm{Ne}^{*}$ arises solely from the spin degree of freedom. All mentioned states can be imagined as one electron of the outer shell being raised to the next higher level which is an $s$ level for He atoms, and a $p$ level for Ne. The theoretical analysis in this text focuses on the respective major portions of the $\mathrm{He}^{*}$ and $\mathrm{Ne}^{*}$ beams, which is expected to lead to a small error in the case of $\mathrm{He}^{*}$ because the relative amount of atoms in the ${ }^{1} \mathrm{~S}_{0}$ state is small. In the case of $\mathrm{Ne}^{*}$, there are theoretical calculations on the dispersion interaction between atoms that predict a maximum of $10 \%$ changes in the interaction strength between various inter-combinations of the two metastable states [83]. From this it can be concluded that the interaction of the two metastable states of Ne with the grating bars will be equal within $10 \%$. The relative influence of the smaller portion in the $\mathrm{Ne}^{*}$ beam on the dispersion strength $C_{3}$ extracted from the measured diffraction patterns is thus expected to fall below $2 \%$, which is included in the error bars.

\subsubsection{Collimation and Diffraction}

After emerging from the skimmer at $z=-112 \mathrm{~cm}$ whose least diameter is $0.72 \mathrm{~mm}$ the cone-shaped atomic beam encounters a barrier at $z=-1 \mathrm{~m}$ with a rectangular collimation window whose centre falls on the $z$-axis, and which is $s_{1}:=20 \mu \mathrm{m}$ wide and $5 \mathrm{~mm}$ high. Each monochromatic ensemble of atoms in the beam undergoes 
quantum mechanical diffraction by this slit whereby the macroscopical dimension of the slit height leads to vertical diffraction effects as described in section 4.3. The subsequent passage of the atoms through one of the about $1 \mathrm{~mm}$-wide slots

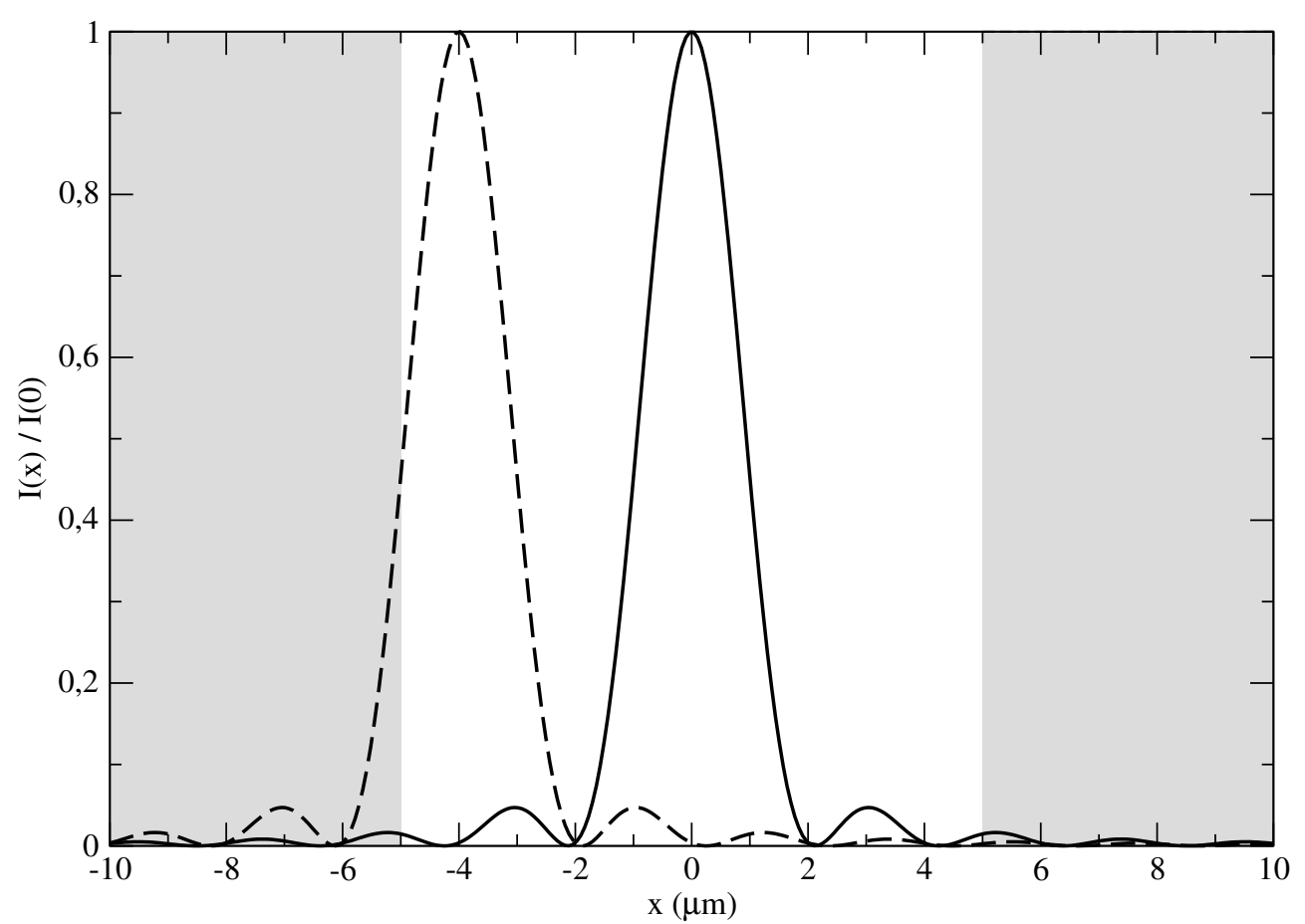

Figure 5.24: The figure shows the theoretical intensity of a ground-state He atom at $v=1769.9 \frac{\mathrm{m}}{\mathrm{s}}$ diffracted from the first collimator as evaluated in the plane of the second collimator which is placed $L=0.85 \mathrm{~m}$ downstream from the first and whose width is indicated by the shaded boundaries. For atoms that reach the first collimator at an incident angle $\vartheta_{i}=0$ the bulk of the intensity is seen to fall into the second collimation slit (solid line). The dashed curve is calculated for an atom that reaches the first collimator at an incident angle $\vartheta_{i}=4 \mu \mathrm{rad}$. As can be seen the left boundary of the second collimation slit clips the intensity profile. This effect determines the angular distribution of atoms impinging on the grating that lies behind the second collimation slit.

of the chopper at $z=-85 \mathrm{~cm}$ can be treated classically. On arrival at the second collimating slit at $z=-15 \mathrm{~cm}$ which is also $5 \mathrm{~mm}$ high and $s_{2}:=10 \mu \mathrm{m}$ wide each atom ${ }^{3}$ can be described by a quantum mechanical amplitude $A\left(x, \vartheta_{\mathrm{i}}\right)$ which in Fraunhofer diffraction theory is given by

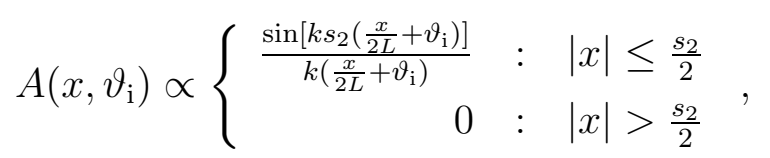

\footnotetext{
${ }^{3}$ Read, also in the following: Each monochromatic ensemble of atoms in the beam.
} 
where $\mathrm{L}=85 \mathrm{~cm}$ is the distance between the first and the second collimator and $\vartheta_{\mathrm{i}}$ stands for the angle at which the atom falls from the skimmer onto the first collimator.

It can be seen from Eq. (5.51) that this skimmer emission angle $\vartheta_{\mathrm{i}}$ leads to a sideway shift of the quantum mechanical amplitude $A\left(x, \vartheta_{\mathrm{i}}\right)$. For $\vartheta_{\mathrm{i}}=0$ the maximum of the amplitude falls on the centre of the second collimation slit whereas for $\left|\vartheta_{\mathrm{i}}\right|>\frac{s_{2}}{2 L}$ the maximum hits the edges of the second collimation slit and is partly cut off (cf. Fig. 5.24). From this one deduces that the number $N\left(\vartheta_{\mathrm{i}}\right)$ of atoms able to pass the second collimator and travel on to the grating is proportional to the probability of finding an atom emitted from the skimmer at an angle $\vartheta_{\mathrm{i}}$ inside the second collimation slit. This probability is given by

$$
\int_{-\frac{s_{2}}{2}}^{\frac{s_{2}}{2}} \mathrm{~d} x\left|A\left(x, \vartheta_{\mathrm{i}}\right)\right|^{2}=\left|\frac{\sin \left[k s_{1}\left(\frac{x}{2 L}+\vartheta_{\mathrm{i}}\right)\right]}{k\left(\frac{x}{2 L}+\vartheta_{\mathrm{i}}\right)}\right|^{2} \propto N\left(\vartheta_{\mathrm{i}}\right) .
$$

Therefore the number of atoms impinging on the transmission grating at an angle $\vartheta_{\mathrm{i}}$ is distributed according to $N\left(\vartheta_{\mathrm{i}}\right)$ as given by Eq. (5.52). Fig. 5.25 shows a plot of this distribution which attains a full width at half maximum (FWHM) of $0.012 \mathrm{mrad}$.

The quantum mechanical amplitude on the grating for a definite particle velocity and incident angle, and the width of the region which is reached by a significant number of atoms are the result of the amplitude $A\left(x, \vartheta_{\mathrm{i}}\right)$ from Eq. (5.51) being diffracted by the second collimation slit. If the transmission grating were placed right behind the second collimator the incident amplitude $\Psi_{0}\left(x, \vartheta_{\mathrm{i}}\right)$ on the grating would obviously be equal to $A\left(x, \vartheta_{\mathrm{i}}\right)$, and the width of the illuminated spot would be equal to the width $s_{2}=10 \mu \mathrm{m}$ of the second collimation slit which in view of the period $d=100 \mathrm{~nm}$ of the transmission grating would correspond to a sharply bounded region of $N=100$ illuminated slits. In reality, the transmission grating is placed $150 \mathrm{~mm}$ behind the second collimation slit and the incident quantum mechanical amplitude $\Psi_{0}\left(x, \vartheta_{\mathrm{i}}\right)$ on the grating can be calculated in the Fresnel picture of diffraction, which yields

$$
\Psi_{0}\left(x, \vartheta_{\mathrm{i}}\right) \propto \int_{-\frac{s_{2}}{2}}^{\frac{s_{2}}{2}} \mathrm{~d} x^{\prime} A\left(x^{\prime}, \vartheta_{\mathrm{i}}\right) \mathrm{e}^{\mathrm{i} k \sqrt{\left(x-x^{\prime}\right)^{2}+Z^{2}}}
$$

where $Z=150 \mathrm{~mm}$ is the distance from the second collimator at $z=-150 \mathrm{~mm}$ to the grating at $z=0$. The result of a numerical evaluation of Eq. (5.53) is displayed in Fig. 5.26 where the intensity $\left|\Psi_{0}(x, 0)\right|^{2}$ and the phase $\arg \left[\Psi_{0}(x, 0)\right]$ of the quantum mechanical amplitude $\Psi_{0}(x, 0)$ are plotted as a function of the lateral coordinate $x$ on the grating, for the case of normal incidence $\vartheta_{\mathrm{i}}=0$. It 


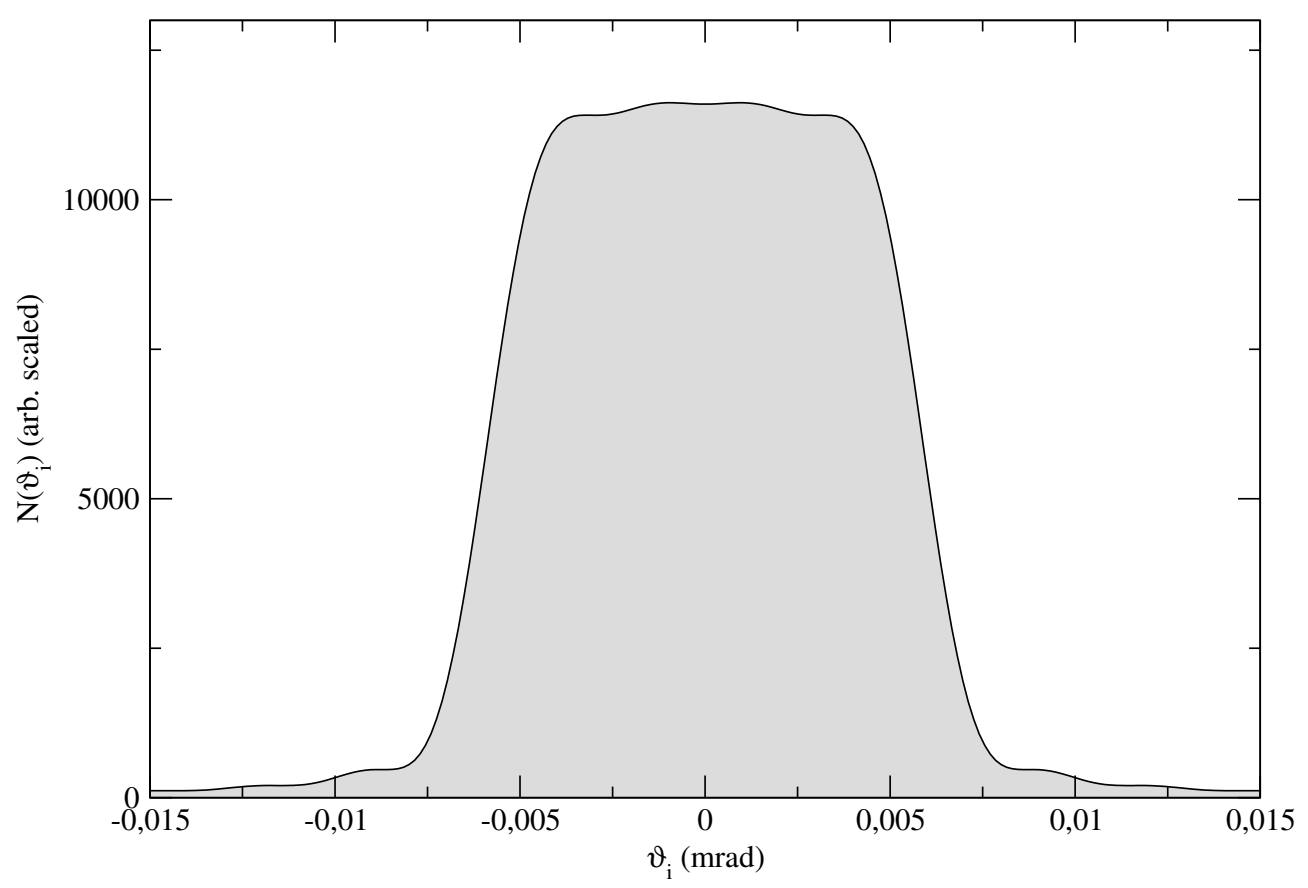

Figure 5.25: The distribution $N\left(\vartheta_{i}\right)$ of incident angles assumes a full width at half maximum of $0.012 \mathrm{mrad}$ as a consequence of the width of the second collimation slit (cf. Fig. 5.24).

can be seen that the number of incident atoms is significantly greater than zero over a region including about $N=80$ grating periods. Within that region the quantum mechanical phase varies by less than $\pi$ so that it is roughly justified to replace each of the monochromatic incident wavefunctions by a plane wave in order to facilitate further calculations as presented in chapter 2. The number of illuminated grating slits and the coherence of the incident wavefunction can be considerably enhanced by narrowing the first collimation slit to $s_{1}=10 \mu \mathrm{m}$, as is illustrated in Fig. 5.27.

Two examples of measured diffraction patterns of metastable $\mathrm{He}^{*}$ and $\mathrm{Ne}^{*}$ are shown in Fig. 5.28 and Fig. 5.29, respectively. The data points for the intensity given in counts per second are the averaged result of 5 minutes $\left(\mathrm{He}^{*}\right)$ and 8 minutes $\left(\mathrm{Ne}^{*}\right)$ measuring time. The velocities $v=2347 \frac{\mathrm{m}}{\mathrm{s}}\left(\mathrm{He}^{*}\right)$ and $v=873 \frac{\mathrm{m}}{\mathrm{s}}$ $\left(\mathrm{Ne}^{*}\right)$ of the atoms follow from an analysis of the principal order peak positions in the diffraction patterns. Each pattern represents the signal of the channel plate detector for a certain time-of-flight window while during the two experiments with $\mathrm{He}^{*}$ and $\mathrm{Ne}^{*}$ many of those windows are measured simultaneously with the help of an appropriate triggering of the detector as explained above. The width of the windows, i.e. the uncertainty in the time-of-flight leads to distribution of incident velocities for each diffraction pattern whose width $\Delta v$ is about $3 \%$ of 


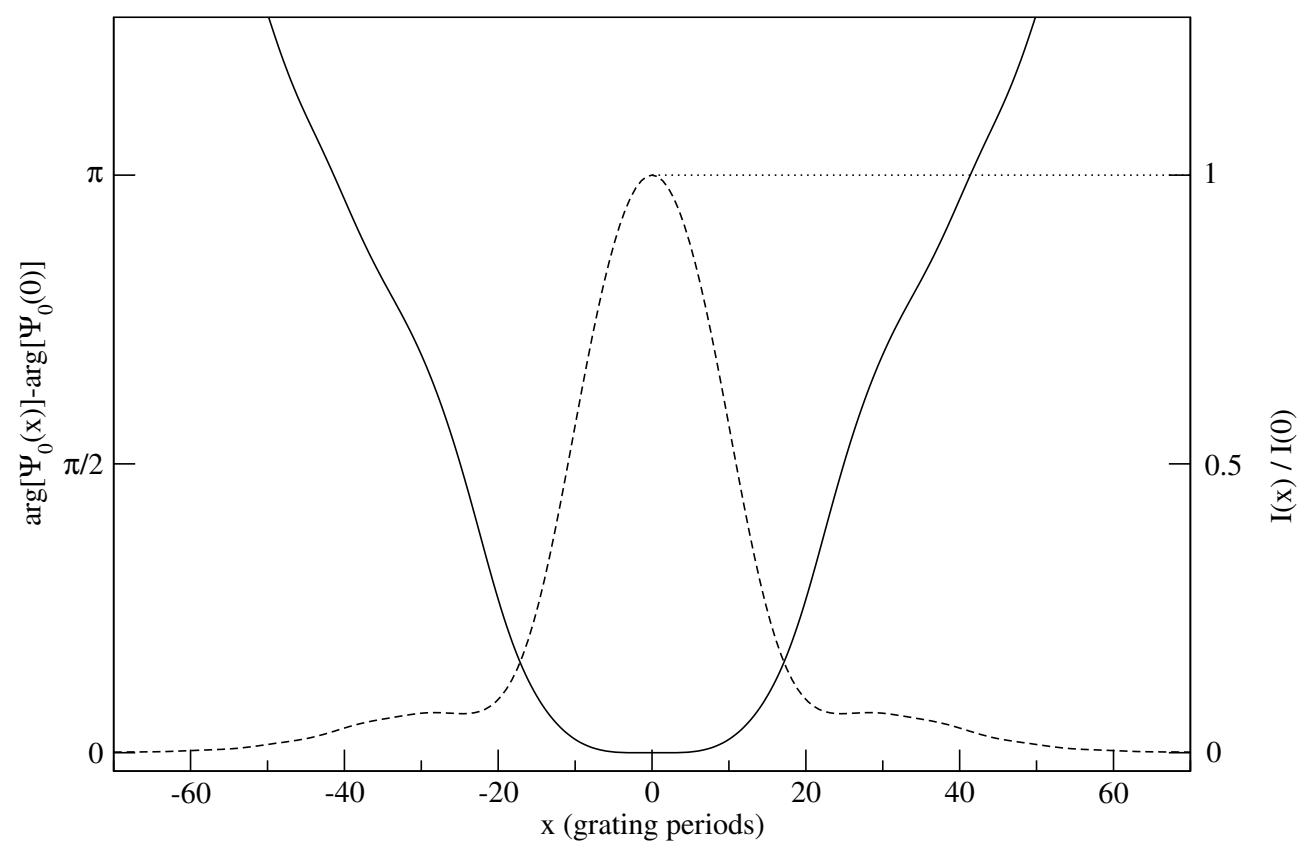

Figure 5.26: As a result of the collimation of a monochromatic wavefunction the incident quantum mechanical amplitude on the transmission grating assumes values as shown in the plot where the dashed line represents the normalized intensity corresponding to the right vertical scale, and the solid line is the phase corresponding to the left vertical scale. It can be seen that for the width of the first and the second collimation slit in the experiment about 80 slits are significantly illuminated while the phase over that region changes by less than $\pi$. One may thus speak of an incident wavefront of ground-state He atoms at $v=1769.9 \frac{\mathrm{m}}{\mathrm{s}}$ which is coherent over 80 grating periods.

the mean velocity $v$. The peak shape results from the width of the detector slit and the distribution of angles and velocities of the beam impinging on the grating. For increasing diffraction orders the peaks become wider because of the velocity distribution. By fitting a sum of Gaussian curves to the diffraction patterns the areas of the peaks are determined. The relative areas have been shown in chapter 1 and chapter 3 to correspond to the theoretical diffraction intensities of the respective diffraction orders. In the case of metastable neon the detector slit is comparatively wide so that the low diffraction orders overlap in the measured pattern. Furthermore, the higher order peaks exhibit somewhat distorted shapes whose analytic areas, though, are close to the areas determined with the Gaussian fit. 


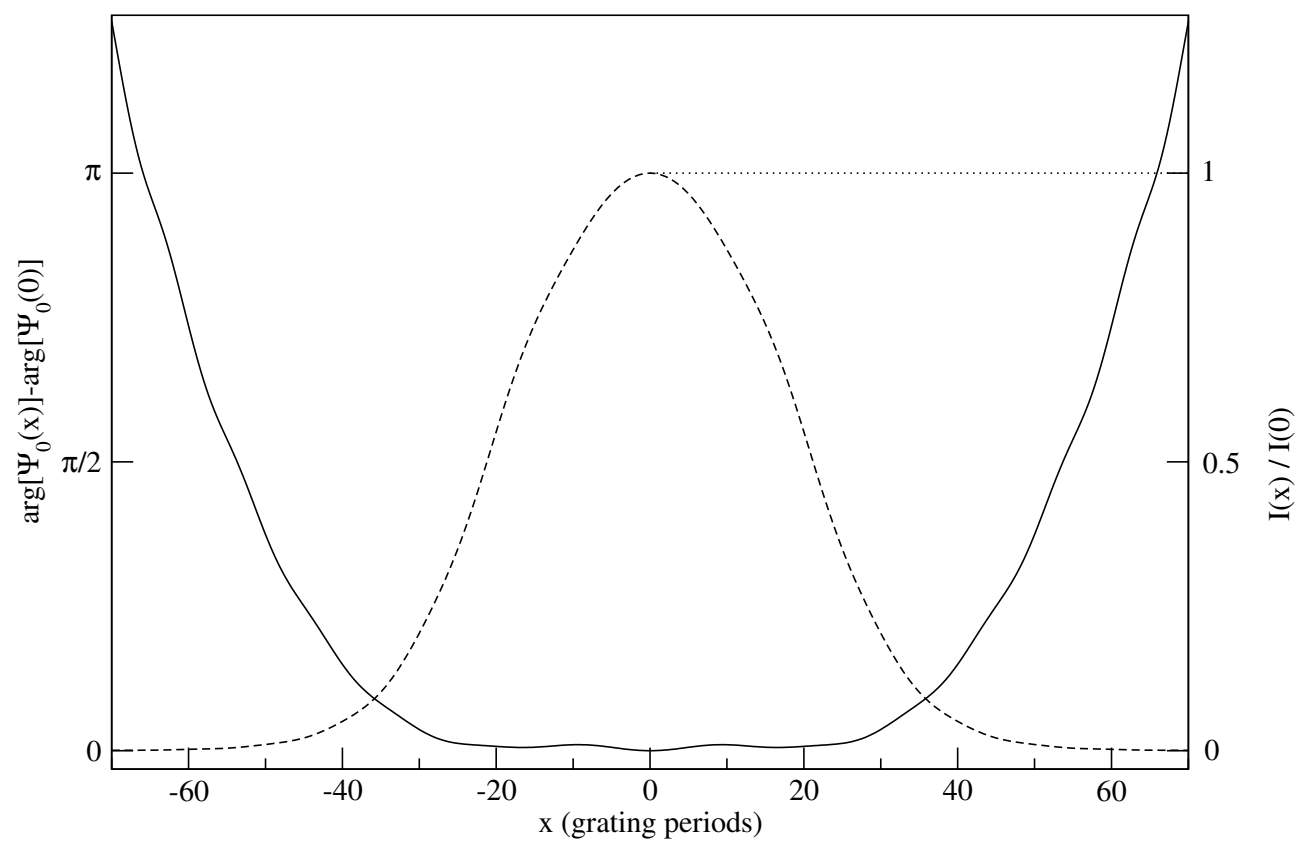

Figure 5.27: This figure is analogous to Fig. 5.26 except for the width of the first collimation slit which is taken half as wide as before. It becomes evident that the modification helps to enhance the width of the coherently illuminated spot on the grating which puts the experiment closer to the theoretical concept of an incident plane wave.

\subsubsection{Error Sources in the Apparatus}

There are a few systematic sources of error in the experiment. During the angular scan it is possible that the absolute intensity of the beam varies with time which would affect the count rates and hence the intensities of the diffraction orders measured at that moment. The intensity at each angle is recorded for about 5-10 minutes. In order to detect such a varying absolute intensity the scan has to be repeated, which has not been done in the $\mathrm{He}^{*}$ and $\mathrm{Ne}^{*}$ experiments because of the limited measuring time available. Another way to check for the beam stability is to measure both positive and negative diffraction angles and compare the symmetrical diffraction orders whose intensities with a stable beam should be equal. Unfortunately, there has been measured only one side of the diffraction pattern. A repeated scan or a scan of both sides is also interesting in that it will show if the above mentioned distorted peak shapes of metastable neon can be reproduced which would make it necessary to extend the theory that to-date cannot predict any peak shape similar to the measured distorted ones.

A comparison of both sides of a diffraction pattern also reveals if the grating normal is oriented parallel to the incident beam which refers especially to an 


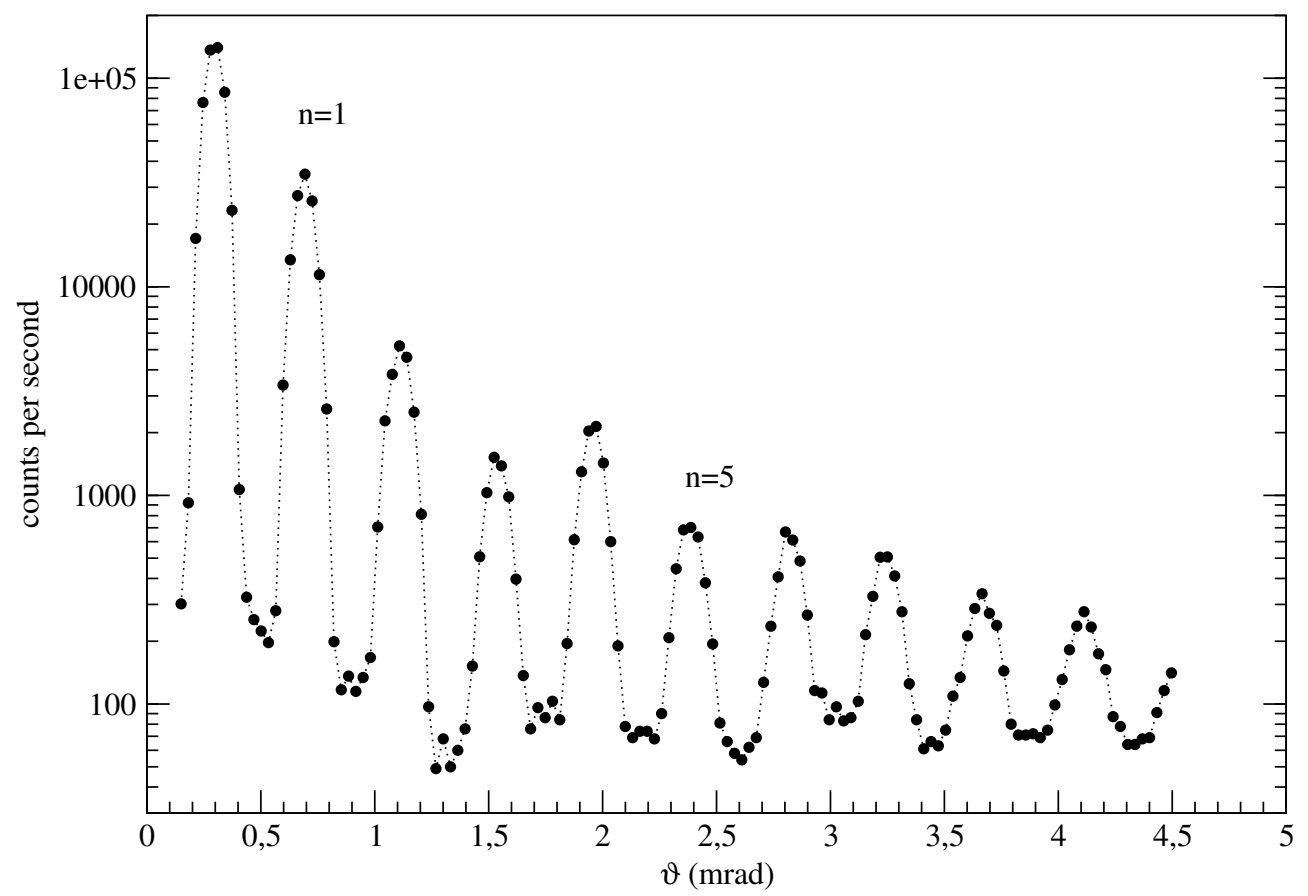

Figure 5.28: Measured diffraction pattern of metastable helium at a velocity $v=$ $2347 \frac{\mathrm{m}}{\mathrm{s}}$. At least 9 principal diffraction orders are shown to be clearly resolved by the experimental technique.

erroneous grating rotation angle around the vertical $y$-axis passing through the centre of the grating. While a forward or backward leaning of the grating has no grave consequences on the measurement a possible rotation of the grating around the normal is expected to lead to small asymmetries in the diffraction patterns. It is assumed that the grating has been possible to be fixed in a position that aligns the bars within a few angular degrees around the vertical, so the rotation of the grating around the grating normal and the forward and backward leaning are thought of as included in the error bars of the result.

For a clockwise rotation $\tau$ of the grating around the vertical axis the theory set out in chapter 4 predicts that the positive low order diffraction intensities are higher than their symmetrical negative counterparts. It has been tested that an erroneous rotation of $\tau=3^{\circ}$ leads to an error of about $20 \%$ in the dispersion interaction strength $C_{3}$ which comes out as a fit parameter when theoretical diffraction intensities are fitted to the corresponding measured data. In the experiment, the rotation angle $\tau$ of the grating has not been specified but has been aimed to be set to zero.

For $\mathrm{He}^{*}$ there exists a preliminary diffraction measurement on grating 5-3-1 without a time-of-flight analysis which is shown in Fig. 5.30. The wide distri- 


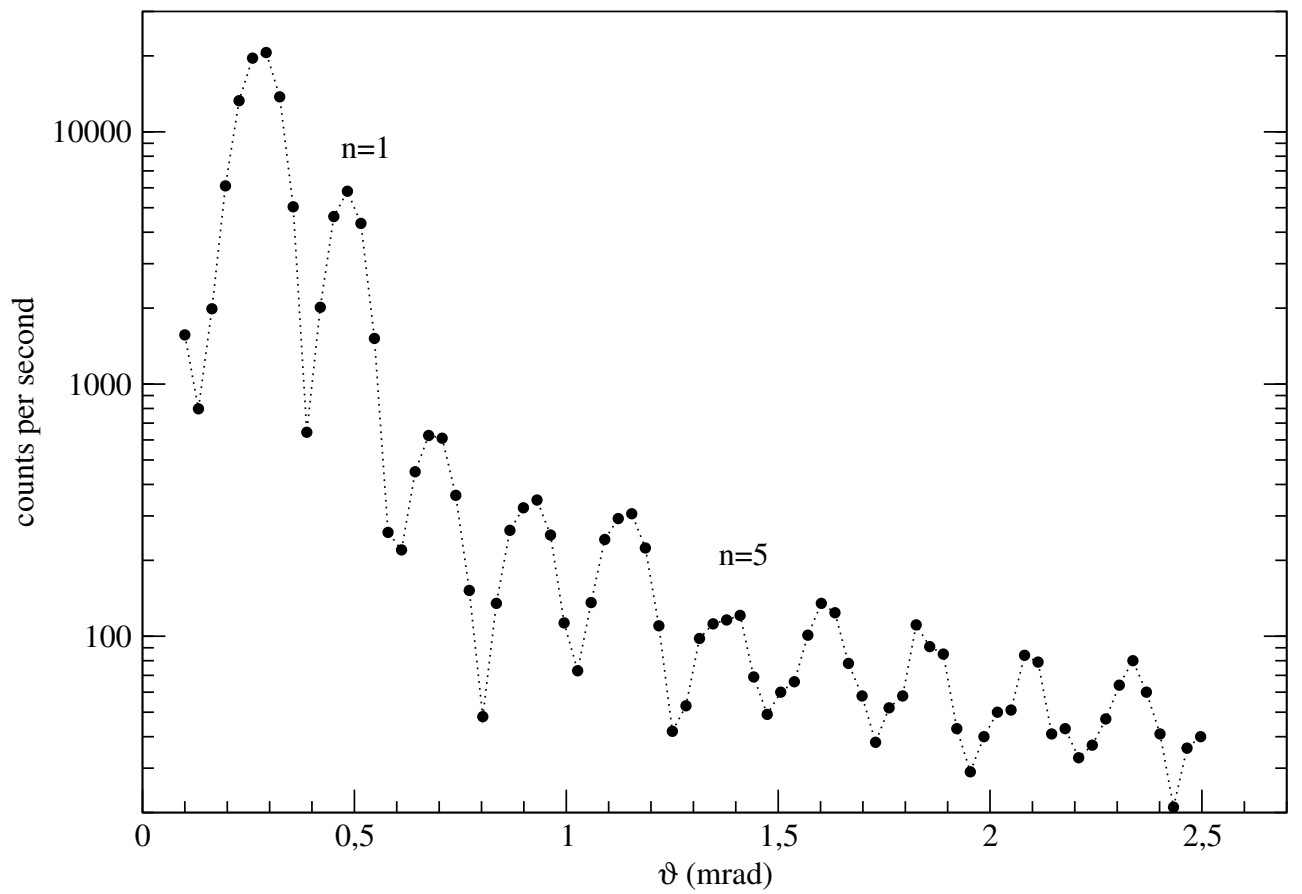

Figure 5.29: Measured diffraction pattern of metastable neon at a velocity $v=873 \frac{\mathrm{m}}{\mathrm{s}}$. The overlap of the low-order peaks is due to the width of the detector slit.

bution of incoming velocities allows only for the \pm first diffraction orders to be clearly seen, where the stress lies on the fact that the first and minus first order can be compared in order to check for an erroneous grating rotation $\tau$. The areas under the -1 st, 0 th, and 1st order peaks are, respectively, in arbitrary units $I_{-1}=1752 \pm 27, I_{0}=6829 \pm 54$, and $I_{+1}=1654 \pm 27$. Excluding the possibility that the absolute beam intensity was higher when the negative angles were scanned and adopting the geometrical parameter set of the grating 5-3-1 as determined earlier the measured intensity ratios $\frac{I_{-1}}{I_{0}}$ and $\frac{I_{+1}}{I_{0}}$ are reproduced to within $0.1 \%$ by theoretical values according to Eq. (4.42) ff. Hereby the grating rotation angle $\tau=(-3.3 \pm 0.5)^{\circ}$ and the dispersion coefficient $C_{3}\left(\mathrm{He}^{*}\right)=(4.25 \pm 0.7) \mathrm{meV} \mathrm{nm}^{3}$ of $\mathrm{He}^{*}$ with $\mathrm{SiN}_{x}$ have been used as fit parameters, where the errors mainly arise from the Gaussian fit that has been used to extract the experimental peak areas. The result for the dispersion coefficient $C_{3}\left(\mathrm{He}^{*}\right)$ is in good agreement with the theoretical value $C_{3}\left(\mathrm{He}^{*}\right)=3.8 \mathrm{meV} \mathrm{nm}^{3}$ calculated in chapter 3 .

It should be stressed that this method of obtaining the dispersion coefficient $C_{3}$ from a two-parameter fit to two experimental peak intensities is more accurate than it may seem. Fig. 5.30 shows that the peaks are distinctly shaped by many data points so that there is small doubt about the experimental peak areas. Theoretically, it has been shown that collimation and Fresnel effects do not affect the 


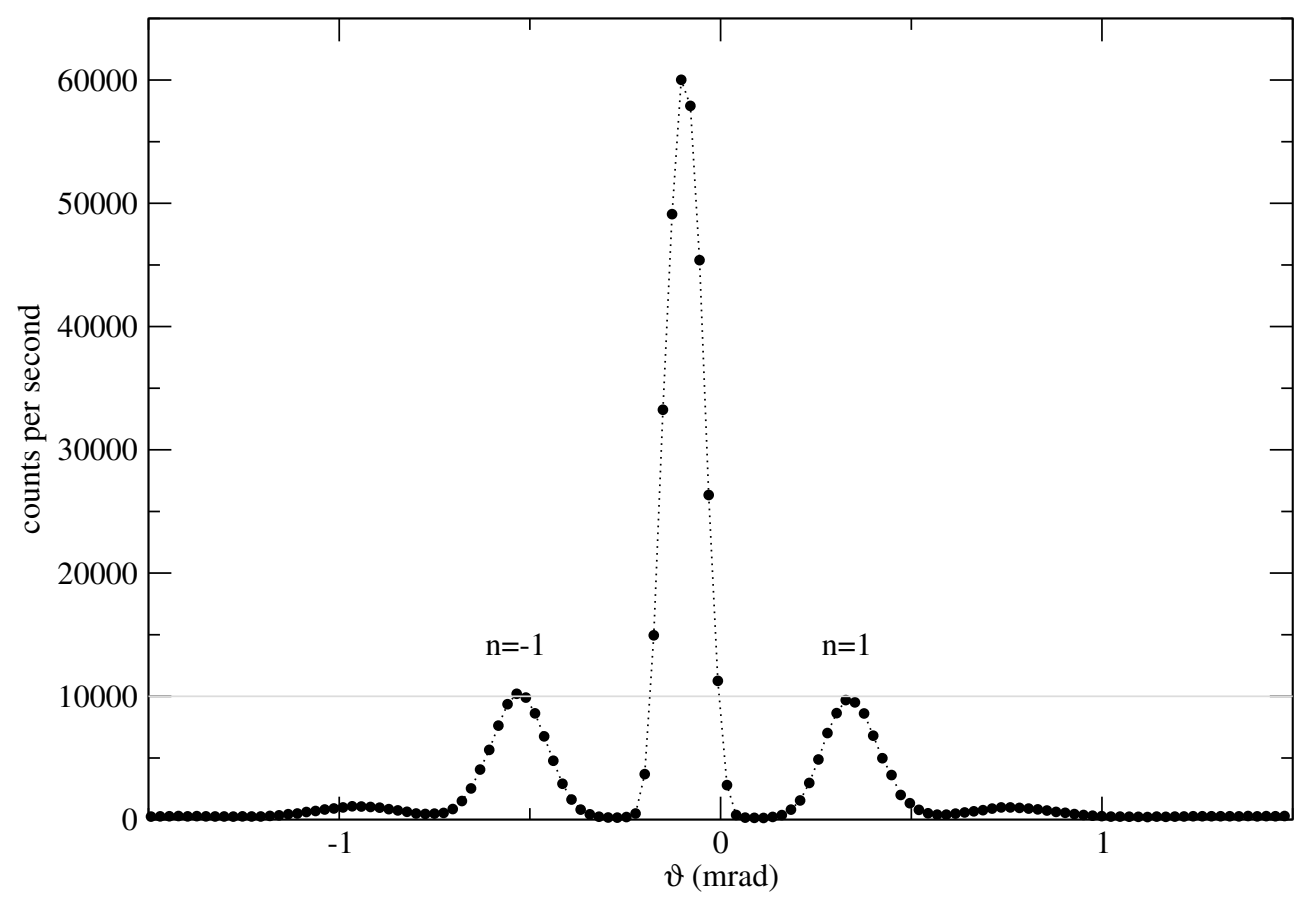

Figure 5.30: Measured diffraction pattern of metastable helium at $v=2285.0 \frac{\mathrm{m}}{\mathrm{s}}$ without the TOF device. The large number of data points per peak allows the areas of the -1 st, 0 th, and 1st maximum to be accurately determined. From the asymmetry of the -1 st and 1st intensity (the left peak is slightly bigger than that on the right) and the ratio to the 0th order intensity an erroneous grating rotation and the dispersion interaction strength $C_{3}$ of $\mathrm{He}^{*}$ can be determined.

concept that the intensity ratios based on the Fraunhofer picture are practically equivalent to the measured relative peak areas. Small errors in the slit width $s$ are included in the stated errors for the dispersion strength $C_{3}$; as for the wedge angle $\beta$ it has been pointed out with the help of an example of $\mathrm{He}$ and $\mathrm{D}_{2}$ earlier on that for large dispersion interaction strengths $C_{3}$ like those of the metastable atoms the particles effectively interact with the grating bars over the whole depth $t$ so that they tend to be sensitive to the average of the local inclinations of the bar side which is given by the total wedge angle $\beta=(11 \pm 0.5)^{\circ}$, which in view of the new improved technique seems to be a reliably measured quantity. The influence of the bar edge roughness has been shown to be small for diffraction orders with high intensities among which the above used \pm 1 st orders belong.

What also makes this simple approach towards finding the dispersion coefficient $C_{3}$ favourable as compared to the available time-of-flight analyzed scans over a large range of angles is that due to its heaviness the detector arm of the apparatus does not run smoothly on the rail which leads in a circle around the location of the grating at the origin of the use coordinate frame. Occasionally, 
the driving force of the motor is obstructed by strains in the bending parts that connect the grating chamber with the detector chamber, or when the wheels at the bottom side of the detector unit encounter an uneven spot on the rail, or when the rail is pushed out of level by the weight of the detector so that a downhill force bends and twists the apparatus and causes it to lean to one side. While the numerical values of the angle which are recorded reflect only the revolutions of the angular drive the detector slit happens to remain obstructed at certain positions, or to follow the drive in a jerk at others. This leads to errors in the centre positions of the diffraction peaks, in their width, and distortions of the peak shapes similar to those observed for $\mathrm{Ne}^{*}$ in Fig. 5.29. The areas of certain peaks in this case are subject to observed fluctuations of up to $30 \%$. What also enters at this point is that the stress in the apparatus during the angular scan leads to shear forces which actually displace the detector slit with respect to the detector so that the maximum of the diffracted image of the detector slit falls on a region of different sensitivity on the channel plate. This can amount up to a $10 \%$ variation in the detection efficiency which is proportional to the measured count rates.

Given the large number of error sources which have been mostly unrecognized and hence not specified during the measurement the best way is to include in the analysis a possibly large number of diffraction patterns at different velocities because then the errors can to some degree be expected to be averaged out of the fit.

\subsubsection{Dispersion Interaction Strength}

In order to determine the dispersion interaction coefficient $C_{3}$ from the measured diffraction intensities a first approach is the $s_{\text {eff }}\left(\frac{1}{\sqrt{(v)}}\right)$ method as described in section 5.1.3. It has been pointed out in this section that the interpretation of the slope of the approximately linear function $s_{\text {eff }}\left(\frac{1}{\sqrt{(v)}}\right)$ in terms of the dispersion interaction strength $C_{3}$ is hindered if the range of the dispersion potential is small as compared to the depth $t$ of the grating bars because the particles in this case interact with a small portion of the grating bar side which has a local wedge angle $\beta^{\text {loc }}$ different from the experimentally determined total wedge angle $\beta$. As mentioned earlier this restriction is not expected to be relevant for metastable atoms because of their stronger dispersion interaction potential. Therefore it can be tried to apply the $s_{\text {eff }}\left(\frac{1}{\sqrt{(v)}}\right)$ method to the metastable atoms, with the additional advantage that Eq. (5.11) can be used including the zeroth order diffraction intensity as there are known to be no clusters of metastable atoms in the beam. The approximations used to predict that the graph of $s_{\text {eff }}\left(\frac{1}{\sqrt{(v)}}\right)$ be a straight line are no longer rigorously justified for the metastable atoms but with the geomet- 
rical slit width $s$ already available the effective slit width determined from the diffraction intensities of metastable atoms can be used to estimate the dispersion interaction strength $C_{3}$. It is possible to obtain the effective slit width $s_{\text {eff }}$ in this case as the three-parameter fit of Eq. (5.11) to the measured intensities with the independent parameters $s_{\text {eff }}, \delta$, and $\sigma_{0}$ actually works well for diffraction intensity patterns of the metastable atoms. Fig. 5.31 shows two typical examples of measured $\mathrm{He}^{*}$ and $\mathrm{Ne}^{*}$ diffraction intensities (=the area under the respective peaks
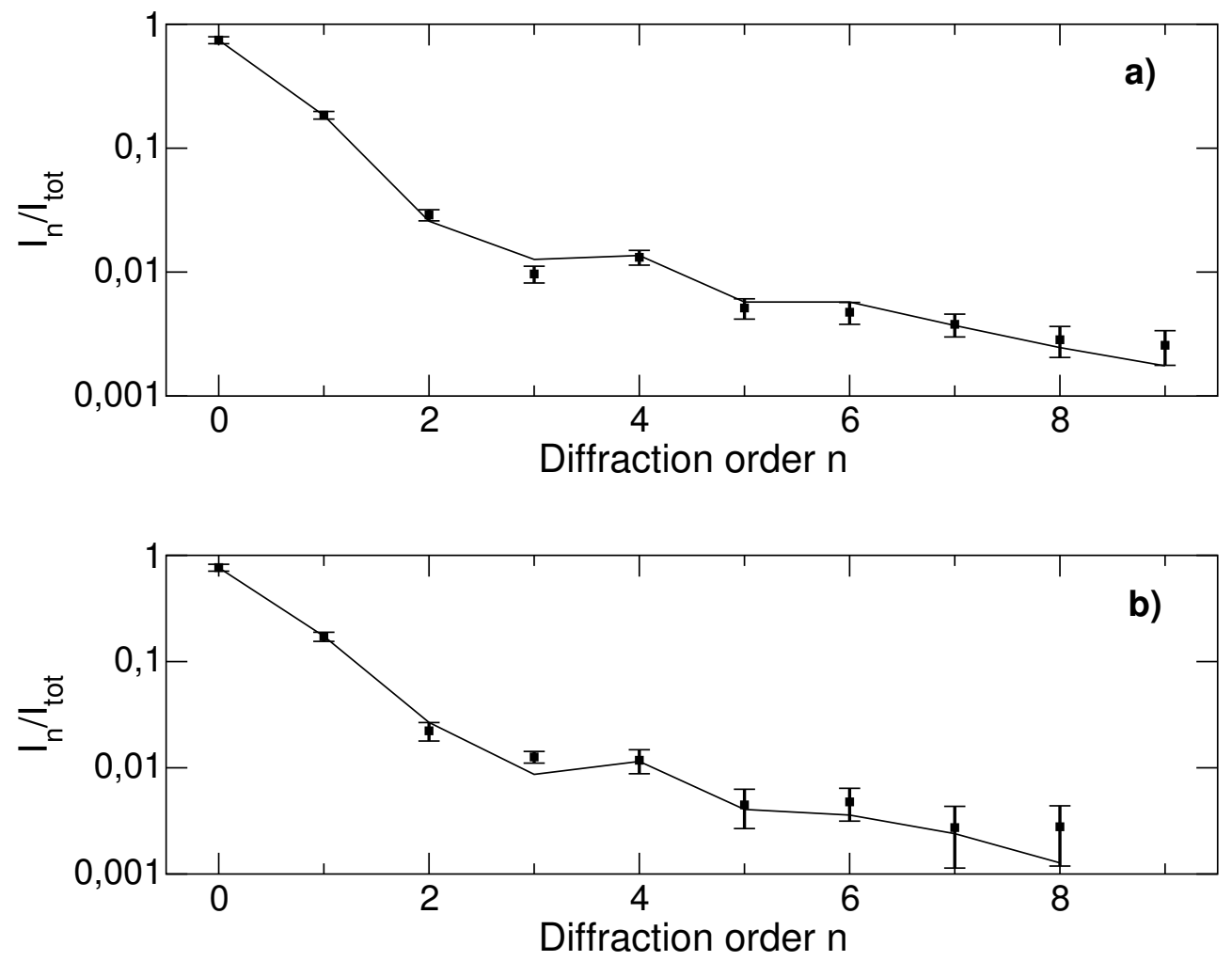

Figure 5.31: With the help of the experimental diffraction intensities of a) $\mathrm{He}^{*}$ at $v=2347 \frac{\mathrm{m}}{\mathrm{s}}$ and $\left.\mathrm{b}\right) \mathrm{Ne}$ at $v=873 \frac{\mathrm{m}}{\mathrm{s}}$ it is shown that the cumulant based formula Eq. (5.11) (solid lines) is suitable to describe the experiment.

in the measured diffraction patterns) and the fitted theoretical curves according to Eq. (5.11).

It remains to show that Eq. (5.11) is a suitable approximation of the theoretical diffraction intensities that are derived from scattering theory. Eq. (5.11) is based on the cumulant expansion Eq. (5.9) and has been discussed in section 5.1.3. There it is shown that the expansion can be restricted to the first two terms in order to describe the theoretical diffraction intensities of ground-state He, based on a theoretically expected dispersion potential. With the help of the example of $\mathrm{He}^{*}$ which has the stronger dispersion interaction of the two and is 
thus furthest from the case of ground-state atoms it is investigated if same is true for the metastable species $\mathrm{He}^{*}$ and $\mathrm{Ne}^{*}$. Fig. 5.32 illustrates the result of the comparison between the exact theory and the cumulant-based Eq. (5.11) for ground-state $\mathrm{He}$ and metastable $\mathrm{He}^{*}$ atoms at $v=2000 \frac{\mathrm{m}}{\mathrm{s}}$ diffracted from a virtual grating 5-3-1. It can be seen that by using only the first two terms in the
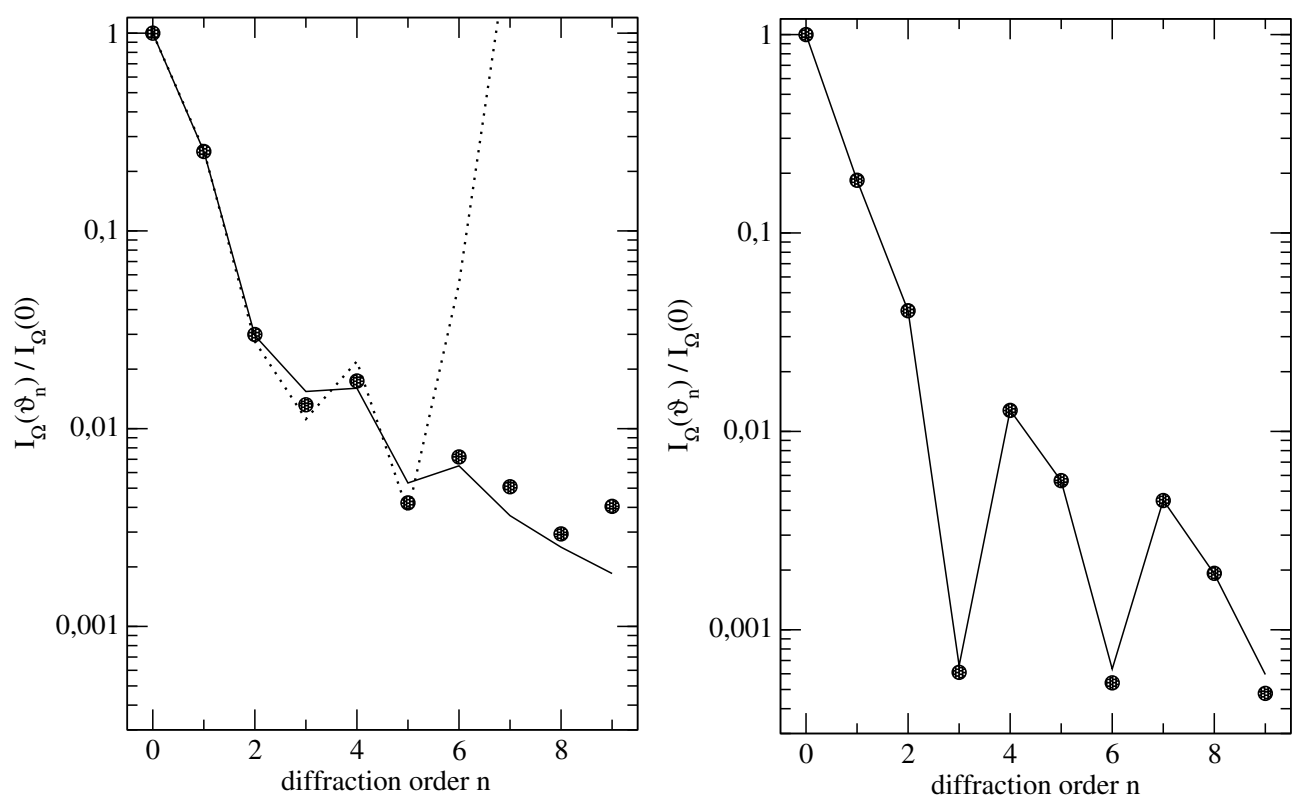

Figure 5.32: The left panel shows that the theoretical diffraction intensities of metastable $\mathrm{He}^{*}$ at $v=2000 \frac{\mathrm{m}}{\mathrm{s}}$ (full circles) are in reasonable agreement with the kumulant approximation Eq. (5.11) (solid line) whose parameters $s_{\text {eff }}, \delta$, and $\sigma$ are calculated according to Eq. (5.12) ff. By including two more cumulants in the approximation (cf. Eq. (5.56) ) the situation is only improved up to the fifth diffraction order (dotted line). For orders greater than 5 the approximation breaks down quickly because the cumulant expansion Eq. (5.9) converges slowly. For ground-state helium at the same beam velocity (right panel, full circles) the two-cumulant approximation Eq. (5.11) (right panel, solid line) works well because the dispersion interaction strength of He is much smaller than that of $\mathrm{He}^{*}$.

cumulant expansion good convergence is achieved for ground-state He atoms up to the eighth diffraction order (Fig. 5.8 is just re-plotted here for a comparison) whereas for metastable $\mathrm{He}^{*}$ atoms already the third diffraction order displays a slight mismatch which, however, does not deteriorate much with increasing diffraction order. The reason for the worse convergence in the latter case lies in the fact that the dispersion interaction of $\mathrm{He}^{*}$ with $\mathrm{SiN}_{x}$ is 50 times stronger than that of ground-state He.

If two more terms are included in the cumulant expansion Eq. (5.9), with the 
appropriate next two cumulant coefficients

$$
R_{3}=-R_{1}^{3}-3 R_{1} R_{2}+\left(\frac{s}{2}\right)^{3}-3 \int_{0}^{\frac{s}{2}} \mathrm{~d} \zeta \zeta^{2} \tau(\zeta)
$$

and

$$
R_{4}=-R_{1}^{4}-6 R_{1}^{2} R_{2}-3 R_{2}^{2}-4 R_{1} R_{3}+\left(\frac{s}{2}\right)^{4}-4 \int_{0}^{\frac{s}{2}} \mathrm{~d} \zeta \zeta^{3} \tau(\zeta)
$$

the equivalent of Eq. (5.11) becomes

$$
\frac{I_{n}}{I_{0}}=\frac{\mathrm{e}^{-\left(\frac{2 \pi n \tilde{\sigma}(n)}{d}\right)^{2}}}{\left(\frac{\pi n}{d}\right)^{2}\left(\tilde{s}_{\mathrm{eff}}^{2}(n)+\tilde{\delta}^{2}(n)\right)}\left[\sin ^{2}\left(\frac{\pi n \tilde{s}_{\mathrm{eff}}(n)}{d}\right)+\sinh ^{2}\left(\frac{\pi n \tilde{\delta}(n)}{d}\right)\right] .
$$

The essential difference of this Eq. (5.56) and the former Eq. (5.11) is that the parameters $\tilde{s}_{\text {eff }}(n), \tilde{\delta}(n), \tilde{\sigma}(n)$ now attain a dependence on the diffraction order $n$. They are given by

$$
\begin{array}{r}
\tilde{s}_{\mathrm{eff}}(n):=s_{\mathrm{eff}}+\Delta s_{\mathrm{eff}}(n) \equiv s-2 \operatorname{Re}\left(R_{1}\right)+\frac{1}{6}\left(\frac{\pi n}{d}\right)^{2} \operatorname{Re}\left(R_{3}\right), \\
\tilde{\delta}(n):=\delta-\Delta \delta(n) \equiv 2 \operatorname{Im}\left(R_{1}\right)-\frac{1}{6}\left(\frac{\pi n}{d}\right)^{2} \operatorname{Im}\left(R_{3}\right),
\end{array}
$$

and

$$
\tilde{\sigma}^{2}(n):=\sigma^{2}-\Delta \sigma(n) \equiv \sigma_{0}^{2}+\operatorname{Re}\left(R_{2}\right)-\frac{1}{12}\left(\frac{\pi n}{d}\right)^{2} \operatorname{Re}\left(R_{4}\right) .
$$

From direct fits of theoretical to experimental diffraction intensities it turns out that the higher diffraction order maxima for metastable atoms are subject to an only small Debye-Waller damping, i.e. the damping parameter $\sigma_{0}$ is practically set equal to zero. This is an example of a higher order effect of the corrugation as the lowest order description given earlier predicts the same damping for groundstate and metastable atoms. Because of the strong interaction of the metastable atoms with the bars the interaction potential for a given trajectory at intermediate distances from the surface is averaged over a comparatively long stretch of the bar surface which suppresses the damping effect of the surface corrugation. For smaller distances where the local corrugation becomes important the large interaction strength of the metastable atoms leads to rapid oscillations of the quantum mechanical phase which significantly reduces the influence of this range of distances on the diffraction intensities. 
For ground-state atoms, the correction terms $\Delta s_{\text {eff }}(3)=0.007 \mathrm{~nm}, \Delta \delta(3)=$ $0.007 \mathrm{~nm}, \Delta \sigma(3)=0.004 \mathrm{~nm}^{2}$ in Eq. (5.56) calculated for the third order diffraction intensity $(n=3)$ are small compared to the respective values of $s_{\text {eff }} \approx 65 \mathrm{~nm}$, $\delta \approx 3 \mathrm{~nm}$, and $\sigma^{2} \approx(2.3)^{2} \mathrm{~nm}^{2}$ which explains the good convergence apparent in the Fig.5.32. For metastable $\mathrm{He}^{*}$ atoms, on the other hand, the corresponding numbers are $\Delta s_{\text {eff }}(3)=0.09 \mathrm{~nm}, \Delta \delta(3)=0.19 \mathrm{~nm}, \Delta \sigma(3)=0.11 \mathrm{~nm}^{2}$. Generally, the cumulant expansion is sure to converge because of the denominator $j$ ! under the sum over $j$, but the convergence is significantly slowed down in the case of metastable atoms, as is seen from the large absolute values of the newly included cumulant coefficients $R_{3}$ and $R_{4}$. In particular, for diffraction orders higher than $m=5$ the parameter $\tilde{\sigma}^{2}(n>m)$ becomes negative which leads to an exponential rise of $\frac{I_{n>m}}{I_{0}}$ in Eq. (5.56). This effect is visible in the left panel of Fig. 5.32 where alternatively to the two-cumulant-formula Eq. (5.11) also the four-cumulant formula Eq. (5.56) is plotted.

Apparently, no persistent improvement can be achieved in the cumulant method by including two more coefficients, however, as can be seen from Fig. 5.9 the theoretical accuracy of the two-cumulant formula Eq. (5.11) with respect to the diffraction intensities based on the exact theory is about the same as that in the fit of Eq. (5.11) to measured diffraction intensities as shown in Fig.5.9. As the deviations in both cases arise from different causes, namely the limitations of the cumulant approximation one one side and experimental errors on the other, it is reasonable to assume that by fitting Eq. (5.11) to measured diffraction intensities of metastable atoms there will be some truth in the values of $s_{\text {eff }}\left(\frac{1}{\sqrt{v}}\right)$ that are obtained in the fit.

Besides the result that has been obtained earlier for the dispersion strength $C_{3}\left(\mathrm{He}^{*}\right)$ when investigating the possibility of an erroneously rotated grating the application of the $s_{\text {eff }}\left(\frac{1}{\sqrt{v}}\right)$ method provides a second means of checking the results for $C_{3}\left(\mathrm{He}^{*}\right)$ and $C_{3}\left(\mathrm{Ne}^{*}\right)$ that are to be obtained later on. The progress of the $s_{\text {eff }}\left(\frac{1}{\sqrt{v}}\right)$ method as applied to the metastable atoms diffracted from grating 5-3-1 is on display in Fig. 5.33 .

With the slit width $s=66.8 \mathrm{~nm}$ as determined earlier with the help of groundstate He atoms a fit of

$$
s_{\text {eff }}\left(\frac{1}{\sqrt{v}}\right)=s-K \frac{1}{\sqrt{v}}
$$

(cf. Eq. (5.17)) yields $C_{3}\left(\mathrm{He}^{*}\right)=(3.9 \pm 0.5) \mathrm{meV} \mathrm{nm}^{3}$ and $C_{3}\left(\mathrm{Ne}^{*}\right)=(0.9 \pm 0.5)$ $\mathrm{meV} \mathrm{nm}^{3}$. This is in good agreement with the theoretical value $C_{3}^{\text {theo }}\left(\mathrm{He}^{*}\right)=3.8$ meV nm${ }^{3}$ in the case of $\mathrm{He}^{*}$, but with $C_{3}^{\text {theo }}\left(\mathrm{Ne}^{*}\right)=3.6 \mathrm{meV} \mathrm{nm}^{3}$ in no agreement for the case of $\mathrm{Ne}^{*}$ which is already apparent in the wild scattering of the data points of $s_{\text {eff }}\left(\frac{1}{\sqrt{v}}\right)$ in Fig. 5.33 .

The bad performance of $\mathrm{Ne}^{*}$ reflects the fact already mentioned that especially for $\mathrm{Ne}^{*}$ experimental diffraction intensities, a fit to which produces the 


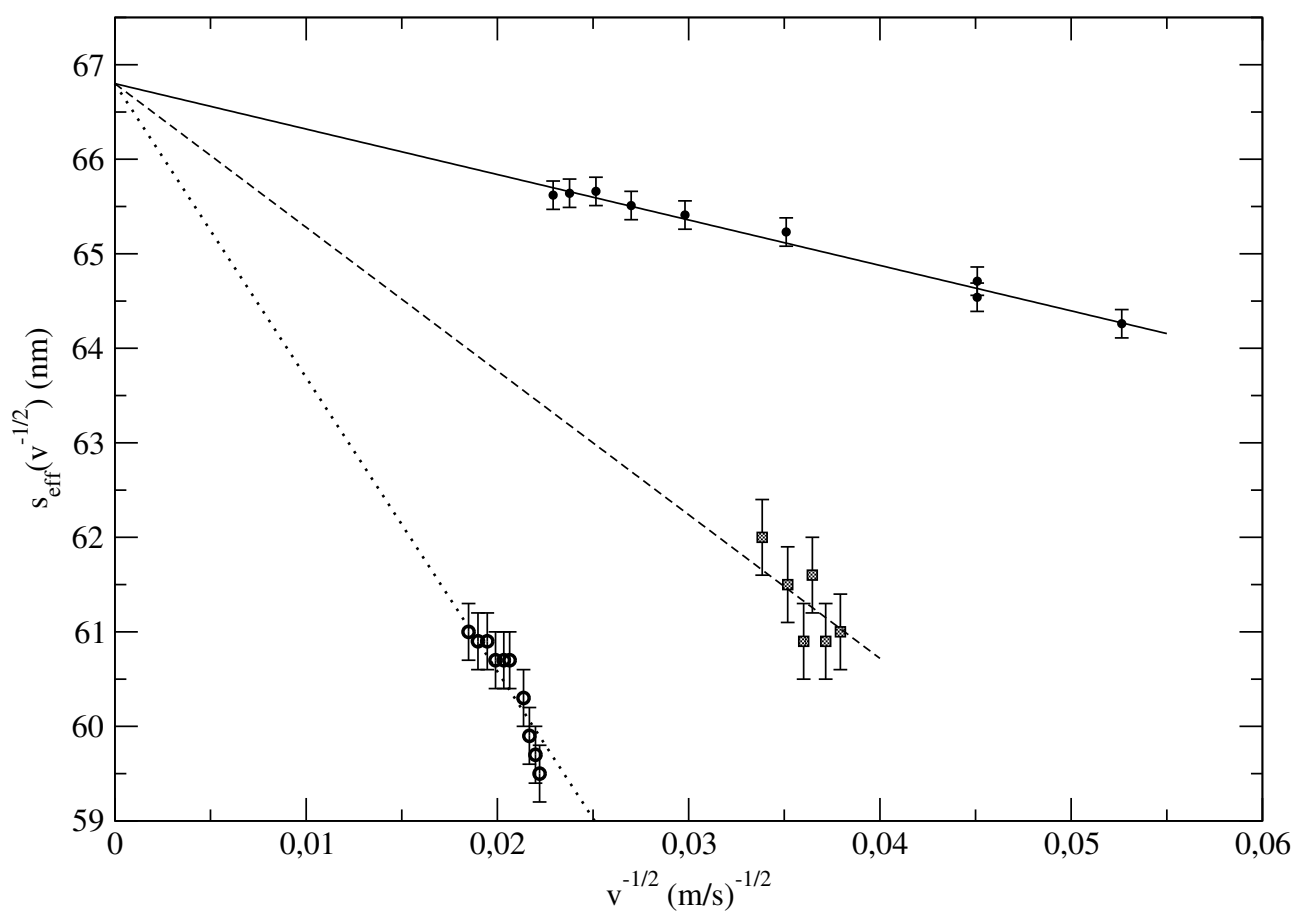

Figure 5.33: The geometrical slit width $s$ determined by extrapolating experimental values of $s_{\text {eff }}\left(\frac{1}{\sqrt{v}}\right)$ for ground-state helium (full circles) is kept fixed as straight lines are fitted to the data points of metastable helium (open circles) and metastable neon (full squares). The slope of the fitted lines is according to Eq. (5.17) used to estimate the dispersion interaction strength $C_{3}$ of the metastable species. The data points of metastable neon display a rather unphysical behaviour which is due to the overlap of the low-order maxima in the measured diffraction patterns.

data points $s_{\text {eff }}\left(\frac{1}{\sqrt{v}}\right)$, are extracted from the experimental diffraction patterns (cf. Fig. 5.29) with errors of up to $30 \%$. The $s_{\text {eff }}\left(\frac{1}{\sqrt{v}}\right)$ method is especially sensitive to the high low-order intensities which in the case of $\mathrm{Ne}^{*}$ coalesce because the detector aperture is too wide as compared to the spacing of subsequent diffraction order peaks while for the lighter $\mathrm{He}^{*}$ atoms the spacing is wider so that subsequent peaks do not coalesce which renders the extraction of the peak areas more accurate. For $\mathrm{He}^{*}$, the newly found value of $C_{3}$ is also in good agreement with that obtained in the analysis of the erroneously rotated grating experiment $C_{3}^{\text {rot }}=(4.2 \pm 0.7) \mathrm{meV} \mathrm{nm}^{3}$, which speaks in favour of the $s_{\text {eff }}\left(\frac{1}{\sqrt{v}}\right)$ method, despite its failure with the diffraction data for metastable $\mathrm{Ne}^{*}$ atoms currently available.

A final method to determine the dispersion interaction strength $C_{3}$ of the metastable $\mathrm{He}^{*}$ and $\mathrm{Ne}^{*}$ atoms is to directly fit theoretical diffraction intensities as given by Eq. (5.7) to experimental intensities extracted as peak areas from 

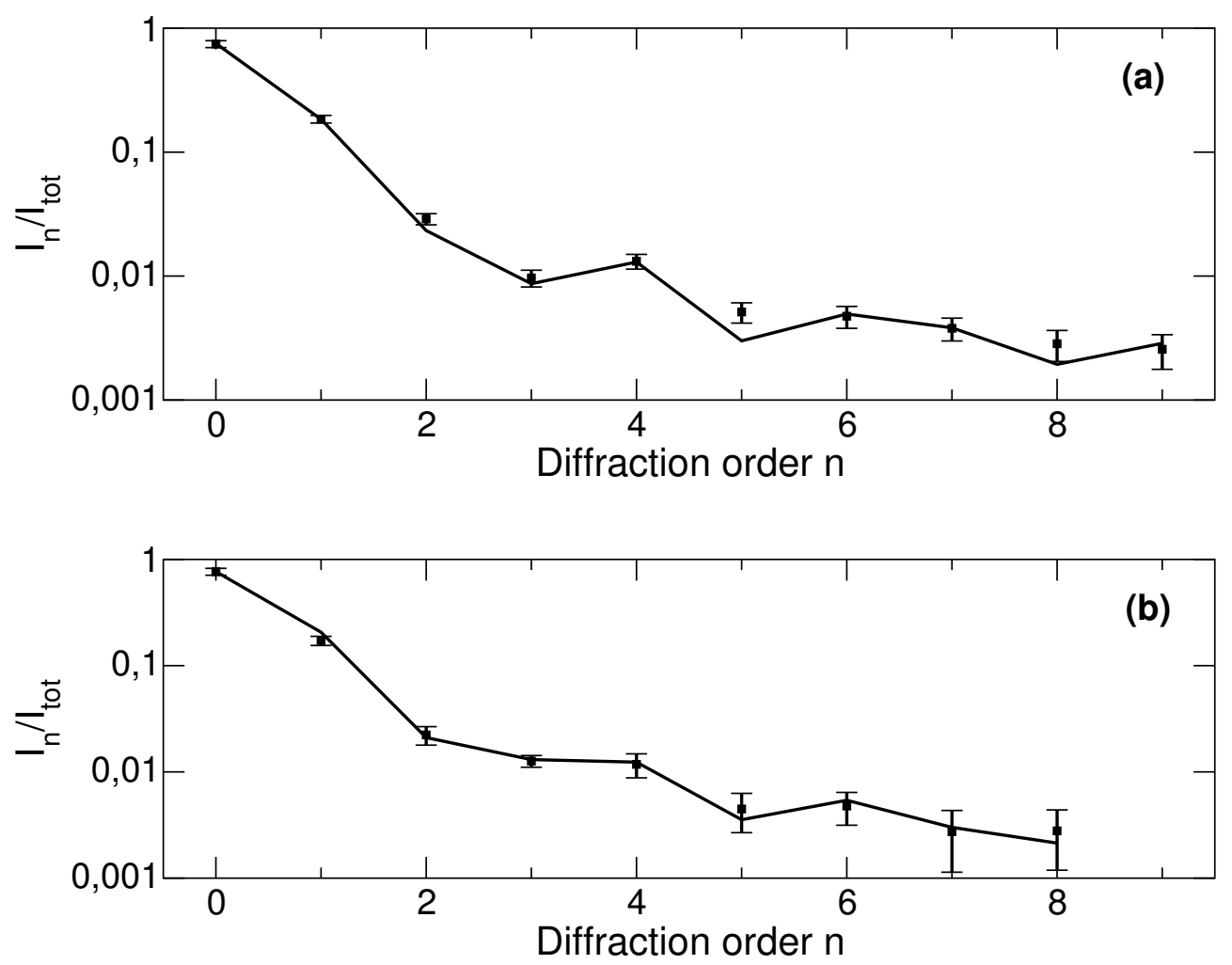

Figure 5.34: Experimental diffraction intensities of a) metastable helium at $v=2347 \frac{\mathrm{m}}{\mathrm{s}}$ and b) metastable neon at $v=873 \frac{\mathrm{m}}{\mathrm{s}}$ are well fitted by theoretical values (solid lines) whereby the dispersion interaction strength $C_{3}$ serves as the only fit parameter.

a number of velocity selected experimental diffraction patterns. For He* 10 sample diffraction patterns have been taken evenly distributed over a range of flight times which corresponds to a range of velocities from $2028 \frac{\mathrm{m}}{\mathrm{s}}<v<2924 \frac{\mathrm{m}}{\mathrm{s}}$, while for $\mathrm{Ne}^{*} 6$ samples have been included in the analysis. The latter have been chosen from a range of low velocities $696 \frac{\mathrm{m}}{\mathrm{s}}<v<873 \frac{\mathrm{m}}{\mathrm{s}}$ because they exhibit a wider spacing between adjacent diffraction orders which minimizes the unwanted overlap of neighbouring peaks. The first diffraction order is left out of the fit as the area of the peak is significantly off the trend for the analyzed diffraction patterns. In particular, the peaks are known from theory to increase in width for increasing diffraction orders. The determined width of the first order peaks is persistently found to be between 10 and $25 \%$ lower than the interpolation between those of the zeroth and the other maxima.

Fitting Eq. (5.7) separately for each velocity to the measured diffraction intensities with the dispersion coefficient $C_{3}$ as only fit parameter one obtains a set of values for $C_{3}(v)$. The final results for $C_{3}$ of $\mathrm{He}^{*}$ and $\mathrm{Ne}^{*}$ are calculated as the weighted average over all $C_{3}(v)$ for the given atomic species, where the weights 
are given by the inverse of the relative mean square deviation $\chi^{2}(v)$ of the fits to the experimental diffraction intensities for the velocities $v \cdot \chi^{2}(v)$ for each velocity $v$ is the average of the squared differences between the theoretical and the experimental value for each diffraction order intensity, divided by the experimental intensity. This standard procedure takes due account of the statistical error $\sqrt{N}$ for a measured count rate of $N$ events per diffraction maximum $t$.

\begin{tabular}{c||c|c} 
method & $C_{3}\left(\mathrm{He}^{*}\right)\left(\mathrm{meV} \mathrm{nm}^{3}\right)$ & $C_{3}\left(\mathrm{Ne}^{*}\right)\left(\mathrm{meV} \mathrm{nm}^{3}\right)$ \\
\hline \hline fit to diffraction intensities & $4.1 \pm 1.0$ & $2.8 \pm 1.0$ \\
$s_{\text {eff }}\left(\frac{1}{\sqrt{v}}\right)$ & $3.9 \pm 0.5$ & $0.9 \pm 0.5^{a}$ \\
rotated grating & $4.25 \pm 0.7$ & - \\
theory & $3.8 \pm 0.1$ & $3.6 \pm 0.1$
\end{tabular}

Table 5.1: Comparison of the values of the dispersion interaction strength $C_{3}$ as determined from the experiment with the theoretical values calculated in chapter 3 .

${ }^{a}$ Systematical errors due to overlapping diffraction maxima.

The quality of the fit of the theory to the measured diffraction intensity is illustrated in Fig.5.34 with the help of two typical examples for $\mathrm{He}^{*}$ and $\mathrm{Ne}^{*}$. The results for the dispersion coefficients $C_{3}$ of $\mathrm{He}^{*}$ and $\mathrm{Ne}^{*}$ are on display in Table 5.1, where they are compared with other measured and theoretical values. It can be seen that for $\mathrm{He}^{*}$ all values of $C_{3}$ determined experimentally by various methods are in good agreement whereas for $\mathrm{Ne}^{*}$ the agreement is less good due to the errors that have been discussed above. For both atomic species the measured values agree with the theoretical values of $C_{3}$ within the error bars.

\subsection{Summary}

The results of this chapter are summarized as follows.

- The dispersion interaction strength $C_{3}$ of metastable $\mathrm{He}^{*}$ and $\mathrm{Ne}^{*}$ atoms with silicon nitride is extracted from measured diffraction intensities by three methods which are all in good agreement with the theoretical values calculated in chapter 3 .

- A detailed account of the error sources is given which have partly been implemented in new experiments and which help at designing future projects.

\footnotetext{
${ }^{4}$ The number of events is actually the sum of the count rates of all data points forming a maximum in the measured pattern times the measuring time. As only relative weights are important in the fit the "number of events" is conveniently normalized to the area of the zeroth order peak.
} 
- The diffraction experiment is for the first time analyzed comprehensively in terms of the beam collimation and Fresnel effects which facilitates current and future experimental work.

- A new method is developed with which the complete atom-surface potential can be measured by atom-diffraction from transmission gratings.

- A systematic misinterpretation of the grating geometry measurements is clarified which explains a $30 \%$ deviation of measured from theoretical values of the dispersion interaction strength $C_{3}$ that has previously been undetected.

- Systematic deviations of experimental from theoretical diffraction intensities whose origin has been unclear so far are now explained as an incoherent effect arising from a variation of the average bar width over the height of the grating. This explanation together with the preceding item of this list leads for the first time to a complete agreement between theoretical and experimental diffraction intensities. 


\section{Chapter 6}

\section{Summary and Outlook}

\subsection{Summary}

The foundations of this work are laid in chapter 2 which gives account of an existing theory of atoms scattered from small structures. As a new feature, the last section is dedicated to the previously neglected fact that in order to describe typical atom diffraction experiments the Fresnel limit has to be applied. It is shown in detail that by appropriately collimating the atomic beam and by choosing a suitable detector aperture the theoretical diffraction intensities are practically equal to those obtained in the Fraunhofer limit of diffraction which is easier to calculate.

The following chapter 3 provides for the first time theoretical values of the dispersion interaction strength $C_{3}$ between ground-state and metastable rare-gas atoms. By reviewing the work of many authors a concise and comprehensive picture is drawn that extends from the quantum electrodynamical roots of the dispersion interaction as part of the van der Waals forces to the description of the dynamic atomic polarizability and goes on to an accurate treatment of the electronic response of dielectric solids, evaluated especially for amorphous silicon nitride which is the transmission grating material used in the experiment. The theory is general in that it includes retardation and it can be applied to all kinds of atom and dielectric solid. With the help of the calculated results a comparison is made with values of $C_{3}$ measured in the past that have since lacked an appropriate theoretical complement.

Starting from the scattering theoretical results of chapter 2 a specific experimental situation is analyzed in chapter 4 . Based on a new technique the atom-grating bar interaction is evaluated in quantitative terms and then generalized to include four new aspects that are of considerable importance for current diffraction experiments and also open the way for new projects in the future. 
First, the diffraction of atoms from rotated gratings is crucial for an accurate determination of the grating bar geometry and also for new experiments whose successful performance depends on a rotation of the transmission grating.

Second, the description of adsorbate layers on the grating bar surface makes it possible to measure the dispersion interaction strength $C_{3}$ for many more atoms and solids. The high accuracy of the atom-optical method can so be applied especially to those materials of interest that cannot be used to make transmission gratings.

As a third generalization of the atom diffraction technique, the influence of retardation is shown to be measurable which is interesting in view of extant theoretical studies on this subject.

The fourth extension of the theoretical concept takes account of the surface corrugation of the bars which is pivotal for a complete understanding of the measured diffraction patterns.

A practical example of the concepts developed in this work is given in chapter 5 which describes an accurate method of determining the dispersion interaction of metastable $\mathrm{He}^{*}$ and $\mathrm{Ne}^{*}$ atoms with the silicon nitride surface. Due to their fragility the atom-optical technique is especially suitable for the metastable atoms and provides unprecedented accuracy. Three methods are presented to extract the values of $C_{3}$ from the measured diffraction patterns which are all in good agreement with the theoretical values from chapter 3 of this work within the error bounds.

To the benefit of present and future diffraction experiments a detailed account of the errors is given as well as a discussion of boundary issues like the beam collimation, the beam composition, and the detector aperture. As a fundamental improvement to the versatility of the current atom diffraction experiments it is shown how the complete atom-surface potential, in particular the depth of the potential well near the surface, can be determined from measured diffraction intensities. For this purpose three experiments are proposed and discussed briefly. The insight into the new concept is greatly enhanced by visualizing the slit amplitude with the help of a parametric plot in the complex number plane.

By analyzing various diffraction experiments it is revealed that previous interpretations of the measured data have suffered from an oversimplification of the grating bar geometry that has led to an error of $30 \%$ in the determined values of $C_{3}$. Along with this the origin of a systematic deviation of measured from theoretical diffraction intensities that has so far been unexplained is clarified and shown to arise from the average grating bar width that varies over the height of the grating. With the latter two improvements the theory developed by our group is now in complete agreement with the measured diffraction intensities. 


\subsection{Outlook}

The detailed analysis of the atom-optical determination of atom-surface potentials as presented in this work is perfectly suitable to be applied in current experimental and theoretical studies done by other groups with metastable argon [14] or large molecules [5, 117]. The metastable atom source is currently re-designed in the MPI group to measure at higher precision the dispersion interaction strength of four metastable species, namely $\mathrm{He}^{*}, \mathrm{Ne}^{*}, \mathrm{Ar}^{*}, \mathrm{Kr}^{*}$. With the help of the given error analysis it will be possible to reduce the error in $C_{3}$ to about $15 \%$ which is suitable to create an experimental standard that can also be extended to other atoms or molecules.

In view of the plans for metastable atom diffraction and other experiments which are aimed at determining the size of helium dimers and trimers from their diffraction intensities it will be advantageous to exploit the possibility of measuring directly the slit width and the bar edge corrugation with scanning electron microscopy. The same insight can be provided by comparing with a certain transmission grating the diffraction intensities of a helium atom beam with those of an X-ray beam that has the same wavelength. As has been pointed out on several occasions, such a comparison is very useful because it is able to clearly separate the effects specific for atoms from general diffraction features.

With experimental data available it is an interesting theoretical topic to calculate the effective change of the atom-bar potential as due to the surface corrugation. Beyond theoretical estimates, there is not much work available in the literature that would quantitatively explore the effect of surface roughness on the potential. As has been shown in this work the simple geometry of the transmission gratings reduces the problem to one dimension as for helium diffraction the corrugation is manifest mainly in the waviness of the trailing bar edges. This constitutes an opportunity for a pioneering quantitative study on the potential between atoms and corrugated surfaces.

As the production of high-quality transmission gratings today is fostered by industrial demand (X-ray gratings for applications in satellites) there is continuous improvement in each new generation of gratings which is very favourable for the on-going diffraction experiments. A particularly fruitful innovation would be to make silicon nitride gratings that are covered with a thin layer of varying materials, so that a standard in the measurement of atom-surface potentials could be established by performing a series of diffraction experiments with a certain type of atom, e.g. ground-state helium, and different gratings each of which is covered with a surface layer of a different material. It has been shown in this work that the diffraction is practically determined by the surface layer material rather that the core material of the grating, so theoretically, the method appears entirely feasible. 
With the experimental equipment available to-date it is possible to determine for the first time the depth of the atom-surface potential well with an atom-optical method as described in this work. The procedure is a consequent generalization of the atom scattering from small structures like the transmission gratings, which is based on the same theoretical foundations as high energy potential scattering. As the latter is nowadays a fully developed branch of physics it is most promising for the future to continue the transfer of know-how to low energy scattering in order to reveal surface properties by atom and molecule diffracted from nanostructured targets. 


\section{Appendix A}

\section{Infinite Half-Space}

Consider an atom at $\mathbf{r}=(x, 0,0)$ opposite an infinite half-space $\left\{\mathbf{r}^{\prime} \in \mathbb{R} \mid x^{\prime}<0\right\}$ whose interaction with each volume element $\mathrm{d}^{3} r^{\prime}$ is given by

$$
W_{6}(\mathbf{r}):=\frac{-K_{6}}{\left|\mathbf{r}-\mathbf{r}^{\prime}\right|^{6}} .
$$

Without restricting generality $\mathbf{r}$ is chosen to be $\mathbf{r}=(l, 0,0)^{\mathrm{T}}$. Then the interaction potential becomes

$$
\begin{aligned}
V_{3}(l) & =\int_{-\infty}^{+\infty} \mathrm{d} y^{\prime} \int_{-\infty}^{+\infty} \mathrm{d} z^{\prime} \int_{-\infty}^{0} \mathrm{~d} x^{\prime} \frac{-K_{6}}{\left[\left(l-x^{\prime}\right)^{2}+\left(y^{\prime}\right)^{2}+\left(z^{\prime}\right)^{2}\right]^{3}} \\
& =-K_{6} \int_{-\infty}^{0} \mathrm{~d} x^{\prime} \int_{-\infty}^{+\infty} \mathrm{d} z^{\prime} \frac{1}{\sqrt{\left(l-x^{\prime}\right)^{2}+\left(z^{\prime}\right)^{2}}} \int_{-\infty}^{+\infty} \mathrm{d} \xi \frac{1}{\left(1+\xi^{2}\right)^{3}} \\
& =\frac{-3 \pi K_{6}}{8} \int_{-\infty}^{0} \mathrm{~d} x^{\prime} \frac{1}{\left(l-x^{\prime}\right)^{4}} \int_{-\infty}^{+\infty} \mathrm{d} \xi \frac{1}{{\sqrt{1+\xi^{2}}}^{5}} \\
& =\frac{-\pi K_{6}}{2} \int_{-\infty}^{0} \mathrm{~d} x^{\prime} \frac{1}{\left(l-x^{\prime}\right)^{4}} \\
& =\frac{-\pi K_{6}}{6 l^{3}} .
\end{aligned}
$$

The atom-surface interaction strength $C_{3}$ can be identified with

$$
C_{3}:=\frac{\pi K_{6}}{6} .
$$

It is possible to derive an atom-surface potential of the general form

$$
V_{i}(l)= \pm \frac{C_{i}}{l^{i}} \quad, \quad i \in \mathbb{N}, i>2
$$


from a similar integration over volume elements of the half-space where for each $i$ a potential

$$
W_{i+3}(\mathbf{r})=\frac{ \pm K_{i+3}}{\left|\mathbf{r}-\mathbf{r}^{\prime}\right|^{i+3}}
$$

is integrated. Hereby the atom-surface interaction strengths $C_{i}$ have to be identified with

$$
C_{i}=\pi K_{i+3}\left[\sum_{j=1}^{i} j\right]^{-1},
$$

which yields $C_{4}=\frac{\pi K_{7}}{10}, C_{5}=\frac{\pi K_{8}}{15}, C_{6}=\frac{\pi K_{9}}{21}$, etc... 


\section{Appendix B}

\section{Grating Bar Potential}

The bar has a trapezoid shape with a base length $b$, a depth $t$, and a wedge angle $\beta$ (see Fig. 4.1 in the text). The van der Waals phase shift $\varphi(x)=\varphi_{\mathrm{r}}(x)+\varphi_{\mathrm{l}}(x)$ arising from an attractive $V_{3}$ potential (cf. Eq. (A.2)) is the sum of contributions from the right and the left bar to the sides of each slit, whose width is denoted by $s$. The coordinate $x$ runs along the rear side of the grating, perpendicular to the bars so that $\zeta:=\frac{s}{2}-x$ is the least distance between a given beam trajectory and the protruding edge at the rear end of the grating bar to the right of the slit. The contribution from the right bar is

$$
\begin{aligned}
\varphi_{\mathrm{r}}(x)=\frac{K_{6}}{\hbar v} \int_{-\infty}^{+\infty} \mathrm{d} z \int_{-t}^{0} \mathrm{~d} z^{\prime} \int_{-\infty}^{+\infty} \mathrm{d} y^{\prime} \int_{\frac{s}{2}-z^{\prime} \tan \beta}^{\frac{s}{2}+b+z^{\prime} \tan \beta} \mathrm{d} x\left[\left(x-x^{\prime}\right)^{2}+\left(y^{\prime}\right)^{2}+\left(z-z^{\prime}\right)^{2}\right]^{-3} \\
=\frac{\pi K_{6}}{2 \hbar v} \int_{-t}^{0} \mathrm{~d} z^{\prime} \int_{\frac{s}{2}-z^{\prime} \tan \beta}^{\frac{s}{2}+b+z^{\prime} \tan \beta} \mathrm{d} x^{\prime} \frac{1}{\left(x-x^{\prime}\right)^{4}} \\
=\frac{C_{3}}{\hbar v} \int_{-t}^{0} \mathrm{~d} z^{\prime}\left\{\frac{1}{\left(-\zeta-b-z^{\prime} \tan \beta\right)^{3}}-\frac{1}{\left(-\zeta+z^{\prime} \tan \beta\right)^{3}}\right\},
\end{aligned}
$$

where in the last step the definition Eq. (A.7) of $C_{3}$ has been used. On further evaluation this yields

$$
\begin{aligned}
& \varphi_{\mathrm{r}}(x)=\frac{C_{3}}{2 \hbar v \tan \beta}\left\{\frac{1}{(\zeta+b)^{2}}+\frac{1}{\zeta^{2}}-\frac{1}{(\zeta+b-t \tan \beta)^{2}}-\frac{1}{(\zeta+t \tan \beta)^{2}}\right\} \\
& =\frac{C_{3}}{2 \hbar v \tan \beta}\left\{\frac{1}{(\zeta+b)^{2}}\left(1-\frac{1}{\left(1-\frac{t \tan \beta}{\zeta+b}\right)^{2}}\right)+\frac{1}{\zeta^{2}}\left(1-\frac{1}{\left(1+\frac{t \tan \beta}{\zeta}\right)^{2}}\right)\right\}
\end{aligned}
$$




$$
\begin{gathered}
=\frac{C_{3}}{2 \hbar v \tan \beta}\left\{\frac{1}{(\zeta+b)^{2}}\left(\frac{\left(1-\frac{t \tan \beta}{\zeta+b}\right)^{2}-1}{\left(1-\frac{t \tan \beta}{\zeta+b}\right)^{2}}\right)+\frac{1}{\zeta^{2}}\left(\frac{\left(1+\frac{t \tan \beta}{\zeta}\right)^{2}-1}{\left(1+\frac{t \tan \beta}{\zeta}\right)^{2}}\right)\right\} \\
=\frac{t C_{3}}{\hbar v \zeta^{3}}\left\{\frac{1+\frac{t \tan \beta}{2 \zeta}}{\left(1+\frac{t \tan \beta}{\zeta}\right)^{2}}-\frac{1}{\left(1+\frac{b}{\zeta}\right)} \frac{1+\frac{t \tan \beta}{2 \zeta\left(1+\frac{b}{\zeta}\right)}}{\left(1+\frac{t \tan \beta}{\zeta\left(1+\frac{b}{\zeta}\right)}\right)^{2}}\right\} .
\end{gathered}
$$

The contribution from the left bar is obtained by replacing in this result the impact parameter $\zeta$ with $s-\zeta$.

According to the generalization stated in Eq. (A.8) ff it is here also possible to derive a result for potentials of other integer power laws. The integrals are of a similar quality and can be found - like those above - in integration tables (e.g. [ए]] ). It is most convenient to express the general result in a way similar to Eq. (B.4). The there following simplifications Eq. (B.5) etc. can in principle be applied to the general result but they lead to a large obscuring number of additional terms and are therefore omitted. One obtains for bar potentials of the form Eq. A.8)

$$
\varphi_{\mathrm{r}}(x)=\frac{C_{i}}{j \hbar v \tan \beta}\left\{\frac{1}{(\zeta+b)^{j}}+\frac{1}{\zeta^{j}}-\frac{1}{(\zeta+b-t \tan \beta)^{j}}-\frac{1}{(\zeta+t \tan \beta)^{j}}\right\},
$$

with $j \equiv i-1$, while the contribution from the left bar is again obtained by replacing $\zeta \mapsto s-\zeta$. 


\section{Appendix C}

\section{Retarded Bar Potential}

In chapter 4 it is shown that due to the retarded atom-bar interaction the quantum mechanical amplitude in the right half of a symmetrical grating slit attains a phase given by Eq. (4.86) which reads

$$
\varphi(x)=\frac{1}{\hbar v \sin \beta} \int_{l_{\mathrm{f}}(x)}^{l_{\mathrm{i}}(x)} \mathrm{d} l\left(\frac{C_{3}(0)}{l^{3}}+\frac{A_{0}}{l^{2}}+\frac{A_{1}}{l}\right) \mathrm{e}^{-A_{2} l} .
$$

The integral is solved by repeatedly integrating by parts the terms in the bracket until one has

$$
\begin{aligned}
\varphi(x)=\frac{1}{\hbar v \sin \beta}\{ & {\left[\mathrm{e}^{-A_{2} l}\left(-\frac{C_{3}(0)}{2 l^{2}}-\frac{A_{0}}{l}+\frac{C_{3}(0) A_{2}}{2 l}\right)\right]_{l_{\mathrm{f}}(x)}^{l_{\mathrm{i}}(x)}+} \\
& \left.+\left(A_{1}-A_{0} A_{2}+\frac{C_{3}(0) A_{2}^{2}}{2}\right) \int_{l_{\mathrm{f}}(x)}^{l_{\mathrm{i}}(x)} \mathrm{d} l \frac{\mathrm{e}^{-A_{2} l}}{l}\right\},
\end{aligned}
$$

where the remaining integral over $l$ gives

$$
\int \mathrm{d} t \frac{\mathrm{e}^{a t}}{t}=\ln t+\sum_{n=1}^{\infty} \frac{(a t)^{n}}{n n !} .
$$

Herewith, and after re-substituting for

$$
l_{\mathrm{f}}(x) \equiv \frac{\zeta}{\cos \beta}
$$

and

$$
l_{\mathrm{i}}(x) \equiv \frac{\zeta+t \tan \beta}{\cos \beta}
$$


one finds

$$
\begin{aligned}
\varphi(x)= & \frac{1}{\hbar v \sin \beta}\left\{\mathrm{e}^{-A_{2} \frac{\zeta+t \tan \beta}{\cos \beta}}\left[-\frac{C_{3}(0) \cos ^{2} \beta}{2(\zeta+t \tan \beta)^{2}}+\frac{\left(C_{3}(0) A_{2}-2 A_{0}\right) \cos \beta}{2(\zeta+t \tan \beta)}\right]-\right. \\
& \left.-\mathrm{e}^{-A_{2} \frac{\zeta}{\cos \beta}}\left[-\frac{C_{3}(0) \cos ^{2} \beta}{2 \zeta^{2}}+\frac{\left(C_{3}(0) A_{2}-2 A_{0}\right) \cos \beta}{2 \zeta}\right]\right\}+ \\
+ & \frac{K_{1}}{\hbar v \sin \beta}\left\{\ln \left(\frac{\zeta+t \tan \beta}{\zeta}\right)+\sum_{n=1}^{\infty}\left(\frac{-A_{2}}{\cos \beta}\right)^{n} \frac{(\zeta+t \tan \beta)^{2}-\zeta^{2}}{n n !}\right\},
\end{aligned}
$$

where the abbreviation

$$
K_{1}:=A_{1}-A_{0} A_{2}+\frac{C_{3}(0) A_{2}^{2}}{2}
$$

has been introduced. The result Eq. (4.83) quoted in the text is obtained upon extraction of a factor $\frac{\cos \beta}{2}$ from the first curly bracket in Eq. (C.6). 


\section{Bibliography}

[1] B. J. Feder, International Herald Tribune, August 20, 2002.

[2] J. Ferrera, M. L. Schattenburg, H. I. Smith Analysis of distortion in interferometric lithography J. Vac. Sci. Technol. B 14,6 4009 (1996); T. A. Savas, M. L. Schattenburg, J. M. Carter, H. I. Smith Large-area achromatic interferometric lithography for $100 \mathrm{~nm}$ period gratings and grids J. Vac. Sci. Technol. B 14,6 4167 (1996); T. A. Savas, S. N. Shah, M. L. Schattenburg, J. M. Carter, H. I. Smith Achromatic interferometric lithography for 100nm-period gratings and grids J. Vac. Sci. Technol. B 13,6 2732 (1995).

[3] P. R. Berman (ed.) Atom interferometry Academic Press, 1997.

[4] C. S. Adams, M. Sigel, J. Mlynek Atom Optics Physics Reports 240 143-210 (1994).

[5] B. Brezger, L. Hackermüller, S. Uttenthaler, J. Petschinka, M. Arndt, A. Zeilinger Matter- Wave Interferometer for Large Molecules Phys. Rev. Lett. 88,10 100404 (2002).

[6] O. Carnal, A. Faulstich, J. Mlynek Diffraction of metastable helium atoms by a transmission grating Appl. Phys. B 53 88-91 (1991).

[7] W. Schöllkopf, J. P. Toennies, T. A. Savas, H. I. Smith A cluster size nanofilter with variable openings between 2 and $50 \mathrm{~nm}$ J. Chem. Phys. 109,219252 (1998).

[8] R. B. Doak, R. E. Grisenti, S. Rehbein, G. Schmahl, J. P. Toennies, C. Wöll Towards realization of an atomic de Broglie microscope: Helium atom focusing using Fresnel zone plates Phys. Lett. Lett 83,21 4229 (1999).

[9] R. E. Grisenti, W. Schöllkopf, J. P. Toennies, G. C. Hegerfeldt, T. Köhler, M. Stoll Determination of the Bond Length and Binding Energy of the Helium Dimer by Diffraction from a Transmission Grating Phys. Rev. Lett. 85,112284 (2000). 
[10] G. C. Hegerfeldt, T. Köhler Deviations from classical optics in matter diffraction and determination of the size of weakly bound molecules Phys. Rev. A 61023606 (2000).

[11] G. C. Hegerfeldt, T. Köhler How to study the elusive Efimov state of the ${ }^{4} \mathrm{He}_{3}$ Molecule through a new atom-optical state-selection technique Phys. Rev. Lett. 84,15 3215 (2000).

[12] R. E. Grisenti, W. Schöllkopf, J. P. Toennies, G. C. Hegerfeldt, T. Köhler Determination of atom-surface van der Waals potentials from transmission grating diffraction intensities Phys. Rev. Lett. 83,9 1755 (1999).

[13] D. Raskin, P. Kusch Interaction between a Neutral Atomic or Molecular Beam and a Conducting Surface Phys. Rev. 179,3 712 (1969).

[14] M. Boustimi, J. Baudon, M. Ducloy, J. Reinhardt, F. Perales, C. Mainos, V. Bocvarski, J. Robert Metastable rare gas atoms scattered by nano- and micro-slit transmission gratings Eur. Phys. J. D 17 141-144 (2001).

[15] H. Failache, S. Saltiel, M. Fichet, D. Bloch, M. Ducloy Resonant van der Waals Repulsion between Excited Cs Atoms and Sapphire Surface Phys. Rev. Lett. 83,26 5467 (1999).

[16] M. Fichet, F. Schuller, D. Bloch, M. Ducloy Van der Waals interactions between excited-state atoms and dispersive dielectric surfaces Phys. Rev. A $\mathbf{5 1 , 2} 1553$ (1995).

[17] F. Shimizu Specular reflection of very slow metastable neon atoms from a solid surface Phys. Rev. Lett 86,6 987 (2001).

[18] F. Shimizu, J. Fujita Giant Quantum Reflection of Neon Atoms from a Ridged Silicon Surface J. Phys. Soc. Jpn. 71,1 5-8 (2002).

[19] F. Shimizu, J. Fujita Reflection type Hologram for Atoms Phys. Rev. Lett. $\mathbf{8 8 , 1 2} 123201$ (2002).

[20] F. Pereira Dos Santos, J. Léonard, J. Wang, C. J. Barrelet, F. Perales, E. Rasel, C. S. Unnikrishnan, M. Leduc, C. Cohen-Tannoudji Bose-Einstein Condensation of Metastable Helium Phys. Rev. Lett. 86,16 3459 (2001).

[21] A. Robert, O. Sirjean, A. Browaeys, J. Poupard, S. Nowak, D. Boiron, C. I. Westbrook, A. Aspect A Bose-Einstein Condensate of Metastable Atoms Science 29246 (2001).

[22] C. Henkel, S. Pötting Coherent transport of matter waves Appl. Phys. B 72 73-80 (2001). 
[23] P. Engels, W. Ertmer, K. Sengstock Magnetic guiding of a slow metastable Ne* beam Opt. Comm. 204 185-194 (2002).

[24] T. Köhler Molekülbeugung an mikrofabrizierten Strukturen Doctoral Thesis, Bericht 19/1998 Max-Planck-Institut für Strömungsforschung, Göttingen, 1998.

[25] G. C. Hegerfeldt, T. Köhler Atomic versus molecular diffraction: influence of breakups and finite size Phys. Rev. A 57,3 2021 (1998).

[26] R. E. Grisenti, G. C. Hegerfeldt, T. Köhler, J. P. Toennies Quantentheorie der Materiewellenbeugung Physikalische Blätter 56,11 53-55 (2000).

[27] J. R. Taylor Scattering Theory Wiley, New York, 1972.

[28] C. J. Joachain Quantum Collision Theory North Holland, Amsterdam, 1975 .

[29] M. L. Goldberger, K. M. Watson Collision Theory John Wiley \& Sons, New York, 1964.

[30] F. W. Byron,Jr, C. J. Joachain, E. H. Mund Potential Scattering in the Eikonal Approximation Phys. Rev. D 8,8 2622 (1973).

[31] L. I. Schiff Approximation Method for High-Energy Potential Scattering Phys. Rev. 103,2 443 (1956).

[32] A. Sommerfeld Vorlesungen über theoretische Physik Band IV Optik Dieterich'sche Verlagsbuchhandlung, Wiesbaden, 1950.

[33] A. Rubinowicz Die Beugungswelle in der Kirchhoffschen Theorie der Beugung 2nd edition, Springer, 1966.

[34] D. Colton, R. Kress Integral Equation Methods in Scattering Theory Wiley, New York, 1983.

[35] W. Magnus, F. Oberhettinger, R. P. Soni Formulas and Theorems for the Special Functions of Mathematical Physics 3rd edition, Springer, 1966.

[36] L. W. Bruch, M. W. Cole, E. Zaremba Physical Adsorption: Forces and Phenomena Clarendon, Oxford, 1997.

[37] H. Hoinkes The physical interaction potential of gas atoms with singlecrystal surfaces, determined from gas-surface diffraction experiments Rev. Mod. Phys. 52933 (1980).

[38] R. Eisenschitz, F. London Über das Verhältnis der van der Waalsschen Kräfte zu den homöopolaren Bindungskräften Z. Phys. 60491 (1930). 
[39] F. London Zur Theorie und Systematik der Molekularkräfte Z. Phys. 63 245 (1930).

[40] H. B. G. Casimir, D. Polder The Influence Of Retardation on the Londonvan der Waals Forces Phys. Rev. 73,4 360 (1948).

[41] E. M. Lifshitz The theory of molecular attractive forces between solids JETP 2,173 (1956); I. E. Dzyaloshinskii, E. M. Lifshitz, L. P. Pitaevskii The General Theory of van der Waals Forces Advances in Physics 10,39 165 (1961).

[42] C. Cohen-Tannoudji, B. Diu, F. Laloë Quantum Mechanics Volume II, 2nd ed. Wiley, 1977.

[43] A. Shih, D. Raskin, P. Kusch Investigation of the interaction potential between a neutral molecule and a conducting surface Phys. Rev. A 9,2 652 (1974).

[44] A. Shih van der Waals forces between a Cs atom or a CsCl molecule and metal or dielectric surfaces Phys. Rev. A 9,4 1507 (1974).

[45] A. Shih, V. A. Parsegian Van der Waals forces between heavy alkali atoms and gold surfaces: Comparison of measured and predicted values Phys. Rev. A 12,3 835 (1975).

[46] V. A. Parsegian Formulae for the electrodynamic interaction of pointparticles with a substrate Mol. Phys. 27,6 1503 (1974).

[47] M. J. Mehl, W. L. Schaich Theory of neutral atom scattering at long range from metal surfaces Phys. Rev. A 16,3 921 (1977).

[48] M. J. Mehl, W. L. Schaich The van der Waals interaction between an atom and a solid Surf. Sci. 99 553-569 (1980).

[49] M. J. Mehl, W. L. Schaich Quantum theory of neutral-atom scattering at long range from solid cylinders Phys. Rev. A 21,4 1177 (1980).

[50] A. M. Marvin, F. Toigo Van der Waals interaction between a point particle and a metallic surface I. Theory Phys. Rev. A 25,2 782 (1982).

[51] A. M. Marvin, F. Toigo Van der Waals interaction between a point particle and a metallic surface II. Applications Phys. Rev. A 25,2 803 (1982).

[52] A. Landragin, J.-Y. Courtois, G. Labeyrie, N. Vansteenkiste, C. I. Westbrook, A. Aspect Measurement of the van der Waals Force in an atomic mirror Phys. Rev. Lett. 77,8 1464 (1996). 
[53] G. Vidali, G. Ihm, Hye-Young Kim, M. W. Cole Potentials of physical adsorption Surface Science Reports 12 133-181 (1991).

[54] R. Brühl, P. Fouquet, R. E. Grisenti, J. P. Toennies, G. C. Hegerfeldt, T. Köhler, M. Stoll, C. Walter The van der Waals potential between metastable atoms and solid surfaces: Novel diffraction experiments versus theory Europhys. Lett. 59,3 357-363 (2002).

[55] M. Karimi, G. Vidali Long-range interaction between rare-gas atoms or simple molecules and the surfaces of LiF, CaF 2 , and $B N$ Phys. Rev. B 34,4 2794 (1986).

[56] J. M. Hutson, P. W. Fowler, E. Zaremba Quadrupolar contributions to the atom-surface van der Waals interaction Surf. Sci. 175 L775-L781 (1986).

[57] E. A. Power, T. Tirunamachandran Dispersion interactions between atoms involving electric quadrupole polarizabilities Phys. Rev. A 53,3 1567 (1996).

[58] A. Liebsch Density-functional calculation of quadrupolar contributions to the atom-metal van der Waals interaction Phys. Rev. B 35,17 9030 (1987).

[59] X.-P. Jiang, F. Toigo, M. W. Cole The dispersion force of physical adsorption Surf. Sci. 145 281-293 (1984).

[60] X.-P. Jiang, F. Toigo, M. W. Cole The dispersion force of physical adsorption II. Nonlocal theory Surf. Sci. 148 21-36 (1984).

[61] Gyeong-il Kweon, N. M. Lawandy Dispersion interactions between excited atoms Phys. Rev. A 47,5 4513 (1993).

[62] O. Kenneth, I. Klich, A. Mann, M. Revzen Repulsive Casimir Forces Phys. Rev. Lett. 89033001 (2002).

[63] A. D. McLachlan Van der Waals forces between an atom and a surface Molecular Physics 7381 (1963).

[64] S. Rauber, J. R. Klein, M. W. Cole Substrate-Mediated Dispersion Interaction between Adsorbed Atoms and Molecules Surf. Sci. 123 173-178 (1982).

[65] O. Sinanoğlu, K. S. Pitzer Interactions between Molecules Adsorbed on a Surface J. Chem. Phys. 32,5 1279 (1960).

[66] T.Kihara Intermolecular Forces Wiley, 1978.

[67] L. D. Landau, E. M. Lifshitz Course on Theoretical Physics, Volume 5: Statistical Physics Pergamon Press, London, 1958. 
[68] E. A. Power, S. Zienau On the Radiative Contributions to the Van der Waals Force Nuovo Cimento 6,1 7 (1957).

[69] A. D. McLachlan Retarded dispersion forces between molecules Proc. Roy. Soc. A271 387 (1963).

[70] A. D. McLachlan Three-body dispersion forces Molecular Physics 6423 (1963).

[71] C. Mavroyannis The interaction of neutral molecules with dielectric surfaces Mol. Phys. 6593 (1962).

[72] H. Margenau, N. R. Kestner Theory of Intermolecular Forces 2nd ed., Pergamon Press, Oxford, 1971

[73] C. Hättig, B. A. Hess TDMP2 calculation of dynamic multipole polarizabilities and dispersion coefficients of the noble gases $\mathrm{Ar}, \mathrm{Kr}, \mathrm{Xe}$, and Rn J. Phys. Chem. 100 6243-6248 (1996).

[74] M. O. Bulanin, I. M. Kislyakow Dynamic polarizabilities of rare-gas atoms: Helium, Neon, and Argon Optics and Spectroscopy 86,5 632 (1999).

[75] A. Derevianko, W. R. Johnson, M. S. Safranova, J. F. Babb High-precision calculations of dispersion coefficients, static dipole polarizabilities, and atom-wall interaction constants for alkali-metal atoms Phys. Rev. Lett. 82,18 3589 (1999).

[76] R. M. Glover, F. Weinhold Dynamic polarizabilities of two-electron atoms, with rigorous upper and lower bounds J. Chem. Phys. 65,11 4913 (1976).

[77] D. M. Bishop, J. Pipin Dipole, quadrupole, octupole, and dipole-dipolequadrupole polarizabilities and second hyperpolarizabilities at real and imaginary frequancies for helium in the $2^{3} \mathrm{~S}$ state: Dispersion-energy coefficients for interactions between $\mathrm{He}\left(2^{3} \mathrm{~S}\right)$ and $\mathrm{H}\left(1^{2} \mathrm{~S}\right), \operatorname{He}\left(1^{1} \mathrm{~S}\right), \operatorname{He}\left(2^{3} \mathrm{~S}\right)$, or $\mathrm{H}_{2}\left(\mathrm{X}^{1} \Sigma_{\mathrm{g}}^{+}\right)$International Journal of Quantum Chemistry 47 129-134 (1993).

[78] M. Rérat, M. Caffarel, C. Pouchan Dynamic polarizabilities and van der Waals coefficients of the $2^{1} \mathrm{~S}$ and $2^{3} \mathrm{~S}$ metastable states of helium Phys. Rev. A 48,1 161 (1993).

[79] S. Kotochigova, E. Tiesinga, I. Tupitsyn Nonrelativistic ab initio calculation of the interaction potentials between metastable Ne atoms Phys. Rev. A $6 \mathbf{1}$ $042712(2000)$.

[80] M. R. Doery, E. J. D. Vredenbregt, S. S. Op de Beek, H. C. W. Beijerinck, B. J. Verhaar Limit on suppression of ionization in metastable neon traps due to lang-range anisotropy Phys. Rev. A 58,5 3673 (1998). 
[81] Jiong-Ming Zhu, Bin-Lu Zhu, Zong-Chao Yan Dispersion coefficients for interactions between two metastable helium-like atoms Molecular Physics 98,8 529-534 (2000).

[82] Zong-Chao Yan, J. F. Babb Long-range interaction of metastable helium atoms Phys. Rev. A 58,2 1247 (1998).

[83] A. Derevianko, A. Dalgarno Long-range interactions of two metastable raregas atoms Phys. Rev. A 62062501 (2000).

[84] K. T. Tang Dynamic Polarizabilities and van der Waals Coefficients Phys. Rev. 177,1 105 (1969).

[85] T. Savas: Experimental optical data of $\mathrm{SiN}_{\mathrm{x}}$ as used for gratings. Priv. comm. to J. P. Toennies (2001).

[86] L. Asinovsky, F. Shen, T. Yamaguchi Characterization of the optical properties of PECVD SiN $N_{x}$ films using ellipsometry and reflectometry Thin Solid Films 313-314 198-204 (1998).

[87] G. E. Jellison, F. A. Modine, P. Doshi Spectroscopic ellipsometry characterization of thin-film silicon nitride Thin Solid Films 313-314 193-197 (1998).

[88] R. Nietubyc̀, E. Sobczak, O. S̆ipr, J. Vackár̆, A. S̆imůnek Electronic structure of silicon nitride Journal of Alloys and Compounds 286 148-152 (1999).

[89] M. Klanjs̆ek Gunde, M. Maček Infrared Optical Constants and Dielectric Response Functions of Silicon Nitride and Oxynitride Films Phys. Stat. Sol. 183,2 439-449 (2001).

[90] X. Zhang, K. Ding, A. Yang, D. Shao Processing and Characterisation of PECVD Silicon Nitride Films Advanced Materials for Optics and Electronics 6 147-150 (1996).

[91] F. de Brito Mota, J. F. Justo, A. Fazzio Structural properties of silicon nitride Phys. Rev. B 58,13 8323 (1998).

[92] G. Vidali, M. W. Cole The interaction between an atom and a surface at large separation Surf. Sci. 110 10-18 (1981).

[93] M. Boustimi, B. Viaris de Lesegno, J. Baudon, J. Robert, M. Ducloy Atom symmetry break and metastable level coupling in rare-gas atom-surface van der Waals interaction Phys. Rev. Lett. 86,13 2766 (2001).

[94] A. A. Radzig et al. Reference Data on Atoms, Molecules, and Ions Springer Series in Chemical Physics, 1985. 
[95] P. W. Langhoff, R. G. Gordon, M. Karplus Comparisons of dispersion force bounding methods with applications to anisotropic interactions J. Chem. Phys. 55,5 2126 (1971).

[96] Zong-Chao Yan, J. F. Babb, A. Dalgarno, G. W. F. Drake Variational calculations of dispersion coefficients for interactions among $\mathrm{H}, \mathrm{He}$, and $\mathrm{Li}$ atoms Phys. Rev. A 54,4 2824 (1996).

[97] S. Zollner, E. Apen Optical Constants for Metrology of Hydrogenated Amorphous Silicon-Nitrogen Alloys on Si APS Conference Proceedings 550532 (2001).

[98] T. Savas Priv. comm. to R. Brühl (2002).

[99] R. E. Grisenti, W. Schöllkopf, J. P. Toennies, J. R. Manson, T. A. Savas, H. I. Smith He-atom diffraction from nanostructure transmission gratings: The role of imperfections Phys. Rev. A 61033608 (2000).

[100] T. Savas Priv. comm. to J. P. Toennies (2002).

[101] G. C. Hegerfeldt Priv. comm. (2002).

[102] R. Brühl Priv. comm. (2002).

[103] K. Bosch Elementare Einführung in die Wahrscheinlichkeitsrechnung 5th ed. Vieweg, Braunschweig, 1986.

[104] H. Hotop, A. Niehaus, A. L. Schmeltkopf Reactions of excited atoms and molecules with atoms and molecules Z. Phys. 229 1-13 (1969).

[105] A. G. Borisov, D. Teillet-Billy, J. P. Gauyacq Singlet to triplet conversion in low energy metastable helium-metal surface collisions: Auger deexcitation process Surf. Sci. 325 323-335 (1995).

[106] W. Sesselmann, B. Woratschek, J. Küppers, G. Ertl Interaction of metastable noble-gas atoms with transition-metal surfaces: Resonance ionization and Auger neutralization Phys. Rev. B 35,4 1547 (1987).

[107] P. E. Siska Molecular beam studies of Penning ionization Rev. Mod. Phys. 65,2 337-412 (1993).

[108] V. Sandoghar, C. I. Sukenik, E. A. Hinds, S. Haroche Direct Measurement of the van der Waals Interaction between an Atom and its Images in a Micron-Sized Cavity Phys. Rev. Lett. 68,23 3432 (1992).

[109] C. I. Sukenik, M. G. Boshier, D. Cho, V. Sandoghar, E. A. Hinds Measurement of the Casimir-Polder Force Phys. Rev. Lett. 70,5 560 (1993). 
[110] A. Anderson, S. Haroche, E. A. Hinds, W. Jhe, D. Meschede Measuring the van der Waals forces between a Rydberg atom and a metallic surface Phys. Rev. A 37,9 3594 (1988).

[111] M. Gorlicki, S. Ferron, V. Lorent, M. Ducloy Interferometric approaches to atom-surfaces van der Waals interactions in atomic mirrors Phys. Rev. A 61013603 (1999).

[112] R. Marani, L. Cognet, N. Westbrook, C. I. Westbrook, A. Aspect Using atomic interference to probe atom-surface interactions Phys. Rev. A 61 053402 (2000).

[113] J. A. Brand, J. E. Furst, T. J. Gay, L. D. Schearer Production of a highdensity state-selected metastable neon-beam Rev. Sci. Instrum. 63,1 163 (1992).

[114] P. Fouquet, P. K. Day, and G. Witte Metastable $\left(2^{3} S\right)$ helium atom scattering from NiO(100) and Cu(100)surfaces Surf. Sci. 400140 (1998).

[115] R. E. Grisenti Priv. comm. (2002).

[116] I. N. Bronstein, K. A. Semendjajew Taschenbuch der Mathematik 25th edition, Teubner, Stuttgart, 1991.

[117] P. Facchi, A. Mariano, S. Pascazio Mesoscopic Interference Recent Res. Devel. Physics 3 1-29 (2002). 


\section{ACKNOWLEDGEMENTS}

I would like to express my thanks to Prof. Dr. G. C. Hegerfeldt for carefully supervising this work and for his competent advice on conceptual as well as detailed issues. Thorsten Köhler introduced me to the project in a very patient and thoughtful manner which I appreciate a lot.

The collaboration with the group of Prof. Dr. J.P. Toennies at the MPI was very fruitful and enjoyable. In particular, I am grateful to Mr Toennies who let me use a nice and quiet office room at the MPI and who gave me the opportunity to learn from his professional experience. The experimental data on which this work heavily relies have been measured by Robert Grisenti, Peter Fouquet, Rüdiger Brühl, Oleg Kornilov, and Anton Kalinin. In particular, I am indebted to Rüdiger Brühl whose diligent measurements have proven decisive for some important conclusions drawn in this work. Also to my benefit were the numerous discussions with the MPI people and with my collegue Martin Stoll.

For the funding of my dissertation project the support of the Deutsche Forschungsgemeinschaft and the Institut für theoretische Physik der Universität Göttingen is gratefully acknowledged.

I also thank the members of the commission who have agreed to reviewing this thesis and assisting at my disputation, in particular Prof. Dr. K. Schönhammer as coexaminer, PD. Dr. P. Reinke, Prof. Dr. D. Ronneberger, Prof. Dr. H.-U.Krebs, and Prof. Dr. T. Salditt. 


\section{LEBENSLAUF}

\section{Persönliche Daten}

Name:

Vornamen:

Geburtsdatum:

Geburtsort:

Staatsangehörigkeit:
Walter

Christian Peter

15.08.1969

Neumarkt in der Oberpfalz deutsch

\section{Wissenschaftlicher Werdegang}

1993 - 1995: $\quad$ Studiengang Diplom-Physik, Universität Regensburg, Vordiplom (4 Fachsemester).

1995 - 1996: $\quad$ Einjähriges Physikstudium an der University of Reading, England (2 Urlaubssemester). Trimesterprojekt: Local Vibration Modes of Heavy Atoms in an Alkali Halogenide Matrix

1996 - 1999: $\quad$ Studiengang Diplom-Physik, Universität Regensburg, Diplom (6 Fachsemester). Diplomarbeit: Intrinsischer JosephsonEffekt und Phononen in Hochtemperatur-Supraleitern

11/1999 - 11/2002: Promotion am Institut für theoretische Physik der Universität Göttingen in enger Zusammenarbeit mit einer experimentellen Gruppe am Max-Planck-Institut für Strömungsforschung. Titel der Arbeit: Diffraction of metastable rare-gas atoms from nanostructured transmission gratings

\section{Veröffentlichungen}

C.Preis, C. Helm, K.Schmalzl, C. Walter, J. Keller Microscopic Theory of the Coupling of Intrinsic Josephson Oscillations and Phonons Physica C 341-348 1543-1546 (2000).

R. Brühl, P. Fouquet, R. Grisenti, G. C. Hegerfeldt, T. Köhler, M. Stoll, J.P. Toennies, C. Walter Diffraction of Metastable Atoms from Nanostructured Gratings: New Experiments versus Theory Europhysics Letters 59,3 357 (2002). 